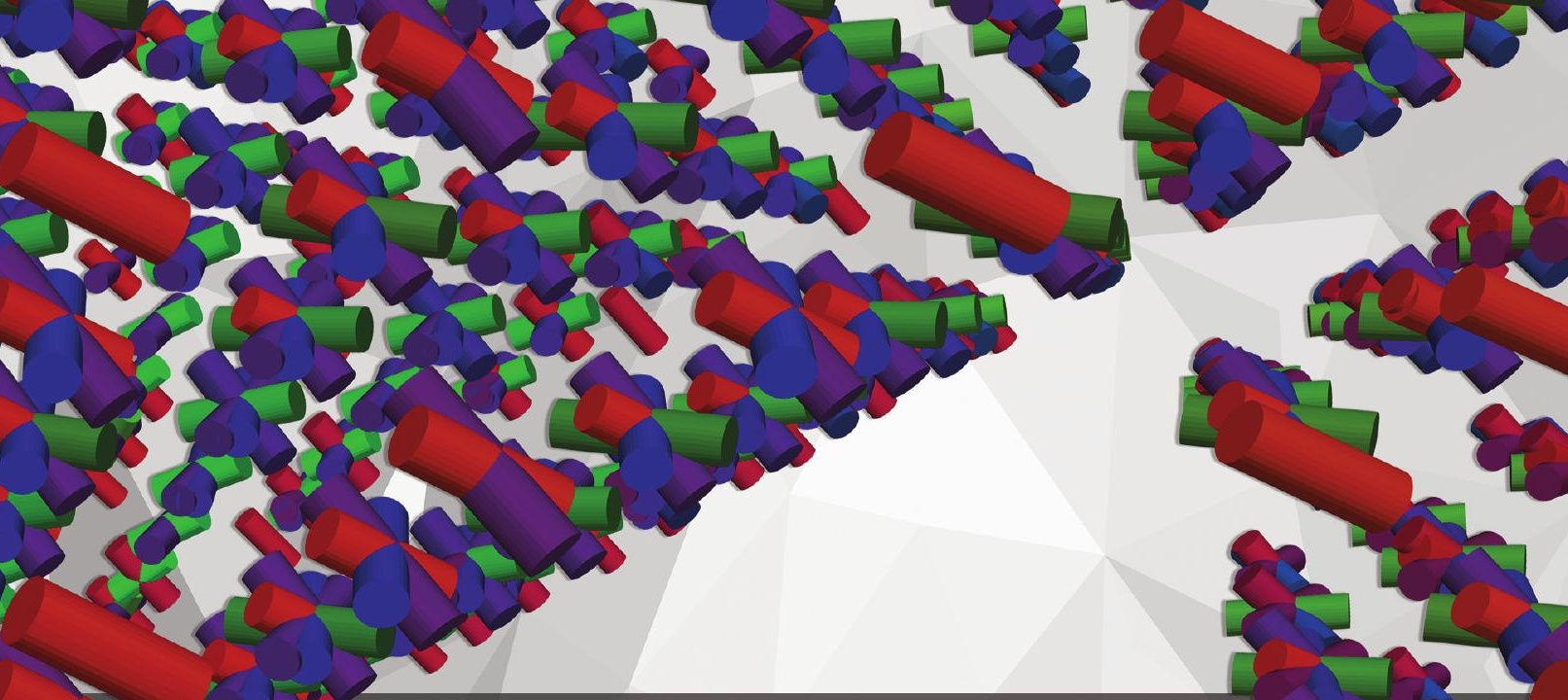

\title{
BIOMECHANICAL MODELS \\ AND PREDICTION OF MOBILITY \\ AFTER TONGUE CANCER SURGERY
}

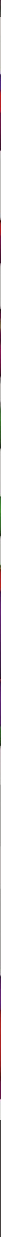




\section{BIOMECHANICAL MODELS AND PREDICTION OF MOBILITY AFTER TONGUE CANCER SURGERY}

Kilian Derk Rinse Kappert 
This dissertation has been approved by:

Supervisors: Dr. ir. F. van der Heijden \& Prof. dr. A.J.M Balm

co-supervisor: Prof. dr. ir. C.H. Slump

Cover design: Kilian Kappert

Printing: Gildeprint Enschede, gildeprint.nl

Lay-out: Anna Bleeker, persoonlijkproefschrift.nl

ISBN: 978-90-365-5098-7

DOI: $10.3990 / 1.9789036550987$

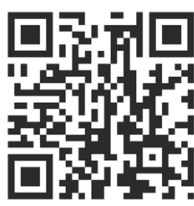

The research conducted in this thesis was made possible by private funding from De Graaf Bakeries BV, Stichting Familie Bert en Alie Verwelius, Mr and Mrs van den Brink - Witte, and Atos Medical AB.

Financial support for publication of this thesis was provided by ChipSoft, the Oncology Graduate School (Netherlands Cancer Institute) and Robotics and Mechatronics (University of Twente)

(c) 2020 Kilian Derk Rinse Kappert, The Netherlands.

All rights reserved. No parts of this thesis may be reproduced, stored in a retrieval systemor transmitted in anyformorby any meanswithout permission of the author. 


\title{
BIOMECHANICAL MODELS AND PREDICTION OF MOBILITY AFTER TONGUE CANCER SURGERY
}

\author{
DISSERTATION
}

to obtain

the degree of doctor at the University of Twente, on the authority of the rector magnificus, prof.dr.ir. A. Veldkamp, on account of the decision of the Doctorate Board to be publicly defended on Friday 18 December 2020 at 14.45

by

\section{Kilian Derk Rinse Kappert}

born on the 12th of July, 1991 in Hengelo, The Netherlands 


\section{Graduation Committee:}

Chairman / secretary:

Prof. dr. J.N. Kok

University of Twente

supervisors:

Dr. ir. F. van der Heijden

University of Twente

Prof. dr. A.J.M Balm

University of Amsterdam

co-supervisor:

Prof. dr. ir. C.H. Slump

University of Twente

Committee Members:

Prof. dr. ir. H.F.J.M. Koopman

University of Twente

Prof. dr. ir. S. Stramigioli

University of Twente

Prof. dr. M.W.M. van den Brekel

University of Amsterdam

Prof. dr. Y. Payan

Université Grenoble Alpes

Dr. M.J.A. van Alphen

Netherlands Cancer Institute 


\section{CONTENTS}

$\begin{array}{lll}\text { Chapter } 1 \quad \text { General introduction } & 8\end{array}$

Chapter 2 An interactive surgical simulation tool to assess the consequences 44 of a partial glossectomy on a biomechanical model of the tongue

Chapter 3 Quantification of tongue mobility impairment using optical 68 tracking in patients after receiving primary surgery or chemoradiation

Chapter 4 Five specific tongue movements in a healthy population 92

Chapter 5 In-vivo Tongue Stiffness Measured By Aspiration: Resting vs 108 General Anesthesia

Chapter 6 Personalized biomechanical tongue models based on diffusion- 140 weighted MRI and validated using optical tracking of range of motion.

Chapter 7 Prediction of tongue function after a partial glossectomy using 162 personalized CSD MRI driven biomechanical models: A pilot study

Chapter 8 Summary and conclusion

Samenvatting en conclusie

$\begin{array}{ll}\text { Appendix A } & 200\end{array}$

$\begin{array}{ll}\text { Appendix B } & 206\end{array}$

$\begin{array}{ll}\text { Appendix C } & 208\end{array}$

$\begin{array}{ll}\text { Acknowledgements / dankwoord } & 212\end{array}$

About the Author 216 
GENERAL INTRODUCTION 
Loss of oral function is one of the key concerns when treating oral and oropharyngeal cancer. Treatment can lead to function losses with unacceptable damage to swallowing, mastication, and speech. But what is unacceptable? In 2011, Kreeft et al. conducted an international global survey among treating physicians to examine whether international consensus exists regarding functional inoperability, resulting in the withholding of treatment due to expected functional loss[1]. This might help to develop guidelines for choosing between surgery or organ-sparing treatment options. The authors discovered considerable variability in defining functional inoperability among head and neck surgeons and radiotherapists worldwide. Half of all surgeons judged a total glossectomy or mandibulectomy to be functionally inoperable, whereas for other procedures there was at most a weak consensus. A high percentage of agreement was only seen in the case of a total glossectomy in combination with a supraglottic laryngectomy, which was judged as functionally inoperable in $85 \%$ of the surveyed physicians.

The findings of this research by Kreeft et al. [1-3] served as the basis for a new research study called 'The Virtual Therapy Project' to objectify expected function loss. This project focuses on the development of virtual models of the oral cavity and oropharynx to predict expected function loss after treatment.

Ten years after the publication of Kreeft et al.'s work, this current thesis constitutes the fourth thesis in a row that deals with the topic of virtual therapy and is built on the research as described in the theses and peer-reviewed publications by Anne Marijn Kreeft, Maarten van Alphen, Merijn Eskes [4-6], and 36 students of whom 8 were graduating students.

This thesis will focus on one of the most important, complex, and fascinating organs in the oral cavity: the tongue. The tongue plays a pivotal role in vital functions like swallowing, mastication, and speech. Advanced tongue cancer in combination with the sequelae after treatment is a disruptive event, and can have a major impact on the quality of life.

This introductory chapter will address several biological aspects of tongue cancer and biomechanical modeling of the tongue. The aim and outline of the thesis are formulated at the end of this chapter. 


\subsection{ANATOMY}

The head and neck region consists of the following five anatomical mucosal subsites: the oral cavity, pharynx (including the nasopharynx, oropharynx, and hypopharynx), larynx, and nasal cavity (Figure 1.1). The major salivary glands, neck, face, and scalp also belong to this anatomical region. The mobile (anterior $2 / 3$ ) tongue is located in the oral cavity and is seamlessly connected to the floor of the mouth. Other structures in the oral cavity include the lips, buccal mucosa, cheek mucosa, gingiva, retromolar trigone, teeth, hard palate, and vallate papillae (Figure 1.2a) [7]. The base (posterior 1/3) of the tongue extends into the oropharynx, which also houses the tonsils, vallecula, the posterior pharyngeal wall, the soft palate, and uvula [3,7].

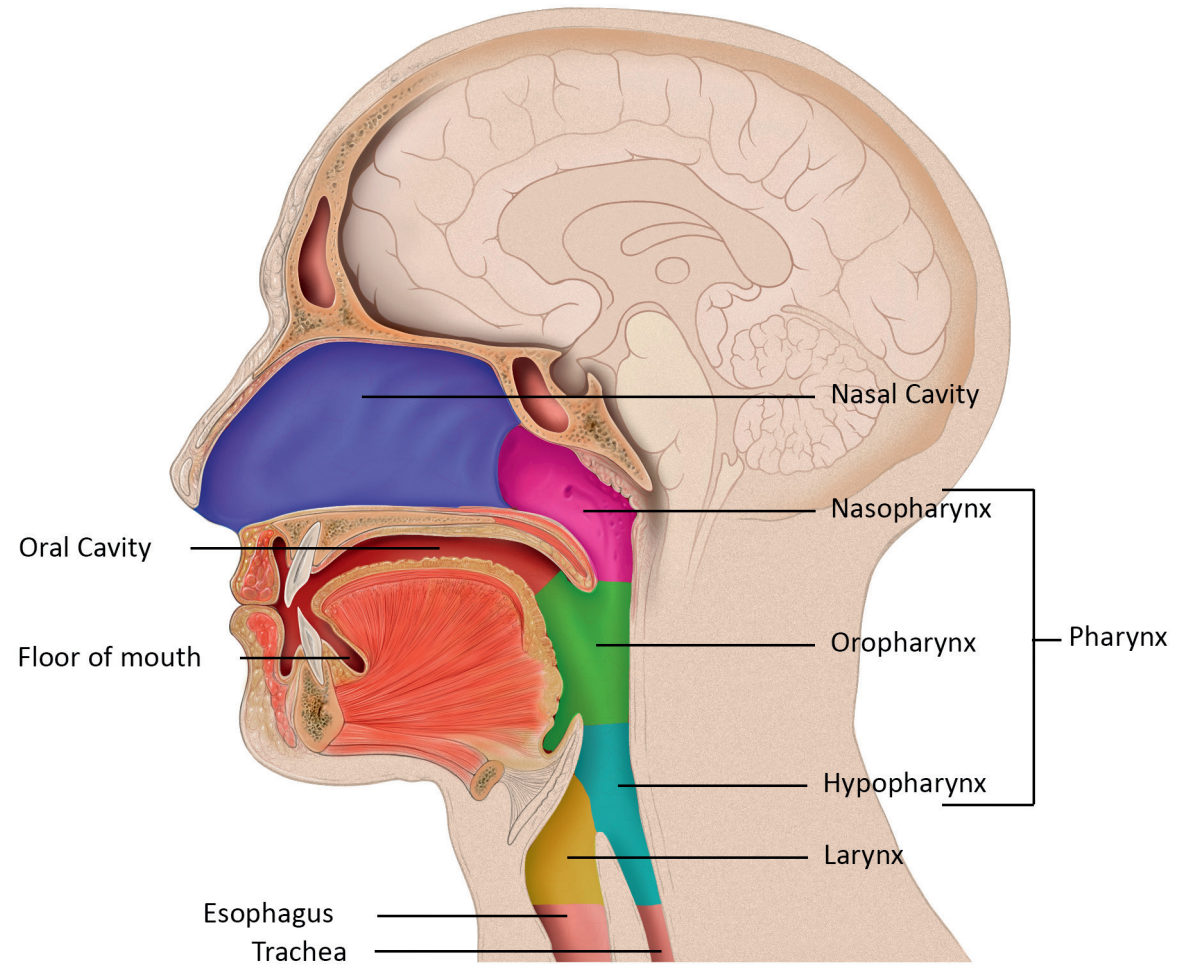

Figure 1.1 - Overview of head and neck regions, subdivided by color in the midsagittal plane. Adapted from Patrick J. Lynch, medical illustrator / CC BY (https://creativecommons.org/licenses/by/2.5) 

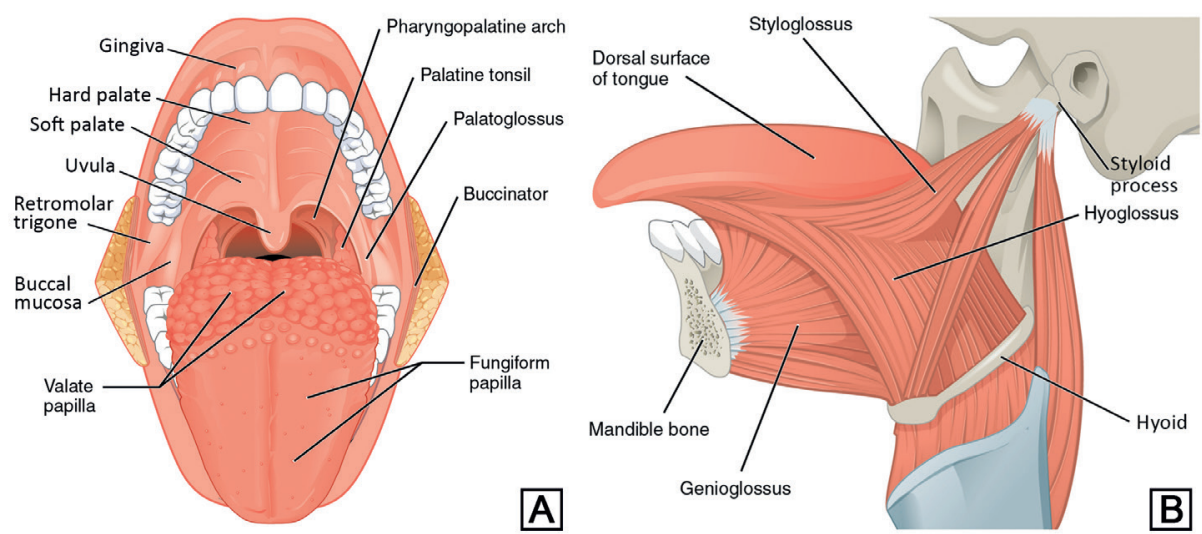

Figure 1.2 - Anatomy of the Oral cavity, frontal view (A) and extrinsic muscles, side view (B). OpenStax / CC BY (https://creativecommons.org/licenses/by/3.0)

The tongue is a muscular hydrostat, meaning that the tongue has no skeletal support and instead relies on the incompressibility of water at physiological pressures to change its shape [8]. It is enveloped in mucosa which are supplied by multiple sensory nerves: the lingual nerve for sensation, the chorda tympani for taste in the anterior two-thirds of the tongue, and the glossopharyngeal nerve for both taste and sensation in the rest of the tongue [9]. The tongue consists of four intrinsic and four extrinsic muscles (Table 1.1). The extrinsic muscles connect the tongue to external structures, while the interdigitating intrinsic muscles mainly contribute to the shape of the tongue. The intrinsic muscles include the transverse muscle, vertical muscle, superior, and inferior longitudinal muscle. These muscles are mainly responsible for large internal deformations of the tongue (Figure 1.3). The extrinsic muscles are the genioglossus arising from the mandible (jaw), the styloglossus from the styloid process of the temporal bone, the palatoglossus from the palatine aponeurosis, and the hyoglossus from the hyoid bone (Figure 1.2b) [10]. Other muscles attached to, but not part of the tongue, are the digastricus, mylohyoid, and geniohyoid muscle [10]. These muscles are also taken into account when analyzing the motion of the tongue in biomechanical modeling (paragraph 0 ).

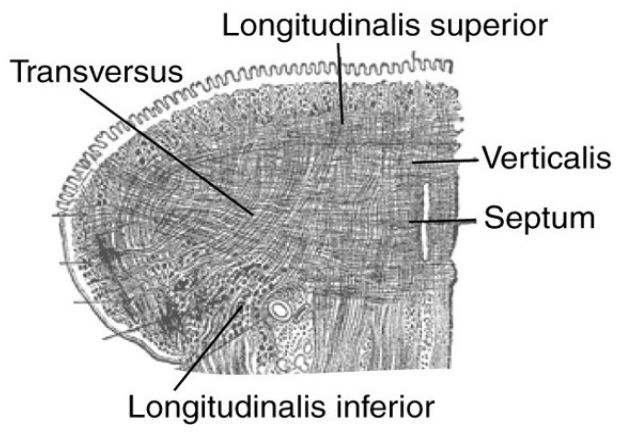

Figure 1.3 - Coronal view of the intrinsic muscles. Henry Gray (1918) Anatomy of the Human Body. 
While the shape and size of the tongue vary significantly among individuals, the muscular arrangement in humans seems to follow a strict pattern [11,12]. In contrast, innervation displays a different pattern. The hypoglossal nerve (XII) is a motor nerve that innervates all muscles of the tongue, except for the palatoglossus, which is innervated by a motoric branch of the vagal nerve. The left and right XII enter the tongue ventrolaterally to the posterior part of the tongue [11]. The first muscle it innervates is the hyoglossus, after which it is divided into the a lateral (I-XII) and medial ( $\mathrm{m}$-XII) branches to innervate other tongue muscles. The I-XII has two different types of possible topologies: single branching (40\%) and multiple branching (60\%) [11]. However, previous research by our group showed that specific global branching topology is not limited to characteristic muscle activity [13]. Even in a single patient, both sides of the tongue can have different distal branching topologies, resulting in different muscle activations on a microlevel [13]. It is assumed that topology influences functional outcome after surgery.

Table 1.1 - Muscles of the tongue and their type, abbreviation, and actions [14].

\begin{tabular}{|c|c|c|c|}
\hline $\begin{array}{l}\text { Muscle } \\
\text { type }\end{array}$ & Muscle name & abbreviation & Action \\
\hline \multirow{4}{*}{$\begin{array}{l}\overline{\overline{+}} \\
\overline{\bar{\Xi}} \\
\overline{\bar{n}} \\
\stackrel{\bar{n}}{n} .\end{array}$} & Superior longitudinal & SL & Broadens, retracts, elevates apex \\
\hline & Inferior longitudinal & IL & Broadens, retracts, lowers apex \\
\hline & Transverse muscle & TRA & Elongates, narrows \\
\hline & Vertical Muscle & VER & Elongates, broadens \\
\hline \multirow{4}{*}{ 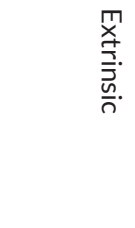 } & Genioglossus & GG & $\begin{array}{l}\text { Depresses, protrudes, deviates } \\
\text { contralaterally }\end{array}$ \\
\hline & Hyoglossus & HG & Depresses, retracts \\
\hline & Styloglossus & STY & Elevates lateral, retracts \\
\hline & Palatoglossus & PG & Elevates Root, constricts the isthmus \\
\hline \multirow{3}{*}{ 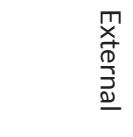 } & Digastricus & DG & Elevates hyoid bone, depresses the mandible \\
\hline & Mylohyoid & $\mathrm{MH}$ & Elevates hyoid bone and tongue. \\
\hline & Geniohyoid & $\mathrm{GH}$ & Elevates and protrudes hyoid bone \\
\hline
\end{tabular}

\subsection{EPIDEMIOLOGY}

In the international epidemiological literature describing the global incidence of oral cancer, oral and oropharyngeal cancer are often grouped together. Worldwide, oral and oropharyngeal cancer account for an estimated 447,751 new cases a year, resulting in 228.389 deaths in 2015 [15-17]. They represent the $8^{\text {th }}$ most common cancers in the world, constituting $2.5 \%$ of all cancer cases. 
Roughly $80 \%$ of this combined group of oral and oropharyngeal cancers is made up of oral cancers. The Age-Standardized Rates of this cancer are 5.8 in men and 2.3 in women per 100,000 people worldwide, but these figures differ significantly among regions and age groups.

The majority of oral cancers occur in low-income countries, concentrated in South Asia [15-17]. In Europe, high incidence rates of 9 and 10 per 100,000 are found in Central and Eastern Europe, however, two southern European countries, France and Portugal, rank at the top the list [18]. In the Netherlands, the estimated incidence rate of oral cavity cancer was 4.9 per 100,000 in 2019, with a 5-year survival rate of $61 \%$, which has not substantially increased in the past 50 years (Figure 1.4). Of all oral cavity cancers, 39\% are tongue cancer [19].

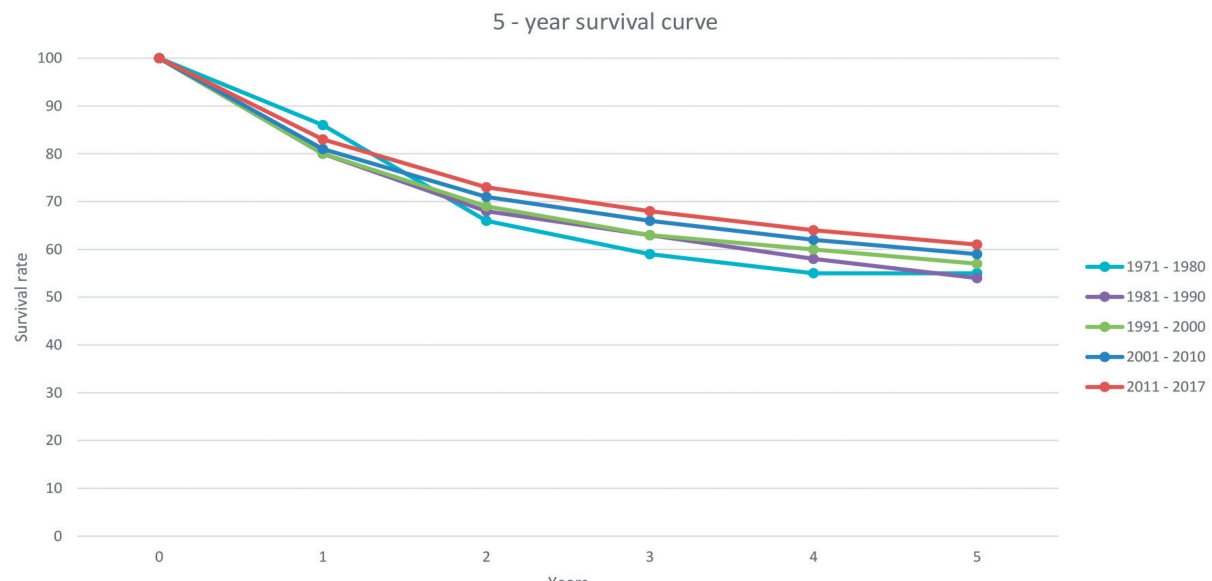

Figure 1.4 - The 5-year survival rate of tongue cancer in the Netherlands for different periods of 10 years, starting in 1971 [19].

In Europe, alcohol abuse and smoking are the main risk factors for oral cancer, and these also have a synergetic effect [20]. Other risk factors include smokeless tobacco use and betel quid chewing (common across India and other parts of Asia), psoriasis, and conditions associated with immune deficiency or dysregulation [21-23]. Head and neck cancers, in general, appear to be more common when a first-degree relative had the same type of cancer [24]. A genetic component is also therefore likely. In patients suffering from Fanconi anemia, a rare, hereditary disease, the chances of developing oral cancer are increased 500-fold [25]. For the majorityoforalcancercases, however, ageneticbasishasnotyetbeenfound [26,27].

Squamous cell carcinoma can also have a viral origin. The incidence of Human papillomavirus (HPV) induced squamous cell carcinoma (SCC) (which is also responsible for cervical cancer) has increased significantly in the past decade. This is mainly due to the increase in HPV-induced oropharyngeal cancer, which 
is responsible for $70 \%$ of the cases, and this number is still on the rise, mainly in middle-aged men [28-32]. While SCC in the oral cavity can also be induced by $\mathrm{HPV}$, the incidence remained the same or has even declined in the last decade $[30,33,34]$. HPV-induced SCC of the oropharynx has a better survival outcome than non-HPV induced SCC, less chance of recurrent disease, and responds better to radiotherapy [35-37]. HPV-induced oral cancer is not associated, however, with a better survival outcome [38].

\subsection{DIAGNOSIS AND TREATMENT}

\section{Clinical presentation}

$90 \%$ of all oral cancers are squamous cell carcinomas (SCC) [39]. The vast majority of these cancers are located in the tongue, mainly arising at the lateral border [40]. Symptoms include pain, nonhealing ulcers, dysphagia, and odynophagia. Tongue cancer may present with dysarthria and swallowing problems in advanced cases. Between $0.13 \%$ and $34.0 \%$ of cases arise from dysplastic premalignant mucosal lesions like leukoplakia or erythroplakia [41,42]. Leukoplastic and ulcerated areas usually also result in a loss of mucosal elasticity [43].

\section{Staging}

The Tumor, Node, Metastases (TNM) staging system was developed by the International Union for International Cancer Control (UICC) to classify malignancies (Table 1.2) [44,45]. Staging is a standardized clinical framework used by clinicians and researchers to choose treatment options, classify clinical and scientific research, and measure outcomes aimed at improving cancer control. The "T" represents the primary tumor status; " $N$ " represents the status of the regional lymph nodes; and " $M$ " indicates the presence or absence of metastatic disease.

The current $8^{\text {th }}$ edition of TNM also includes maximum invasion depth in addition to tumor size in a single plane to determine the T-status of tongue cancer [46]. A separate classification is also added to demarcate HPV-positive tumors occurring mainly in the oropharynx. Prognostic stage groupings and survival rates can be derived from the respective TNM stages and are shown in Table 1.3 [47]. 
Table 1.2 - TNM stages and their definitions

\begin{tabular}{|c|c|}
\hline TNM stage & \\
\hline T1 & Tumor $\leq 2 \mathrm{~cm}$ with depth of invasion $(\mathrm{DOI})^{*} \leq 5 \mathrm{~mm}$ \\
\hline T2 & $\begin{array}{l}\text { Tumor } \leq 2 \mathrm{~cm} \text {, with } \mathrm{DOI}^{*}>5 \mathrm{~mm} \text { and } \leq 10 \mathrm{~mm} \text {; or } \\
\text { Tumor }>2 \mathrm{~cm} \text { and } \leq 4 \mathrm{~cm} \text {, with DOI* } \leq 10 \mathrm{~mm}\end{array}$ \\
\hline T3 & $\begin{array}{l}\text { Tumor }>2 \mathrm{~cm} \text { and } \leq 4 \mathrm{~cm} \text { with DOI* }>10 \mathrm{~mm} \text {; or } \\
\text { Tumor }>4 \mathrm{~cm} \text { with DOI* } \leq 10 \mathrm{~mm}\end{array}$ \\
\hline $\mathrm{T} 4 \mathrm{a}$ & $\begin{array}{l}\text { Tumor }>4 \mathrm{~cm} \text { with } \mathrm{DOI}^{*}>10 \mathrm{~mm} \text {; or } \\
\text { Tumor invades adjacent structures only }\end{array}$ \\
\hline $\mathrm{T} 4 \mathrm{~b}$ & Tumor invades masticator space \\
\hline cN1* & Metastasis in a single ipsilateral lymph node, $3 \mathrm{~cm}$ or smaller \\
\hline cN2a & $\begin{array}{l}\text { Metastasis in a single ipsilateral node larger than } 3 \mathrm{~cm} \text { but not larger than } 6 \mathrm{~cm} \text { in } \\
\text { greatest dimension }\end{array}$ \\
\hline $\mathrm{cN} 2 \mathrm{~b}$ & $\begin{array}{l}\text { Metastases in multiple ipsilateral lymph nodes, none larger than } 6 \mathrm{~cm} \text { in greatest } \\
\text { dimension and/or }\end{array}$ \\
\hline $\mathrm{cN} 2 \mathrm{c}$ & In bilateral or contralateral lymph nodes, none larger than $6 \mathrm{~cm}$ in greatest dimension \\
\hline cN3a & Metastasis in a lymph node larger than $6 \mathrm{~cm}$ in greatest dimension \\
\hline cN3b & Metastasis in any node(s) and clinically overt \\
\hline $\mathrm{M0} / \mathrm{M} 1$ & (no) distance metastasis \\
\hline
\end{tabular}

${ }^{*}$ Simplified version: within TNM the $\mathrm{N}$ stages are different for clinical $\mathrm{N}$-stage $(\mathrm{cN})$ and pathological $\mathrm{N}$-stage $(\mathrm{pN})[46]$.

Table 1.3 - Prognostic stage, corresponding TNM stage, and baseline 5-year survival of oral cavity cancer [47].

\begin{tabular}{lllll}
\hline Prognostic stage & Tstage & N stage & M stage & Baseline 5-year survival \\
\hline Stage 0 & Tis & N0 & M0 & $100 \%$ \\
\hline Stage I & T1 & N0 & M0 & $95 \%$ \\
\hline Stage II & T2 & N0 & M0 & $90 \%$ \\
\hline Stage III & T3 & N0 & M0 & $89 \%$ \\
\hline & T1 & N1 & M0 & $93 \%$ \\
\hline & T2 & N1 & M0 & $87 \%$ \\
\hline Stage IVA & T3 & N1 & M0 & $85 \%$ \\
\hline & T4a & N0 & M0 & $85 \%$ \\
\hline & T4a & N1 & M0 & $81 \%$ \\
\hline & T1 & N2 & M0 & $91 \%$ \\
\hline & T2 & N2 & M0 & $83 \%$ \\
\hline Stage IVB & T3 & N2 & M0 & $81 \%$ \\
\hline & T4a & N2 & M0 & $75 \%$ \\
\hline Stage IVC & T4b & N3 & M0 & $69 \%-88 \%$ \\
\hline
\end{tabular}




\section{Treatment}

Following the Clinical Practice guidelines in Oncology by the National Comprehensive Cancer Network (NCCN) , the preferred treatment of T1 and T2 tumors of the mobile tongue is surgery, followed by re-excision or adjuvant radiotherapy in case of incomplete resection [48]. Superficially growing T1 and T2 lesions can also be treated by Photodynamic therapy (PDT) [49]. Defects after removal of T1 and T2 lesions are often closed primarily, whereas defects after removal of T3 and T4 lesions are reconstructed using revascularized free flaps. The fasciocutaneous radial forearm flap, known for its pliability, is often used in such cases. Advanced T3 and T4 lesions, which are considered to be functionally inoperable, can be treated with organ sparing concurrent chemoradiation $[48,50]$.

Immunotherapy has revolutionized the treatment of multiple cancers and is now also being used in head and neck squamous cell carcinomas (HNSCC) [51]. As compared to other systemic cancer therapies, immunotherapy has the distinct advantage in that its effects can be long-lasting, prolonging life for more than 5 years in some cases of non-small cell lung cancer [52]. While it proved to be an immense success in some of patients, the vast majority (80\%) of HNSCC do not respond to combinations of immunotherapy that have recently been evaluated [51]. New combinations of immunotherapy and finding biomarkers for patient selection have the potential to improve these numbers [51,53]. Immunotherapy has the potential to treat cancer without surgical intervention, but until then, surgery remains the first line treatment.

\subsection{FUNCTIONAL CONSEQUENCES}

The tongue is a crucial organ for everyday life, as it contributes significantly to eating ability and speech quality. Impaired function after treatment of tongue cancer negatively affects quality of life (QOL) $[54,55]$. The size and location of the tumor determine the grade of function loss after treatment [56-60].

Problemswithswallowingandmasticationcanleadtomalnutrition, depression,loss of employment, and in combination with speech defects also lead to social isolation $[22,61,62]$. As many as $52 \%$ of patients treated for head and neck tumors are unable to work after treatment, often leading to more psychosocial problems [63-65].

All functions of the tongue rely on complex combined actions of muscles actuated by nerves and require years of training, mainly in the first years after birth [66]. The removal of tissue and muscles after a surgical intervention changes the way the muscles need to be controlled to create specific tongue shaping. This neuromuscular fine-tuning explains in part the serious impact of surgery and/or chemoradiation on tongue function. Although large defects can be reconstructed 
by free revascularized tissue flaps, achieving restoration of function in these transplants remains extremely difficult [67].

Surgically induced neural damage can have multiple effects on postoperative function [62]. However, due to the versatility of the tongue in combination with bilateral innervation by the hypoglossal nerves, a high degree of compensation can be achieved. Function loss by unilateral damage of the hypoglossal nerve can be compensated, for instance, by innervation from the contralateral nerve $[68,69]$. Dysarthria after partial glossectomy is mainly determined by the extent of surgery or radiation-induced fibrosis and predominantly affects contralateral movements [70,71]. Damage to the sensory nerves leads to the inability to sense and taste, often without significant effects on oral proprioception [72].

The mobile tongue, in particular the genioglossus, hyoglossus, and geniohyoid muscle compartments, are important for speech and swallowing, whereas the base of the tongue is more involved in swallowing [4,73]. Speech intelligibility and swallowing activity, however, are also dependent on the intactness of the other mucosal linings of the upper aerodigestive tract [59,74]. Apart from tongue defects, surgical defects of the hard and soft palate may also have a significant impact on the intelligibility and swallowing.

After treatment, speech intelligibility may not be seriously affected in an objective evaluation based on intelligibility measurements e.g. by a speech pathologist. Social perception of a speaker's voice, however, is quite sensitive and is often negatively impacted even with minor impairments [75]. This means that even without serious, objectively measured functional consequences, the psychological consequences for the patient may still be significant. Organ-sparing alternatives for advanced tongue cancer, such as chemoradiation, are not necessarily better in terms of functional outcomes [76-78]. Radiation-induced fibrosis, xerostomia, mucositis, and necrosis can have negative effects on swallowing and to a lesser extent on speech. Choosing between two curative treatment options poses a dilemma for the treating physician.

The choice is less difficult, however, in the case of anatomical inoperability. In this situation, the patient would not be able to survive the treatment. This is seen, for example, when a tumor invades the base of the skull or when sacrificing the internal carotid artery is unavoidable for complete resection of the tumor in combination with a high risk of developing hemiplegia due to this procedure. More difficult decisions are encountered when resection of a tongue carcinoma would lead to an expected yet unacceptable functional loss of speech and swallowing. These cases are deemed to be functionally inoperable [4]. While the patient's opinion is very important in this process, the decision that they arrive at will also be highly 
influenced by information from the physician. However, as seen in Kreeft et al. [4], functional loss is indeed quite difficult to determine, as it is highly subjective and variable among patients. The survey conducted in one of Kreeft's [4] publications showed that half of surgeons judged a total glossectomy or mandibulectomy as functionally inoperable, whereas for other procedures there was at best a weak consensus, with the exception of glossectomy in combination with a supraglottic laryngectomy.

\subsection{THE DIGITAL TWIN}

In response to this lack of an objective way to predict function loss, the Virtual Therapy group was established in 2010 . This project aims to provide reliable tools to base treatment plans on standardized, objective, and accurate data. The availability of this data during a multidisciplinary meeting could help achieve consensus among physicians by weighing objectively determined functional loss. Based on this information, patient counseling could be improved: showing the expected effects of different treatment options would help the patient in their own decision-making. These discussions, based on objectively determined data, would also help manage expectations for both the clinician and the patient.

A regular workflow for a patient with tongue cancer starts with the consult (Figure 1.5) during which the patient is clinically examined and additional imaging is performed. The examinations are then discussed in the multidisciplinary meeting to determine the definitive staging and to formulate the treatment plan. A decision is made about either the anatomical inoperability or functional inoperability. Following the determinations made by the multidisciplinary board, the patient will be informed about the proposed treatment or treatments by the treating physician as well as the expected functional loss of the procedure(s). In this workflow, no patient-specific data is used actually to assess the expected functional loss, as the information is based on standardized data. As discussed previously, the effects of a certain treatment can indeed differ from person to person, and this must be kept in mind.

The Virtual Therapy project aims to assist with personalized visual predictions of the expected post-treatment function of the upper aerodigestive tract using a "Digital Twin model". Using interactive audio and visual modelling, this prediction model depicts post-treatment mastication, swallowing, and speech based on the simulated treatment. It enables the physician to simulate various treatments, but also different techniques for performing a treatment. In the case of surgery, the functional consequences of primary suturing or free flap reconstruction can be modelled. In the case of chemoradiation, the radiation fields can be implemented to show the functional effects of fibrosis. These visualizations 
will ideally contain simulations of swallowing using simulated food of different consistencies. Movements of the tongue needed for mastication can be depicted, and postoperative speech can be synthesized and made audible.

Such a system can help guide a multidisciplinary board to objectively judge the functional loss in complex clinical cases and subsequently assist the patient in coming to his or her choice regarding treatment (Figure 1.5). Even in cases where the tumor is functionally inoperable, it could still assist in managing expectations for the patient.

Treatment planning might need to be adapted due to unexpected outcomes, complications, or new insights. Accordingly, this Digital Twin is meant to be updated with every step of the clinical workflow. This helps not only to manage patient expectations, but also to provide physicians with information on the effects of certain treatment decisions in hindsight. Maintaining this data in a central data structure creates a rich database from which new clinical information about functional sequelae can be obtained.

The model can also play a role after treatment. As a result of both the disease progression and the accompanying treatment, the anatomy of the organ changes. To regain (part of) the original function, exercises under the supervision of a speech therapist are often needed. During these training sessions, it is often hard to predict which exercises will prove beneficial for the individual patient. This is because postoperative function is highly individual, depending largely on the remaining muscles, the developed fibrosis, and the innervation. While muscles are definitively removed, nerves can partly regenerate and remap over time, and the effect of (radiation-induced) fibrosis can sometimes be reduced by exercise $[79,80]$. The Digital Twin can simulate the compensatory movements that are still possible using the remaining muscle structures. A mismatch between the model and the patient shows the therapist that there is still function to be gained by exercise and which muscles could compensate for the loss of others. 


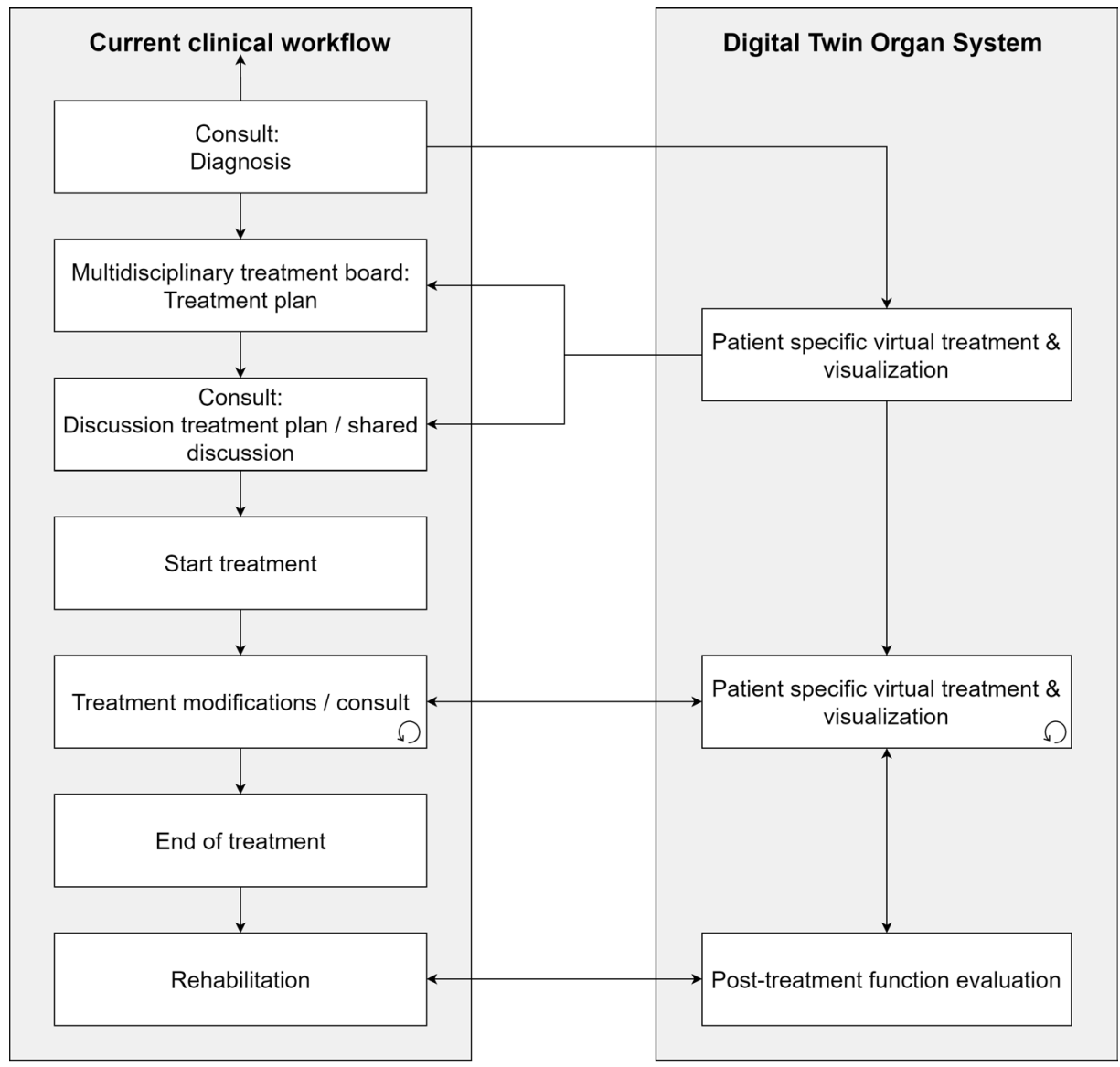

Figure 1.5 - A flowchart showing the current clinical workflow and the proposed parallel workflow using the Digital Twin. (Design: F. vd Heijden Ph.D., 2010)

The model can also play a role after treatment. As a result of both the disease progression and the accompanying treatment, the anatomy of the organ changes. To regain (part of) the original function, exercises under the supervision of a speech therapist are often needed. During these training sessions, it is often hard to predict which exercises will prove beneficial for the individual patient. This is because postoperative function is highly individual, depending largely on the remaining muscles, the developed fibrosis, and the innervation. While muscles are definitively removed, nerves can partly regenerate and remap over time, and the effect of (radiation-induced) fibrosis can sometimes be reduced by exercise $[79,80]$. The Digital Twin can simulate the compensatory movements that are still possible using the remaining muscle structures. A mismatch between the model and the patient shows the therapist that there is still function to be gained by exercise and which muscles could compensate for the loss of others. 
The head and neck region is a complex organ system where the risk of vital functional loss after cancer treatment is high. The same methodology is likely to work in less complex, but still risky interventions such as plastic and reconstructive surgery. When the Digital Twin is complete, it is expected that the methodology will be applied to other interventions that could benefit from treatment simulation.

The chapters of this thesis detail part of the creation of the Digital Twin and provide ground for the further development of the digital model. The oral cavity, and in particular the tongue, is without any doubt, the most complex part of the Digital Twin. This thesis will, therefore, focus primarily on the tongue.

\subsection{MEASURING TONGUE FUNCTION}

In recent years, head and neck cancer research has increasingly focused on evaluating remaining function or the regaining of function. A large part of this research is based on subjective measurements using quality-of-life questionnaires. The European Organization for research and treatment of cancer (EORTC) QLQ-C30, specifically the head and neck module (H\&N35), is the questionnaire most used [81,82]. Other frequently used questionnaires include The University of Washington QOL Questionnaire (UWQOL) [83], the functional assessment of cancer therapy - head and neck module (FACT-HN40) [84], and the University of Michigan Head and Neck QOL Questionnaire [85]. Among these, many other validated questionnaires are used to assess the physical, psychological, and socioeconomic effects of oral cancer and specific treatments.

While most questionnaires are good at converting a subjective entity into something measurable, they do not measure objective individual function loss. Several methods can be used to assess objective swallowing, mastication, and speech function. In a clinical setting, swallowing is usually measured by videofluoroscopy or endoscopy, and the quality of mastication is usually assessed by measuring the degree of breakdown of chewed food [86]. Speech is generally measured using perceptual analysis by a speech pathologist or specialized software [86]. While these methods are relatively easy and quick ways to score the function of an individual, they do not provide detailed information about the underlying tongue movement.

Speech, swallowing and mastication are a result of complex brain, nerve, and muscle interactions in which the tongue shape and mobility are very important. A measure for measuring the mobility of the tongue is the Range of Motion (ROM). Several techniques can be used to measure the ROM and the deformation of the tongue during certain tasks. In the literature, the ROM of the tongue is 
often measured by a simple ruler or commercial products such as the TheraBite measuring disc [87], sometimes using only a 3 -point scale [57,88,89]. To measure deformation, conventional imaging modalities such as videofluoroscopy and ultrasound can also be used to track the dorsal surface of the tongue [90]. Tagged cine MRI can also be used to track the position of internal points of the tongue to measure tongue deformation [91]. These modalities, however, are often only able to measure in 2D, which make them suitable for certain types of speech research, but lack the 3rd dimension to capture every detail of tongue movement. In addition, videofluoroscopy exposes the patient to radiation [92,93].

Electromagnetic articulography is often used to measure tongue movement and deformation in 3D and has the benefit that a line of sight with the sensors is not needed, so that the tongue can be tracked with the mouth closed [94,95]. It uses electromagnetic induction to measure the position and movement of different sensor coils placed in the mouth. However, the markers require a minimum distance from each other and can also interfere with speech production and other tongue functions. Beyond these issues, the markers are often difficult to attach and the device difficult to operate. Therefore, caution and experience are needed to obtain reliable measurements $[94,96]$.

A very promising technique is dynamic MRI, which has already been utilized to create 2D videos of the tongue in motion [97]. 3D dynamic MRI is currently in its infancy, but once it becomes more accessible it could prove to be the most promising technique for measuring tongue function [98].

Another objective technique to measure 3D motion is optical tracking. Using a 3D camera, tongue motion can be tracked while performing tongue movements. After the ruler, this is one of the most convenient and quick measurements for both patient and observer. However, optical tracking has one intrinsic flaw: it needs a line of view with the object that is tracked. For the tongue, this is complicated since the tongue is usually inside the mouth. Tracking the maximum ROM of the tongue this method, though, appeared to be reliable, non-invasive, and quick, as demonstrated in Van Dijk et al. [71], who created a 3-camera system to track the position of the tongue in 3D. In Chapter 4, we will present an improved version of the method using the same camera system.

Most measurement systems measure the output of the tongue caused by combined muscle interactions. Theoretically, sEMG enables measurements at the source: the muscle innervation. EMG measures the algebraic summation of motor unit action potentials and can assess the intracellular action potentials with relative ease. This was used in the Virtual Therapy project to predict the movement of the lips [99]. The challenge here is the mounting of the electrodes 
since the oral cavity is a wet environment and the sensors need to move with the tongue and remain in contact. A second challenge is to make these sensors as small as possible while still increasing the resolution. Few studies have succeeded in creating EMGs of the genioglossus [100-102]. Within the Virtual Therapy group, an EMG grid approach was attempted, but difficulties arose in trying to get a proper fixation while also incorporating enough electrodes [103].

\subsection{PREDICTING FUNCTION}

Because of the complexity of the tongue, a statistical prediction based on the T stage, size, or location is not enough to make a precise prediction on the functional outcome of the individual. Also, the number of patients with comparable types of tongue cancer (same location, same size) is limited, and therefore more parameters are hard to incorporate in statistical models.

To solve this problem, the Virtual Therapy project uses Finite Element (FE) simulations to predict postoperative function. The FE method is a numerical method that solves partial differential equations with boundary value constraints. To solve a complex mechanical problem, the object of interest (or domain) is subdivided into a finite number of simple parts: the so-called elements. The problem - whether this is a heat transfer problem, a flow problem, or a structural analysis problem - is much easier to solve for the smaller elements than for the domain as a whole. A set of scientific papers from the 1940s laid out the theoretical basis for FE modeling as we know it today [104,105] and the name "Finite Element Method" was coined by Ray W. Clough [106] in a 1960 article. While the method has been around some time, it only gained real attention when computers became significantly faster some two or three decades ago [107]. Since then, and with growing computing speed and power, the method has also begun to gain more traction in the medical field.

Another real change was observed when more commercial software became more widely available for the industry, boosting the use of FEM [107]. One of the open-source FEM solutions, ArtiSynth, is used extensively in Chapters 2, 5, and 6. Artisynth is an interactive biomechanical modeling toolkit that combines multibody and FE simulation [108]. An introduction to the working mechanisms of this software is described in Appendix A.

\section{Biomechanical models of the tongue}

While FE modeling has not been yet introduced on a wide scale in medical research, it can be implemented in numerous applications and solutions, e.g. to calculate the structural integrity or weak points of bones and implants in orthopedics $[109,110]$. It can simulate fluid dynamics and wall stiffness of vessels and arteries, 
or it can guide targeted ablation in case of persistent atrial fibrillation [111,112]. But it can also be used to simulate the motion of the tongue as a result of muscle contraction, opening the path towards the simulation of complex movements like speech, mastication, and swallowing [113-116].

While the idea of biomechanical modelling sounds futuristic and high tech, it might come as a surprise that the first biomechanical models of the tongue date back half a century ago. While biomechanical models of the tongue have existed for quite some time in various forms, to our knowledge Joseph Perkell was the first to truly describe a physically oriented model, in 1974 [117] (Figure 1.6). His new methodological approach provided the basis of considerable amount of subsequent research on the relationship between phonetic models and the mechanical properties of the tongue and other parts of the orofacial motor system [117-119].

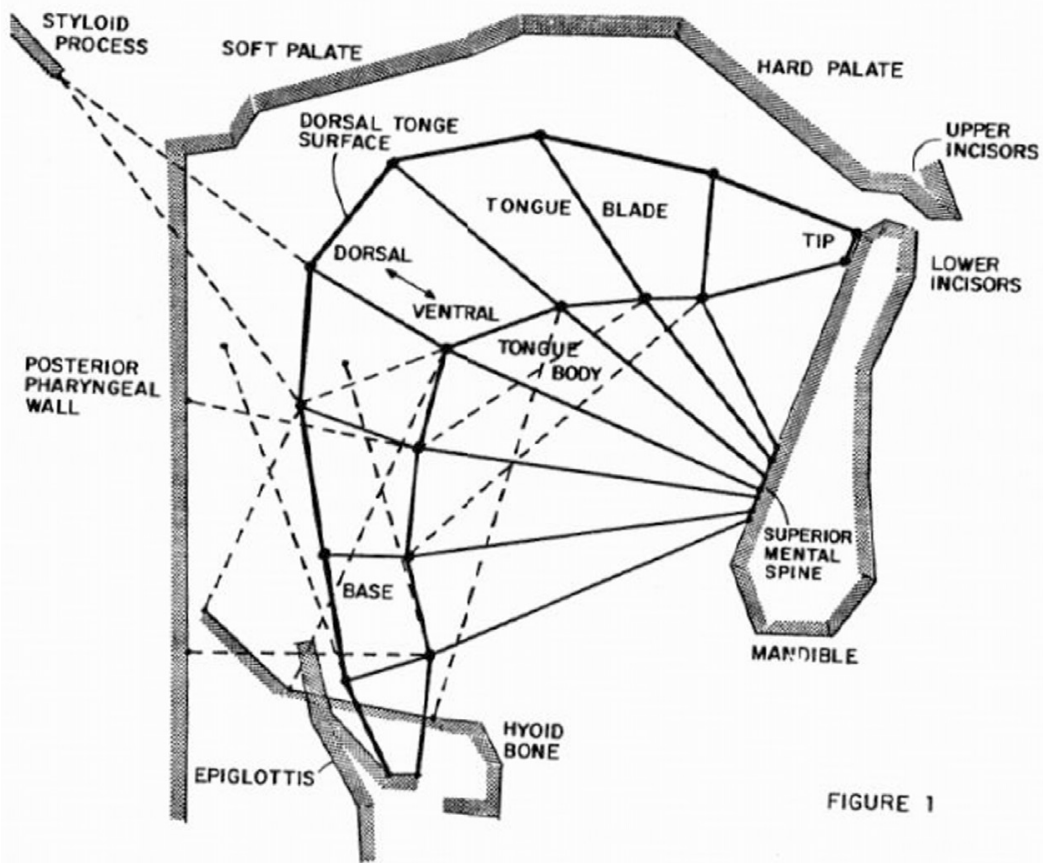

Figure 1.6 The first description physically oriented model in the thesis of Joseph Perkell [117]. Obtained with permission from the Massachusetts Institute of Technology.

In the 25 years that followed, various models that attempted to challenge the complexity of rendering realistic deformation in speech production and other functions of the tongue [120-124] were created. In most of these works, the FE method was adopted to simulate deformation in 2D. Following the trend 
of increased computing power, twenty-first century models became more sophisticated and complex, and transitioned from 2D FEM to 3D FEM modeling.

In 2005, Gérard et al. [125] elaborated on the work of Wilhelms-Tricarico [126] by creating an FE model based on MRI data and information about muscle anatomy from the Visible Human Project [127]. In turn, Vogt et al. [128] and Buchaillard et al. $[114,129]$ further built upon on this model by exploring the simulation of speech as well as surgery $[114,128,129]$. Based on the manual segmentation of MRI and CT images and the work of Rohan et al. [130], Hermant et al. [131] adapted and improved the model that has been evolving since 2000. Also within our research group, Van Alphen et al. [113] created a FE model to show the effects of impairments on the movement of the tongue.

As for simulating surgery in biomechanical models, the attempts are fairly limited. Both Buchaillard et al. [129] and Fujita et al. [132] created tongue models, of a specific subject, specifically for the simulation of a partial glossectomy. Both studies explored the effects of surgery using a free-flap approach, by stiffening parts of the tongue model.

In most stage T1-T2 tumors, primary closure is the most common surgical technique, and this cannot be simulated realistically by just stiffening parts of the model. In Chapters 3 and 6, we go into greater detail on this problem and propose a method to simulate surgery using primary closure.

\section{Personalization of biomechanical models}

Personalization of a biomechanical model might sound straightforward, but is it? Every human is different, and so is every tongue. The position relative to the oral cavity, volume, force, length, and width are all measurable aspects of tongue anatomy. Muscle anatomy, stiffness, innervation patterns, motor units, and even muscle mapping in the motor cortex are factors that also influence tongue function. Many of the models discussed in the previous paragraph are partially 'personalized' in the sense that they are only based on the segmented shape from an MRI of a particular subject. These models are built for one subject, and so analyzing another subject would require a new model. If we want to introduce a DDigital Twin model in clinical practice that can predict expected functional consequences, the process of personalization needs to be more comprehensive yet also quick and not dependent on intensive input from the clinician. The main goal of automated personalization is to create a method that includes all these necessary aspects and is robust enough to create a truly personalized model with only limited input required from the clinician. 
To personalize the muscular structure of a tongue model, obtaining patient data from imaging modalities is key. To create a 3D model of the tongue, segmenting MRI images is currently the most straightforward approach. Various techniques have been proposed to morph generic FE models based on the segmentation of MRI images [133,134]. The morphing of generic FE models was, for example, used to personalize skeletal muscles $[135,136]$ and faces of 60 individual subjects [137]. Binary masks or segmented data from MRI can also be used to calculate displacement fields applicable to a generic FE model $[138,139]$. The previously mentioned papers use a fully defined FE model from which elements will be morphed towards individual imaging data. Another way to morph is to start with the imaging data and automatically generate the FE model to match the shape of the segmented image. These techniques often embed a mesh and muscle structure into a coarse FE structure [140,141]. However, these techniques do not take into account the internal muscle structures of the tongue.

Diffusion-weighted MRI is a technique that can be used not only to personalize the outer shape of the tongue, but also the muscle bundles. This is a technique best known for visualizing the nerve tracts or white brain matter. Diffusionsensitizing gradients can be used to encode diffusion information from MR images in certain directions. Because water diffuses mainly along the direction of muscle fibers, the diffusion tensor enables the reconstruction of the fiber and its orientation [142]. In diffusion-tensor imaging (DTI) these orientations are described as tensors from which the first 'eigenvector' corresponds with the direction of the muscle fiber. Using a technique called tractography, the vectors of individual voxels are connected via streamlines to create 3D reconstructions of the muscle fibers (Figure 1.7). DTI is a reliable technique that is often mentioned as the next step in tongue model personalization $[5,143,144]$ and can be used as input for biomechanical models [145]. 


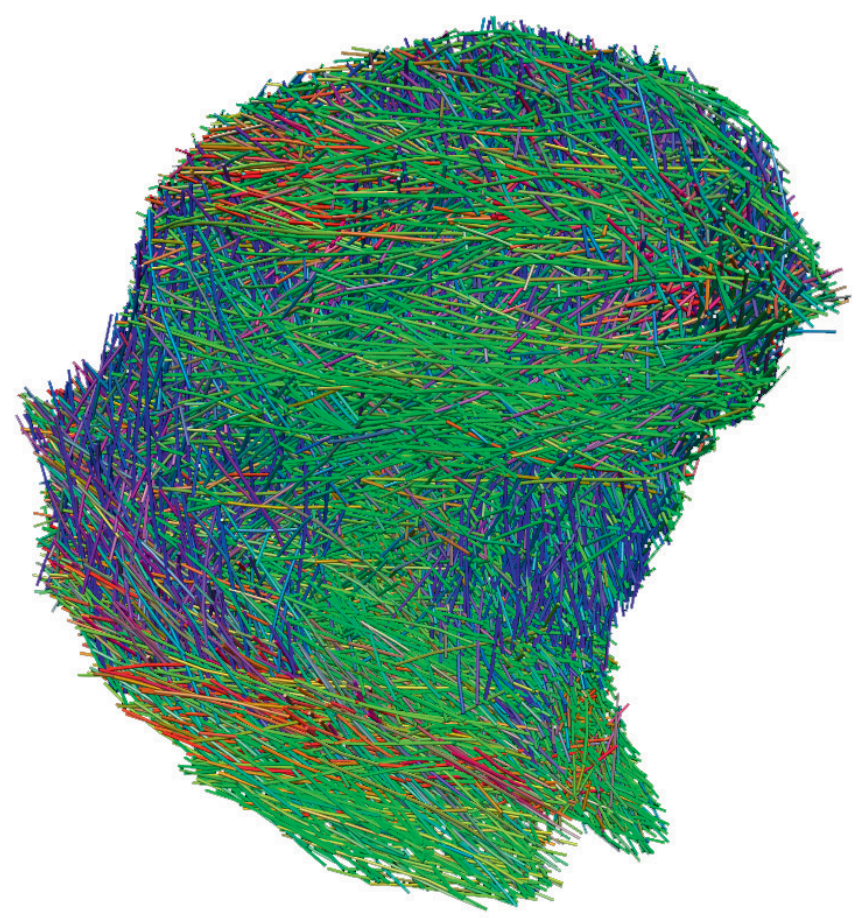

Figure 1.7 - Colored tracks visualizing the direction of muscle fibers acquired using a DTI MRI scan. Courtesy: L. Voskuilen MSc.

DTI has been used to visualize the tongue musculature in-vivo. However, it is constrained by the fact that it can only detect one muscle fiber per voxel [146]. This constraint was also apparent in studies that performed DTI following a partial glossectomy [147,148]. As mentioned in Chapter 1.1, the tongue consists of many interdigitated fibers that cannot be visualized using DTI. Higher-order models, such as Constrained Spherical Deconvolution (CSD) can resolve these crossing fibers [149]. Voskuilen et al. [150,151] applied this technique for the invivo human tongue and successfully visualized crossing fibers, confirming findings from anatomical studies. Chapter 6 will go deeper into the first biomechanical models that use CSD as a means of personalization.

\section{Tissue properties}

To simulate the deformation of a material, a description is needed of how the material reacts when it endures forces. In continuum mechanics, this relation between stress and strain is described by a so-called constitutive equation. In biological tissue, these quantities are usually stress and deformation. The constitutive equation is typically a phenomenological model that depends on unknown constants that should be identified by conducting experiments on a tissue. When, for example, stretching a uniform bar with a cross-section area of 
$A$ and length $L_{0}$ by $\Delta L$, with uniaxial force $F$, it will first deform elastically (Figure 1.8 between $A$ and $B$ ). After the elastic limit $C$, plastic deformation occurs followed by the breaking point $\mathrm{D}[152]$.

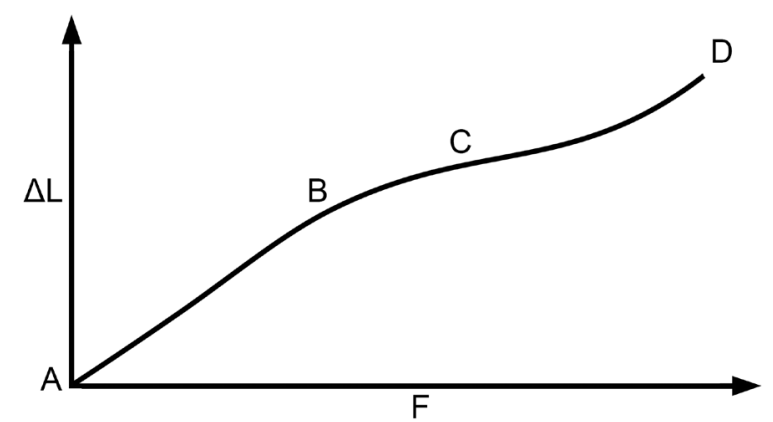

Figure 1.8 - Description of a typical relation between force

$F$ and change in length $\Delta L$ of a bar. A is zero, $\mathrm{B}$ is the proportional limit, $\mathrm{C}$ is the elastic limit, and $\mathrm{D}$ is the breaking point.

For now, we are only interested in the elastic (linear) part (A-B). This can be explained by Hooke's law [152]:

$$
F=k \Delta L
$$

$k$ is defined as:

$$
k=\frac{E A}{L_{0}}
$$

Here $E$ is the elastic or Young's modulus and it defines the relationship between stress and strain in a material:

$$
E=\frac{\sigma}{\varepsilon}
$$

where $\sigma$ is the stress $\left(\mathrm{Pa}=\mathrm{N} / \mathrm{m}^{2}\right)$ and $\varepsilon$ is the strain, i.e. relative displacement. Such a linear relation is too restrictive for most biological tissues that display nonlinear behavior. As the tongue consists of materials that can show nonlinear, time-dependent, inhomogeneous, and anisotropic behavior, it is better described in theoretical terms with a non-linear anisotropic viscoelastic model [153].

Due to the complexity of such models, however, many models published in the last decade, including the models in Chapters 1, 5, and 6, describe tongue tissue using a hyperelastic model $[114,116,122,125,154-156]$. Hyperelastic materials are usually used to describe rubber-like materials that exhibit non-linear elasticity 
and are incompressible and isotropic. Different hyperelastic models can be used to describe the behavior of tissue in the form of a strain energy density function (Table 1.4). This strain-energy density is the potential energy per volume element that is stored when the material undergoes a local deformation.

In case of the example above, the strain energy density would be $W=\frac{1}{2} \varepsilon \sigma=\frac{1}{2} E \varepsilon^{2}$. Thus, knowledge of $W$ as a function of $\epsilon$ implies knowledge of the material property $E$. In the nonlinear 3D case, the situation is more complicated. Here the material property is given by defining $W$ as a function of the so-called invariants $I_{m}$ of the right Cauchy-Green deformation tensor [157]. The first three invariants are as follows:

$$
\begin{gathered}
I_{1}=\lambda_{1}^{2}+\lambda_{2}^{2}+\lambda_{3}^{2} \\
I_{2}=\lambda_{1}^{2} \lambda_{2}^{2}+\lambda_{2}^{2} \lambda_{3}^{2}+\lambda_{3}^{2} \lambda_{1}^{2} \\
I_{3}=\lambda_{1}^{2} \lambda_{2}^{2} \lambda_{3}^{2}
\end{gathered}
$$

$\left(\lambda_{i}\right)_{i=1,3}$ are a series of so-called stretch ratios. They describe the deformation in certain principal directions.

The most commonly used material models (Table 1.4) are derived from the strain energy density function for an incompressible neo-Hookean material:

$$
W=C_{1}\left(I_{1}-3\right)
$$

$C_{1}$ is a material constant and is related to the elastic modulus (Table 1.4).

Different constitutive equations mimic different kinds of materials. Most rubbery materials under moderate strain (30\% to $70 \%$ ) are well represented by the neoHookean model. To model biological tissue at higher strains, the Mooney-Rivlin model is a common choice at present because it adds an additional constant to the neo-Hookean model and therefore better fits experimental data. The Ogden model is also well suited or modeling rubbery and biological materials at higher strains [157]. 
Table 1.4 - Overview of different constitutive models for biological tissue and their strain energy density functions.

\begin{tabular}{|c|c|c|c|}
\hline Model & Strain energy density function & & $\begin{array}{l}\text { Equivalent } \\
\text { Young's modulus }\end{array}$ \\
\hline $\begin{array}{l}\text { Neo- } \\
\text { Hookean }\end{array}$ & $W=C_{1}\left(I_{1}-3\right)$ & $\begin{array}{l}\text { With } I_{1} \text { the first variant of the } \\
\text { Cauchy-Green strain tensor, } C_{1} \\
\text { a constant. }\end{array}$ & $E=6 C_{1}$ \\
\hline Yeoh & $W=C_{10}\left(I_{1}-3\right)+C_{20}\left(I_{1}-3\right)^{2}$ & $\begin{array}{l}\text { With } I_{1} \text { the first variant of the } \\
\text { Cauchy-Green strain tensor, } C_{x} \\
\text { the material parameters. }\end{array}$ & $E=6 C_{10}$ \\
\hline $\begin{array}{l}\text { Mooney - } \\
\text { Rivlin }\end{array}$ & $W=C_{1}\left(\bar{I}_{1}-3\right)+C_{2}\left(\bar{I}_{2}-3\right)$ & $\begin{array}{l}\text { A polynomial series with } \bar{I}_{1} \\
\text { and } \bar{I}_{2} \text { the first and second left } \\
\text { Cauchy-Green deformation } \\
\text { tensor, } C_{1} \text { and } C_{2} \text { the material } \\
\text { parameters. }\end{array}$ & $E=6 C_{1}$ \\
\hline Gent & $W=-\frac{\mu J_{m}}{2} \ln \left(1-\frac{I_{1}-3}{J_{m}}\right)$ & $\begin{array}{l}\text { With } \mu \text { as the shear modulus. } \\
J_{m} \text { is the stiffening parameter } \\
\text { and its maximum value is of } \\
\left(I_{1}-3\right)\end{array}$ & $E=3 \mu$ \\
\hline Ogden & $W=\frac{\mu_{1}}{\alpha_{1}}\left(\lambda_{1}^{\alpha_{n}}+\lambda_{2}^{\alpha_{n}}+\lambda_{3}^{\alpha_{n}}-3\right)$ & $\begin{array}{l}\text { Where }\left(\lambda_{i}\right)_{i=1,3} \text { are the } \\
\text { principal stretches, and } \\
\mu_{1} \alpha_{1}>0 . \text { With } \alpha_{1} \text { as the } \\
\text { stiffening parameter. }\end{array}$ & $E=\frac{3 \alpha_{1} \mu_{1}}{2}$ \\
\hline
\end{tabular}

The constants that define the characteristics of the material can be estimated numerically or can be determined via experimentation using, for instance, a tensile test. Parameters based on real experimentation are scarce because of the difficulties entailed with measuring on the tongue. According to Hermant et al. [131], only three papers have been published about constitutive parameters that were found using in-vivo [158,159] or ex-vivo [125] experiments. The ex-vivo experiment by Gerard et al. [125] assumed a Yeoh 2-parameter strain energy function (Table 1.4) to describe tongue tissue from a cadaver of a 74-year-old woman. Using an indentation experiment the two parameters, $\mathrm{C}_{10}$ and $\mathrm{C}_{20}$, were respectively 192 and $90 \mathrm{~Pa}$. However, due to the effect of vascularization and tissue shrinkage after excision, ex-vivo experiments differ significantly from in-vivo experiments, which makes them less suitable for parameter identification [160-162]. One of the in-vivo experiments was conducted by Cheng et al. [159] using Magnetic Resonance Elastography (MRE) on 7 participants in which they found a mean Young modulus of $7743 \mathrm{~Pa}$. However, due to the supine position of the participants in this study, the tongue was forced against the palate, causing muscle activation and therefore resulting in an overestimation of the elasticity values. Moreover, in the setup proposed by Cheng et al. [159], the mechanical properties are retrieved by analyzing the displacement field induced by external $80 \mathrm{~Hz}$ vibrations which in combination with a mouthguard to keep the tongue in place, which can be uncomfortable for study subjects. The 
other in-vivo study by Schiavone et al. [158] used an aspiration method to acquire tissue properties - a technique that is comparable with commercial products for elasticity measurements of the skin such as the cutometer (Courage + Khazaka electronic $\mathrm{GmbH}$, Germany). In these techniques, a chamber with a circular aperture is depressurized to suck the tissue into the lumen. Using a camera, the height of the tissue iscalculated. Byusing the height of thetissue and thenegative pressurefor aFE inverse simulation of measurement, the tissue parameters of the Yeoh strain energy function can be determined. A new iteration of this technique is used in Chapter 5 to determine tongue elasticity both under general anesthesia and while awake.

\subsection{AIM AND OUTLINE}

This thesis aims to advance the development of biomechanical models of the tongue with the specific aim of predicting expected postoperative function loss. This is a multifaceted problem, as many variables influence the postoperative function of the tongue. This thesis begins with a proof of concept. Chapter 2 describes the first version of a virtual surgery tool to simulate a partial glossectomy and the resulting postoperative motion using a theoretical model of the tongue. By creating this tool, the imperative for a reliable model and the. Requirements of a clinically usable tool became more apparent. The following chapters dive deeper into a couple of these requirements, but also explore options that might be easier to implement. Chapter 3 focuses on improving the technique to measure tongue movement expressed in Range of Motion (ROM), elaborating on the optical tracking method first introduced by van Dijk et al. [71]. Chapter 4 describes the occurrence of five different tongue movements in a Dutch population-based observatory study with 400 participants, including whether or not participants are aware that they possess the ability to make a certain movement. The latter is an important aspect to consider when starting research on the predictive value of specific preoperative tongue movements. The virtual surgery tool, as described in Chapter 2, clarified the need for a method to measure the elasticity of the tongue and the elasticity of fibrous tissue. Chapter 5 addresses the technical aspects of a new aspiration-based method to measure the elasticity of tongue tissue. To validate this minimally invasive method, a study was initiated to measure the differences between the tongue elasticity while under general anesthesia and while awake. Chapter 6 addresses the need for more detailed and personalized information about the muscle architecture of the individual tongue, and 10 personalized biomechanical models were created based on novel diffusion-weighted imaging techniques developed by Voskuilen et al. [150]. Chapter 7 applies this same method for two pre- and post-op cases to demonstrate the concept of future postoperative tongue function prediction. Chapter 8 brings the thesis to a close with summaries and a final conclusion. 


\subsection{REFERENCES}

1. Kreeft AM, Tan IB, Leemans CR, Balm AJM. The surgical dilemma in advanced oral and oropharyngeal cancer: how we do it. Clin Otolaryngol. 2011;36: 260-266. doi:10.1111/ j.1749-4486.2011.02299.x

2. Kreeft AM, Van Der Molen L, Hilgers FJ, Balm AJ. Speech and swallowing after surgical treatment of advanced oral and oropharyngeal carcinoma: A systematic review of the literature. Eur Arch Oto-Rhino-Laryngology. Springer; 2009;266: 1687-1698. doi:10.1007/s00405-009-1089-2

3. Kreeft AM, Tan IB, van den Brekel MWM, Hilgers FJ, Balm AJM. The surgical dilemma of 'functional inoperability' in oral and oropharyngeal cancer: current consensus on operability with regard to functional results. Clin Otolaryngol. 2009;34: 140-146. doi:10.1111/j.1749-4486.2009.01884.x

4. Kreeft AM. Functional inoperability of oral and oropharyngeal cancer. University of Amsterdam. 2013.

5. Van Alphen MJA. Towards a predictive model for functional loss after oral cancer treatment. University of Twente; 2015. doi:10.3990/1.9789036539173

6. Eskes M. Surface electromyography in personalised modelling of the head and neck. University of Twente. 2017. doi:10.3990/1.9789036544474

7. A. Christopoulos, S. P. Moubayed, M. E. Nader, J. E. Ghannoum, T. Ayad A, Meyers AD. Mouth Anatomy [Internet]. 2015 [cited 15 May 2020]. Available: https://emedicine. medscape.com/article/1899122-overview

8. Kier WM, Smith KK. Tongues, tentacles and trunks: the biomechanics of movement in muscular-hydrostats. Zool J Linn Soc. 1985;83: 307-324. doi:10.1111/j.1096-3642.1985.tb01178.x

9. Agur A, Dalley A. Grant's Altas of Anatomy. 11th ed. Wolters Kluwer, Lippincott Williams \&Wilkins; 2005.

10. MooreKL,DalleyAF.Clinicallyorientedanatomy.6thed.LippincottWilliams\&Wilkins;2010.

11. Mu L, Sanders I. Human tongue neuroanatomy: Nerve supply and motor endplates. Clin Anat. 2010;23: 777-791. doi:10.1002/ca.21011

12. Sanders I, Mu L. A three-dimensional atlas of human tongue muscles. Anat Rec. 2013;296: 1102-1114. doi:10.1002/ar.22711

13. Van Alphen MJAJA, Eskes M, Smeele LEE, Balm AJMJM, van der Heijden F. In vivo intraoperative hypoglossal nerve stimulation for quantitative tongue motion analysis. Comput Methods Biomech Biomed Eng Imaging Vis. Taylor \& Francis; 2017;5: 409415. doi:10.1080/21681163.2015.1072056

14. KENHUB. Tongue [Internet]. [cited 31 Aug 2020]. Available: https://www.kenhub.com/ en/library/anatomy/tongue

15. Warnakulasuriya S, Greenspan JS. Epidemiology of Oral and Oropharyngeal Cancers BT. In: Warnakulasuriya S, Greenspan JS, editors. Textbook of Oral Cancer: Prevention, Diagnosis and Management. Cham: Springer International Publishing; 2020. pp. 5-21. doi:10.1007/978-3-030-32316-5_2

16. Bray F, Ferlay J, Soerjomataram I, Siegel RL, Torre LA, Jemal A. Global cancer statistics 2018: GLOBOCAN estimates of incidence and mortality worldwide for 36 cancers in 185 countries. CA Cancer J Clin. 2018; doi:10.3322/caac.21492 
17. Ferlay J, Colombet M, Soerjomataram I, Mathers C, Parkin DM, Piñeros M, et al. Estimating the global cancer incidence and mortality in 2018: GLOBOCAN sources and methods. Int J Cancer. 2019;144: 1941-1953. doi:10.1002/ijc.31937

18. Ferlay J, Soerjomataram I, Dikshit R, Eser S, Mathers C, Rebelo M, et al. Cancer incidence and mortality worldwide: Sources, methods and major patterns in GLOBOCAN 2012. Int J Cancer. 2015;136: E359-E386. doi:10.1002/ijc.29210

19. Intergraal kankercentrum Nederland. Dutch Cancer Figures [Internet]. 2020 [cited 29 Jan 2020]. Available: www.cijfersoverkanker.nl

20. Hashibe M. Risk Factors for Cancer of the Mouth: Tobacco, Betel Quid, and Alcohol. Textbook of Oral Cancer: Prevention, Diagnosis and Management. 2020. pp. 24-29.

21. Trafford AM, Parisi R, Kontopantelis E, Griffiths CEM, Ashcroft DM. Association of Psoriasis With the Risk of Developing or Dying of Cancer: A Systematic Review and Meta-analysis. JAMA Dermatology. 2019;155: 1390-1403. doi:10.1001/ jamadermatol.2019.3056

22. Warnakulasuriya S. Global epidemiology of oral and oropharyngeal cancer. Oral Oncol. Elsevier Ltd; 2009;45: 309-316. doi:10.1016/j.oraloncology.2008.06.002

23. Chen YK, Huang HC, Lin LM, Lin CC. Primary oral squamous cell carcinoma: An analysis of 703 cases in southern Taiwan. Oral Oncol. 1999;35: 173-179. doi:10.1016/S13688375(98)00101-8

24. Negri E, Boffetta P, Berthiller J, CastellsagueX, Curado MP, Maso LD, et al. Family history of cancer: Pooled analysis in the International Head and Neck Cancer Epidemiology consortium. Int J Cancer. 2009;124: 394-401. doi:10.1002/ijc.23848

25. Furquim CP, Pivovar A, Amenábar JM, Bonfim C, Torres-Pereira CC. Oral cancer in Fanconi anemia: Review of 121 cases. Critical Reviews in Oncology/Hematology. 2018. pp. 35-40. doi:10.1016/j.critrevonc.2018.02.013

26. Bongers V, Braakhuis BJM, Tobi H, Lubsen H, Snow GB. The relation between cancer incidence among relatives and the occurrence of multiple primary carcinomas following head and neck cancer. Cancer Epidemiol Biomarkers Prev. 1996;5: 595-598.

27. Lacko M, Braakhuis BJM, Sturgis EM, Boedeker CC, Suárez C, Rinaldo A, et al. Genetic susceptibility to head and neck squamous cell carcinoma. International Journal of Radiation Oncology Biology Physics. 2014.pp.38-48. doi:10.1016/j.jjrobp.2013.09.034

28. Moller H. Changing incidence of cancer of thetongue, oral cavity, and pharynxin Denmark. J Oral Pathol Med. 1989;18: 224-229. doi:10.1111/j.1600-0714.1989.tb00767.x

29. Gupta N, Gupta R, Acharya AK, Patthi B, Goud V, Reddy S, et al. Changing Trends in oral cancer - a global scenario. Nepal J Epidemiol. International Nepal Epidemiological Association; 2016;6: 613-619. doi:10.3126/nje.v6i4.17255

30. Tota JE, Anderson WF, Coffey C, Califano J, Cozen W, Ferris RL, et al. Rising incidence of oral tongue cancer among white men and women in the United States, 1973-2012. Oral Oncol. 2017;67: 146-152. doi:10.1016/j.oraloncology.2017.02.019

31. Chaturvedi AK, Anderson WF, Lortet-Tieulent J, Paula Curado M, Ferlay J, Franceschi S, et al. Worldwide trends in incidence rates for oral cavity and oropharyngeal cancers. J Clin Oncol. 2013;31: 4550-4559. doi:10.1200/JCO.2013.50.3870

32. Llewellyn CD, Johnson NW, Warnakulasuriya KAAS. Risk factors for squamous cell carcinoma of the oral cavity in young people - A comprehensive literature review. Oral Oncology. 2001. pp. 401-418. doi:10.1016/S1368-8375(00)00135-4 
33. Elwood JM, Youlden DR, Chelimo C, Ioannides SJ, Baade PD. Comparison of oropharyngeal and oral cavity squamous cell cancer incidence and trends in New Zealand and Queensland, Australia. Cancer Epidemiol. 2014;38: 16-21. doi:10.1016/j. canep.2013.12.004

34. Castellsagué X, Alemany L, Quer M, Halec G, Quirós B, Tous S, et al. HPV Involvement in Head and Neck Cancers: Comprehensive Assessment of Biomarkers in 3680 Patients. J Natl Cancer Inst. 2016; doi:10.1093/jnci/djv403

35. Hong AM, Dobbins TA, Lee CS, Jones D, Harnett GB, Armstrong BK, et al. Human papillomavirus predicts outcome in oropharyngeal cancer in patients treated primarily with surgery or radiation therapy. Br J Cancer. 2010;103: 1510-1517. doi:10.1038/ sj.bjc.6605944

36. Chaturvedi AK, Engels EA, Anderson WF, Gillison ML. Incidence trends for human papillomavirus-related and -unrelated oral squamous cell carcinomas in the United States. J Clin Oncol. 2008;26: 612-619. doi:10.1200/JCO.2007.14.1713

37. Ang KK, Harris J, Wheeler R, Weber R, Rosenthal DI, Nguyen-Tân PF, et al. Human Papillomavirus and Survival of Patients with Oropharyngeal Cancer. N Engl J Med. 2010;363: 24-35. doi:10.1056/NEJMoa0912217

38. Lai K, Killingsworth M, Matthews S, Caixeiro N, Evangelista C, Wu X, et al. Differences in survival outcome between oropharyngeal and oral cavity squamous cell carcinoma in relation to HPV status. J Oral Pathol Med. 2017;46: 574-582. doi:10.1111/jop.12535

39. Bagan J, Sarrion G, Jimenez Y. Oral cancer: Clinical features. Oral Oncol. 2010;46: 414417. doi:10.1016/j.oraloncology.2010.03.009

40. Walvekar RR, Chaukar DA, Deshpande MS, Pai PS, Chaturvedi P, Kakade AC, et al. Prognostic factors for loco-regional failure in early stage (I and II) squamous cell carcinoma of the gingivobuccal complex. Eur Arch Oto-Rhino-Laryngology. 2010;267: 1135-1140. doi:10.1007/s00405-009-1201-7

41. Silverman S, Gorsky M, Lozada F. Oral leukoplakia and malignant transformation. A follow-up study of 257 patients. Cancer. 1984;53: 563-568. doi:10.1002/10970142(19840201)53:3<563::AID-CNCR2820530332>3.0.CO;2-F

42. Warnakulasuriya S, Ariyawardana A. Malignant transformation of oral leukoplakia: A systematic review of observational studies. J Oral Pathol Med. 2016;45: 155-166. doi:10.1111/jop.12339

43. Bagan J V, Bagan-Debon L. Clinical Presentation and Differential Diagnosis of Oral Cancer BT - Textbook of Oral Cancer: Prevention, Diagnosis and Management. In: Warnakulasuriya S, Greenspan JS, editors. Cham: Springer International Publishing; 2020. pp. 47-54. doi:10.1007/978-3-030-32316-5_5

44. Lydiatt WM, Patel SG, O'Sullivan B, Brandwein MS, Ridge JA, Migliacci JC, et al. Head and Neck cancers-major changes in the American Joint Committee on cancer eighth edition cancer staging manual. CA Cancer J Clin. United States; 2017;67: 122-137. doi:10.3322/caac.21389

45. Murthy S, Low T-HH, Subramaniam N, Balasubramanian D, Sivakumaran V, Anand A, et al. Validation of the eighth edition AJCC staging system in early T1 to T2 oral squamous cell carcinoma. J Surg Oncol. United States; 2019;119: 449-454. doi:10.1002/jso.25348

46. Huang SH, O'Sullivan B. Overview of the 8th Edition TNM Classification for Head and Neck Cancer. Curr Treat Options Oncol. 2017;18:40. doi:10.1007/s11864-017-0484-y 
47. Datema FR, Ferrier MB, Vergouwe Y, Moya A, Molenaar J, Piccirillo JF, et al. Update and external validation of a head and neck cancer prognostic model. Head Neck. 2013;35: 1232-1237. doi:10.1002/hed.23117

48. Pfister DG, Spencer S, Brizel DM, Burtness B, Busse PM, Caudell JJ, et al. Clinical practice guidelines in oncology. Head Neck Cancers Version. 2019;1.

49. Copper MP, Tan IB, Oppelaar H, Ruevekamp MC, StewartFA. Meta-tetra(hydroxyphenyl) chlorin photodynamic therapy in early-stage squamous cell carcinoma of the head and neck. Arch Otolaryngol - Head Neck Surg. 2003;129: 709-711. doi:10.1001/ archotol.129.7.709

50. Van Der Molen L, Van Rossum MA, Burkhead LM, Smeele LE, Hilgers FJM. Functional outcomes and rehabilitation strategies in patients treated with chemoradiotherapy for advanced head and neck cancer: A systematic review. Eur Arch Oto-Rhino-Laryngology. 2009;266: 889-900. doi:10.1007/s00405-008-0817-3

51. Bauml JM, Aggarwal C, Cohen RB. Immunotherapy for head and neck cancer: where are we now and where are we going? Ann Transl Med. 2019;7: S75-S75. doi:10.21037/ atm.2019.03.58

52. Gettinger S, Horn L, Jackman D, Spigel D, Antonia S, Hellmann M, et al. Five-year follow-up of nivolumab in previously treated advanced non-small-cell lung cancer: Results from the CA209-003 study. J Clin Oncol. 2018;36: 1675-1684. doi:10.1200/ JCO.2017.77.0412

53. Ferris RL. Immunology and immunotherapy of head and neck cancer. Journal of Clinical Oncology. 2015. pp. 3293-3304. doi:10.1200/JCO.2015.61.1509

54. Funk GF, Karnell LH, Christensen AJ. Long-term health-related quality of life in survivors of head and neck cancer. Arch Otolaryngol - Head Neck Surg. 2012;138: 123-133. doi:10.1001/archoto.2011.234

55. Dwivedi RC, Kazi RA, Agrawal N, Nutting CM, Clarke PM, Kerawala CJ, et al. Evaluation of speech outcomes following treatment of oral and oropharyngeal cancers. Cancer Treatment Reviews Elsevier Ltd; 2009 pp. 417-424. doi:10.1016/j.ctrv.2009.04.013

56. Sun J, Weng Y, Li J, Wang G, Zhang Z. Analysis of Determinants on Speech Function After Glossectomy. J Oral Maxillofac Surg. 2007;65: 1944-1950. doi:10.1016/j. joms.2006.11.017

57. Bressmann T, Sader R, Whitehill TL, Samman N. Consonant intelligibility and tongue motility in patients with partial glossectomy. J Oral Maxillofac Surg. 2004;62: 298-303. doi:10.1016/j.joms.2003.04.017

58. Schache AG, Lieger O, Rogers P, Kelly A, Newman L, Kalavrezos N. Predictors of swallowing outcome in patients treated with surgery and radiotherapy for advanced oral and oropharyngeal cancer. Oral Oncol. 2009;45: 803-808. doi:10.1016/j. oraloncology.2008.12.010

59. Zuydam AC, Lowe D, Brown JS, Vaughan ED, Rogers SN. Predictors of speech and swallowing function following primary surgery for oral and oropharyngeal cancer. Clin Otolaryngol. 2005;30: 428-437. doi:10.1111/j.1365-2273.2005.01061.x

60. Langendijk JA, Doornaert P, Rietveld DHF, Verdonck-de Leeuw IM, René Leemans C, Slotman BJ. A predictive model for swallowing dysfunction after curative radiotherapy in head and neck cancer. Radiother Oncol. 2009;90: 189-195. doi:10.1016/j. radonc.2008.12.017 
61. Weber C, Dommerich S, Pau HW, Kramp B. Limited mouth opening after primary therapy of head and neck cancer. Oral Maxillofac Surg. 2010;14:169-173. doi:10.1007/ s10006-010-0220-2

62. Chuanjun C, Zhiyuan Z, Shaopu G, Xinquan J, Zhihong Z. Speech after partial glossectomy: A comparison between reconstruction and nonreconstruction patients. J Oral Maxillofac Surg. 2002;60: 404-407. doi:10.1053/joms.2002.31228

63. Amir Z, Wynn P, Chan F, Strauser D, Whitaker S, Luker K. Return to work after cancer in the UK: Attitudes and experiences of line managers. J Occup Rehabil. 2010;20: 435442. doi:10.1007/s10926-009-9197-9

64. Mehnert A. Employment and work-related issues in cancer survivors. Critical Reviews in Oncology/Hematology. 2011. pp. 109-130. doi:10.1016/j.critrevonc.2010.01.004

65. Duijts SFA, Van Egmond MP, Spelten E, Van Muijen P, Anema JR, Van Der Beek AJ. Physical and psychosocial problems in cancer survivors beyond return to work: A systematic review. Psychooncology. 2014;23: 481-492. doi:10.1002/pon.3467

66. Kuhl PK. Brain Mechanisms in Early Language Acquisition. Neuron. 2010. pp. 713-727. doi:10.1016/j.neuron.2010.08.038

67. Kimata Y, Sakuraba M, Hishinuma S, Ebihara S, Hayashi R, Asakage T, et al. Analysis of the relations between the shape of the reconstructed tongue and postoperative functions after subtotal or total glossectomy. Laryngoscope. 2003;113: 905-909. doi:10.1097/00005537-200305000-00024

68. Loro WA, Owens B. Unilateral hypoglossal nerve injury in a collegiate wrestler: A case report. J Athl Train. 2009;44: 534-537. doi:10.4085/1062-6050-44.5.534

69. Mosier K, Liu WC, Behin B, Lee C, Baredes S. Cortical adaptation following partial glossectomy with primary closure: Implications for reconstruction of the oral tongue. Ann Otol Rhinol Laryngol. 2005;114: 681-687. doi:10.1177/000348940511400905

70. Shin YS, Koh YW, Kim SH, Jeong JH, Ahn S, Hong HJ, et al. Radiotherapy deteriorates postoperative functional outcome after partial glossectomy with free flap reconstruction. J Oral Maxillofac Surg. 2012;70: 216-220. doi:10.1016/j.joms.2011.04.014

71. van Dijk S, van Alphen MJA, Jacobi I, Smeele LE, van der Heijden F, Balm AJM. A New Accurate 3D Measurement Tool to Assess the Range of Motion of the Tongue in Oral Cancer Patients: A Standardized Model. Dysphagia. Springer US; 2016;31: 97-103. doi:10.1007/s00455-015-9665-7

72. Ingervall B, Schmoker R. Effect of surgical reduction of the tongue on oral stereognosis, oral motor ability, and the rest position of the tongue and mandible. Am J Orthod Dentofac Orthop. 1990;97: 58-65. doi:10.1016/S0889-5406(05)81710-X

73. Suarez-Cunqueiro MM, Schramm A, Schoen R, Seoane-Lestón J, Otero-Cepeda XL, Bormann $\mathrm{KH}$, et al. Speech and swallowing impairment after treatment for oral and oropharyngeal cancer. Arch Otolaryngol - Head Neck Surg. 2008;134: 1299-1304. doi:10.1001/archotol.134.12.1299

74. Borggreven PA, Aaronson NK, Verdonck-de Leeuw IM, Muller MJ, Heiligers MLCH, Bree R de, et al. Quality of life after surgical treatment for oral and oropharyngeal cancer: A prospective longitudinal assessment of patients reconstructed by a microvascular flap. Oral Oncol. 2007;43: 1034-1042. doi:10.1016/j.oraloncology.2006.11.017

75. Rieger J, Dickson N, Lemire R, Bloom K, Wolfaardt J, Wolfaardt U, et al. Social perception of speech in individuals with oropharyngeal reconstruction. J Psychosoc Oncol. 2006;24: 33-51. doi:10.1300/J077v24n04_03 
76. Ward EC, van As-Brooks CJ. Head and neck cancer: treatment, rehabilitation, and outcomes. Plural Publishing; 2014.

77. Mittal BB, Pauloski BR, Haraf DJ, Pelzer HJ, Argiris A, Vokes EE, et al. Swallowing dysfunction - Preventative and rehabilitation strategies in patients with head-and-neck cancers treated with surgery, radiotherapy, and chemotherapy: A critical review. Int J Radiat Oncol Biol Phys. 2003;57: 1219-1230. doi:10.1016/S0360-3016(03)01454-8

78. Kotz T, Costello R, Li Y, Posner MR. Swallowing dysfunction after chemoradiation for advanced squamous cell carcinoma of the head and neck. Head Neck. 2004;26: $365-$ 372. doi:10.1002/hed.10385

79. KraaijengaSAC,MolenLvander,StuiverMM,TakesRP,Al-MamganiA,BrekelMWMva.den, etal.Efficacyofanovelswallowingexerciseprogramforchronicdysphagiainlong-termhead and neck cancer survivors. Head Neck. 2017;39: 1943-1961. doi:10.1002/hed.24710

80. Van den Steen L, Baudelet M, Tomassen P, Bonte K, De Bodt M, Van Nuffelen G. Effect of tongue-strengthening exercises on tongue strength and swallowing-related parameters in chronic radiation-associated dysphagia. Head Neck. 2020;42: 22982307. doi:10.1002/hed.26179

81. Bjordal K, Hammerlid E, Ahlner-Elmqvist M, De Graeff A, Boysen M, Evensen JF, et al. Quality of life in head and neck cancer patients: validation of the European Organization for Research and Treatment of Cancer Quality of Life Questionnaire-H\&N35. J Clin Oncol. American Society of Clinical Oncology; 1999;17: 1008.

82. Ojo B, Genden EM, Teng MS, Milbury K, Misiukiewicz KJ, Badr H. A systematic review of head and neck cancer quality of life assessment instruments. Oral Oncol. 2012;48: 923-937. doi:10.1016/j.oraloncology.2012.03.025

83. Weymuller EA, Alsarraf R, Yueh B, Deleyiannis FWB, Coltrera MD. Analysis of the performance characteristics of the University of Washington Quality of Life instrument and its modification (UW-QOL-R). Arch Otolaryngol - Head Neck Surg. American Medical Association; 2001;127: 489-493. doi:10.1001/archotol.127.5.489

84. List MA, D'Antonio LL, Cella DF, Siston A, Mumby P, Haraf D, et al. The performance status scale for head and neck cancer patients and the functional assessment of cancer therapy-head and neck scale: A study of utility and validity. Cancer. Wiley Online Library; 1996;77: 2294-2301. doi:10.1002/(SICI)1097-0142(19960601)77:11<2294::AIDCNCR17>3.0.CO;2-S

85. Terrell JE, Nanavati KA, Esclamado RM, Bishop JK, Bradford CR, Wolf GT. Head and neck cancer-specific quality of life: Instrument validation. Arch Otolaryngol - Head Neck Surg. American Medical Association; 1997;123: 1125-1132. doi:10.1001/ archotol.1997.01900100101014

86. Mlynarek AM, Rieger JM, Harris JR, O'Connell DA, Al-Qahtani KH, Ansari K, et al. Methods of functional outcomes assessment following treatment of oral and oropharyngeal cancer: Review of the literature. J Otolaryngol - Head Neck Surg. 2008;37: 2-10. doi:10.2310/7070.2008.1001

87. Husaini H, Krisciunas GP, Langmore S, Mojica JK, Urken ML, Jacobson AS, et al. A survey of variables used by speech-language pathologists to assess function and predict functional recovery in oral cancer patients. Dysphagia. 2014;29:376-386. doi:10.1007/ s00455-014-9520-2

88. Konstantinović VS, Dimić ND. Articulatory function and tongue mobility after surgery followed by radiotherapy for tongue and floor of the mouth cancer patients. Br J Plast Surg. 1998;51: 589-593. doi:10.1054/bjps.1998.0202 
89. Matsui Y, Ohno K, Yamashita Y, Takahashi K. Factors influencing postoperative speech function of tongue cancer patients following reconstruction with fasciocutaneous/ myocutaneous flaps-a multicenter study. Int J Oral Maxillofac Surg. 2007;36: 601609. doi:10.1016/j.ijom.2007.01.014

90. Logemann JA. Role of the modified barium swallow in management of patients with dysphagia. Otolaryngology - Head and Neck Surgery. 1997. pp. 335-338. doi:10.1016/ S0194-5998(97)70269-9

91. Parthasarathy $V$, Prince JL, Stone M, Murano EZ, NessAiver M. Measuring tongue motion from tagged cine-MRI using harmonic phase (HARP) processing. J Acoust Soc Am. 2007; doi:10.1121/1.2363926

92. Lazarus CL, Logemann JA, Pauloski BR, Rademaker AW, Larson CR, Mittal BB, et al. Swallowing and Tongue Function Following Treatment for Oral and Oropharyngeal Cancer. J Speech, Lang Hear Res. 2000;43: 1011-1023. doi:10.1044/js/hr.4304.1011

93. Kreeft AM, Rasch CRN, Muller SH, Pameijer FA, Hallo E, Balm AJM. Cine MRI of swallowing in patients with advanced oral or oropharyngeal carcinoma: A feasibility study. Eur Arch Oto-Rhino-Laryngology. 2012;269: 1703-1711. doi:10.1007/s00405011-1861-y

94. Perkell JS, Cohen MH, Svirsky M a, Matthies ML, Garabieta I, Jackson MTT. Electromagnetic midsagittal articulometer systems for transducing speech articulatory movements. J Acoust Soc Am. 1992;92: 3078-3096. doi:10.1121/1.404204

95. Engwall O. Combining MRI, EMA and EPG measurements in a three-dimensional tongue model. Speech Commun. 2003;41: 303-329. doi:10.1016/S0167-6393(02)00132-2

96. Kroos C. Evaluation of the measurement precision in three-dimensional Electromagnetic Articulography (Carstens AG500). J Phon. 2012;40: 453-465. doi:10.1016/j. wocn.2012.03.002

97. Echternach M, Burk F, Burdumy M, Traser L, Richter B. Morphometric differences of vocal tract articulators in different loudness conditions in singing. PLoS One. 2016;11. doi:10.1371/journal.pone.0153792

98. Lim Y, Zhu Y, Lingala SG, Byrd D, Narayanan S, Nayak KS. 3D dynamic MRI of the vocal tract during natural speech. Magn Reson Med. 2019;81: 1511-1520. doi:10.1002/ mrm. 27570

99. Eskes M, Van Alphen MJA, Balm AJM, Smeele LE, Brandsma D, Van Der Heijden F. Predicting 3D lip shapes using facial surface EMG. PLoS One. 2017;12. doi:10.1371/ journal.pone.0175025

100. O'Connor CM, Lowery MM, Doherty LS, McHugh M, O'Muircheartaigh C, Cullen J, et al. Improved surface EMG electrode for measuring genioglossus muscle activity. Respir Physiol Neurobiol. 2007;159: 55-67. doi:10.1016/j.resp.2007.05.011

101. Blumen MB, Perez De La Sota A, Quera-Salva MA, Frachet B, Chabolle F, Lofaso F. Tongue mechanical characteristics and genioglossus muscle EMG in obstructive sleep apnoea patients. Respir Physiol Neurobiol. 2004;140: 155-164. doi:10.1016/j. resp.2003.12.001

102. Doble EA, Leiter JC, Knuth SL, Daubenspeck JA, Bartlett D. A noninvasive intraoral electromyographic electrode for genioglossus muscle. J Appl Physiol. 1985;58: 13781382. doi:10.1152/jappl.1985.58.4.1378

103. Staveren ES. Towards a personlalised biomechanical model - sEMG measurements on the tongue and motor unit identification [Internet]. University of Twente. 2017. Available: http://purl.utwente.nl/essays/74078 
104. Courant R. Variational methods for the solution of problems of equilibrium and vibrations. Bulletin of the American Mathematical Society. 1943. doi:10.1090/S00029904-1943-07818-4

105. Hrennikoff A. Solution of Problems of Elasticity by the Frame-Work Method. Appl Sci Res. 1941;A8: 169-175.

106. Clough RW. The Finite Element Method in Plane Stress Analysis. Proceedings of the 2nd Conference on Electronic Computation of American Society of Civil Engineers. 1960. pp. 345-378.

107. Rao JS. Finite Element Methods. History of Mechanism and Machine Science. Springer Dordrecht; 2011. pp. 141-183. doi:10.1007/978-94-007-1165-5_13

108. Lloyd JE, Stavness I, Fels S. ArtiSynth: A Fast Interactive Biomechanical Modeling Toolkit Combining Multibody and Finite Element Simulation. Studies in Mechanobiology, Tissue Engineering and Biomaterials. 2012. pp. 355-394. doi:10.1007/8415_2012_126

109. Anand B, Kumar D, Aeran H, Anand V, Gulati M, Bahuguna R. Evaluation of stress patterns in bone around dental implant for different abutment angulations under axial and oblique loading: A finite element analysis. Natl J Maxillofac Surg. Wolters Kluwer-Medknow Publications; 2013;4: 46. doi:10.4103/0975-5950.117882

110. Li J, Zhao Z, Yin P, Zhang L, Tang P. Comparison of three different internal fixation implants in treatment of femoral neck fracture - A finite element analysis. J Orthop Surg Res. 2019;14. doi:10.1186/s13018-019-1097-x

111. Taylor CA, Hughes TJR, Zarins CK. Finite element modeling of blood flow in arteries. Comput Methods Appl Mech Eng. 1998;158: 155-196. doi:10.1016/S00457825(98)80008-X

112. Boyle PM, Zghaib T, Zahid S, Ali RL, Deng D, Franceschi WH, et al. Computationally guided personalized targeted ablation of persistent atrial fibrillation. Nat Biomed Eng. 2019;3: 870-879. doi:10.1038/s41551-019-0437-9

113. Van Alphen MJA, Kreeft AM, Van Der Heijden F, Smeele LE, Balm AJM. Towards virtual surgery in oral cancer to predict postoperative oral functions preoperatively. Br J Oral Maxillofac Surg. British Association of Oral and Maxillofacial Surgeons; 2013;51: 747 751. doi:10.1016/j.bjoms.2013.06.012

114. Buchaillard S, Perrier P, Payan Y. A biomechanical model of cardinal vowel production: muscle activations and the impact of gravity on tongue positioning. J Acoust Soc Am. 2009;126: 2033-2051. doi:10.1121/1.3204306

115. Gérard J-M, Perrier P, Payan Y. 3D biomechanical tongue modeling to study speech production. Speech Prod Model Phonetic Process Tech. 2006; 85-102. Available: http://hal.archives-ouvertes.fr/hal-00108521/

116. Gerard J-M, Wilhelms-Tricarico R, Perrier P, Payan Y, Gérard J-M, Wilhelms-Tricarico R, et al. A 3D dynamical biomechanical tongue model to study speech motor control. Res Dev Biomech. 2003;1: 49-64.

117. Perkell JS. A physiologically-oriented model of tongue activity in speech production. [Internet]. Massachusetts Institute of Technology. 1974. Available: http://hdl.handle. net/1721.1/29190

118. Perrier P, Payan Y, Nazari MA, Hermant N, Rohan P-Y, Lobos C, et al. Speech biomechanics: What have we learned and modeled since Joseph Perkell's tongue model In 1974? J Acoust Soc Am. 2016;139: 2193-2193. doi:10.1121/1.4950528 
119. Payan Y, Bettega G, Raphaël B. A biomechanical model of the human tongue and its clinical implications. Lecture Notes in Computer Science (including subseries Lecture Notes in Artificial Intelligence and Lecture Notes in Bioinformatics). 1998. pp. 688-695. doi:10.1007/bfb0056255

120. Kiritani S, Miyawaki K, Fujimura O, Miller JE. Computational model of the tongue. New Jersey, Hongo Tokyo; 1975.

121. Kakita Y, Fujimura O. Computational model of the tongue: A revised version. J Acoust Soc Am. 1977;62: S15-S16. doi:10.1121/1.2016043

122. Wilhelms-Tricarico R. Physiological modeling of speech production: methods for modeling soft-tissue articulators. J Acoust Soc Am. United States, United States; 1995;97: 3085-3098. doi:10.1121/1.411871

123. Honda K. Organization of tongue articulation for vowels. J Phon. 1996;24: 39-52. doi:10.1006/jpho.1996.0004

124. Payan $Y$, Perrier P. Synthesis of $V-V$ sequences with a 2D biomechanical tongue model controlled by the equilibrium point hypothesis 1. Speech Commun. 1997;22: 185-205. doi:10.1016/S0167-6393(97)00019-8

125. Gerard JMM, Ohayon J, Luboz V, Perrier P, Payan Y. Non-linear elastic properties of the lingual and facial tissues assessed by indentation technique: Application to the biomechanics of speech production. Med Eng Phys. Elsevier; 2005;27: 884-892. doi:10.1016/j.medengphy.2005.08.001

126. Wilhelms-Tricarico R. Developmentofatongueandmouthfloormodelfornormalizationand biomechanical modelling. Proceedings of the Fifth Speech Production Seminar and CREST Workshop on Models of Speech Production. Kloster Seeon, Bavaria; 2000. pp. 141-148.

127. Huang Y, White DP, Malhotra A. Use of Computational Modeling to Predict Responses to Upper Airway Surgery in Obstructive Sleep Apnea. Laryngoscope. 2007;117: 648653. doi:10.1097/MLG.0b013e318030ca55

128. Vogt F, Lloyd JE, Buchaillard S, Perrier P, Chabanas M, Payan Y, et al. Efficient 3D finite element modeling of a muscle-activated tongue. Lect Notes Comput Sci. 2006; 19-28. doi:10.1007/11790273_3

129. Buchaillard S, Brix M, Perrier P, Payan Y. Simulations of the consequences of tongue surgery on tongue mobility: Implications for speech production in post-surgery conditions. Int J Med Robot ComputAssist Surg. 2007;3: 252-261. doi:10.1002/rcs.142

130. Rohan P-Y, Lobos C, Nazari M a., Perrier P, Payan Y. Finite element models of the human tongue: a mixed-element mesh approach. Comput Methods Biomech Biomed Eng Imaging Vis. 2016;00: 1-11. doi:10.1080/21681163.2015.1105760

131. Hermant N, Perrier P, Payan Y. Human Tongue Biomechanical Modeling. In: Payan Y, Ohayon JBT-B of LO, editors. Biomechanics of Living Organs: Hyperelastic Constitutive Laws for Finite Element Modeling. Oxford: Academic Press; 2017. pp. 395-411. doi:10.1016/B978-0-12-804009-6.00019-5

132. Fujita S, Dang J, Suzuki N, Honda K. A Computational Tongue Model and its Clinical Application. Oral Sci Int. 2007;4: 97-109. doi:10.1016/S1348-8643(07)80004-8

133. Couteau B, Payan Y, Lavallée S. The mesh-matching algorithm: an automatic 3D mesh generator for finite element structures. J Biomech. 2000;33: 1005-1009. doi:10.1016/ S0021-9290(00)00055-5

134. Sigal IA, Hardisty MR, Whyne CM. Mesh-morphing algorithms for specimenspecific finite element modeling. J Biomech. 2008;41: 1381-1389. doi:10.1016/j. jbiomech.2008.02.019 
135. Fernandez JW, Mithraratne P, Thrupp SF, Tawhai MH, Hunter PJ. Anatomically based geometric modelling of the musculo-skeletal system and other organs. Biomech Model Mechanobiol. 2004;2: 139-155. doi:10.1007/s10237-003-0036-1

136. Grassi L, Hraiech N, Schileo E, Ansaloni M, Rochette M, Viceconti M. Evaluation of the generality and accuracy of a new mesh morphing procedure for the human femur. Med Eng Phys. 2011;33: 112-120. doi:10.1016/j.medengphy.2010.09.014

137. Bucki M, Lobos C, Payan Y. A fast and robust patient specific Finite Element mesh registration technique: Application to 60 clinical cases. Med Image Anal. Elsevier B.V.; 2010;14: 303-317. doi:10.1016/j.media.2010.02.003

138. Bijar A, Rohan P-Y, Perrier P, Payan Y. Atlas-Based Automatic Generation of SubjectSpecific Finite Element Tongue Meshes. Ann Biomed Eng. 2016;44:16-34. doi:10.1007/ s10439-015-1497-y

139. Barber DC, Oubel E, Frangi AF, Hose DR. Efficient computational fluid dynamics mesh generation by image registration. Med Image Anal. 2007;11: 648-662. doi:10.1016/j. media.2007.06.011

140. Nesme M, Kry PG, Jeřábková L, Faure F. Preserving topology and elasticity for embedded deformable models. ACM Trans Graph. 2009;28: 1-9. doi:10.1145/1531326.1531358

141. Sánchez CA, Li Z, Hannam AG, Abolmaesumi P, Agur A, Fels S. Constructing Detailed Subject-Specific Models of the Human Masseter. Lecture Notes in Computer Science. Springer; 2017. pp. 52-60. doi:10.1007/978-3-319-67552-7_7

142. Cleveland GG, Chang DC, Hazlewood CF, Rorschach HE. Nuclear magnetic resonance measurement of skeletal muscle: anisotrophy of the diffusion coefficient of the intracellular water. Biophys J. 1976;16: 1043-1053. doi:10.1016/S00063495(76)85754-2

143. Heemskerk AM, Sinha TK, Wilson KJ, Ding Z, Damon BM. Repeatability of DTIbased skeletal muscle fiber tracking. NMR Biomed. 2010;23: 294-303. doi:10.1002/ nbm.1463

144. Takenaka S, Yoshinaga T, Nozaki K, li S, Wada S. A mechanical deformation model of the tongue during speech production considering personalized anatomical structure. J Acoust Soc Am. 2018;144: 1907-1907. doi:10.1121/1.5068361

145. Mijailovich SM, Stojanovic B, Kojic M, Liang A, Wedeen VJ, Gilbert RJ. Derivation of a finite-element model of lingual deformation during swallowing from the mechanics of mesoscale myofiber tracts obtained by MRI. J Appl Physiol. 2010/08/05. American Physiological Society; 2010;109: 1500-1514. doi:10.1152/japplphysiol.00493.2010

146. Gaige TA, Benner T,Wang R, Wedeen VJ, Gilbert RJ. Three dimensional myoarchitecture of the human tongue determined in vivo by diffusion tensor imaging with tractography. J Magn Reson Imaging. 2007;26: 654-661. doi:10.1002/jmri.21022

147. Murano EZ, Shinagawa H, Zhuo J, Gullapalli RP, Ord RA, Prince JL, et al. Application of diffusion tensor imaging after glossectomy. Otolaryngol - Head Neck Surg. 2010;143: 304-306. doi:10.1016/j.otohns.2010.03.012

148. Shinagawa H, Murano EZ, Zhuo J, Gullapalli RP, Landman B, Prince JL, et al. Tongue muscle fiber tracking during tongue protrusion and rest. J Acoust Soc Am. 2006;120: 3354-3354. doi:10.1121/1.4781440

149. Tournier J-D, Calamante F, Connelly A. Robust determination of the fibre orientation distribution in diffusion MRI: non-negativity constrained super-resolved spherical deconvolution. Neuroimage. 2007;35: 1459-1472. doi:10.1016/j. neuroimage.2007.02.016 
150. Voskuilen L, Mazzoli V, Oudeman J, Balm AJM, van der Heijden F, Froeling M, et al. Crossing muscle fibers of the human tongue resolved in vivo using constrained spherical deconvolution. J Magn Reson Imaging. John Wiley \& Sons, Ltd; 2019;50: 96-105. doi:10.1002/jmri.26609

151. Voskuilen L, Smeele LE, Balm AJM, Heijden F Van Der, Strijkers GJ, Nederveen AJ. Generation of a muscle fibre orientation atlas of the in vivo tongue. Proc Intl Soc Mag Reson Med. Paris; 2018. p. 26:5217.

152. Wolfe G, Gasper E, Stoke J, Kretchman J, Anderson D, Czuba N, et al. College Physics for APß Courses. In: OpenStax [Internet]. 2015 [cited 17 Sep 2020]. Available: https:// openstax.org/books/college-physics-ap-courses/pages/5-3-elasticity-stress-and-strain

153. Fung YC. Biomechanics: Mechanical properties of living tissue. Springer-Verlag; 1993.

154. BuchaillardS, BrixM,PerrierP, PayanY.Useofabiomechanical tonguemodel to predictthe impact of tongue surgery on speech production. 5th International Workshop on Models and Analysis of Vocal Emissions for Biomedical Applications, MAVEBA 2007. 2007.

155. Stavness I, Lloyd JE, Fels S. Automatic prediction of tongue muscle activations using a finite element model. J Biomech. Elsevier; 2012;45: 2841-2848. doi:10.1016/j. jbiomech.2012.08.031

156. Wang YK, Nash MP, Pullan AJ, Kieser JA, Rohrle O. Model-based identification of motion sensor placement for tracking retraction and elongation of the tongue. Biomech Model Mechanobiol. Germany, Germany;2013;12:383-399. doi:10.1007/s10237-012-0407-6

157. Marckmann G, Verron E. Comparison of hyperelastic models for rubber-like materials. Rubber Chem Technol. 2006;79: 835-858. doi:10.5254/1.3547969

158. Schiavone P, Boudou T, Promayon E, Perrier P, Payan Y. A light sterilizable pipette device for the in vivo estimation of human soft tissues constitutive laws. 2008 30th Annual International Conference of the IEEE Engineering in Medicine and Biology Society. IEEE; 2008. pp. 4298-4301. doi:10.1109/IEMBS.2008.4650160

159. Cheng S, Gandevia SC, Green M, Sinkus R, Bilston LE. Viscoelastic properties of the tongue and soft palate using MR elastography. J Biomech. Elsevier; 2011;44: 450-454. doi:10.1016/j.jbiomech.2010.09.027

160. Kerdok AE, Ottensmeyer MP, Howe RD. Effects of perfusion on the viscoelastic characteristics of liver. J Biomech. United States, United States; 2006;39: 2221-2231. doi:10.1016/j.jbiomech.2005.07.005

161. Ottensmeyer MP. Invivo measurement of solid organ viscoelastic properties. Stud Health Technol Inform. Netherlands, Netherlands; 2002;85: 328-333. doi:10.3233/978-160750-929-5-328

162. Gefen A, Margulies SS. Are in vivo and in situ brain tissues mechanically similar? J Biomech. United States, United States; 2004;37: 1339-1352. doi:10.1016/j. jbiomech.2003.12.032 



\title{
AN INTERACTIVE SURGICAL SIMULATION TOOL TO ASSESS THE CONSEQUENCES OF A PARTIAL GLOSSECTOMY ON A BIOMECHANICAL MODEL OF THE TONGUE
}

\author{
K.D.R. Kappert \\ M.J.A. van Alphen \\ S. van Dijk \\ L.E. Smeele \\ A.J.M. Balm \\ F. van der Heijden
}

This chapter was published in Computer Methods in Biomechanics and Biomedical Engineering:

Kappert KDR, van Alphen MJA, van Dijk S, Smeele LE, Balm AJM, van der Heijden F. An interactive surgical simulation tool to assess the consequences of a partial glossectomy on a biomechanical model of the tongue. Comput Methods Biomech Biomed Engin. 2019;22: 827-839. doi:10.1080/10255842.2019.1599362

This work was presented at:

- NWHHT congress (Netherlands Working Group on Head and

Neck Cancer), Utrecht, 2017

- MIRA day, Enschede, 2016

- Joint Autumn Meeting of the Dutch and Danish Associations,

Scheveningen, 2017

- PWHHT, Amersfoort, 2018

- 15th International Symposium on Computer Methods in

Biomechanics and Biomedical Engineering and the 3th

Conference on Imaging and Visualization, Lisbon, 2018 


\section{ABSTRACT}

Oral cancer surgery has a negative influence on the quality of life (QOL). As a result of the complex physiology involved in oral functions, estimation of surgical effects on functionality remains difficult. We present a user-friendly biomechanical simulation of tongue surgery, including closure with suturing and scar formation, followed by an automated adaptation of a finite element (FE) model to the shape of the tongue. Different configurations of our FE model were evaluated and compared to a well-established FE model. We showed that the post-operative impairment as predicted by our model was qualitatively comparable to a patient case for five different tongue maneuvers. 


\subsection{INTRODUCTION}

Head and neck cancer is the sixth most common cancer in the world [1] and the worldwide incidence of lip and oral-cavity cancers is estimated at $2.1 \%$ [2]. In Western countries, oral cancers most frequently occur at the lateral border of the tongue, accounting for $40-50 \%$ of all the oral cancers [1,3]. Dependent on the local extension of the tongue cancer, surgery might interfere with vital functions such as swallowing, mastication and speech having a negative influence on the Quality of Life (QoL) [4,5]. When the lesion is accessible trans-orally, curative surgery is often the preferred treatment, provided that the post-operative functional loss is acceptable. If not, organ-sparing treatment options such as radiotherapy or combined chemotherapy and radiotherapy can be considered [6,7]. In considering these options, shared decision making and evidence-based patient counseling are important. This requires objective and accurate pre-operative information regarding the oncologic and functional outcome, and information provision in an understandable and preferably visual way [8,9]. However, due to the complex structures and systems involved in oral functions, it is often not possible for a treating physician to predict the functional consequences of any particular treatment by experience and reasoning alone [10]. Simulations using physiological computer models can help the treating physician to understand the effects of interventions in the tongue and to provide the patient with visual information to understand the functional consequences. In the past decade, progresses have been made in biomechanical modeling of the tongue: In 2006, Gérard et al. [11] created a Finite Element (FE) model based on (1) MRI data from a specific human subject; (2) accurate information about muscle anatomy from the Visible Human Project [12], and (3) the work of Wilhelms-Tricarico [13] for speech production modeling. Vogt et al. [14] and Buchaillard et al. [15,16] elaborated on this model to show the opportunities for the simulation of speech production, and also the simulation of surgery and reconstruction using a free flap approach. Hermant et al. [17] adapted and improved this model through the work of Rohan et al. [18] in creating a tongue model based on a manual segmentation of MRI and CT images. Fujita et al. [19] created a personalized tongue model specifically for the simulation of a partial glossectomy. Van Alphen et al. [20] created an FE model to show the effects of impairments on the movement of the tongue. These previous studies yielded promising results for use of FE models to predict the functional loss. In several of those studies, surgery was simulated by altering the stiffness of particular elements. However, this approach is not sufficient to simulate primary closure using sutures, which is the most commonly applied technique in stage T1T2 tumors [21].

Our aim was to develop a user-friendly tool to simulate the effects of a partial resection of the tongue using an FE model. In this study we address five questions: 
(a) Can we create an interactive and editable model while still performing comparably to other models? (b) Can we simulate and define a resection in the FE model? (c) Can we simulate primary closure of a resection by suturing? (d) Can we simulate scar tissue by a fibrotic volume? and (e) Can we simulate a patient case using this model?

\subsection{METHODS AND MATERIALS}

\section{Materials}

The shape and muscle structure of our tongue model were based on the FE model developed by Buchaillard et al. [15], which originates from the models of Gérard et al. [11,22]. Their model was converted by Stavness et al. [23] to work in ArtiSynth, an open-source Java-based platform for combined multibody and FE model simulation [24]. This software also includes an inverse simulation controller as described in Stavness et al. [23]. Our surgery tool was also created within the ArtiSynth environment while MeshLab was used for manually editing a mesh [25].

To demonstrate the abilities of the tool, the position of the apex of the tongue of a patient with a T1 (i.e. diameter $<2 \mathrm{~cm}$ ) tongue cancer has been recorded both preand post-operatively using three Aviator A1000-100gc (Basler) video cameras.

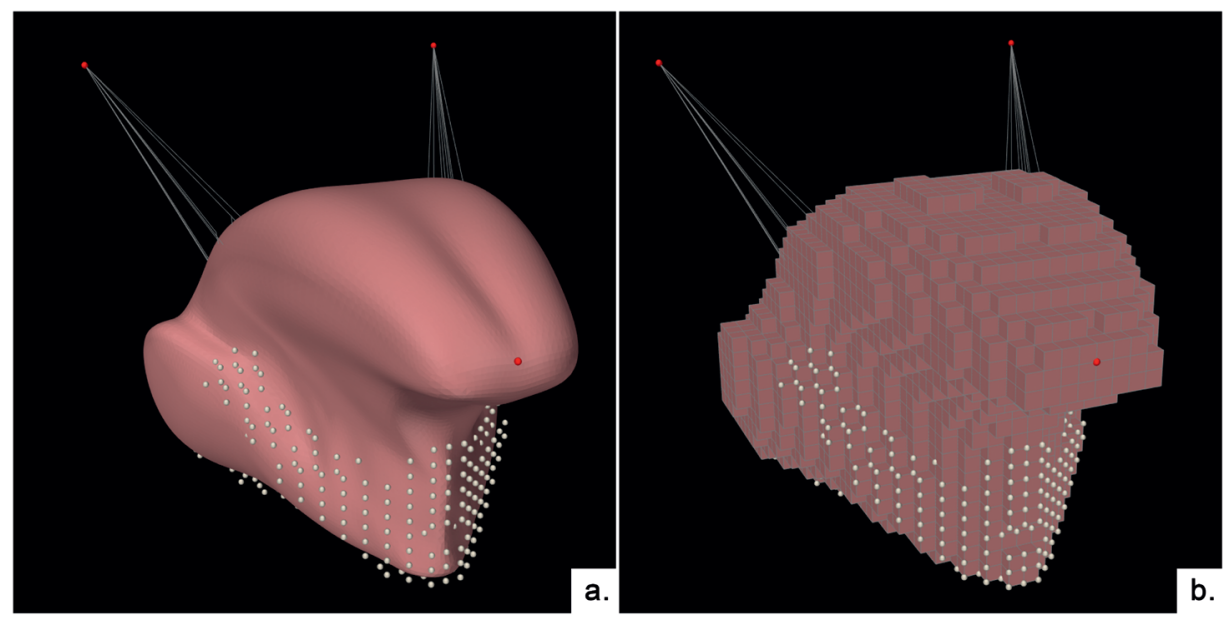

Figure 2.1 - a: The surface mesh of the model. b: The cubic-shaped FE model. The white dots are the non-dynamic nodes representing the attachment to the jaw.

\section{The model}

The biomechanics of a tongue were simulated using a 3D FE model. Using a least square subdivision surface algorithm from Boyé et al. [26], a smoothed version of the surface mesh from the FE model of Buchaillard et al. [15] was created in MeshLab [25]. An FE mesh consisting solely of cubic elements was generated to 
match the shape of the surface mesh so that the centroids of the elements were located within the surface mesh (Figure 2.1a). For visualization of the modeled dynamics, the high-resolution surface mesh was embedded into the cubic FE mesh (Figure 2.1b). Attachments to the jaw and hyoid bone were simulated by making the FE nodes in these areas non-dynamic.

This simplified representation enables quick generation of a new FE mesh when changes to the surface mesh are made. Quick generation is essential for surgical simulation (see section "Virtual surgery"), which is done at least two times during the procedure. Moreover, this technique enables us to easily generate new FE meshes for other (personalized) surface meshes in the future.

A small element size leads to a better representation of the model and the surgical incision, at the cost of longer computation time. The smallest element size with acceptable computation time was $16 \mathrm{~mm}^{3}$. This size was used in all the simulations. We also investigated the effect on mobility when increasing the element size to $39 \mathrm{~mm}^{3}$ and $141 \mathrm{~mm}^{3}$.

\section{Mechanical properties}

In order to compare our new editable model to the model of Buchaillard et al. [15] and Stavness et al. [23], we used the same mechanical properties. In these studies, the complex viscoelastic nature of tongue tissue was approached by a hyperelastic material. Element-based incompressibility ensures that the volume in each element stays the same throughout the simulation [27]. The material consisted of a 5-parameter Moony Rivlin material with $\mathrm{c}_{10}$ and $\mathrm{C}_{20}$ equal to 1037 and 486, respectively, and with $c_{01}, c_{11}$, and $c_{02}$ all being zero. A Rayleigh damping coefficients of $\alpha=40 \mathrm{~s}^{-1}$ and $\beta=0.03$ and a density of $1040 \mathrm{~kg} / \mathrm{m}^{3}$ were used. These parameters are based on ex-vivo research conducted by Gerard et al. [11] and were later fine-tuned by Buchaillard et al. [15]. An overview of other constitutive models to estimate the mechanical properties of the tongue can be found in Hermant et al. [17].

\section{Muscle properties and implementation}

The musculature was modeled using ArtiSynth's "muscle material" which effect is applied in addition to the regular material for an element. When excitation is applied to a muscle material, it generates an externally applied stress in the direction associated with the muscle [24]. For the simulation of the muscle stressstrain function, we used ArtiSynth's implementation of the method described by Blemker et al. [28]. In this method, stress and strain are influenced by muscle activation based on the direction of the particular muscle. In order to compare our model to the Buchaillard et al. [15] model, muscle divisions and muscle directions were converted and incorporated in our model. Using the Inverse 
Distance Weighted (IDW) interpolation, the string-based muscles of Buchaillard et al. [15] were converted into dense vector fields defining muscle directions and locations. The vector closest to the centroid of a particular element will determine the direction of its contraction upon activation. A typical distribution of element-muscles in an unedited model is demonstrated in Figure 2.2. This muscle representation also enables us to easily use other muscle configurations for future (personalized) models. Because of the long and compact trajectory of the styloglossus muscle towards the styloid process, we were unable to create a stable (automatically generated) element-based muscle for it. The styloglossus was, therefore, the only muscle simulated using string-based muscles.

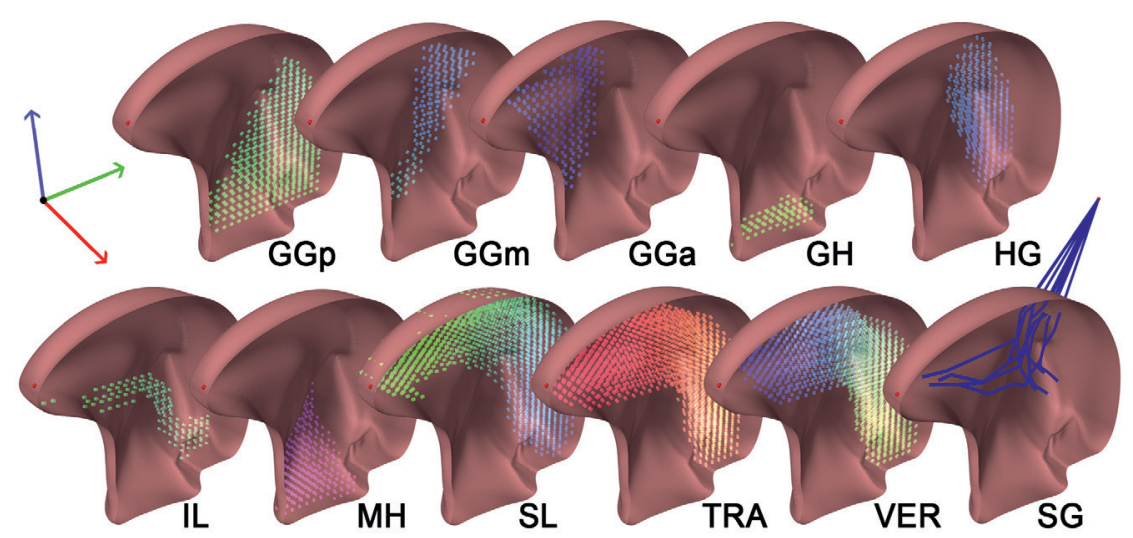

Figure 2.2 - Muscle bundles and directions. Every color-coded cylinder, located at the centroid of an element, represents the direction of contraction of that element. From left to right: genioglossus, -posterior (GGp), -middle (GGm), -anterior (GGa), geniohyoid muscle (GH), hyoglossus (HG), inferior longitudinal muscle (IL), mylohyoid muscle (MH), superior longitudinal muscle (SL), transverse muscle (TRA), vertical muscle (VER). The styloglossus (SG) is modeled as a fiber.

\section{Virtual surgery}

In the next paragraphs, a method is described to interactively create a postsurgical model. This method consists of three main steps:

Interactively defining a resection volume: The volume to be resected was delineated on the surface mesh of a tongue and subsequently subtracted from that mesh.

Virtual suturing: The remaining surface mesh and muscle vector fields were embedded in a cubic FE mesh that was generated to match the shape of the surface mesh. During the suturing procedure, the FE mesh facilitated the deformation of the surface mesh and the embedded muscle vector fields. 
Post-operative model: The hole in the deformed surface mesh was closed and the new FE mesh was generated. The muscle vector fields were converted to element-muscles.

\section{Interactively defining a resection volume}

The location of the surgical resection was delineated interactively by selecting a set of 3D points $\left(\mathbf{c}_{\mathbf{i}}\right)$ on the surface mesh. Using the chain of 3D points, two polygonal cones covering the area above and below these points were defined to form a closed 3D structure (Figure 2.3). Subsequently, all the faces enclosed by this structure were marked as "selected area". This method performed well for selecting the area's convex and concave surfaces, which are present on the tongue surface mesh.
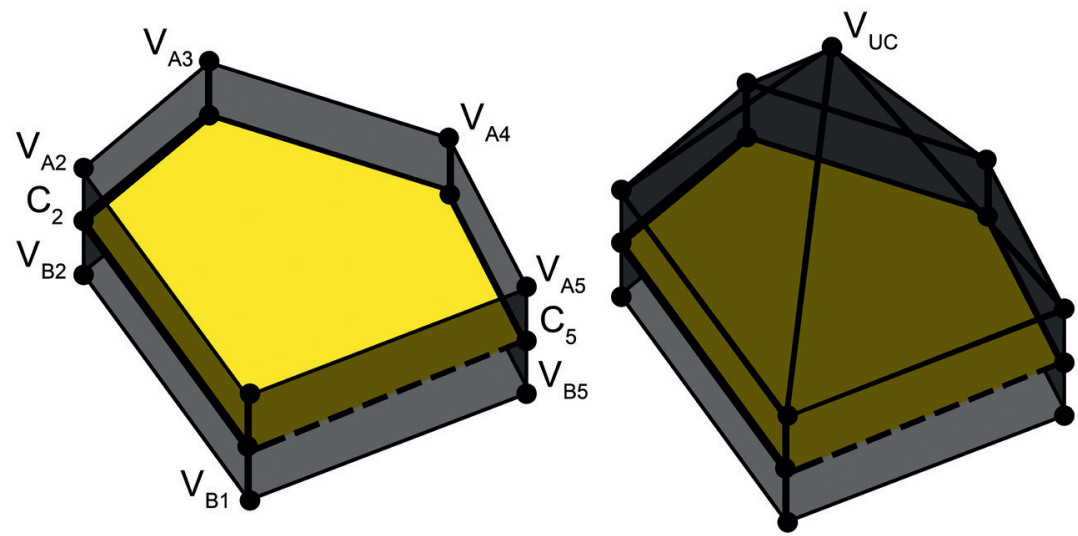

Figure 2.3 - A structure created to enclose a three-dimensional selected area. $\mathbf{V}_{\mathrm{A}(\text { (i) }}$ and $\mathbf{V}_{\mathrm{B} \text { (i) }}$ together with the upper centroid $\left(\mathbf{V}_{\mathrm{UC}}\right)$ and the lower centroid ( $\mathbf{V}_{\mathrm{LC}}$, not visible) form a structure that will cover all the faces from the surface mesh (yellow) within this structure.

Next, the final shape of the resection volume was derived from the selected area. This was achieved by creating a new cone-like shape, the base of which was formed by connecting the vertices $\mathbf{P}_{\mathrm{v}(\mathrm{i})}$ at the border of the selected area (Figure 2.4). The apex $\mathbf{P}_{c}$ was located below, in the center, and orthogonal to the mean of all the faces of the selected area (Figure 2.5). To enable more complex resection shapes, the connecting edge between the area vertices $\mathbf{P}_{\mathrm{v}(\mathrm{i})}$ and the apex $\mathbf{P}_{\mathrm{c}}$ was separated into two parts by inserting halfway vertices $\mathbf{P}_{\text {hw (i) }}$. The resulting mesh could be adjusted interactively to the desired incision shape by (a) changing the depth of the apex in a direction orthogonal to the selected area; (b) scaling the halfway vertices in a direction parallel to the selected area, and (c) changing the depth of this plane. By applying this method, both elongated and round resection volumes could be created. 


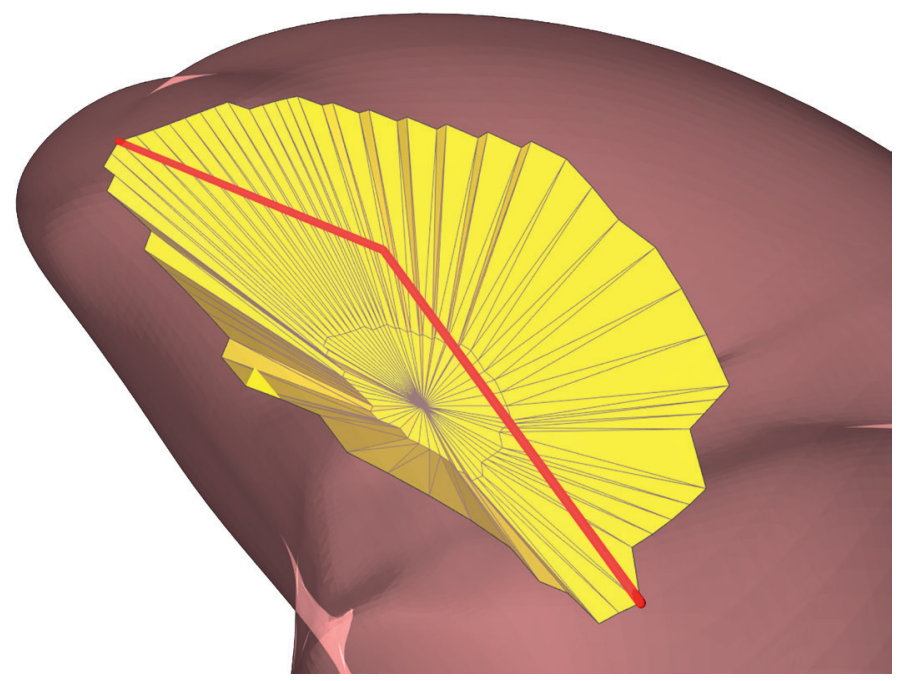

Figure 2.4 - A visual representation of the selected resection volume. The yellow volume is the volume that is about to be removed. The red line indicates over which line the future resection will be closed.

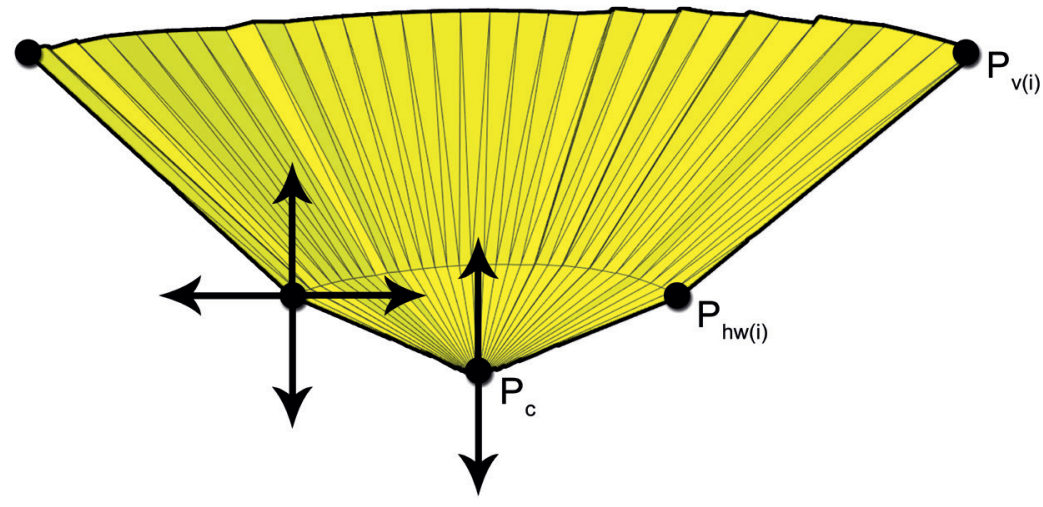

Figure 2.5 - A schematic cross-section of the resection volume. $\left(\mathbf{P}_{\mathrm{hw}(\mathrm{i})}\right)$ can be moved in the direction of, and perpendicular to the normal of all the selected faces. $\mathbf{P}_{\mathrm{c}}$ can only be moved in the direction of the normal. 


\section{Virtual suturing}

After subtracting the created resection volume from the surface mesh, the cubic FE model was generated according to this new surface mesh shape, as described in the Materials section under the heading 'The model'. Vector fields containing muscle locations and directions were added to the FE model, but not in the resected area. These vector fields were only converted to element-muscles until after the suturing procedure.

Next, the resection was closed. This was divided into two stages. In the first stage, suturing was simulated in a similar way to clinical intraoperative conditions. This was accomplished by pulling the longest edges of the resection volume towards each other using point forces located at the vertices on the edge $\left(\mathbf{P}_{\mathrm{v}(\mathrm{i})}\right)$ and halfway between the edge and bottom $\left(\mathbf{P}_{\mathrm{hw}(\mathrm{i})}\right)$ of the resection (marked by green and red arrows respectively in Figure 2.6a-b).

To create balanced forces on the opposite sides of the longest edge, a virtual plane was created at the longest axial direction of the resection. For each point force, a target was created that was the projection of the point force on the plane (marked by green and red dots in Figure 2.6a-c). This target was then corrected for the length of the resection's wall and the location of the nearest target points from the opposite side of the resection, to ensure a smooth post-operative surface. Subsequently, a simulation was run wherein the point forces move towards their targets while applying a manually adjustable force. In addition, these forces were lowered exponentially with each iteration as they reached their target to prevent the point forces from overshooting their target. During the approach of both sides of the embedded surface mesh, the FE mesh does not self-collide, as only the surface mesh can collide (Figure 2.6b). 

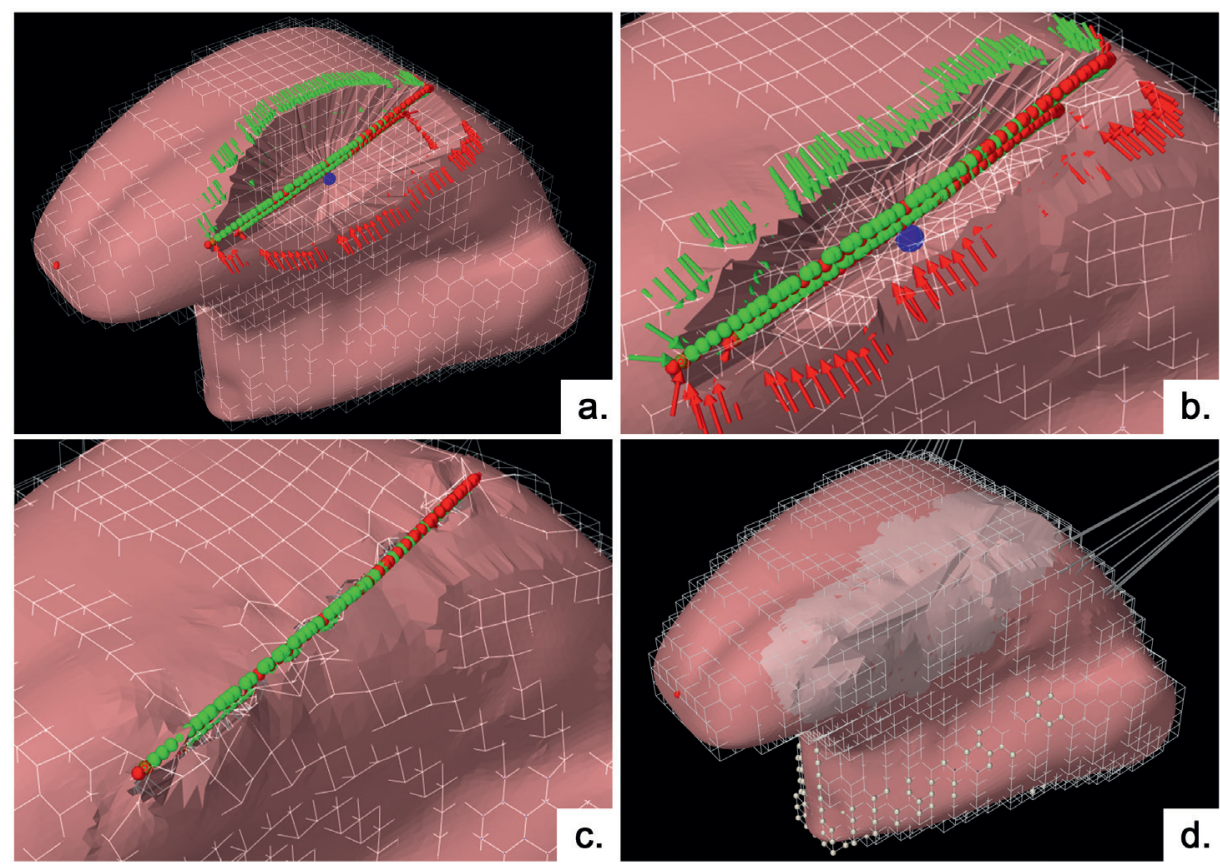

Figure 2.6 - A visual representation of the suturing procedure using the surgical tool. a-b: The red and green point forces (arrows) will move to their targets points (bullets) on an invisible plane located on the longest axial direction of the resection. c: The pairs of points on the edges that were closest to each other were bounded by a virtual knot. d: Post-operative mesh using a new generated FE mesh. The lighter area is marked as fibrosis in this particular case.

To ensure that the edges on both sides of the resection were fully aligned, the pairs of points on the edges that were closest to each other were bounded by a virtual knot. By running a simulation for a couple of seconds without any forces other than gravity, the tongue tissue moved to an equilibrium state (Figure 2.6c). Using MeshLab [25], the remaining hole in the mesh was closed. At this point, the FE mesh was discarded.

\section{Post-operative model}

The suturing procedure altered the shape of the surface mesh and muscle vector field. Based on this surface mesh, a new FE model was generated. Muscleelements were created from the altered vector fields, and using the information about the location of the resection, fibrosis (scar tissue) was added to the model (Figure 2.6d). Not much is known about the extent of fibrosis and the associated changes of the tissue properties. We will evaluate the effects of a small and large fibrotic area of 7 and $14 \mathrm{~mm}$, respectively, from the location of the resection. In Buchaillard et al. [16] a stiffened free flap reconstruction was simulated by using 6 times the original stiffness. We compared the effects of 6,12 and 24 times the initial stiffness on tongue motions. 


\subsection{EVALUATION}

\section{Comparison with the original model}

In order to compare our model to the model of Buchaillard et al. [15], we estimated the activity of each muscle from their model by means of an inverse simulation technique. Every single (bilateral) muscle of the Buchaillard et al. [15] model was activated to $50 \%$ of its maximum force or just before the model became unstable. Via the inverse simulation, the tongue tip of our model aimed to reach the same point as the tongue tip of the Buchaillard et al. [15] model, using the same muscle. As the vertical and transverse muscles mainly control the width of the tongue (in the coronal plane), the inverse simulation for those muscles will be done with 2 points on both sides of the tongue instead of the tongue tip.

\section{Evaluation of various element sizes}

The number of the cubic elements that can be used for the FE model is limited by computational power and time. The smaller the elements, the better the representation of the surface. However, the more elements, then the longer computation time. We will compare models with element sizes $16 \mathrm{~mm}^{3}, 39 \mathrm{~mm}^{3}$, and $141 \mathrm{~mm}^{3}$ by activating every muscle bilaterally. Element sizes larger than 141 $\mathrm{mm}^{3}$ are too large to follow the shape of the tongue and element sizes smaller than $16 \mathrm{~mm}^{3}$ will take longer than 50 seconds for 1 second of simulation on an IntelXeon V5 @3.50 GHz.Therefore, anelement size of $16 \mathrm{~mm}^{3}$ is, the smallest sizewewilluse.

\section{Patient case}

To demonstrate the capability of our model to mimic the effects of surgery on tongue mobility, videos of a patient with a T1 tongue carcinoma scheduled for glossectomy were captured both pre- and post-operatively, performing the following characteristic protruding tongue movements:

- forward.

- Up and downward.

- Lateralisation (left and rightward).

To determine the muscle activation signals, the measured pre-operative extreme positions were used for the inverse simulation. The tongue tip of the preoperative model was targeted to set a path towards these positions, resulting in the corresponding muscle activations. This muscle activation pattern was used in the post-operative forward simulation using various levels of stiffness and fibrosis volumes.

The maximum distance of the tongue tip of the model was measured in the predetermined directions. Because no post-operative MRI data of the patient was available, no comparison between pre-and post-operative shapes was possible. 
Therefore, the resulting shapes are only assessed qualitatively by comparing them to stills of the pre- and post-operative video of the patient. The frames were scaled by calibrating the width of the nostrils of the pre-and post-operative image.

\section{$2.4 \quad$ RESULTS}

\section{Comparison with the original model}

Figure 2.7 shows the deformation of the Buchaillard et al. [15] model after activation of a single muscle and the results of inverse simulations using our model targeting at the tip or the sides of the Buchaillard et al. [15] model. Activation of the mylohyoid or geniohyoid muscle did not change the shape of the tongue in a noticeable way and was therefore left out of this comparison. Most of the deformations of the two models were almost indistinguishable from one another, except for the hyoglossus and the superior longitudinal muscle that could not move the tongue apex of our model to the same position of Buchaillard's model [15].

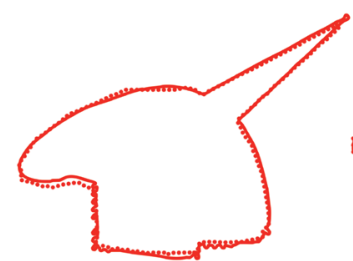

Anterior

Genioglossus

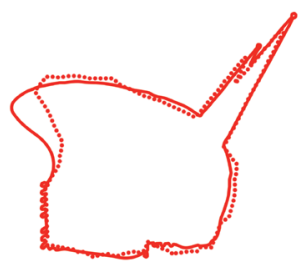

Hyoglossus

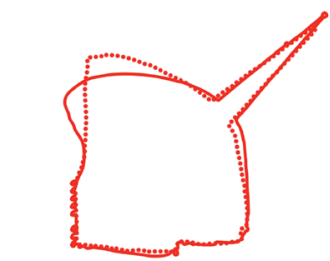

Superior Longitudinal

Muscle

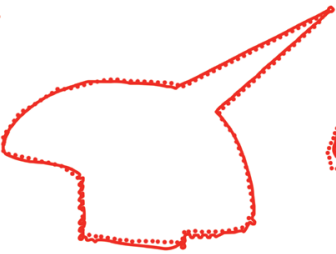

Middle

Genioglossus

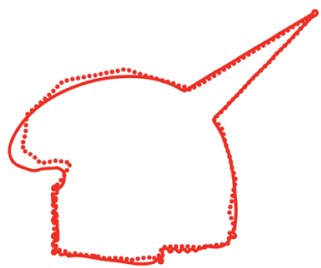

Styloglossus

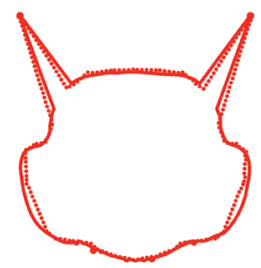

Vertical Muscle

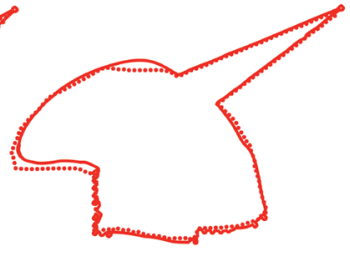

Posterior Genioglossus

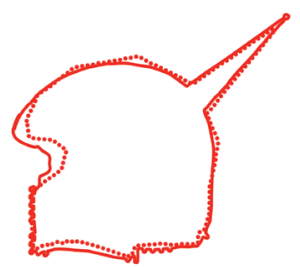

Inferior Longitudinal Muscle

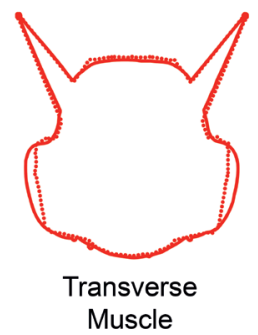

Figure 2.7 - Sagittal or coronal delineated view of the Buchaillard (2009) model (dotted lines) for each activated muscle and our model (solid line) reaching the same point with the tip (sagittal view) or the sides (coronal view) using inverse modeling. 


\section{Evaluation of various element sizes}

Figure 2.8 shows the movement of the apex in two directions after activation of the muscle. The maximum difference between $16 \mathrm{~mm}^{3}$ and $39 \mathrm{~mm}^{3}$ elements was maximally $1 \mathrm{~mm}$, however, the model with larger elements showed movements up to $4 \mathrm{~mm}$. This movement was mainly in the direction of gravity (downwards).
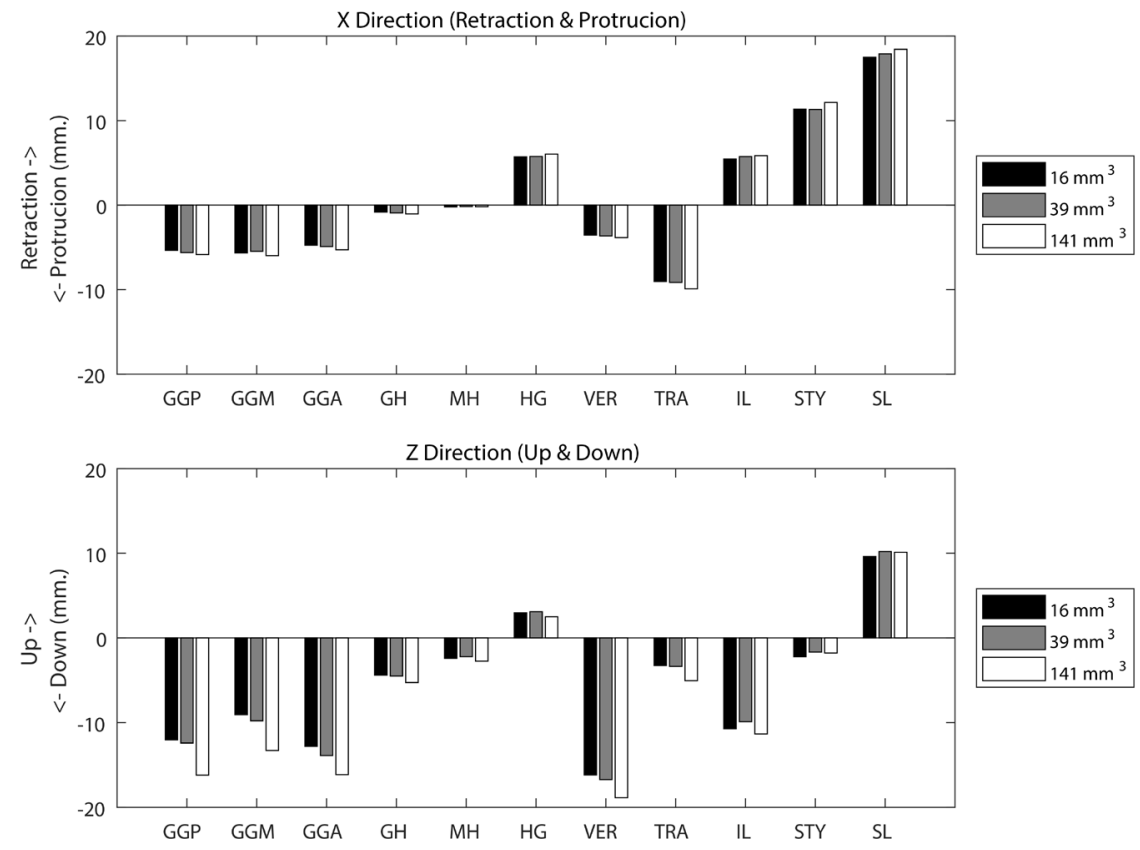

Figure 2.8 - Upper graph: The positive and negative $X$-axis represents a displacement of the tip of the tongue in a forward protruding and retracting movement of the tongue, respectively, using elements of 16, 39 and $141 \mathrm{~mm} 3$. Lower graph: The positive and negative Z-axis represents a displacement in respectively the up and down direction.

\section{Patient Case}

Figure 2.9 shows the drawing of the plan made by a surgeon, the selection made using the surgery tool, and the resulting post-operative model. The grey area represents the extension of fibrosis. The numerical results of the forward simulations are given in Figure 2.10. The most prevalent effect was the decline in motion in all directions when the stiffness and diameter of the fibrotic area of the post-operative tongue were increased. However, the non-fibrotic version of the model showed a different behavior as the ability to retract increased after surgery. Another interesting phenomenon can be seen in the left and rightward protrusion. Without fibrosis, the post-operative tongue could not move as far to the right as it did to the left, as muscles in this patient were removed from the right side of the tongue. However, when fibrosis was added, the opposite occurred. Further stiffening of the resection volume only affected the motion to the left, but 
when the size of the fibrotic area was increased both sides became affected. The right and upward protrusion were hardly affected by fibrosis of $7 \mathrm{~mm}$ while that of $14 \mathrm{~mm}$ did have a significant effect. The largest decrease in motion, compared to the healthy tongue, was visible in the left and downward protrusion.

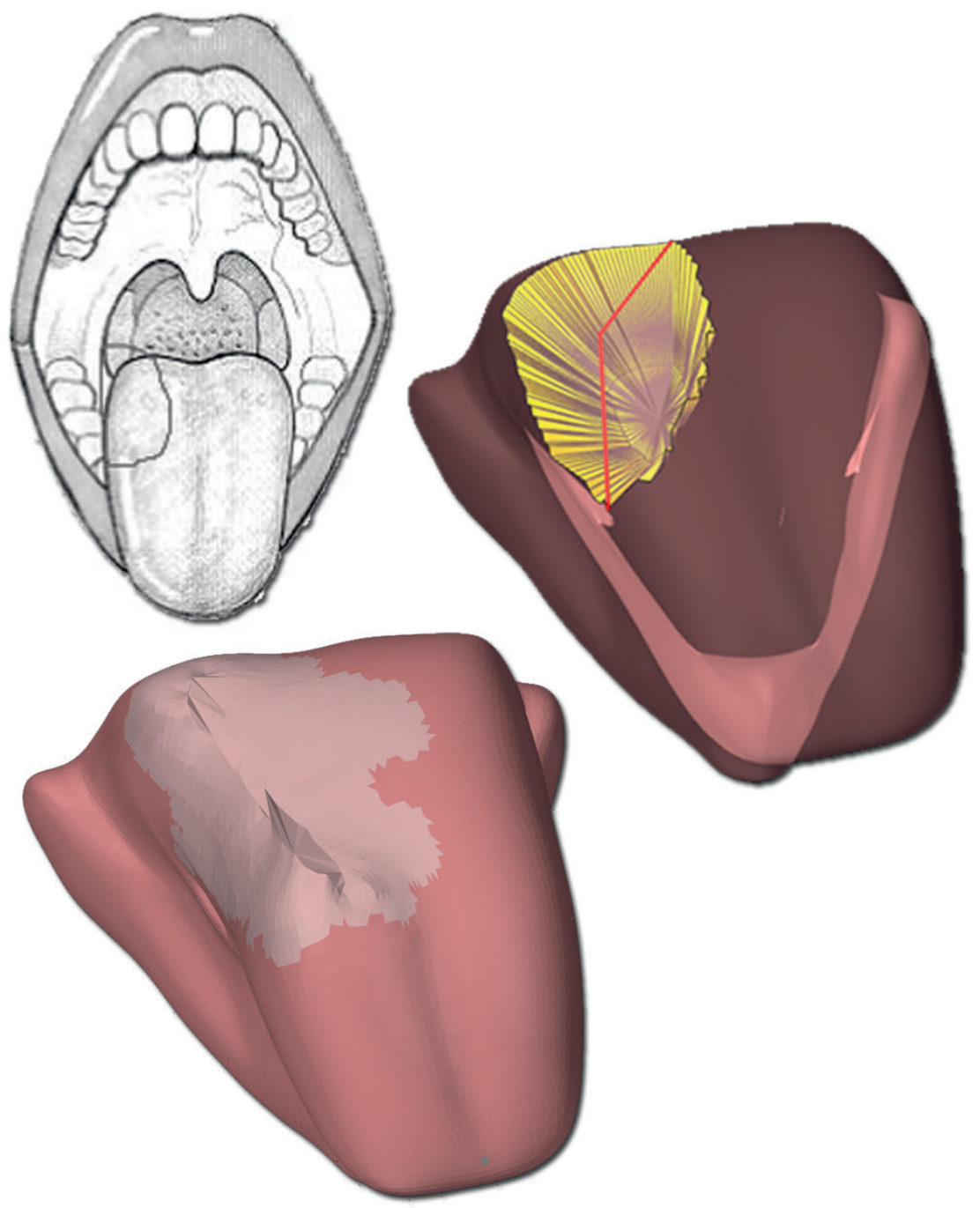

Figure 2.9 - From top to bottom: preoperative drawing of the surgical area, selected resection volume on the preoperative model and the post-operative model. The grey area on the post-operative model is the visual representation of scar tissue. 

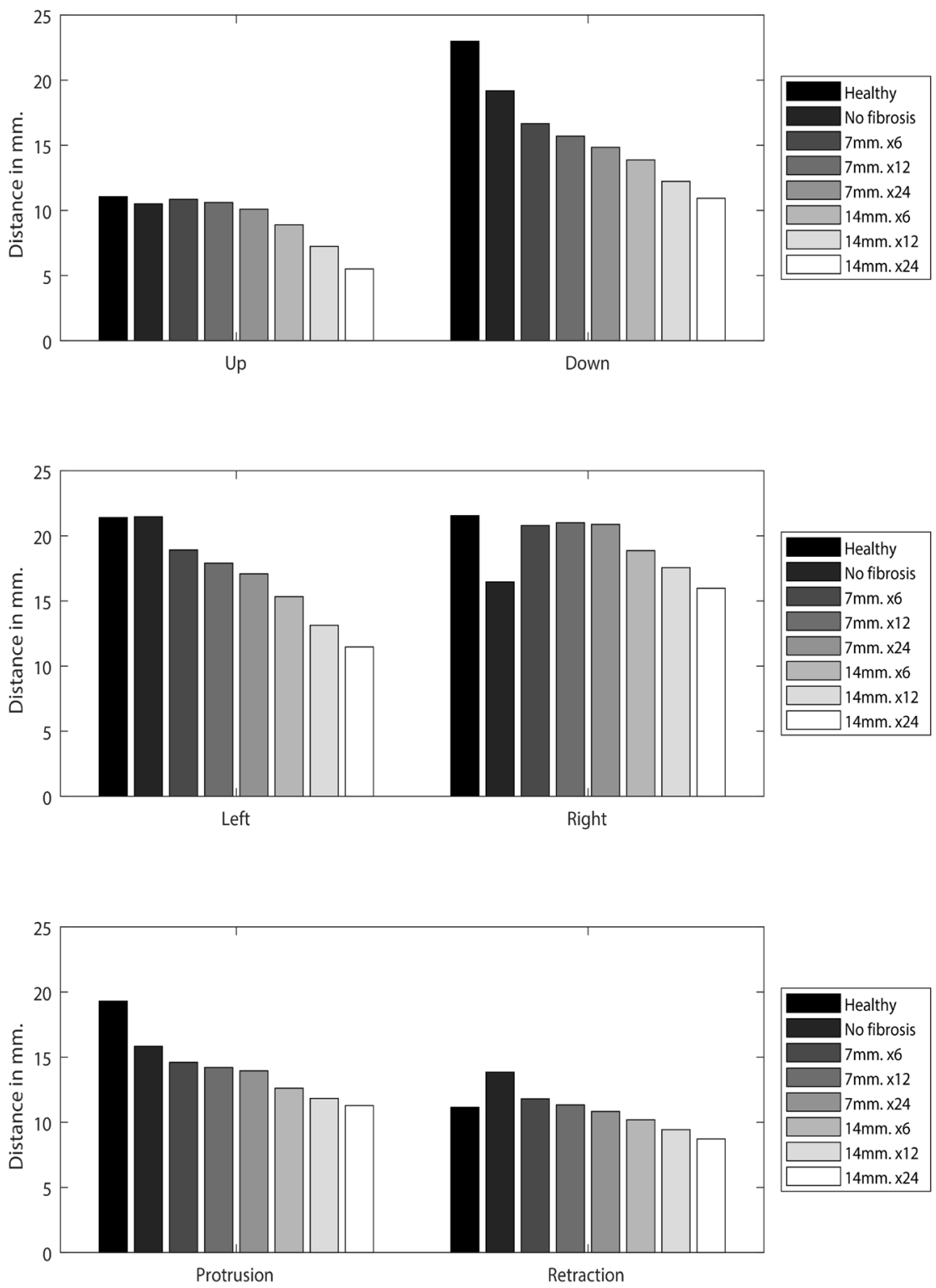

Figure 2.10 - The traveled distance in millimeter of different versions of the model while performing six different movements. The first part of the graph labels indicates the extension of fibrosis ( 7 or 14 $\mathrm{mm}$.) and the second part indicated a multiplication factor for the amount of stiffness compared to the "healthy tongue" (6,12 and 24 times the normal stiffness) 
In Figure 2.11, the pre- and post-operative differences of the patient are shown while protruding the tongue to the left or down. The post-operative tongue tip positions, projected on the pre-operative image, are indicated by the two small perpendicular lines. Both maneuvers are impaired and showed a significant decrease in motion about $30 \%$. No noticeable differences were found in up, right or forward protrusion and therefore not shown in the Figure. Both the biomechanical model and the patient revealed that the effect of surgery was most distinct in the left (contralateral) and downward protrusion.
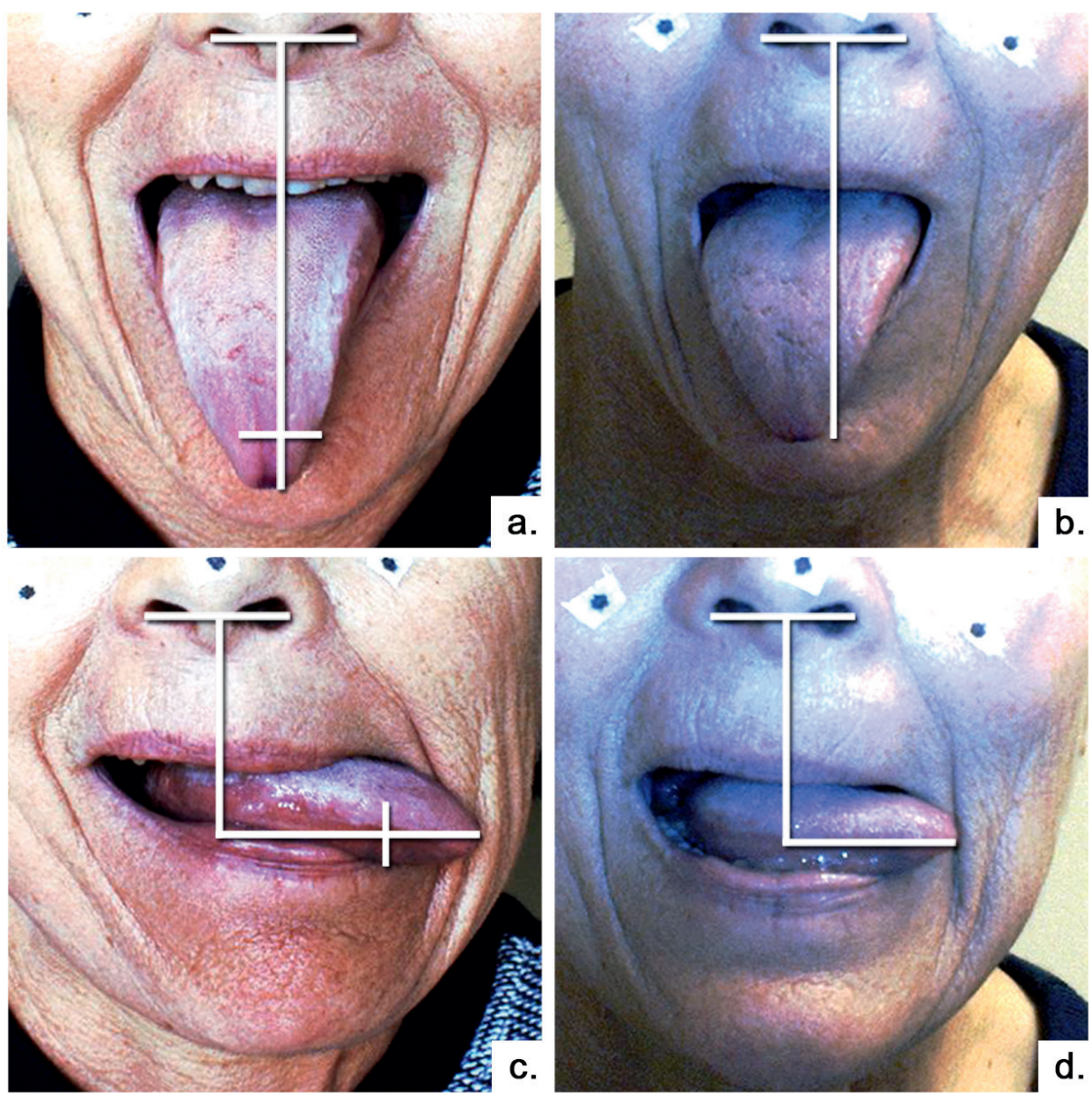

Figure 2.11 - Left: preoperative video stills from down and leftward protrusion of the tongue. The horizontal white line represents the magnitude of motion of the tongue in a certain direction seen from the nostrils. An extra vertical line on top of the horizontal line projects the post-operative magnitude of motion on the preoperative video stills. Right: Post-operative video stills. The video stills are printed with written permission of the patient. 


\subsection{DISCUSSION AND CONCLUSION}

In this study, a method to perform virtual surgery on a biomechanical tongue is presented. A user-friendly tool was created to delineate and shape a resection volume. The virtual resection, suturing of the resection, and formation of scar tissue was accomplished through biomechanical modeling. The evaluation showed that the movements of the model were comparable with the model of Buchaillard et al. [15] and that those movements were not significantly altered within a reasonable range when a different element size was chosen. Application of the tool to a single patient case, in which post-surgical movement of the patient's tongue could be simulated, gave a proof of concept, demonstrating the potential of the tool.

\section{The model}

The surface mesh of the model is a smoothed version of the Buchaillard et al. [15] model, which neither uses an unedited segmented tongue nor uses personalized muscle locations of the tongue [22]. As demonstrated in this paper, this was sufficient to predict the overall effect of surgery at a certain location. However, for quantifying motion impairment and effective validation, a truly personalized model is required. The tongue mesh and muscle bundles must be shaped to the patient's tongue by using, for instance, MRI data. Techniques such as Diffusion Tensor Imaging (DTI) can be used to image and subsequently personalize the neural and muscular structures $[29,30]$.

In literature, there is still a debate on how to simulate the functional muscular subdivision. Some FE models described in literature divide the genioglossus in the anterior, middle and posterior part [17,31-33], while other literature shows that there are also arguments to separate the genioglossus into a horizontal and oblique subdivision [34-36]. There are also arguments to subdivide the intrinsic muscles into multiple functional regions. For validating our new way of generating an FE model, to enable virtual surgery, it was necessary to use the same muscle divisions as in Buchaillard et al. [15]. In addition, van Alphen et al. [37] showed that the tongue muscle innervation patterns can differ greatly among subjects, which makes it difficult to ascertain that specific muscles are denervated by surgical procedures. However, from our longstanding clinical experience and other clinical research, we can conclude that gross tongue movements after a partial glossectomy are usually affected by fibrosis rather than by denervation [38]. Moreover, denervation at a local site seems effectively compensated at a central cerebral level [39]. 
This report describes a first use of a separate surface mesh and FE model to simulate surgical excision and closure of soft tissue. The separation made it possible to easily edit detailed parts of the tongue and actually remove a part from the model. This, in turn, enabled us to create virtual sutures to close the resection, which would be impossible using the low-resolution handmade models $[16,19,20]$. Figure 2.9 shows that the post-operative tongue contour accurately mimicked the in-vivo post-operative situation after primary suturing of the tongue.

However, the described modeling technique has its flaws. The FE mesh is solely made of cubic shaped hexahedral elements (Figure 2.1) because tetrahedral elements are more prone to "volumetric locking". An FE model with cubically shaped elements will not deform in the same way as an FE model with a smoother surface. For example, large deformations close to the jaw and hyoid bone attachments can result in a corrugated surface mesh. Also, sharp edges at the corners of a resection are not always well defined. It is arduous to automatically generate a hexahedral model that follows the surface with non-cubic elements, and therefore some have suggested a mixed-mesh approach. In Rohan et al. [18] this mixed mesh approach gave promising results as the use of tetrahedral elements located only on the surface of the FE did not alter the deformation of a tongue model in a serious way. Although not yet suited for our editable FE mesh approach, industrial solutions like "Bolt" [40] could be used to create more detailed hexahedral models as was done in Hermant et al. [17].

The hyperelastic material properties used in our simulations are a rough approximation from reality. Recent research is focused on creating constitutive laws to describe the viscoelastic behavior of (tongue) tissue [41]. These new insights, provided they don't significantly increase computational time, could improve the simulated tissue response in the model in the future.

\section{Evaluation}

The comparison of our model to the model of Buchaillard et al. [15] showed that, despite the coarse and substantially different FE Mesh, both models deformed in an almost identical way (Figure 2.7). However, the upward movement when activating the hyoglossus and superior longitudinal muscle was reduced in comparison to the Buchaillard et al. [15] model. One explanation was that, because of the cubic shaped elements, some FE nodes at the surface were only connected to one element. The movement induced in those elements did not entirely contribute to the movement of the tongue. Another explanation was that not all the jaw attachments were located at the same positions as a result of the coarse FE mesh. 
The comparison between the model with $16 \mathrm{~mm}^{3}$ and $39 \mathrm{~mm}^{3}$ elements showed hardly any difference in movement between the two (Figure 2.8). With the elements larger than $141 \mathrm{~mm}^{3}$ it becomes harder to create a shape resembling the surface mesh, but even with this size the simulation still showed comparable movements upon muscle activation. Therefore, we can conclude that the size of the elements, within reasonable limits, does not affect the simulations at this stage of development, but that a small element size is always preferred since they result in more detailed shapes.

The patient case demonstrated that the surgery tool is a good starting point in creating a usable post-operative model to predict surgical impairment. It also showed the importance of choosing the right location and the right amount of stiffness. The extension of fibrosis to $14 \mathrm{~mm}$ caused the stiffened area to expand across the midline of the tongue and resulted in more impaired movement when protruding in the up and rightward direction (Figure 2.10). In the non-realistic case without fibrosis, the left-right difference is caused solely by the deformed model and removed muscle parts. By increasing the stiffness of fibrosis the leftright difference is reversed. This contralateral motion impairment was clearly simulated in our model and was comparable to our patient and patients of a study performed by van Dijk et al. [42]. This underlines the importance of better in-vivo research on tissue properties, fibrosis, and muscle morphology.

The qualitative comparison showed promising results regarding this technique and therefore a quantitative validation is desired. Therefore, we started developing a new protocol, comparable to the one of van Dijk et al. [42], to track the patient's tongue motion pre- and post-operatively. When the model has proven to be a good predictor of post-treatment functionality also other applications than patient counseling could be considered. In the field of speech and swallowing rehabilitation, for example, the personalized post-operative model can be used to point out alternative muscle sets to compensate for the loss of other muscles via inverse modeling. The speech therapist can use the model to determine which muscles to train in order to maximize the effect of training. Also, once we have achieved an acceptable implementation of fibrosis in the model, various ways of wound closure and their influences on the post-operative motion can be analyzed in the process of clinical decision making. We believe that these simulations will eventually provide a strong support for the patient and the treating physicians in both shared decision-making and rehabilitation of the patient. 


\subsection{REFERENCES}

1. Warnakulasuriya S. Global epidemiology of oral and oropharyngeal cancer. Oral Oncol. Elsevier Ltd; 2009;45: 309-316. doi:10.1016/j.oraloncology.2008.06.002

2. Gupta N, Gupta R, Acharya AK, Patthi B, Goud V, Reddy S, et al. Changing Trends in oral cancer - a global scenario. Nepal J Epidemiol. International Nepal Epidemiological Association; 2016;6: 613-619. doi:10.3126/nje.v6i4.17255

3. Moore SR, Johnson NW, Pierce a M, Wilson DF. The epidemiology of mouth cancer: a review of global incidence. Oral Dis. 2000;6: 65-74. doi:10.1111/j.1601-0825.2000. tb00104.x

4. Costa Bandeira AK, Azevedo EHM, Vartanian JG, Nishimoto IN, Kowalski LP, CarraraDe Angelis E. Quality of life related to swallowing after tongue cancer treatment. Dysphagia. 2008;23: 183-192. doi:10.1007/s00455-007-9124-1

5. Van Der Molen L, Van Rossum MA, Burkhead LM, Smeele LE, Hilgers FJM. Functional outcomes and rehabilitation strategies in patients treated with chemoradiotherapy for advanced head and neck cancer: A systematic review. Eur Arch Oto-Rhino-Laryngology. 2009;266: 889-900. doi:10.1007/s00405-008-0817-3

6. Sessions DG, Spector GJ, Lenox J, Haughey B, Chao C, Marks J. Analysis of treatment results for oral tongue cancer. Laryngoscope. 2002;112: 616-25. doi:10.1097/00005537-200204000-00005

7. Shah JP, Gil Z. Current concepts in management of oral cancer - Surgery. Oral Oncol. 2009;45: 394-401. doi:10.1016/j.oraloncology.2008.05.017

8. Yudkin JS, Kavanagh J, McCormack JP. Guidelines for treating risk factors should include tools for shared decision making. BMJ. 2016;353. doi:10.1136/bmj.i3147

9. Braillon A. Independence is critical for shared decision making. BMJ. 2017;358. doi:10.1136/bmj.j3286

10. Kreeft AM, Tan IB, van den Brekel MWM, Hilgers FJ, Balm AJM. The surgical dilemma of 'functional inoperability' in oral and oropharyngeal cancer: current consensus on operability with regard to functional results. Clin Otolaryngol. 2009;34: 140-146. doi:10.1111/j.1749-4486.2009.01884.x

11. Gerard JMM, Ohayon J, Luboz V, Perrier P, Payan Y. Non-linear elastic properties of the lingual and facial tissues assessed by indentation technique: Application to the biomechanics of speech production. Med Eng Phys. Elsevier; 2005;27: 884-892. doi:10.1016/j.medengphy.2005.08.001

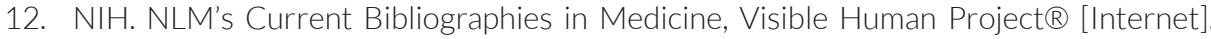
2007 [cited 3 Jul 2018]. Available: http://www.nlm.nih.gov/archive/20120907/pubs/ cbm/visible_human_2007.html

13. Wilhelms-TricaricoR. Developmentofatongueandmouthfloormodelfornormalizationand biomechanical modelling. Proceedings of the Fifth Speech Production Seminar and CREST Workshop on Models of Speech Production. Kloster Seeon, Bavaria; 2000. pp. 141-148.

14. Vogt F, Lloyd JE, Buchaillard S, Perrier P, Chabanas M, Payan Y, et al. Efficient 3D finite element modeling of a muscle-activated tongue. Lect Notes Comput Sci. 2006; 19-28. doi:10.1007/11790273_3

15. Buchaillard S, Perrier P, Payan Y. A biomechanical model of cardinal vowel production: muscle activations and the impact of gravity on tongue positioning. J Acoust Soc Am. 2009;126: 2033-2051. doi:10.1121/1.3204306 
16. Buchaillard S, Brix M, Perrier P, Payan Y. Simulations of the consequences of tongue surgery on tongue mobility: Implications for speech production in post-surgery conditions. Int J Med Robot Comput Assist Surg. 2007;3: 252-261. doi:10.1002/ rcs.142

17. Hermant N, Perrier P, Payan Y. Human Tongue Biomechanical Modeling. In: Payan Y, Ohayon JBT-B of LO, editors. Biomechanics of Living Organs: Hyperelastic Constitutive Laws for Finite Element Modeling. Oxford: Academic Press; 2017. pp. 395-411. doi:10.1016/B978-0-12-804009-6.00019-5

18. Rohan P-Y, Lobos C, Nazari M a., Perrier P, Payan Y. Finite element models of the human tongue: a mixed-element mesh approach. Comput Methods Biomech Biomed Eng Imaging Vis. 2016;00: 1-11. doi:10.1080/21681163.2015.1105760

19. Fujita S, Dang J, Suzuki N, Honda K. A Computational Tongue Model and its Clinical Application. Oral Sci Int. 2007;4: 97-109. doi:10.1016/S1348-8643(07)80004-8

20. Van Alphen MJA, Kreeft AM, Van Der Heijden F, Smeele LE, Balm AJM. Towards virtual surgery in oral cancer to predict postoperative oral functions preoperatively. Br J Oral Maxillofac Surg. British Association of Oral and Maxillofacial Surgeons; 2013;51: 747751. doi:10.1016/j.bjoms.2013.06.012

21. Kreeft AM, Van Der Molen L, Hilgers FJ, Balm AJ. Speech and swallowing after surgical treatment of advanced oral and oropharyngeal carcinoma: A systematic review of the literature. Eur Arch Oto-Rhino-Laryngology. Springer; 2009;266: 1687-1698. doi:10.1007/s00405-009-1089-2

22. Gerard J-M, Wilhelms-Tricarico R, Perrier P, Payan Y, Gérard J-M, Wilhelms-Tricarico R, et al. A 3D dynamical biomechanical tongue model to study speech motor control. Res Dev Biomech. 2003;1: 49-64.

23. Stavness I, Lloyd JE, Fels S. Automatic prediction of tongue muscle activations using a finite element model. J Biomech. Elsevier; 2012;45: 2841-2848. doi:10.1016/j. jbiomech.2012.08.031

24. Lloyd JE, Stavness I, Fels S. ArtiSynth: A Fast Interactive Biomechanical Modeling Toolkit Combining Multibody and Finite Element Simulation. Studies in Mechanobiology, Tissue Engineering and Biomaterials. 2012. pp. 355-394. doi:10.1007/8415_2012_126

25. Cignoni P, Cignoni P, Callieri M, Callieri M, Corsini M, Corsini M, et al. MeshLab: an Open-Source Mesh Processing Tool. Sixth Eurographics Ital Chapter Conf. 2008; 129136. doi:10.2312/LocalChapterEvents/ItalChap/ItalianChapConf2008/129-136

26. Boyé S, Guennebaud G, Schlick C. Least squares subdivision surfaces. Comput Graph Forum. 2010;29: 2021-2028. doi:10.1111/j.1467-8659.2010.01788.x

27. Lloyd JE. ArtiSynth Reference Manual [Internet]. 2014 [cited 5 Jun 2018]. Available: https://www.artisynth.org/Documentation/ArtisynthRefManual

28. Blemker SS, Pinsky PM, Delp SL. A 3D model of muscle reveals the causes of nonuniform strains in the biceps brachii. J Biomech. 2005;38: 657-665. doi:10.1016/j. jbiomech.2004.04.009

29. Gilbert RJ, Wedeen VJ, Magnusson LH, Benner T, Wang R, Dai G, et al. Threedimensional myoarchitecture of the bovine tongue demonstrated by diffusion spectrum magnetic resonance imaging with tractography. Anat Rec - Part A Discov Mol Cell Evol Biol. 2006;288: 1173-1182. doi:10.1002/ar.a.20387

30. Gaige TA, Benner T, Wang R, Wedeen VJ, Gilbert RJ. Three dimensional myoarchitecture of the human tongue determined in vivo by diffusion tensor imaging with tractography. J Magn Reson Imaging. 2007;26: 654-661. doi:10.1002/jmri.21022 
31. Harandi NM, Woo J, Stone M, Abugharbieh R, Fels S. Subject-specific biomechanical modelling of the tongue: Analysis of muscle activations during speech. Proceedings of the 10th International Seminar on Speech Production, ISSP 2014. 2014. pp. 174-177.

32. Wu X, Dang J, Stavness I. Iterative method to estimate muscle activation with a physiological articulatory model. Acoust Sci Technol. 2014;35: 201-212. doi:10.1250/ ast.35.201

33. Dabbaghchian S, Arnela M, Engwall O, Guasch O, Stavness I, Badin P, et al. Using a Biomechanical Model and Articulatory Data for the Numerical Production of Vowels. INTERSPEECH. 2016;m: 3569-3573. doi:10.21437/Interspeech.2016-1500

34. Mu L, Sanders I. Human tongue neuroanatomy: Nerve supply and motor endplates. Clin Anat. 2010;23: 777-791. doi:10.1002/ca.21011

35. Sanders I, Mu L. A three-dimensional atlas of human tongue muscles. Anat Rec. 2013;296: 1102-1114. doi:10.1002/ar.22711

36. Honda K, Murano EZ, Takano S, Masaki S, Dang J. Anatomical considerations on the extrinsic tongue muscles for articulatory modeling. Proceedings of Meetings on Acoustics. 2013. pp. 60-270. doi:10.1121/1.4800262

37. Van Alphen MJAJA, Eskes M, Smeele LEE, Balm AJMJM, van der Heijden F. In vivo intraoperative hypoglossal nerve stimulation for quantitative tongue motion analysis. Comput Methods Biomech Biomed Eng Imaging Vis. Taylor \& Francis; 2017;5: 409415. doi:10.1080/21681163.2015.1072056

38. Speksnijder CM, van der Bilt A, van der Glas HW, Koole R, Merkx MAW. Tongue function in patients treated for malignancies in tongue and/or floor of mouth; a one year prospective study. Int J Oral Maxillofac Surg. International Association of Oral and Maxillofacial Surgery; 2011;40: 1388-1394. doi:10.1016/j.ijom.2011.09.003

39. Mosier K, Liu WC, Behin B, Lee C, Baredes S. Cortical adaptation following partial glossectomy with primary closure: Implications for reconstruction of the oral tongue. Ann Otol Rhinol Laryngol. 2005;114: 681-687. doi:10.1177/000348940511400905

40. csimsoft. Bolt [Internet]. [cited 3 Jul 2018]. Available: http://www.csimsoft.com/ boltoverview

41. Yousefi A-AK, Nazari MA, Perrier P, Panahi MS, Payan Y. A visco-hyperelastic constitutive model and its application in bovine tongue tissue. J Biomech. 2018;71: 190-198. doi:10.1016/j.jbiomech.2018.02.008

42. van Dijk S, van Alphen MJA, Jacobi I, Smeele LE, van der Heijden F, Balm AJM. A New Accurate 3D Measurement Tool to Assess the Range of Motion of the Tongue in Oral Cancer Patients: A Standardized Model. Dysphagia. Springer US; 2016;31: 97-103. doi:10.1007/s00455-015-9665-7 



\title{
QUANTIFICATION OF TONGUE MOBILITY IMPAIRMENT USING OPTICAL TRACKING IN PATIENTS AFTER RECEIVING PRIMARY SURGERY OR CHEMORADIATION
}

\author{
K.D.R. Kappert \\ M.J.A. van Alphen \\ L.E. Smeele \\ A.J.M. Balm \\ F. van der Heijden
}

This chapter was published in PLOS ONE:

Kappert KDR, van Alphen MJA, Smeele LE, Balm AJM, van der Heijden F. Quantification of tongue mobility impairment using optical tracking in patients after receiving primary surgery or chemoradiation. Kimple RJ, editor. PLoS One. 2019;14: e0221593. doi:10.1371/journal.pone.0221593

This work was presented at:

- PWHHT, Amersfoort, 2018

- 15th International Symposium on Computer Methods in Biomechanics and Biomedical Engineering and the 4th Conference on Imaging and Visualization, Lisbon, 2018 


\section{ABSTRACT}

Purpose: Tongue mobility has shown to be a clinically interesting parameter on functional results after tongue cancer treatment which can be objectified by measuring the Range Of Motion (ROM). Reliable measurements of ROM would enable us to quantify the severity of functional impairments and use these for shared decision making in treatment choices, rehabilitation of speech, and swallowing disturbances after treatment.

Method: Nineteen healthy participants, eighteen post-chemotherapy patients, and seventeen post-surgery patients were asked to perform standardized tongue maneuvers in front of a 3D camera system, which were subsequently tracked and corrected for head and jaw motion. Indicators, such as the left-right tongue range and the deflection angle with the horizontal axis were extracted from the tongue trajectory to serve as a quantitative measure for the impaired tongue mobility.

Results: The range and deflection angle showed an excellent intra- and interrater reliability (ICC 0.9) The repeatability experiment showed an average standard deviation of $2.5 \mathrm{~mm}$ to $3.5 \mathrm{~mm}$ for every movement, except the upward movement. The post-surgery patient group showed a smaller tongue range and higher deflection angle overall than the healthy participants. Postchemoradiation patients showed less difference in tongue ROM compared with healthy participants. Only a few patients showed asymmetrical movement after treatment, which could not always be explained by T-stage or the side of treatment alone.

Conclusion: We introduced a reliable and reproducible method for measuring the ROM and to quantify for motion impairments, that was able to show differences in tongue ROM between healthy subjects and patients after chemoradiation or surgery. Future research should focus on measuring patients with oral cancer preand post-treatment in combination with the collection of detailed information about the individual tongue anatomy, so that the full ROM trajectory can be used to identify changes over time and to quantify functional impairment. 


\subsection{INTRODUCTION}

Head and neck cancer is the sixth most frequently occurring cancer worldwide. Carcinoma of the tongue and base of the tongue account for about $20 \%$ of all head and neck cancers [1], and incidences are rising, particularly in the oropharynx due to HPV infections [2]. Surgery is the most preferred treatment for tongue carcinomas, whereas base of tongue carcinomas are mostly treated by organ sparing radiation with or without concurrent chemotherapy [3]. In advanced cases, both treatments might seriously affect the mobility of the tongue, resulting in impaired speech, swallowing, or mastication [4,5].

The current understanding of post-treatment tongue function in clinical practice is based on anatomical and physiological reasoning and personal experience. Surgical treatment of lateral tongue carcinoma often leads to asymmetrical tongue movements [6,7]. With increasing $T$ stage this impairment becomes more outspoken and is accompanied by deterioration of speech quality and mastication function [8-10]. The organ sparing surgical chemoradiation is usually the preferred treatment for base of the tongue tumors, but in advanced cases this modality may also lead to serious functional deficits with more impact on swallowing than surgery of the mobile tongue [3,11]. Currently, it is not possible to accurately predict the functional impairments at an individual level, therefore clinical decision making, which implements expected functional sequelae, remains mainly dependent on the personal experience of the treating physician.

Tongue mobility has shown to be a clinically interesting parameter on functional results and can be objectified by measuring the Range Of Motion (ROM) [12-15]. Reliable measurements of impaired ROM would enable us to quantify the severity of functional impairments and use these for shared decision making in treatment choices, rehabilitation of speech and swallowing disturbances after treatment $[15,16]$. In addition, the ROM and other characteristics of the 3D trajectory of the tongue can be used as an input feature for biomechanical models aimed at predicting consequences of treatment [17-19]. Classical imaging techniques such as video fluoroscopy and ultrasound can visualize the tongue in a sagittal slice to evaluate the shape of the tongue in a 2D plane, but are not aimed at tracking the 3D position of the tongue [20-22]. Although MRI techniques are rapidly advancing, they are still not able to capture the 3D motion of the tongue [23-26]. Electromagnetic articulography (EMA) is a reliable technique to measure the 3D shape of the tongue over time and has, over the past decades, been used in research focussed at speech swallowing and mastication function [27-32]. It is, however still a very expensive and complicated procedure that is not comfortable for the patients. 
In 2016, our research group published a paper about a triple camera set up to assess 3D ROM information of the tongue tip as a fast, secure and accessible alternative for classical imaging techniques and EMA [15]. We showed that this was a reliable tool (intraclass correlation of over 0.9 ) for impaired tongue mobility after a partial glossectomy. By manually selecting four landmarks on the head and one on the tip of the tongue for every camera position, the distance between the interdental papilla and the tip of the tongue was determined. Using this system we were able to show impaired mobility to the contralateral side of the resection in glossectomy patients [15].

Although the triple camera set-up was sufficient for pointing out differences between patients, the technique still had some limitations: a) The maximum 3D deflection of the tongue during a specific maneuver was calculated by manual selection of the tongue tip in two $2 \mathrm{D}$ videos, thereby the full 3D trajectory information of the tongue tip was not used. b) For a left-right maneuver, the Euclidian distance was used as a measure for the ROM. When the participant showed a deviation, other than horizontal, this method tended to overestimate the horizontal deflection. c) Jaw movements were not accounted for, and d) Due to self-occlusion, the tip of the tongue was often not visible. In these cases, the ROM would be inaccurate or even incorrect.

In this study, we describe a 3D tracking tool for measuring the complete trajectory of tongue tip movements to address the aforementioned limitations of the triple camera set-up. From this trajectory, we can derive indicators which are potential quantitative measures for the mobility impairment of the tongue after treatment. This comprehensive approach would be more suitable for clinical use and input for biomechanical tongue models. The research questions of this study are:

1) What are possible indicators using a $3 D$ tracking tool for impaired mobility after tongue cancer treatment?

2) Are these indicators reliable and reproducible?

3) Can the asymmetry of the tongue mobility be quantified?

\subsection{MATERIALS AND METHODS}

\section{Participants}

To determine if the improved method can be used to objectively determine asymmetry, we included a total of 57 participants between June 2017 and December 2018. Nineteen healthy participants, Nineteen tongue carcinoma patients (tumor stages T1-T3) who had undergone a partial glossectomy followed by primary closure of the defect, and Nineteen patients with a carcinoma of the tongue base (tumor stages T1 -T4) who had been treated solely with 
chemoradiation. All healthy participants were at the age of 18 or older and did not have any history of oral cancer or other diseases that might influence the mobility of the tongue. In the two patient groups, ROM was measured at least six months after treatment. The two patient groups will later be referred to as the post-surgery and the post-chemoradiation group, respectively.

All procedures performed in studies involving human participants were in accordance with the ethical standards of the medical ethical committee of the Netherlands Cancer Institute and with the 1964 Helsinki declaration and its later amendments or comparable ethical standards. A written Informed consent was obtained from all individual participants included in the study and was approved by the medical ethical committee of the Netherlands Cancer Institute (ref:N17SWU).

\section{Experimental setup}

The experimental workflow includes acquisition, tracking, processing, and extraction of indicators for the tongue mobility impairment, which are explained in the next paragraphs and are summarized in Figure 3.1.

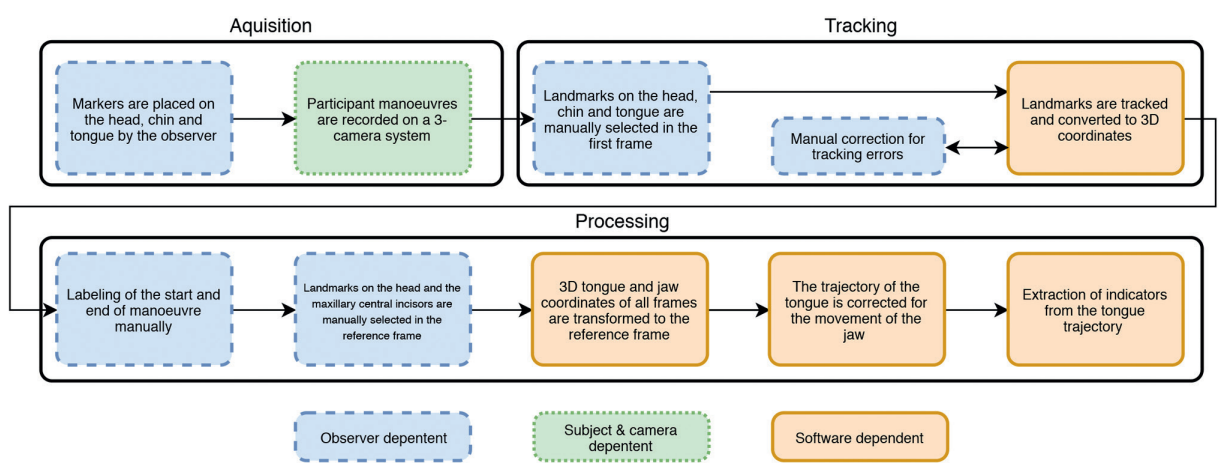

Figure 3.1 - Flowchart, summarizing the measurement steps and data processing. The color and outline refer to the actuator responsible for potential variation during a specific part of the process.

\section{Acquisition}

To measure the position of the tongue tip, a custom-made 3D camera setup was used. The setup consists of three Basler ${ }^{\circledR}$ av1000-100gc 100fps cameras, horizontally aligned, and targeted at the tongue with an angle of $20^{\circ}$ from each other (Figure 3.2a). The cameras were calibrated using 300 video frames of a checkerboard image and the Matlab ${ }^{\circledR}$ stereo-calibration tool. To enable tracking of the tongue tip, a paper marker was designed (Figure 3.2b). This marker-design features a 3D paper cube to ensure that the marker is visible from every angle. Additional markers were placed on the glabella, apex of the nose and mental region to enable tracking of the head and jaw (Figure 3.3). The caruncles of the eyes did not require external markers as they are distinguishable landmarks. 


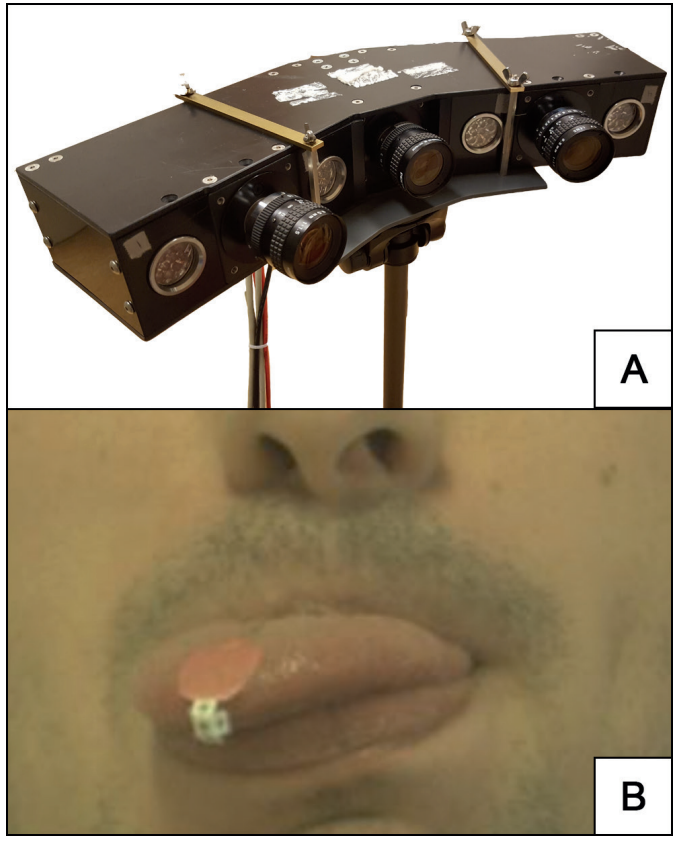

Figure 3.2 - Requisites for ROM measurement. (a) The triple camera system. (b) The tongue marker, $3 \mathrm{D}$ paper cube, placed on the tongue tip of a healthy participant.

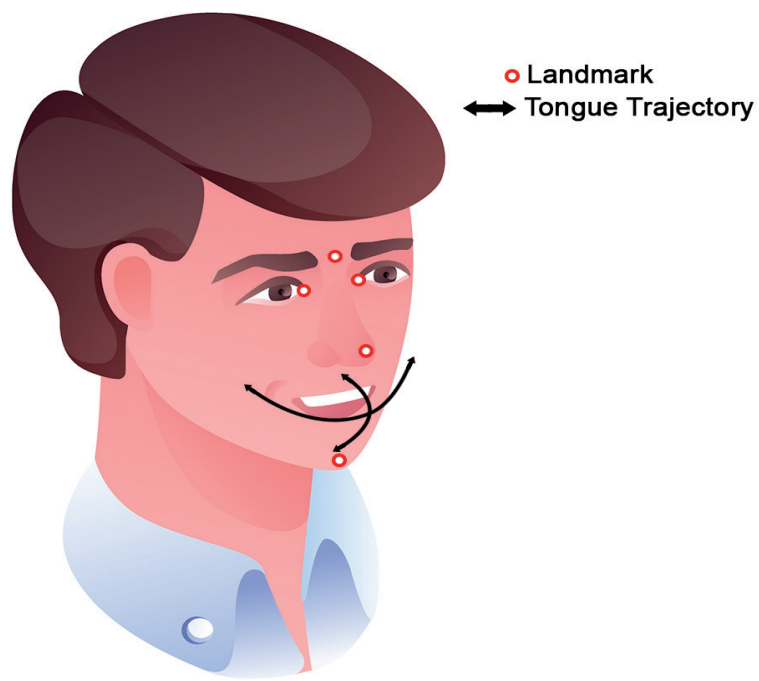

Figure 3.3 - An illustration of a participant's head and all landmarks and tongue maneuvers. The tongue maneuvers are visualized as black arrows. Markers on were placed the head, chin and nose of the participant in order to track them. The caruncles of the eyes do not require external markers as they are a distinguishable facial feature. (Illustration designed by Vectorpouch / Freepik) 
After the paper marker was placed on the tongue tip by the observer, the recording was started and participants were asked to perform three different maneuvers:

- Protrusion left to right with the tongue

- Protrusion down to up with the tongue

- Showing the maxillary central incisors

The participants were instructed to always protrude the tongue as far as possible in all directions (Figure 3.3).

\section{Tracking}

A user interface was developed to extract and process the ROM trajectory from the three videos cameras. First, the locations of six landmarks were selected: the caruncles of the eyes, glabella, nose point, tongue point and mental region (Figure 3.3). Using the Lucas Kanade tracking algorithm implemented by Matlab (MatWorks, 2018b) the six points were tracked until the end of the video. Manual interferencewaspossibletoadjustfortrackingfailures.Usingthecameracalibration parameters, calculated by the stereo-calibration tool in Matlab (MathWorks, 2018b), the 3D positions of all tracked makers over time were reconstructed. The trajectory of the tongue tip was smoothed and equidistantly resampled. Time stamps were added manually to label the start and end of the maneuvers.

\section{Processing}

The 3D trajectory of the tongue tip was processed in order to compensate for head movements. For this purpose, a reference video frame wherein the maxillary central incisors were visible was chosen. In this reference frame five points were selected: the caruncles of both eyes, in between the crowns of the maxillary central incisors, the marker on the glabella and the marker on the apex of the nose. For each video frame $i$, these points were used in a Procrustes algorithm to obtain the $4 \times 4$ transformation matrix ${ }^{\text {ref }} T(i)$ that represents the 3D pose of the head in video frame $i$ relative to the $3 \mathrm{D}$ pose of the head in the reference video frame. The transformation matrices ${ }^{\text {ree }} \mathrm{T}(i)$ were applied to the reconstructed $3 \mathrm{D}$ positions of the tongue tip and jaw so that they are all expressed in the single coordinate system, which is associated with the reference video frame. The origin of this reference coordinate system was set at the junction between the crowns of the maxillary central incisors in the reference video frame (Figure 3.4). The $\mathrm{X}$-axis was aligned with the caruncles and the $\mathrm{Y}$-axis was therefore positioned in-between the caruncles and perpendicular to the $X$-axis. The $Z$-axis was placed perpendicular to the plane formed by the caruncles and the junction between the maxillary central incisors (Figure 3.4). 


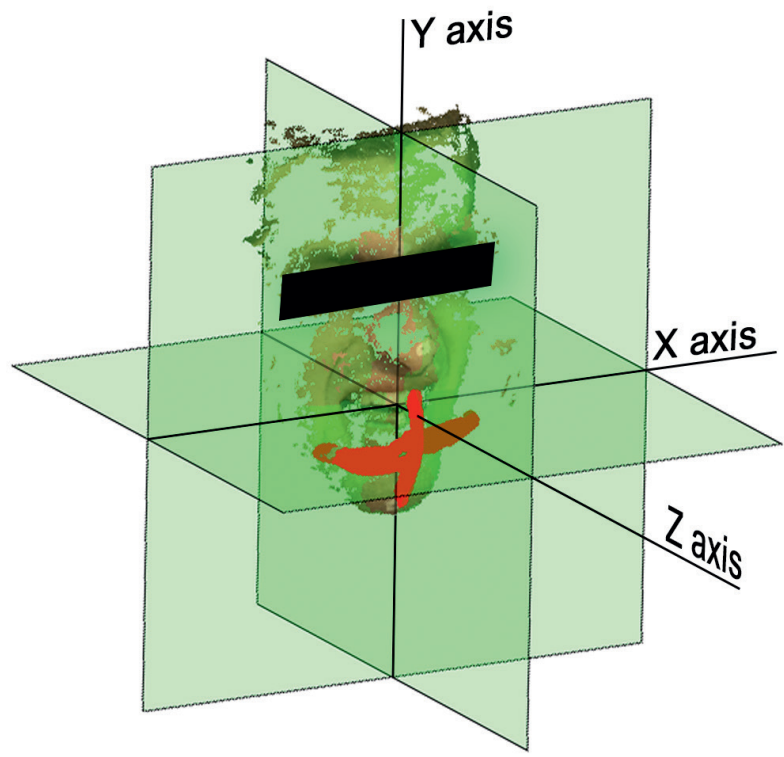

Figure 3.4 - A 3D reconstruction of a participant and its tongue trajectory. The tongue trajectory represented in red. The planes of the coordinate system are transparent green. A point cloud of the face is plotted as a reference for the location of the head.

Additionally, the trajectory of the tongue tip was corrected for jaw movement. During the down-up movement, the video frame wherein the tongue marker was closest to the horizontal plane (X-0-Z plane) was chosen as the reference video frame. The position of the jaw marker in this frame was used as a reference for jaw movement. The relative displacement of the jaw, seen from this reference point, was subtracted from the tongue tip trajectory.

\section{Indicators for tongue mobility impairment}

To interpret the resulting 3D tongue tip trajectory, aspects of this trajectory need to be translated into indicators. The following indicators were extracted from the tongue tip trajectory:

- Range $R_{l}$ and $R_{R}$ : the maximal deviation of the tongue tip in the positive and negative $\mathrm{X}$ direction, while performing the left to right protrusion respectively (Figure 3.5). This is, effectively, the extreme position of the tongue tip projected on the horizontal plane. The range of the up and down movement, $R_{u}$ and $R_{d}$, is defined likewise in the $Y$ direction. The range $R_{f}$ of the forward protrusion is defined in the $Z$ direction.

- Deflection angles to the right and the left $\left(\varphi_{r}\right.$ and $\left.\varphi_{l}\right)$ : Patients who underwent surgery often have difficulties to move the tongue tip to the desired position. A way to express this is by calculating the angle between the axis of the instructed 
direction (X-axis for left and right) and the line from the tongue tip at maximum range to the origin over the XY plane (Figure 3.5).

The 3D trajectory expressed in the coordinate system enables calculation of areas and 3D volumes that could cover all, or parts, of the maneuver. Since the current dataset only contains healthy or post-treatment measurements it is not possible to explore and demonstrate the added value in objectifying functional loss. Therefore, we focus on asymmetries between participants and overall differences between groups only using the ranges and deflection angles of the maneuvers.

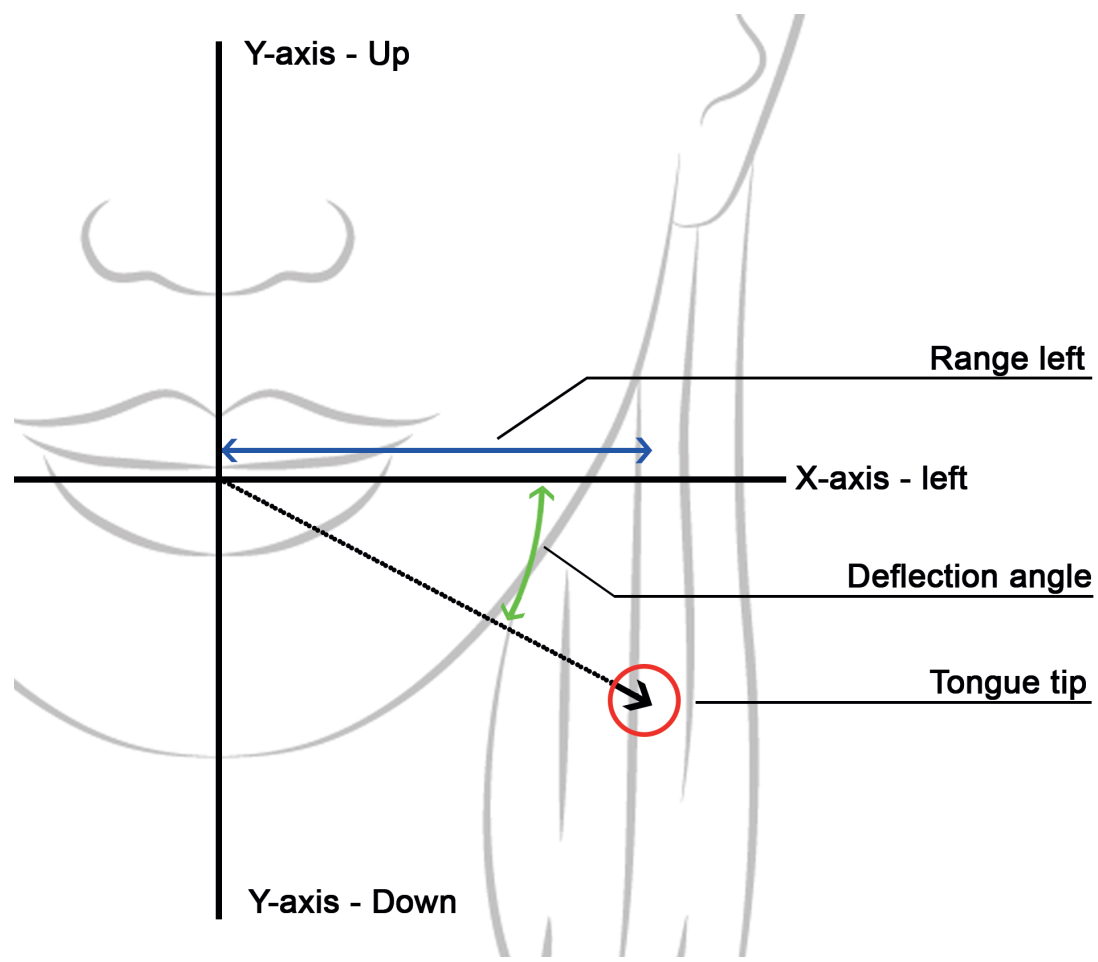

Figure 3.5 - Definition of the tongue's range (blue) and deflection angle (green) to the left side. The definition is based on the projection of the tongue tip (red circle) on the XY plane. The right side is a mirrored version of the left side. (background design by Freepik)

\section{Validation of the method}

The performance of this method depends on the variation that is induced by both the participant, the observer, the camera system and the software (Figure 3.1). The camera system was already validated in a previous study [15]. The root mean square error, for selecting a marker in 3D space, was $0.73 \mathrm{~mm}$, which was estimated using a leave-one-out method. 


\section{Intrarater and interrater reliability}

The observer plays a key role in some important parts of the process. It is essential that landmarks are selected and tracked in a reproducible way by the observers. Using the intra- and interrater reliability of two observers, expressed in the Intraclass Correlation Coefficient (ICC $2.1 \& 3.1$ ), we determined whether the manual interference in the tracking and processing steps is reliable. The ICC of the range and deflection angle indicators are measured using nine healthy participants. An ICC of more than 0.75 is considered an excellent agreement, and an ICC less than 0.4 a poor agreement. This was computed using SPSS (Version 25, IBM, 2018).

\section{Repeatability of the indicators with and without jaw compensation}

The main outcomes of our method are the range and deflection angle indicators. The repeatability of the indicators was assessed by measuring and processing a single healthy participant five times under the same conditions. The mean $(\mu)$ and the standard deviation $(\sigma)$ are calculated with and without jaw-movement compensation for every maneuver. To compare standard deviations between maneuvers the coefficient of variation $\left(c_{v}\right)$ is calculated for every indicator using:

$$
c_{v}=\frac{\mu}{\sigma}
$$

\section{Comparison with clinical expectations}

Since we only have healthy or post-treatment measurements, we can only focus on the asymmetry of a participant and the differences between groups. Therefore, the total range and deflection angle are calculated:

The total range from left to right:

$$
R_{\text {total }}=R_{r}+R_{l}
$$

The combined deflection angles from left and right:

$$
\varphi_{\text {total }}=\varphi_{r}+\varphi_{l}
$$

To emphasize the asymmetry, the difference between the ipsilateral and contralateral deflection angle ( $\varphi_{\text {ips }}$ and $\varphi_{\text {contra }}$ respectively) is calculated:

$$
\varphi_{\text {dif }}=\varphi_{\text {ips }}-\varphi_{\text {contra }}
$$

Where $\varphi_{i p s}$ equals either $\varphi_{r}$ or $\varphi_{l}$ depending on which side was affected.

The tumor stage (T-stage) is widely used as a parameter to categorize tumors and to differentiate patients regarding functional loss[33]. Therefore, asymmetry of the tongue range to the left and right will be compared between the two patient groups and tumor stage. Before the ranges can be compared, they have to be normalized: 


$$
R_{\text {ips,normed }}=\frac{R_{\text {ips }}}{R_{\text {total }}} R_{\text {contra,normed }}=\frac{R_{\text {contra }}}{R_{\text {total }}} \quad E q 3.5
$$

Where $R_{\text {ips }}$ equals either $R_{r}$ or $R_{l}$ depending on which side is affected. The ratio between normalized tongue ranges can be calculated by:

$$
R_{\text {dif }}=\frac{R_{\text {ips }}-R_{\text {contra }}}{R_{\text {total }}}
$$

\subsection{RESULTS}

Intrarater and interrater reliability of tracking and processing

The mean intra- and interrater reliability for the range and deflection angle are well above the ICC of 0.9 for all the measurements (see Table 3.1).

Table 3.1 - Intra- and interrater correlation coefficients for the range and deflection angle of nine healthy participants.

\begin{tabular}{rll}
\hline & Range & Deflection angle \\
\hline Intrarater (Jaw-comp) & $0.97(0.95-0.99)$ & $0.95(0.90-0.97)$ \\
ICC: 3,1 & & \\
\hline Interrater (Jaw-comp) & $0.96(0.93-0.98)$ & $0.92(0.84-0.96)$ \\
ICC: 2,1 & & \\
\hline
\end{tabular}

\section{Repeatability of the indicators with and without jaw compensation}

The mean coefficient of variation $\left(c_{v}\right)$ of the range and the $\sigma$ for both the range and deflection angle are shown in Figure 3.6. The measured ranges, excluding the up-maneuver, show a $c_{v}$ of about $7 \%$ ( $\sigma$ of $3.5 \mathrm{~mm}$ ) which, by adding the jaw-movement compensation, decreases to about 5,5\% ( $\sigma$ of $2.5 \mathrm{~mm}$ ). The up movement could not be reproduced as well as the other maneuvers; even though the $\sigma$ of the up maneuver is only about one $\mathrm{mm}$ larger than the other maneuvers, the range is very small, which results in a relatively large error ( $c_{v}$ of $\left.40 \%\right)$. The deflection angle also follows a similar pattern with the exception that the $s$ is slightly larger when using jaw-movement compensation. 

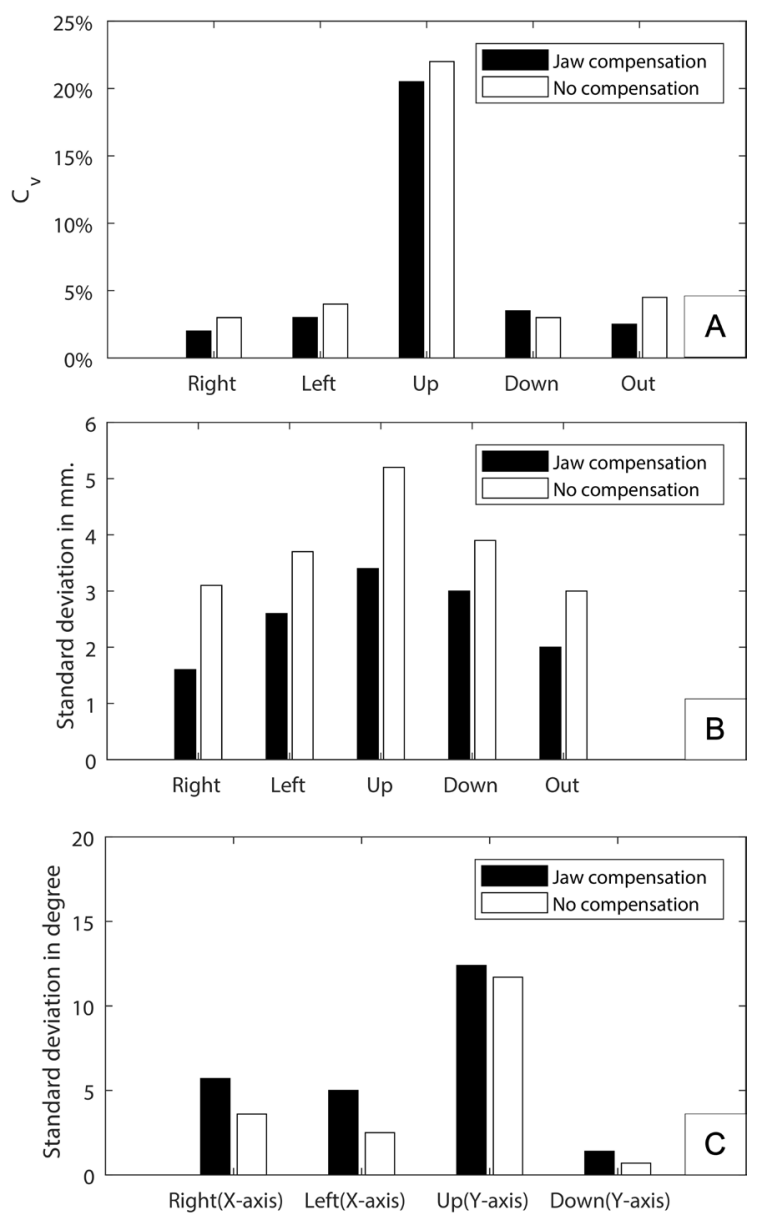

Figure 3.6 - Repeatability of the tongue's range and deflection angle indicators. Indicators with (black) and without jaw-movement compensation (white) are shown in bar plots in their specific unit measure. (a) the coefficient of variation $\left(C_{v}\right)$ of the tongue range in $\mathrm{mm}$. (b) standard deviation $(\sigma)$ of the tongue range in $\mathrm{mm}$. (c) standard deviation ( $\sigma$ ) of the tongue's deflection angle in angular degrees.

\section{Participant characteristics}

Two post-surgery patients were excluded. One patient could not understand the instructions and the other had undergone a re-resection that was not previously known by the researcher. One chemoradiation patient was excluded because the marker on the mental region was not visible in many of the video frames. No healthy participants were excluded.

Eventually, we included 19 healthy, 17 post-surgery and 18 post-chemoradiation participants. The characteristics of these groups are summarized in Table 3.2. 
Table 3.2 - Participant characteristics

\begin{tabular}{rllll}
\hline Group & $\begin{array}{l}\text { Median age } \\
\text { (Range) }\end{array}$ & $\begin{array}{l}\text { Tumor } \\
\text { location L/R }\end{array}$ & $\begin{array}{l}\text { Tumor stage T } \\
1 / 2 / 3 / 4\end{array}$ & $\begin{array}{l}\text { Time after } \\
\text { treatment in } \\
\text { weeks }\end{array}$ \\
\hline Healthy participants & $53(23-71)$ & - & - & - \\
\hline $\begin{array}{r}\text { Partial surgery } \\
\text { (mobile tongue) }\end{array}$ & $65(51-84)$ & $6 / 11$ & $7 / 9 / 1 / 0$ & $162(43-310)$ \\
\hline $\begin{array}{r}\text { Post-chemoradiation } \\
\text { (base of the tongue) }\end{array}$ & $65(48-79)$ & $11 / 7$ & $4 / 7 / 3 / 4$ & $182(21-706)$ \\
\hline
\end{tabular}

\section{Comparison with clinical expectations}

In Figure 3.7 the total range from left to right $\left(R_{\text {total }}\right)$ of all participants are expressed in bar plots. In this figure, the range is divided into bins of $10 \mathrm{~mm}$ for every participant group. The normalized counts per bin are expressed in percentages. The healthy participants clearly have the largest total range with the highest percentage around $85 \mathrm{~mm}$ whereas the post-surgery patients peak around $60 \mathrm{~mm}$. Post-chemoradiation is right in between those two groups.

We observe an inverse relationship if we compare the size of the total deflection angles $\left(\varphi_{\text {total }}\right)$ of the left and the right maneuver (Figure 3.8): the patients have more difficulties moving the tongue tip horizontally to the left or to the right than the healthy participants.

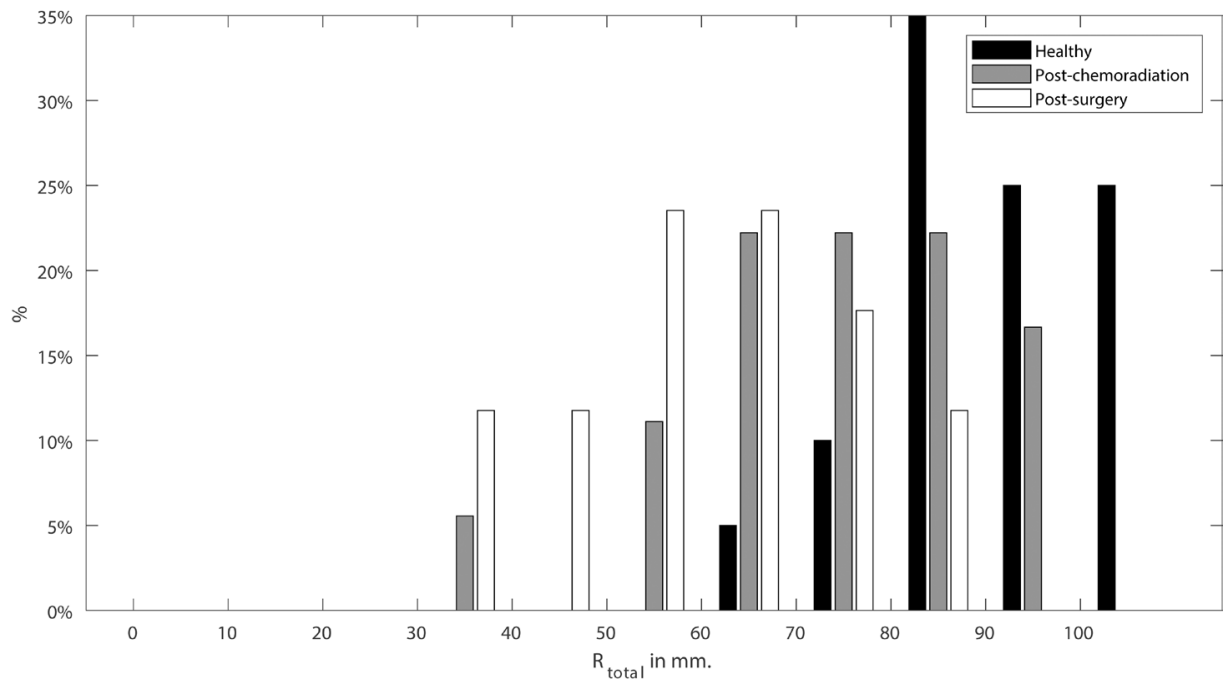

Figure 3.7 - The total range of the tongue $\left(R_{\text {total }}\right)$ for healthy, post-chemoradiation and post-surgery participants. The graph shows the percentage of participants that exhibit a certain range of tongue motion when moving from left to right within a specified interval. 


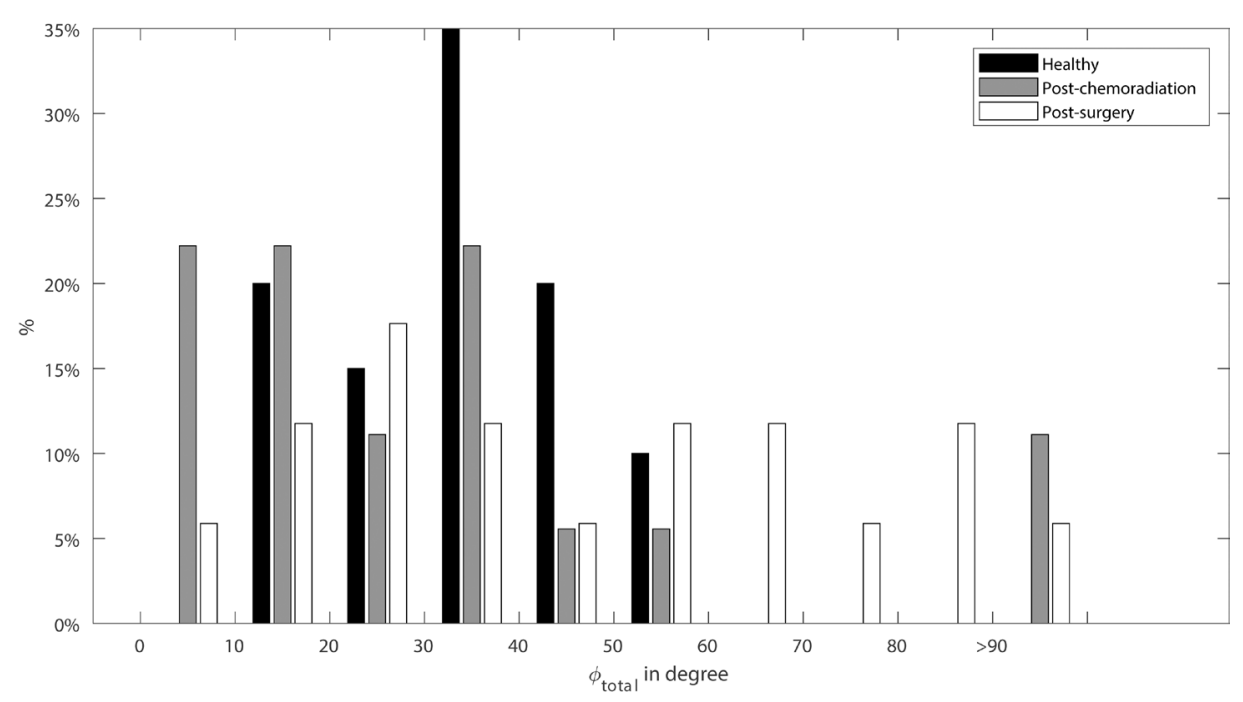

Figure 3.8 - The total deflection angle of the tongue $\left(\varphi_{\text {total }}\right)$ for healthy, post chemoradiation and surgery participants. The graph shows the percentage of participants that exhibit a total deflection angle for the left and right movement within a specified interval.

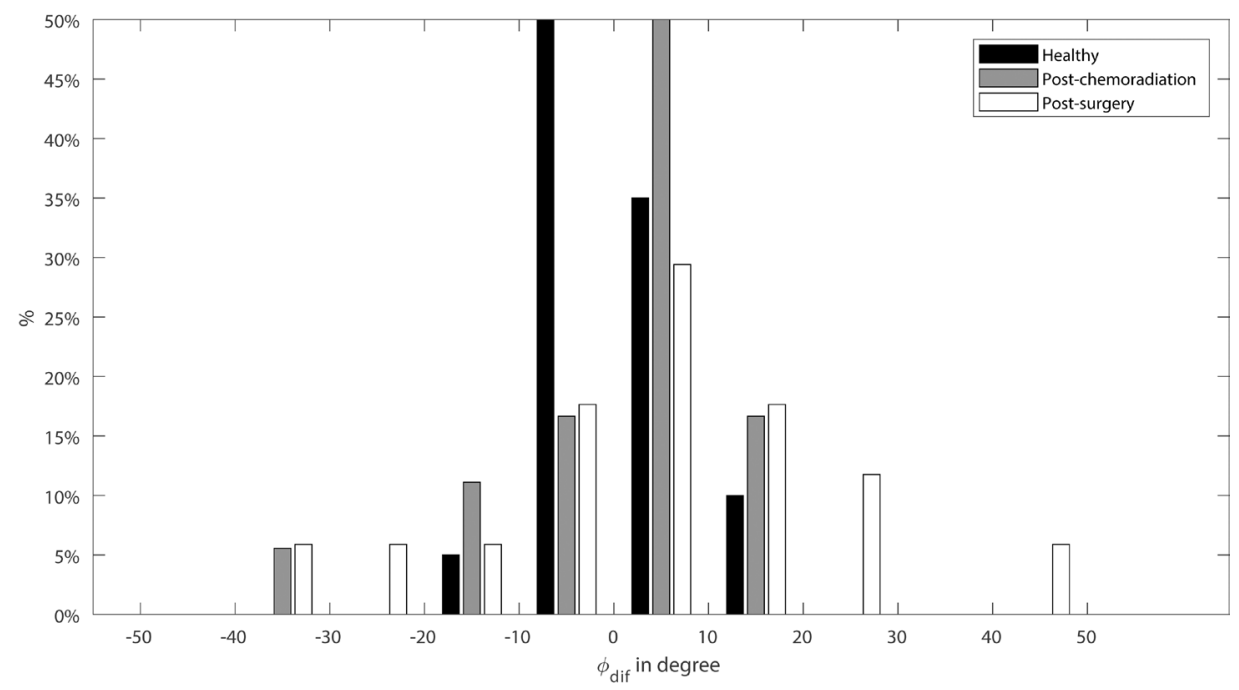

Figure 3.9 - The difference between Ipsilateral deflection angle and contralateral deflection angle $\left(\varphi_{\text {dif }}\right) . \varphi_{\text {dif }}$ is divided in bins of 10 angular degrees. For healthy participants, this is the difference between left and right deflection angle

Without preoperative measurements, the asymmetry between the ipsilateral and contralateral movements, $\varphi_{\text {dif }}$ and $R_{\text {dif }}$, is a more suitable measure for impairment than the total distance or angle. For comparison, the difference between left and right among healthy participants are shown in the upcoming 
figures. Figure 3.9 shows that most of the healthy participants express fairly symmetric behavior in moving tongue to the left and right at the same deflection angle. While most patients show this same symmetric behavior, about one-third of both patient groups show an asymmetry between the ipsilateral and contralateral angle $\left(\varphi_{\text {dif }}\right)$ of more than 10 degrees.

When looking at the difference between the normalized ipsilateral and contralateral range $\left(R_{\text {dif }}\right)$, we see a pattern that is comparable to the deflection angle (Figure 3.10). While most of the healthy participants only show a 0.1 difference on a -1 to 1 scale, the post-surgery patients can show differences of well over 0.2 . The impairments seen at both patient groups is predominantly to the contralateral side. The majority of patients do not show asymmetries that are distinguishable from the healthy participants.

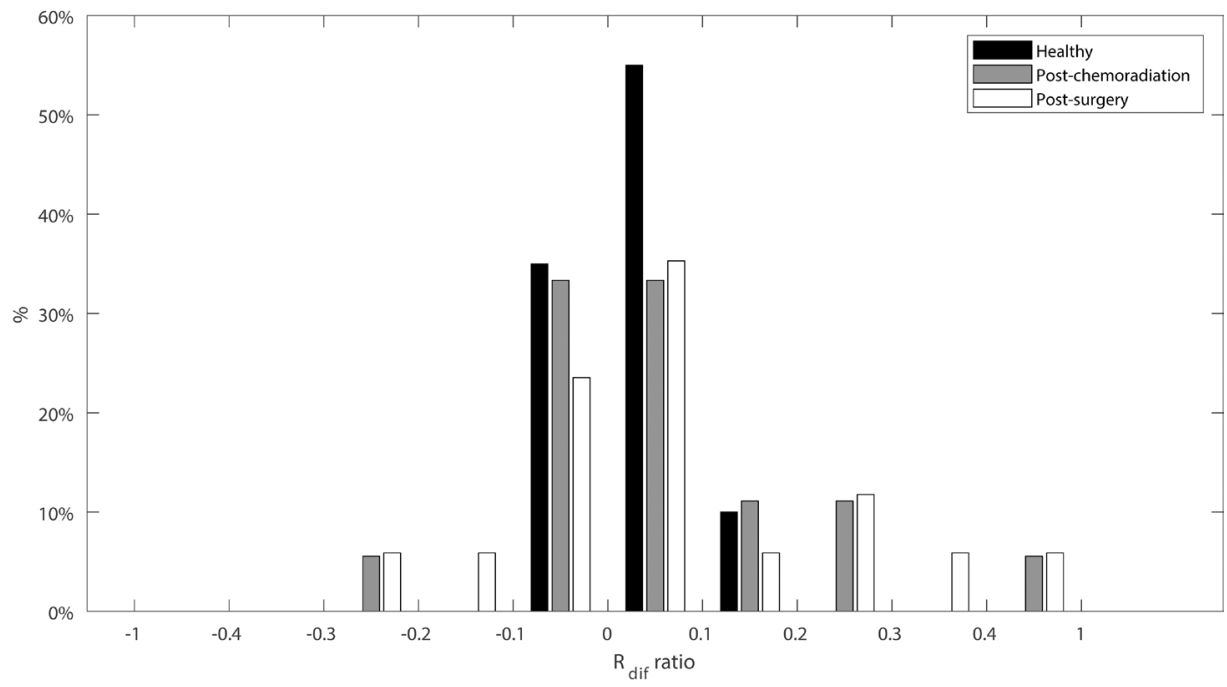

Figure 3.10 - Normalized difference between ipsilateral and contralateral range $\left(R_{\text {dif }}\right) \cdot R_{\text {dif }}$ is divided in bins of 0.1 on a -1 to 1 scale. For healthy participants, this is the difference between left and right deflection range.

To visualize the influence of tumor stage, the absolute differences between the normalized ipsilateral and the contralateral range $\left(R_{\text {dif }}\right)$ are shown in boxplots per T stage in Figure 3.11. The boxplot shows that the median of the difference does not differ much with increasing T-stage, but it shows an increased variability and an extended upper quartile of the boxplot that is especially predominant within the patient groups. Four patients with T4 tumors treated with chemoradiation showed no asymmetry. 


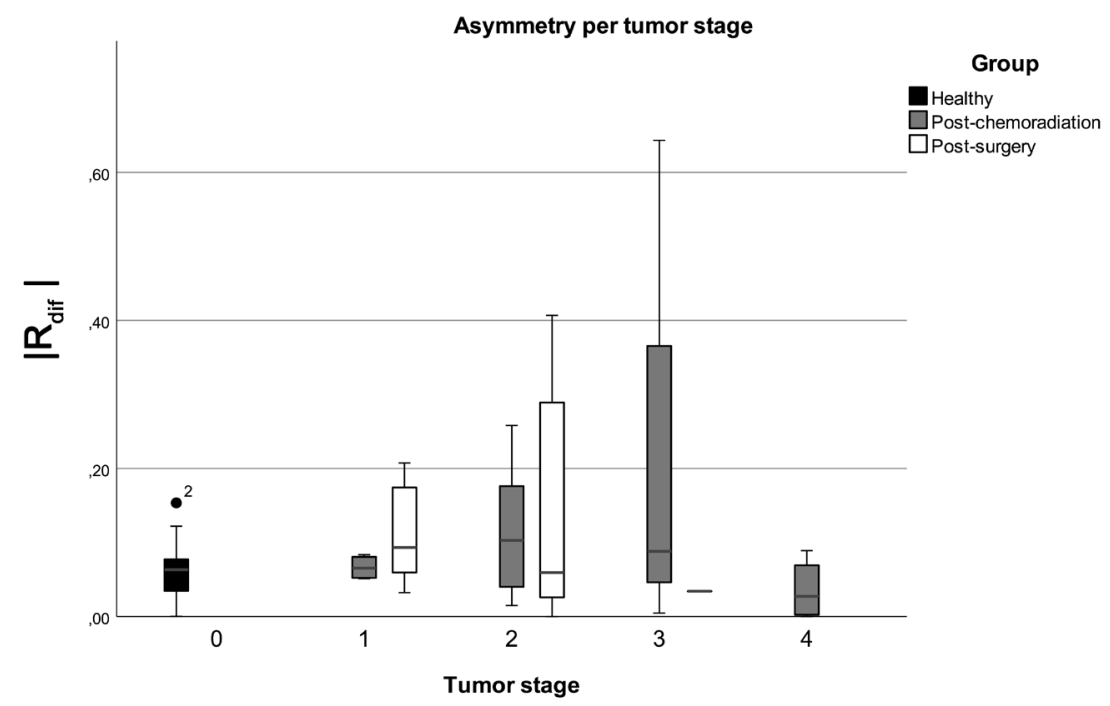

Figure 3.11 - Absolute normalized difference between ipsilateral and contralateral range $\left(R_{\text {dif }}\right)$ grouped in T-stage and participant group. The healthy participants group is black, post-chemoradiation is grey and post-surgery is white. The median is shown in dark-grey. There was only one T3 post-surgery patient which is shown as a small stripe. Created using SPSS statistics version 25 (IBM)

Figure 3.10 shows that in patients with asymmetrical movement the impairment is predominant on the contralateral side for a small number of patients. Since the range is now measured over an axis using a coordinate system, it is not possible to compare this data directly to our former study wherein only the Euclidean distances were compared. To show that the post-surgery group is comparable to our former study we calculated the Euclidean distances between the two central incisors and the tongue tip for both the ipsilateral and contralateral movement [15]. The results in Table 3.3 show that the mean Euclidian distance of our patient population is $2 \mathrm{~mm}$ smaller in both directions compared to the former study. While showing a significant difference between ipsilateral and contralateral, the Euclidian distance is still highly dependent on the initial pre-treatment ROM, and is therefore not as trustworthy as normalized measures.

Table 3.3 - Comparison of the mean Euclidian distance for ipsilateral and contralateral movement between the current study and the former study of van Dijk et al. [15].

\begin{tabular}{rll}
\hline Euclidian distance & Current study $(\mathrm{N}=17)$ & Former study $(\mathrm{N}=10)$ \\
\hline ipsilateral & $45.6 \mathrm{~mm}(7.9)$ & $47.5 \mathrm{~mm}(7.0)$ \\
\hline Contralateral & $40.8 \mathrm{~mm}(7.1)$ & $42.2 \mathrm{~mm}(5.8)$ \\
\hline $\mathrm{p}$-value & 0.016 & $<0.001$ \\
\hline
\end{tabular}




\subsection{DISCUSSION}

Tongue mobility impairment, for which ROM is an objective measure, has proven to be an important estimate for oral functions like speech [12]. A reliable method is therefore essential for both research and clinical practice. By elaborating on previous work, we created a method to track tongue tip trajectories and put these in perspective by introducing a coordinate system based on facial features [15]. This coordinate system enables localization of the tongue tip at every moment during tracking. By also compensating for head and jaw motion, this method proves to be a robust tool to measure the ROM more accessible and easier to use than other common techniques such as video fluoroscopy, CT, MRI, Ultrasound or EMA.

The range and deflection angle extracted from the 3D tongue tip trajectory showed excellent reliability with ICC's above the 0.9 , which is the same as or former study [15]. The repeatability experiment showed an overall small standard deviation of $2.5 \mathrm{~mm}$ to $3.5 \mathrm{~mm}$ for every movement, except for the upward movement. We experienced that the up movement was hard to perform and to reproduce for the healthy participants and impossible to perform for about half of the patients.

In addition, other indicators such as volumes, areas and more indicators from the $Y$ and $Z$ axis, which could be derived from the trajectories, were considered. While these could be of use when comparing the ROM of a single participant over time, it showed no additional value when comparing indicators between participants and patients using our current post-treatment dataset. Furthermore, measurement errors will rise exponentially when indicators are derived from multiple measurement points.

The addition of the jaw-movement compensation lowered the standard deviation for the range indicators and increased the standard deviation of the deflection angle by a small amount. While the differences in standard deviation are very small, the benefit of adding jaw-movement compensation is large, because some participants displayed more inherent jaw movements than others. This was mainly visible in compensatory behavior in healthy participants and patients with a very small ROM. A limitation of the jaw compensation was that some participants were able to cover their mandible area during the downward movement for which manual adjustment was needed.

In order to translate the tongue tip trajectory to interpretable results, we introduced a coordinate system. We choose to determine the coordinate system based on the maxillary incisors and the caruncles of the eyes because these points are fixed facial features that will retain the same location after tongue cancer treatment. This makes this system particularly suitable for repeated 
measurements of a single individual over time. The limitation, however, is that the orientation of the coordinate system will differ between individuals, as the eyes and the maxillary incisors are not aligned at the same angle. At this moment, there is no definitive solution to this problem. However, this will not influence the horizontal moments, which will, therefore, be more suitable to compare between individuals.

While large parts of the method are automated, some key parts of the method still depend on human interaction. During the acquisition, variation could be induced by misplacing the marker, insufficient instructions or non-compliance by the patient. In addition, the observer is responsible that the automatic tracking is performed properly. We found that in some cases the tracked 3D position drifted off over time due to small discrepancies between the tracked location in the three videos. A small error in one or two of the three videos can lead to a misinterpreted 3D location of the landmark, mainly in the transverse plane (or Z-axis). In a future release aimed at performing measurements in a clinical setting, an automated feedback system could inform the observer about significant back-projection errors. The system could also be improved by adding more cameras at different heights.

Tracking a single point in space is very quick, convenient and inexpensive. The measurements performed on patients were finished within minutes without any discomfort. This is an advantage in comparison to EMA which takes time to set up, is expensive, and uncomfortable for patients. However, measuring only one point has its limitations. The shape and position of the rest of the tongue remains unknown, which makes a ROM measurement at a single moment less useful speech and swallowing analysis. The ROMmeasurements are mostuseful when measured over a period of time to quantify the improvement or deterioration of tongue motion.

\section{Comparison with clinical expectations}

Tongue movement varies greatly between participants. In our study, the range from left to right ( $R_{\text {total }}$ ) within healthy participants varies from $60 \mathrm{~mm}$ to over $100 \mathrm{~mm}$ (Figure 3.7). Because of this variation, it is not possible to distinguish an individual patient from a healthy participant purely on the range or deflection angle; but as a group, they are clearly distinguishable in Figure 3.7 and Figure 3.8. We can, therefore, assume that in general, a post-surgical patient not only has a smaller range, which was expected based on previous research but also has a larger deflection angle compared to healthy participants. The post-chemoradiation patients are in between those two groups, which was expected based on the fact that no tissue is removed and that these tumors involve the base of the tongue, that more often results in problems with swallowing rather than problems with lateral movement of the mobile tongue [3,11]. 
However, using only post-treatment data, a fair comparison was only possible by comparing the contralateral and ipsilateral properties between participants. Based on previous studies we hypothesized that impaired motion to the contralateral side would be predominant [15]. In Figure 3.10 the largest impairments are seen when moving to the contralateral side, however, this is not always the case. Only four post-surgery and three post-chemoradiation participants showed a serious contralateral impairment. No dominant impairment to a side is visible when looking at the difference between deviation angles $\left(\varphi_{\text {dif }}\right)$ in Figure 3.9. This is in line with studies that show that the side affected by the defect does not matter or not always lead to lateralization problems[6,13]. However, when calculating the Euclidean distance for the left to right movement, a significant contralateral impairment, comparable with our previous study, is visible (Table 3.3) [15]. We hypothesize that the combination of the contralateral range and angle result in a mean Euclidean contralateral distance that is significantly different from the ipsilateral distance. However, based on the variation seen in the other Figures (Figure 3.7, Figure 3.8 and Figure 3.9) we assume that this is the case for only some post-surgery patients.

It is clear that some parameters are responsible for the large variation between the three groups. The upper quartile in Figure 3.9 reflects that a substantial amount of patients have an increased asymmetric movement of the tongue with increasing T-stage, which was expected based on literature [33,34]. However, while the variation in asymmetry increases with T-stage and type of treatment, the median does not, which is largely in line with the rest of the results. The same can be found in other literature such as Zuydam et al. [8] where speech scores after surgery are overlapping between T-stages or did not yield significant correlation with speech function $[8,10]$. Furthermore, it is shown in previous literature that post-operative impairment not only depends on the size and location of the treatment but also on the amount of scar tissue and compensatory tongue motion patterns $[12,14]$. In the case of a T4, the tumor usually also involves other tissues in the oropharynx where we cannot account for. The inclusion of these parameters would require a larger study population and would be outside the scope of this paper. Furthermore, data about the resection volume and location that were collected retrospectively are rarely precise. Future studies should focus on collection of detailed information on size, tissue-, muscle- and innervationproperties of the tongue. To analyze the effects on ROM in detail, location and size of the treated area of the tongue in patients should be included as well. Also, pre- and post-treatment measures of swallowing and assessment of speech quality and intelligibility can be used to assess if ROM is a valuable tool for the prediction of function loss. 


\subsection{CONCLUSION}

We elaborated on previous work of van Dijk et al. [15] to introduce an improved reliable and reproducible method to measure the ROM and to quantify for motion impairments as a fast, secure, and accessible alternative for classical imaging techniques and EMA. Using this method, the exact location of the tongue tip can be tracked throughout different tongue maneuvers, while also compensating for head and jaw movement, and thus extending the possibilities of ROM measurements. This way of objectively obtaining the ROM of the tongue tip is essential for methods aimed at predicting treatment outcome, such as biomechanical prediction models, as an addition to the shared decision making in treatment choices. Moreover, it would also greatly improve the objectivity of determining progress during logopedic treatment and rehabilitation in which improvement lingual mobility is the primary focus [16].

With this improved method, we explored the various indicators from which tongue range and deflection angle could be explored and validated using our dataset. From the post-surgery and post-chemoradiation patients, only a small part showed asymmetrical movements, which could not always be explained by T-stage or the side of treatment alone. Future studies should focus on measuring ROM in patients with oral cancer pre- and post-treatment in combination with functional measures and detailed characteristics of the treatment to show if a change in ROM is predictive for functional loss. 


\subsection{REFERENCES}

1. UK Cancer Research. Head and neck cancers incidence statistics [Internet]. 2019 [cited 26 Apr 2019]. Available: https://www.cancerresearchuk.org/healthprofessional/cancer-statistics/statistics-by-cancer-type/head-and-neck-cancers/ incidence\#heading-Four

2. Garnaes E, Kiss K, Andersen L, Therkildsen MH, Franzmann MB, Filtenborg-Barnkob B, et al. Increasing incidence of base of tongue cancers from 2000 to 2010 due to HPV: The largest demographic study of 210 Danish patients. Br J Cancer. Nature Publishing Group; 2015;113: 131-134. doi:10.1038/bjc.2015.198

3. Sessions DG, Spector GJ, Lenox J, Haughey B, Chao C, Marks J. Analysis of treatment results for oral tongue cancer. Laryngoscope. 2002;112: 616-25. doi:10.1097/00005537-200204000-00005

4. Van Der Molen L, Van Rossum MA, Jacobi I, Van Son RJJH, Smeele LE, Rasch CRN, et al. Pre- and posttreatment voice and speech outcomes in patients with advanced head and neck cancer treated with chemoradiotherapy: Expert listeners' and patient's perception. J Voice. Elsevier Ltd; 2012;26: 664.e25-664.e33. doi:10.1016/j.jvoice.2011.08.016

5. Kreeft AM, Van Der Molen L, Hilgers FJ, Balm AJ. Speech and swallowing after surgical treatment of advanced oral and oropharyngeal carcinoma: A systematic review of the literature. Eur Arch Oto-Rhino-Laryngology. Springer; 2009;266: 1687-1698. doi:10.1007/s00405-009-1089-2

6. Bressmann T, Ackloo E, Heng C Le, Irish JC. Quantitative three-dimensional ultrasound imaging of partially resected tongues. Otolaryngol - Head Neck Surg. 2007;136: 799805. doi:10.1016/j.otohns.2006.11.022

7. Stone M, Langguth JM, Woo J, Chen H, Prince JL. Tongue motion patterns in postglossectomy and typical speakers: a principal components analysis. J Speech Lang Hear Res. United States; 2014;57: 707-717. doi:10.1044/1092-4388(2013/13-0085)

8. Zuydam AC, Lowe D, Brown JS, Vaughan ED, Rogers SN. Predictors of speech and swallowing function following primary surgery for oral and oropharyngeal cancer. Clin Otolaryngol. 2005;30: 428-437. doi:10.1111/j.1365-2273.2005.01061.x

9. Suarez-Cunqueiro MM, Schramm A, Schoen R, Seoane-Lestón J, Otero-Cepeda XL, Bormann $\mathrm{KH}$, et al. Speech and swallowing impairment after treatment for oral and oropharyngeal cancer. Arch Otolaryngol - Head Neck Surg. 2008;134: 1299-1304. doi:10.1001/archotol.134.12.1299

10. Lee DY, Ryu YJ, Hah JH, Kwon TK, Sung MW, Kim KH. Long-term subjective tongue function after partial glossectomy. J Oral Rehabil. 2014;41: 754-758. doi:10.1111/ joor.12193

11. Rosebush MS, Rao SK, Samant S, Gu W, Handorf CR, Pfeffer LM, et al. Oral cancer: enduring characteristics and emerging trends. J Tenn Dent Assoc. United States; 2011;91: 24-7.

12. Matsui Y, Ohno K, Yamashita Y, Takahashi K. Factors influencing postoperative speech function of tongue cancer patients following reconstruction with fasciocutaneous/ myocutaneous flaps-a multicenter study. Int J Oral Maxillofac Surg. 2007;36: 601609. doi:10.1016/j.ijom.2007.01.014

13. Konstantinović VS, Dimić ND. Articulatory function and tongue mobility after surgery followed by radiotherapy for tongue and floor of the mouth cancer patients. Br J Plast Surg. 1998;51: 589-593. doi:10.1054/bjps.1998.0202 
14. Bressmann T, Sader R, Whitehill TL, Samman N. Consonant intelligibility and tongue motility in patients with partial glossectomy. J Oral Maxillofac Surg. 2004;62: 298-303. doi:10.1016/j.joms.2003.04.017

15. van Dijk S, van Alphen MJA, Jacobi I, Smeele LE, van der Heijden F, Balm AJM. A New Accurate 3D Measurement Tool to Assess the Range of Motion of the Tongue in Oral Cancer Patients: A Standardized Model. Dysphagia. Springer US; 2016;31: 97-103. doi:10.1007/s00455-015-9665-7

16. Husaini H, Krisciunas GP, Langmore S, Mojica JK, Urken ML, Jacobson AS, et al. A survey of variables used by speech-language pathologists to assess function and predict functional recovery in oral cancer patients. Dysphagia. 2014;29:376-386. doi:10.1007/ s00455-014-9520-2

17. Kappert KDR, van Alphen MJA, van Dijk S, Smeele LE, Balm AJM, van der Heijden F. An interactive surgical simulation tool to assess the consequences of a partial glossectomy on a biomechanical model of the tongue. Comput Methods Biomech Biomed Engin. 2019;22: 827-839. doi:10.1080/10255842.2019.1599362

18. Van Alphen MJA, Kreeft AM, Van Der Heijden F, Smeele LE, Balm AJM. Towards virtual surgery in oral cancer to predict postoperative oral functions preoperatively. Br J Oral Maxillofac Surg. British Association of Oral and Maxillofacial Surgeons; 2013;51: 747 751. doi:10.1016/j.bjoms.2013.06.012

19. Buchaillard S, Brix M, Perrier P, Payan Y. Simulations of the consequences of tongue surgery on tongue mobility: Implications for speech production in post-surgery conditions. Int J Med Robot ComputAssist Surg. 2007;3: 252-261. doi:10.1002/rcs.142

20. Xu K, Yang Y, Stone M, Jaumard-Hakoun A, Leboullenger C, Dreyfus G, et al. Robust contour tracking in ultrasound tongue image sequences. Clin Linguist Phonetics. Informa Healthcare; 2016;30: 313-327. doi:10.3109/02699206.2015.1110714

21. Masterson JA, Li SR, Woeste HM, Dugan S, Mahalingam N, Boyce SE, et al. A real-time, automated tongue tracking method for ultrasound biofeedback in speech therapy. J Acoust Soc Am. Acoustical Society of America;2019;145:1794.doi:10.1121/1.5101563

22. Blyth KM, Mccabe P, Madill C, Ballard KJ. Ultrasound visual feedback in articulation therapy following partial glossectomy. J Commun Disord. Elsevier Inc.; 2016;61: 1-15. doi:10.1016/j.jcomdis.2016.02.004

23. Xing F, Woo J, Lee J, Murano EZ, Stone M, Prince JL. Analysis of 3-D Tongue Motion From Tagged and Cine Magnetic Resonance Images. J Speech, Lang Hear Res. 2016;59: 468-479. doi:10.1044/2016_jslhr-s-14-0155

24. Ramanarayanan V, Tilsen S, Proctor M, Töger J, Goldstein L, Nayak KS, et al. Analysis of speech production real-time MRI. Comput Speech Lang. Elsevier Ltd; 2018;52: 1-22. doi:10.1016/j.csl.2018.04.002

25. Lander-Portnoy M, Goldstein L, Narayanan SS. Using real time magnetic resonance imaging to measure changes in articulatory behavior due to partial glossectomy. J Acoust Soc Am. Acoustical Society of America; 2017;142:2641-2642. doi:10.1121/1.5014684

26. Ha J, Sung I, Son J, Stone M, Ord R, Cho Y. Analysis of speech and tongue motion in normal and post-glossectomy speaker using cine MRI. J Appl Oral Sci. 2016;24: 472480. doi:10.1590/1678-775720150421

27. Perkell JS, Cohen MH, Svirsky M a, Matthies ML, Garabieta I, Jackson MTT. Electromagnetic midsagittal articulometer systems for transducing speech articulatory movements. J Acoust Soc Am. 1992;92: 3078-3096. doi:10.1121/1.404204 
28. Kaburagi T, Honda M. Electromagnetic articulograph based on a nonparametric representation of the magnetic field. J Acoust Soc Am. United States, United States; 2002;111: 1414-1421. doi:10.1121/1.1445785

29. Kuruvilla M, Murdoch B, Goozèe J. Electromagnetic articulography assessment of articulatory function in adults with dysarthria following traumatic brain injury. Brain Inj. 2007;21: 601-613. doi:10.1080/02699050701427525

30. Fuentes R, Arias A, Lezcano MF, Saravia D, Kuramochi G, Dias FJ. Systematic Standardized and Individualized Assessment of Masticatory Cycles Using Electromagnetic 3D Articulography and Computer Scripts. Biomed Res Int. 2017;2017: 1-9. doi:10.1155/2017/7134389

31. KatzWF, Mehta S, Wood M, Wang J. Using electromagnetic articulography with a tongue lateral sensor to discriminate manner of articulation. J Acoust Soc Am. 2017;141: EL57EL63. doi:10.1121/1.4973907

32. Haworth B, Kearney E, Faloutsos P, Baljko M, Yunusova Y. Electromagnetic articulography (EMA) for real-time feedback application: computational techniques. Comput Methods Biomech Biomed Eng Imaging Vis. Taylor \& Francis; 2018;1163: 1-8. doi:10.1080/216 81163.2018.1434423

33. Borggreven PA, Verdonck-De Leeuw I, Langendijk JA, Doornaert P, Koster MN, De Bree $\mathrm{R}$, et al. Speech outcome after surgical treatment for oral and oropharyngeal cancer: A longitudinal assessment of patients reconstructed by a microvascular flap. Head Neck. 2005;27: 785-793. doi:10.1002/hed.20236

34. Lazarus CL, Husaini H, Jacobson AS, Mojica JK, Buchbinder D, Okay D, etal. Development of a new lingual range-of-motion assessment scale: Normative data in surgically treated oral cancer patients. Dysphagia. 2014;29: 489-499. doi:10.1007/s00455-014-9534-9 



\title{
FIVE SPECIFIC TONGUE MOVEMENTS IN A HEALTHY POPULATION
}

\author{
K.D.R. Kappert \\ S. van Dijk \\ D. Wellenstein \\ M.J.A. van Alphen \\ R. van Son \\ L.E. Smeele \\ A.J.M. Balm
}

This chapter was published in Dysphagia:

Kappert KDR, van Dijk S, Wellenstein D, van Alphen MJA, van Son RJJH, Smeele LE, Balm AJM. Five Specific Tongue Movements in a Healthy Population. Dysphagia. 2020; doi:10.1007/s00455-020-10195-y 


\section{ABSTRACT}

The importance of tongue mobility on speech, oral food transport, and swallowing is well recognized. However, whether the individual tongue mobility influences postoperative function in oral cancer treatment remains to be elucidated. This study assesses the ability to perform five tongue movements as rolling, twisting (two sides), folding, and the 'cloverleaf' in a healthy population. Because a tumor in oral cancer patients often restricts the mobility of the tongue, it might be helpful to know if it is possible to recall any of those movements without demonstrating it.

Two observers asked 387 Dutch healthy adults if they could perform one of the five specific tongue movements and were subsequently asked to demonstrate the five movements.

The distribution in the Dutch population is: rolling: $83.7 \%$, cloverleaf: $14.7 \%$, folding: $27.5 \%$, twisting left: $36.1 \%$ and twisting right: $35.6 \%$. The percentage of people that can fold their tongue is almost ten times higher (3\% versus 27.5\%) than in previous research, and it was found that the ability to roll the tongue is not a prerequisite for folding of the tongue. A relationship between gender or right-handedness and the ability to perform certain tongue movements could not be found. Of the participants, $9.9 \%$ and $13.1 \%$ incorrectly assumed that they could demonstrate tongue rolling and cloverleaf. Tongue folding and twisting (left or right) were incorrectly assumed in $36.9 \%, 24.1 \%$, and $25.4 \%$ of the cases. Rolling and cloverleaf are preferred for future prediction models because these movements are easy to recall without demonstrating. 


\section{$4.1 \quad$ INTRODUCTION}

The importance of tongue mobility on speech, oral food transport, and swallowing is well recognized. Particularly in oral cancer treatment, the prediction of function loss deserves a lot of attention [1-5]. However, whether the individual tongue mobility influences postoperative function remains to be elucidated [6].

The first article about tongue movements dates back to 1940 and since then, limited data have been published. Six specific tongue movements have been described thus far, being: Rolling, Folding, Twisting (2 sides), Cloverleaf, and the Pointing tongue [7].

The percentage of people who can roll the tongue varies from $60 \%$ to $80 \%$ [815 ] and the average percentage of tongue folding lies between $1.5 \%$ and $3 \%$ $[10,16,17]$. The capability of tongue folding is believed to depend on the presence of the tongue rolling gene $[9,10,18]$, but the genetic evidence is doubtful for other movements since little has been published about other features of the tongue, such as the 'cloverleaf' tongue [10,12].

Most of the published data showed no differences for tongue rolling, tongue folding, and the 'cloverleaf' tongue between sexes [8,10,11,13,14,16]. One study showed a sex difference in the ability to fold and roll the tongue in favor of women [12]. Furthermore, one study showed that right-handed women were able to roll the tongue significantly more compared to right-handed men [15].

The main purpose of this study is to assess the ability to perform five of the before mentioned tongue movements as rolling, twisting (two sides), folding, and the 'cloverleaf' in a healthy population (Figure 4.1). The sixth skill, a pointing tongue, is omitted because the authors thought this movement is too difficult to judge objectively.

Furthermore, we want to investigate if people are aware if they possess the ability to perform any of the specific tongue movements. Some patients do better after partial glossectomy than others, but the reason for this is not always clear.

The muscle topology of the tongue is overall the same among humans, but the innervation is known to differ between individuals [19,20], which might contribute to the individual versatility of this organ. Pretreatment exercises can influence the postoperative strength and function, and in general, it is assumed that a better preoperative condition also benefits postoperative compensatory movements $[21,22]$. It is hypothesized that genetically determined versatility of the tongue could be indicative of the rehabilitation results by the residual mobile capacity. 
Currently, there's no literature available suggesting any correlation. However, it is logical to assume that the ability to perform any of the aforementioned specific tongue movements are a derivative of neural control and mobility. Therefore it could be a decisive factor for the prediction of expected postoperative tongue function. Since tongue cancer interferes with the mobility of the tongue, it is a prerequisite that patients can reliably recall if they mastered the task of movement before the tumor occurred.

In summary, the study aims to assess the distribution of five specific tongue movements in a healthy population. Secondly, the awareness of these abilities will be assessed and possible differences in gender and handedness will be evaluated.

\subsection{METHOD}

Two observers performed a survey of Dutch healthy volunteers (18+) recruited at common gathering spots in our institute for four consecutive days. During this period, 387 surveys were conducted, which was enough to reach $95 \%$ confidence intervals of less than $\pm 5 \%$ for all movements considering the previous results of LU [7]. All volunteers were employees of different departments and a variety of functions. Characteristics that were taken into evaluation were gender, native language, and left- or right-handedness. Exclusion criteria were: (history of) a tumor in the oral cavity and any (neuromuscular) condition that might influence the mobility of the tongue. The survey contained two parts. In the first part participants answered two questions I: "Do you know any of the five movements as tongue rolling, folding, twisting, and the 'cloverleaf' tongue?". A picture of the five tongue movements was shown during the question (Figure 4.1). Question II: "Do you possess any of the tongue mobility features, and when YES; which ones?". During the second part, participants showed if they were able to demonstrate the five movements.

Each participant was rated by one of the two raters according to the pictures in Figure 4.1. A participant was either scored as capable (1) or as incapable (0) for each of the five tongue movements. Each participant could make three attempts to perform any of the movements. It was allowed to use a mirror during the assessment. When participants accomplished only a part of the movement this was scored as 0: incapable.

Participants were recruited on the institute premises and consisted of employees. All procedures performed in studies involving human participants were in accordance with the ethical standards of the medical ethical committee of the Netherlands Cancer Institute and with the 1964 Helsinki declaration and its later 
amendments or comparable ethical standards. Written Informed consent was obtained from all individual participants included in the study.
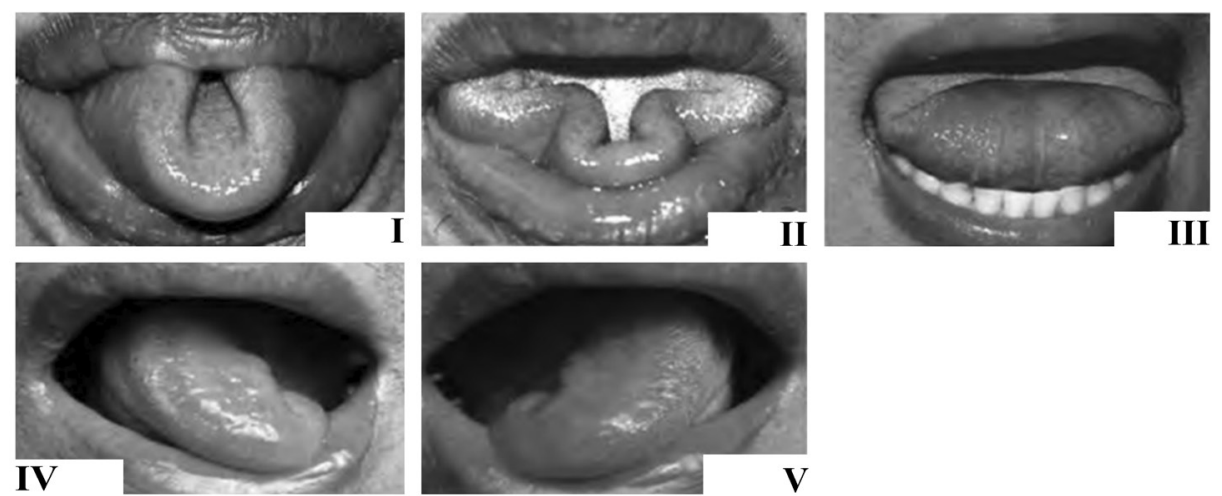

II

Figure 4.1 - An image with the five specific movements shown to participants just before they are asked about their ability to perform one of the movements. I: rolling, II: cloverleaf, III: folding, IV: twisting left, V: twisting right, folding, III. This image was adapted from Lu [7].

\section{Statistical Analysis}

ThePearsonChi-squaretestwasused toassessthecorrelation between participant characteristics and the ability to perform one of the five tongue movements. The same test was used to assess if there is was a significant difference between the two raters. Because all participants were only rated once, no interrater reliability assessment was performed. For all primary outcome measures the confidence interval for proportions was calculated using the following formula [23]:

$$
\hat{P} \pm Z \sqrt{\frac{\hat{P}(1-\hat{P})}{n}}
$$
With: $\hat{P}=\frac{n r \text { of cases }}{\text { sample size }(n)}$ and $Z=Z_{\frac{\alpha}{2}}=1.96$ using the Z-table (95\% confidence
interval)

\subsection{RESULTS:}

\section{Participant characteristics}

From the 387 participants on whom the survey was performed, 13 were excluded because of incomplete data, resulting in 374 included participants. The mean age was 37 years, ranging from 19 to 64 years old, $61 \%$ were females, and $85.8 \%$ were right-handed. This survey was performed on employees of the Netherlands Cancer Institute and $92.5 \%$ spoke Dutch as their native language. 
The Pearson Chi-Square did not show any association between handedness or gender and the ability to perform a certain movement $(P>0.05$, Table 4.1).

Table 4.1 - The Pearson chi-square test and significance value for each of the movements vs handedness or gender.

\begin{tabular}{lllll}
\hline & \multicolumn{2}{c}{ Pearson } & Chi-Square & \multicolumn{2}{c}{ Asymptotic Significance (2-sided) } \\
\cline { 2 - 5 } & \multicolumn{1}{c}{ Handedness } & \multicolumn{1}{c}{ Gender } & Handedness & Gender \\
\hline I: Rolling & 0.894 & 0.567 & 0.334 & 0.753 \\
\hline II : Cloverleaf & 0.80 & 1.018 & 0.777 & 0.601 \\
\hline III: Folding & 0.18 & 2.847 & 0.893 & 0.241 \\
\hline IV: Twisting (left) & 0.10 & 1.595 & 0.921 & 0.451 \\
\hline V: Twisting (Right) & 0.035 & 1.688 & 0.852 & 0.430 \\
\hline
\end{tabular}

\section{Agreement researchers}

A chi-square test was performed for the two raters and every type of tongue movement (Table 4.2). The test showed no association with the observer and the outcome $(P>0.05)$.

Table 4.2 - Pearson chi-square test and significance value to test if the difference between raters is significant.

\begin{tabular}{lcc}
\hline & Pearson Chi-Square & Asymptotic Significance (2-sided) \\
\hline I: Rolling & 2.354 & 0.125 \\
\hline II : Cloverleaf & 3.061 & 0.080 \\
\hline III: Folding & 3.566 & 0.059 \\
\hline IV: Twisting (left) & 0.877 & 0.349 \\
\hline V: Twisting (Right) & 0.079 & 0.778 \\
\hline
\end{tabular}

Table 4.3a shows the percentage of the participants that can and think they can performacertain type of movement. Tonguerolling is theeasiest tongue movement which can be done by $83.7 \%$ of the participants, and only $9.9 \%$ misjudge their ability to do so (Table 4.3b). The cloverleaf is the hardest movement to carry out; only $14.7 \%$ of the participants could do so. The misjudgment for this movement is relatively low, as can be seen in Table $4.3 \mathrm{~b}$. The $95 \%$ confidence intervals remained $< \pm 5 \%$ for all movements. Table $4.3 \mathrm{~b}$ also shows conditional probabilities, for example, the percentage from the participants that think they can fold, but cannot (3b, type1). Almost half of the participants think they can make a folding or a left/ right twist with their tongue, while the actual success rate is about $8-16 \%$ less. The misjudgment (Table $4.3 \mathrm{~b}$ ) of these three movements varies between $24 \%$ and $37 \%$. Folding is the most overestimated movement since $56.3 \%$ of the participants 
(Table 4.3b, type 1) failed. As a result of smaller subgroups, some combinations of misjudgments of movements have $95 \%$ confidence intervals $> \pm 5 \%$.

Table 4.3 - Percentages and 95\% confidence interval of the participants who a) can / think they can b) judged their ability wrong by type 1: think they can but actually cannot and type 2: think they cannot but actually can.

\begin{tabular}{llllll}
\hline $\begin{array}{l}\text { a) } \\
\text { Abilities }\end{array}$ & I: Rolling & II: Cloverleaf & III: Folding & $\begin{array}{l}\text { IV: Twisting } \\
\text { (left) }\end{array}$ & $\begin{array}{l}\text { V: Twisting } \\
\text { (Right) }\end{array}$ \\
\hline Can & $83.7 \% \pm 3.7$ & $14.7 \% \pm 3.5$ & $27.5 \% \pm 4.5$ & $36.1 \% \pm 4.9$ & $35.6 \% \pm 4.9$ \\
\hline Think & $77.0 \% \pm 4.3$ & $5.3 \% \pm 2.28$ & $43.6 \% \pm 5$ & $43.6 \% \pm 5$ & $44.9 \% \pm 5$ \\
\hline $\begin{array}{l}\text { b) } \\
\text { Wrong } \\
\text { judgment }\end{array}$ & $9.9 \% \pm 3.0$ & $13.1 \% \pm 3.4$ & $36.9 \% \pm 4.9$ & $24.1 \% \pm 4.3$ & $25.4 \% \pm 4.4$ \\
\hline $\begin{array}{l}\text { Type 1: } \\
\text { Think yes but } \\
\text { cannot }\end{array}$ & $2.1 \% \pm 1.7$ & $35.0 \% \pm 21.0$ & $60.7 \% \pm 7.5$ & $36.4 \% \pm 7.4$ & $38.7 \% \pm 7.4$ \\
\hline $\begin{array}{l}\text { Type 2: } \\
\text { Think not but can }\end{array}$ & $36.0 \% \pm 10.2$ & $11.9 \% \pm 3.4$ & $18.5 \% \pm 5.2$ & $14.7 \% \pm 4.8$ & $14.6 \% \pm 4.8$ \\
\hline
\end{tabular}

Only $32.5 \%$ of the participants can perform more than two separate movements with their tongue (Table 4.4). Participants who think they can perform two or three tongue movements overestimate their ability to carry out complex movements by about $8 \%$. Participants who can perform all of the five movements generally underestimate their ability to do so by about $3 \%$. While no correlation could be found between gender and the ability to perform a certain movement, there appears to be aslight advantage for women in the number of movements that can be performed. There are more women than men that can perform 2 and 3 movements. There are slightly more men that can perform all five movements. There is no indication that men overestimate there ability more than women and visa-versa.

Table 4.4 - Percentages of female, male and total number of participants who can perform and think they can perform at least a number of ' $x$ ' movements

\begin{tabular}{|c|c|c|c|c|c|c|c|}
\hline & & None & 1 or more & 2 or more & 3 or more & 4 or more & 5 \\
\hline \multirow{2}{*}{ 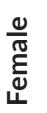 } & Can & $11.1 \%$ & $88.9 \%$ & $59.3 \%$ & $35.4 \%$ & $16.4 \%$ & $4.0 \%$ \\
\hline & Think & $9.7 \%$ & $90.3 \%$ & $67.0 \%$ & $42.3 \%$ & $17.2 \%$ & $1.8 \%$ \\
\hline \multirow{2}{*}{$\frac{0}{\frac{0}{\pi}}$} & Can & $12.4 \%$ & $87.6 \%$ & $47.6 \%$ & $28.3 \%$ & $16.6 \%$ & $6.2 \%$ \\
\hline & Think & $11.0 \%$ & $89.0 \%$ & $58.2 \%$ & $38.4 \%$ & $21.2 \%$ & $2.1 \%$ \\
\hline \multirow{2}{*}{$\begin{array}{l}\bar{\pi} \\
\stackrel{0}{0} \\
\qquad\end{array}$} & Can & $11.6 \%$ & $88.4 \%$ & $54.8 \%$ & $32.5 \%$ & $16.4 \%$ & $4.8 \%$ \\
\hline & Think & $10.2 \%$ & $89.8 \%$ & $63.4 \%$ & $40.6 \%$ & $18.7 \%$ & $1.9 \%$ \\
\hline
\end{tabular}


Table 4.5 shows the percentage of participants that can perform a specific movement (column), at the condition that a second movement (row) can be performed. Table 4.6 shows the percentage of participants that can perform a specific movement (column) at the condition of not being able the perform the second movement (row). When participants can perform one of the more complex movements (II to V) it is more than $90 \%$ likely that they can also perform a rolling (I) movement. Participants that can perform the cloverleaf (II) are the best all-round by being able to perform on average more than $60 \%$ of all other movements. Also interesting is that $75 \%$ of participants that can twist to the left, can also twist their tongue to the right and vice versa. Not possessing the ability to perform a certain movement never excludes the ability to perform one of the others.

Table 4.5 - Conditional probability of being able to perform a certain movement, but also another movement: If $\operatorname{Row}(\mathrm{R})$, Than Column(C)

\begin{tabular}{llllll}
\hline $\boldsymbol{R} \rightarrow \boldsymbol{C}$ & I: Rolling & II: Cloverleaf & III: Folding & $\begin{array}{l}\text { IV: Twisting } \\
\text { (left) }\end{array}$ & $\begin{array}{l}\text { V: Twisting } \\
\text { (Right) }\end{array}$ \\
\hline I: Rolling & $100 \%$ & $20 \%$ & $33 \%$ & $43 \%$ & $42 \%$ \\
\hline II: Cloverleaf & $97 \%$ & $100 \%$ & $62 \%$ & $66 \%$ & $65 \%$ \\
\hline III: Folding & $94 \%$ & $36 \%$ & $100 \%$ & $62 \%$ & $61 \%$ \\
\hline IV: Twisting (left) & $95 \%$ & $30 \%$ & $49 \%$ & $100 \%$ & $75 \%$ \\
\hline V: Twisting (right) & $95 \%$ & $30 \%$ & $49 \%$ & $76 \%$ & $100 \%$ \\
\hline
\end{tabular}

Table 4.6 - Conditional probability of not being able to perform a certain movement, but being able to perform another movement: If not Row (R), Than Column (C)

\begin{tabular}{llllll}
\hline$\sim \boldsymbol{R} \rightarrow \boldsymbol{C}$ & I: Rolling & II: Cloverleaf & III: Folding & $\begin{array}{l}\text { IV: Twisting } \\
\text { (left) }\end{array}$ & $\begin{array}{l}\text { V: Twisting } \\
\text { (Right) }\end{array}$ \\
\hline I: Rolling & $0 \%$ & $3 \%$ & $11 \%$ & $13 \%$ & $13 \%$ \\
\hline II: Cloverleaf & $82 \%$ & $0 \%$ & $23 \%$ & $32 \%$ & $32 \%$ \\
\hline III: Folding & $80 \%$ & $10 \%$ & $0 \%$ & $28 \%$ & $28 \%$ \\
\hline IV: Twisting (left) & $78 \%$ & $10 \%$ & $18 \%$ & $0 \%$ & $15 \%$ \\
\hline V: Twisting (right) & $78 \%$ & $10 \%$ & $19 \%$ & $15 \%$ & $0 \%$ \\
\hline
\end{tabular}

\subsection{DISCUSSION}

To our knowledge, we are the first to study the distribution of distinctive tongue movements and the awareness thereof in a large population. Rolling the tongue was the movement that could be performed by most people (83.7\%). The cloverleaf seemed to be the hardest movement and could only be performed by 13.7\%. The correct judgment of the ability to perform a tongue movement ranges between $\mathbf{6 3 . 1 \%}$ and $\mathbf{9 0 . 1 \%}$. A relationship between gender or right-handedness 
[15] and the ability to perform certain tongue movements, as in other studies, could not be found $[8,10-14,16]$. There were however slightly more women that could perform a total of 3 or 4 movements.

\section{Literature comparison}

According to other literature, $60 \%$ to $80 \%$ of the population can roll their tongue [8-15]. In our study, this number is even higher; $83.7 \%$ (Table 4.3a). The percentage of people being able to fold (III) their tongue is much higher in our sample $(27.5 \%)$ than in previous research (1.5\% to $3 \%)[9,10,17]$. While the folding movement can be difficult to determine, as there are different levels of folding which could potentially be judged differently, it is still an exceptionally high difference. This might indicate regional differences for this type of movement as the aforementioned research was conducted in China, India, and the United States $[9,10,17]$. Whether tongue training affects mobility is not clear. Hirschhorn (1970)[24] investigated different tongue features in a single family and found that two family members of a study population of thirteen, were able to learn tongue folding within two weeks of training. The evidence, however, is low because of the small study population. Kothari et al. [25] showed that one hour of tongue training results in an improvement of tongue strength, which is not influenced by the ability of tongue rolling.

Since others found that tongue folding might also depend on practice, it could as well be related to the language $[24,25]$. There is, however, not enough material to confirm such a theory. Being able to twist the tongue to one side doesn't necessarily imply that someone can twist to the contralateral side since only $75 \%$ of the people who can twist the tongue can do this bilaterally (Table 4.5).

The ability to roll the tongue is believed to be caused by a dominant gene, whereas the ability to fold the tongue is believed to be of a recessive character [11,16,17]. Some reports state that the ability to fold the tongue depends on the rolling tongue gene $[9,10,18]$. In this study, these results are not observed, as $11 \%$ of the people that can fold the tongue cannot roll their tongue (Table 4.6). This is strengthened by studies among monozygotic and dizygotic twins that suggest that tongue rolling is not entirely genetically determined [13,26,27]. Around $95 \%$ of people that can perform a complex movement other than rolling will also be able to roll their tongue. It seems that this movement is often a prerequisite to perform other movements.

While the shape and size of the tongue differ greatly between persons, the muscular arrangement in humans seems to follow astrict pattern $[19,20]$. However when looking at motor innervation, a different picture arises. The hypoglossal nerve has two different types of possible topologies: single branching (40\%) and 
multiple branching (60\%) [19]. However, previous research from our group shows that a specific global branching topology is not limited to characteristic muscle activity. Even within one patient, both sides of the tongue can have different distal branching topologies resulting in different muscle activations on a micro level [28]. The variety in distal branching patterns might be the reason that there are so many differences between the tongue abilities of people. Also, this study showed that of the subjects who can perform a tongue twist, not everybody can perform a twist to both the left and the right side, confirming that there is a difference in lateral innervation (Table 4.5). This could as well be one of the core concepts explaining why patients with the same tumor characteristics evolve different speech and swallowing problems [4].

\section{Judgment regarding own abilities}

Whether there is a relationship between the ability to perform complex tongue movements and postoperative tongue function remains to be investigated in future research. However, we do know that when a tumor restricts the mobility of the tongue, specific tongue abilities might be lost. Therefore, when conducting a study into this matter it is essential to know if people can judge their tongue mobility before demonstrating.

The majority (83.7\%) of the population was able to roll the tongue (I) and only $14.7 \%$ were able to fold the tongue (Table 4.3a). Both abilities are slightly underestimated by the population, but the "wrong judgment" is very low $(9.9 \%$ and $13.1 \%)$. The low level of wrong judgments makes these abilities potentially useful to distinguish patients in postoperative functionality. However, since the ability for rolling is relatively common and folding relatively uncommon, there is less room to differentiate between groups. The group that can roll, but not a cloverleaf is by far the largest.

About one-third of the population can perform a folding (III) movement or a twisting (left/right) movement. This $1 / 3$ ratio is more ideal for decision making since the subdivided groups have more balanced sizes. These abilities are, however, often overestimated. The wrong judgment, which ranges from $24.1 \%$ to $36.9 \%$, largely consists of people who think they can perform this movement, but cannot. With these movements, the physician can only be confident in $63.1 \%$ to $73.9 \%$ of the cases that the patient can make a proper judgment about their ability.

By looking at the percentage of wrong judgment, tongue rolling and cloverleaf seem to be the best abilities to test for a post-operative prediction model. However, discrepancies between the specific tongue movements are large. Cloverleaf performance is found in $14,7 \%$, whereas tongue rolling is present in 
$83,7 \%$. This means that there are a large number of people, who can perform rolling but cannot perform cloverleaf. These observations limit the number of patients that could benefit from the model and pose a potential problem for future study designs. Nevertheless, we are convinced that knowledge of the effect of preoperative tongue movements on postoperative mobility can be used to gain more insight into the mechanisms that influence the success rate of rehabilitation post-surgery.

\section{Limitations}

Both observers discussed the scoring of different movements beforehand. However, judging whether someone can perform a movement can be a matter of interpretation. It appeared, that mainly small differences emerge due to different interpretations by the observer despite the use of clear images. The chi-square test and manual check of the frequencies confirm that no significant incongruencies occurred. Limited data about tongue movements have been published since the first article in 1940. Neither are demonstration materials other than images available. Therefore it was decided to use the five instructive images from a previous paper on this subject [7]. Yet it is unknown if, for example, video instructions would result in different performances by the participants.

Because all participants were recruited on institute premises, it is questionable if it does represent the Dutch population in all its aspects. It would, for example, be interesting to subdivide groups in different dialects spoken in the Netherlands or based on ethnicity. Nevertheless, this topic was out of the scope of this study.

\subsection{FUTURE PERSPECTIVES}

Knowing if, and how many possess the ability to perform any of the specific tongue movement is a prerequisite for further research towards predicting the functional outcome. Future research could focus on assessing if there is a difference in functional outcome after treatment between the patients that can roll the tongue, can make a cloverleaf, both or neither of these. This potential prediction model can be used in combination with or instead of more advanced techniques such as biomechanical modeling or optical tracking. These options might take longer to implement and validate in a clinical setting [29,30]. Also when the genetics and physiology behind the ability to perform different features can be clarified, it might increase insights in the prediction of post-operative reduced tongue mobility or may be helpful in oral rehabilitation. 


\subsection{CONCLUSION}

Tongue movement features and its distribution in population have an unknown physiological background and have not received much scientific attention yet. This study assessed the distribution of five specific tongue movements in the Dutch population: rolling: $83.7 \%$, Cloverleaf: $14.7 \%$, Folding: $27.5 \%$, Twisting left: $36.1 \%$, and Twisting right: $35.6 \%$. An additional finding is that the percentage of people that can fold their tongue is almost ten times higher (3\% versus $27.5 \%$ ) than in previous research. It was found that the ability to roll the tongue is not a prerequisite for folding of the tongue.

In this study, participants were most aware of their ability to roll the tongue and to make a cloverleaf. While the group size difference between people who can and cannot do these movements is relatively large, rolling and cloverleaf are still preferred over folding and twisting (left and right) for future prediction models, based on the low number of misjudgments with these movements. 


\subsection{REFERENCES}

1. Konstantinović VS, Dimić ND. Articulatory function and tongue mobility after surgery followed by radiotherapy for tongue and floor of the mouth cancer patients. Br J Plast Surg. 1998;51: 589-593. doi:10.1054/bjps.1998.0202

2. Speksnijder CM, van der Bilt A, van der Glas HW, Koole R, Merkx MAW. Tongue function in patients treated for malignancies in tongue and/or floor of mouth; a one year prospective study. Int J Oral Maxillofac Surg. International Association of Oral and Maxillofacial Surgery; 2011;40: 1388-1394. doi:10.1016/j.ijom.2011.09.003

3. Bressmann T, Sader R, Whitehill TL, Samman N. Consonant intelligibility and tongue motility in patients with partial glossectomy. J Oral Maxillofac Surg. 2004;62: 298-303. doi:10.1016/j.joms.2003.04.017

4. Matsui Y, Ohno K, Yamashita Y, Takahashi K. Factors influencing postoperative speech function of tongue cancer patients following reconstruction with fasciocutaneous/ myocutaneous flaps-a multicenter study. Int J Oral Maxillofac Surg. 2007;36: 601609. doi:10.1016/j.ijom.2007.01.014

5. Lazarus CL, Husaini H, Jacobson AS, Mojica JK, Buchbinder D, Okay D, etal. Development of a new lingual range-of-motion assessment scale: Normative data in surgically treated oral cancer patients. Dysphagia. 2014;29: 489-499. doi:10.1007/s00455-014-9534-9

6. Kamstra JI, Jager-Wittenaar H, Dijkstra PU, Huisman PM, van Oort RP, van der Laan BFAM, et al. Oral symptoms and functional outcome related to oral and oropharyngeal cancer. Support Care Cancer. 2011;19: 1327-1333. doi:10.1007/s00520-010-0952-4

7. Lu M. Anthropologic studies of five types of tongue movements among ethnic groups in China. Commun Contemp Anthropol. 2013; 23-28. doi:10.4236/coca.2013.71003

8. Sturtevant AH. A New Inherited Character in Man. Proc Natl Acad Sci. 1940;26: 100102. doi:10.1073/pnas.26.2.100

9. Liu TT, Hsu TC. Tongue-folding and tongue-rolling: In a sample of the chinese population. J Hered. 1949; doi:10.1093/oxfordjournals.jhered.a105945

10. Gahres EE. Tongue rolling and tongue folding: And other hereditary movements of the tongue. J Hered. 1952;43: 221-225. doi:10.1093/oxfordjournals.jhered.a106313

11. Ubbanowski A, Wilson J. Tongue curling. J Hered. 1947;38: 365-366. doi:10.1093/ oxfordjournals.jhered.a105674

12. He X, Zhang JF, Li ZX, Liu C, Yang LT, Wang N, et al. The traits of five types of tongue movement in Han of Shaanxi, China. Anat Sci Int. 2012;87: 181-186. doi:10.1007/ s12565-012-0141-2

13. Lee JW. Tongue-folding and tongue-rolling: In an american negro population sample. J Hered. 1955;46: 289-291. doi:10.1093/oxfordjournals.jhered.a106581

14. Azimi-Garakani C, Beardmore JA. Tongue-rolling phenotypes and geographical variation in the United Kingdom. Anthropol Anz. 1989;47: 305-310. doi:10.2307/29540011

15. Fry CJ. Left-handedness and tongue-rolling ability. Percept Mot Skills. 1988;67: 168170. doi:10.2466/pms.1988.67.1.168

16. Hsu TC. Tongue upfolding: A newly reported heritable character in man. J Hered. 1948;39: 187-188. doi:10.1093/oxfordjournals.jhered.a105848

17. Whitney DD. Tongue tip overfolding. J Hered. 1949;40: 18. doi:10.1093/oxfordjournals. jhered.a105944 
18. Singh MR. Tongue Rolling and Folding in Six Populations of Manipur, India. Front Anthropol. 2012; 41-45.

19. Mu L, Sanders I. Human tongue neuroanatomy: Nerve supply and motor endplates. Clin Anat. 2010;23: 777-791. doi:10.1002/ca.21011

20. Sanders I, Mu L. A three-dimensional atlas of human tongue muscles. Anat Rec. 2013;296: 1102-1114. doi:10.1002/ar.22711

21. Van Der Molen L, Van Rossum MA, Burkhead LM, Smeele LE, Rasch CRN, Hilgers FJM. A randomized preventive rehabilitation trial in advanced head and neck cancer patients treated with chemoradiotherapy: Feasibility, compliance, and short-term effects. Dysphagia. 2011; doi:10.1007/s00455-010-9288-y

22. KraaijengaSAC,MolenLvander,StuiverMM,TakesRP,Al-MamganiA,BrekelMWMva.den, etal.Efficacyofanovelswallowingexerciseprogramforchronicdysphagiainlong-termhead and neck cancer survivors. Head Neck. 2017;39: 1943-1961. doi:10.1002/hed.24710

23. Statistics How To [Internet]. Available: https://www.statisticshowto.datasciencecentral. $\mathrm{com} /$

24. Hirschhorn HH. Transmission and learning of tongue gymnastic ability. Am J Phys Anthropol. 1970;32: 451-454. doi:10.1002/ajpa.1330320315

25. Kothari M, Svensson P, Basic A, Christiansen B, Vigsø M, Truc L, et al. Influence of the ability to roll the tongue and tongue-training parameters on oral motor performance and learning. Arch Oral Biol. 2011;56: 1419-1423. doi:10.1016/j.archoralbio.2011.04.017

26. Matlock P. Identical twins discordant in tongue-rolling. J Hered. 1952;43: 24. doi:10.1093/oxfordjournals.jhered.a106251

27. Reedy JJ, Szczes TS, Downs TD. Tongue rolling among twins. J Hered. 1971;62: 125127. doi:10.1093/oxfordjournals.jhered.a108139

28. Van Alphen MJAJA, Eskes M, Smeele LEE, Balm AJMJM, van der Heijden F. In vivo intraoperative hypoglossal nerve stimulation for quantitative tongue motion analysis. Comput Methods Biomech Biomed Eng Imaging Vis. Taylor \& Francis; 2017;5: 409_ 415. doi:10.1080/21681163.2015.1072056

29. Kappert KDR, van Alphen MJA, Smeele LE, Balm AJM, van der Heijden F. Quantification of tongue mobility impairment using optical tracking in patients after receiving primary surgery or chemoradiation. Kimple RJ, editor. PLoS One. 2019;14: e0221593. doi:10.1371/journal.pone.0221593

30. Kappert KDR, van Alphen MJA, van Dijk S, Smeele LE, Balm AJM, van der Heijden F. An interactive surgical simulation tool to assess the consequences of a partial glossectomy on a biomechanical model of the tongue. Comput Methods Biomech Biomed Engin. 2019;22: 827-839. doi:10.1080/10255842.2019.1599362 


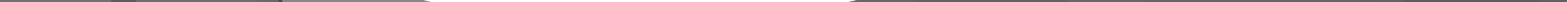




\section{IN-VIVO TONGUE STIFFNESS MEASURED BY ASPIRATION: RESTING VS GENERAL ANESTHESIA.}

K.D.R. Kappert

N. Connesson

S.A. Elahi

S. Boonstra

A.J.M. Balm

F. van der Heijden

Y. Payan

This chapter is resubmitted after minor revisions to the Journal OfBiomechanics

This work was presented at the 16th International Symposium on Computer Methods in Biomechanics and Biomedical Engineering and the 4th Conference on Imaging and Visualization, New York, 2019 


\section{ABSTRACT}

Tongue cancer treatment often results in impaired speech, swallowing, or mastication. Simulating the effect of treatments can help the patient and the treating physician to understand the effects and impact of the intervention. To simulate deformations of the tongue, identifying accurate mechanical properties of (fibrotic) tissue is essential. However, not many succeeded in characterizing invivo tongue stiffness. Those who did, measured the tongue At Rest (AR), in which muscle tone subsides even if muscles are not willingly activated. We expected to find an absolute rest state in participants 'under General Anesthesia' (GA).

We elaborated on previous work by measuring the mechanical behavior of the in-vivo tongue under aspiration using an improved volume-based method. Using this technique, 5 to 7 measurements were performed on 10 participants both AR and under GA. The obtained Pressure-Shape curves were first analyzed using the initial slope and its variations. Hereafter, an inverse Finite Element Analysis (FEA) was applied to identify the mechanical parameters using the Yeoh, Gent, and Ogden hyperelastic models.

The measurements AR provided a mean Young's Modulus of $1638 \mathrm{~Pa}$ (min 1035 max 2019) using the Yeoh constitutive model, which is in line with previous ex-vivo measurements. However, while hoping to find a rest state under GA, the tongue unexpectedly appeared to be approximately 2 to 2.5 times stiffer under GA than AR. Explanations for this were sought by examining in drugs administered during GA, blood flow, perfusion, and upper airway reflexes, but neither of these explanations could be confirmed. 


\subsection{INTRODUCTION}

Head and neck cancers are notorious for their negative impact on quality of life [1-6]. Treatment of tongue cancer, which accounts for approximately $20 \%$ of all head and neck carcinomas, can have a destructive effect on speech, swallowing, and mastication. The preferred treatment is surgery, but if the resulting postoperative functional loss is expected to be too disabling, organ-preserving chemoradiation and other alternatives can be considered $[7,8]$. Due to the complex structures and systems involved in oral functions, a clinician cannot accurately predict the functional consequences of treatment. Effective patient counseling and treatment choice is, therefore, often an arduous task. Simulating the effect of treatments using biomechanical computer models can help the patient and the treating physician to understand the impact of interventions. In the past years, multiple biomechanical tongue models have been developed to simulate tongue function with and without simulated impairment caused by pathologies and/or treatment [9-13].

To simulate realistic tissue behavior, choosing a proper constitutive model and identifying accurate mechanical properties is essential. Surgery or radiation can also induce fibrotic changes to tissue from which the extent may vary between patients and will affect the mechanical properties. Moreover, even for basic parameters such as the in-vivo stiffness values of the tongue in rest, no consensus has yet been reached [14].

The human tongue mainly consists of muscles that behave as a nonlinear, timedependent, inhomogeneous, and anisotropic material. Most of the constitutive model parameters in literature applied in human tongue tissue simulations are not based on real data because of the associated experimental difficulties [1520]. According to Hermant et al. [14], only three papers used experimental data to estimate the average tongue stiffness. Two of these experiments were performed in-vivo [21,22] and one ex-vivo [15]. In-vivo magnetic resonance elastography (MRE) was used by in seven healthy participants, for which they found a mean Young's modulus of $7743 \mathrm{~Pa}$. The MRE measurements have two main downsides. First, due to the supine position of the individual, the tongue muscles are activated to keep an acceptable geometry, thus providing higher stiffness values compared to what is expected for a tongue AR. Second, in the setup proposed by Cheng et al. (21), the mechanical properties are retrieved by analyzing the displacement field induced by external $80 \mathrm{~Hz}$ vibrations transferred into the tongue tissue [21]. Thus, the measured stiffness modulus may differ from the quasi-static elastic modulus due to the expected muscle frequency-dependent mechanical behavior [23]. 
Schiavone et al. [22] measured tongue properties in-vivo while the participant willingly activated or relaxed their tongue muscles using an aspiration method. In this method, a chamber with a circular aperture was placed on the tongue and depressurized. The height of the tissue aspirated into the chamber was measured using a camera. Inverse identification of the tissue parameters was then performed using a Finite Element (FE) model of the aspiration experiment and Yeoh strain energy function.

Ex-vivo tissue is expected to have a higher Young's modulus because of the lack of vascularization and protein degradation $[24,25]$. However, the ex-vivo experiment of Gerard et al. [15] (Table 5.5) showed a tongue Young's Modulus that was almost 2 times lower than the in-vivo-experiment of Schiavone et al. [22] AR. With the same measurement system but activated muscles, the observed Young's Modulus was approximately 6 times higher [22]; muscle tongue activation thus impacts the observed stiffness. Furthermore, it should be noted that residual stresses and natural muscle tone persist even if muscles are not willingly activated [26].

These phenomena raise the difficulty to define a reference muscle state, to perform an in-vivo measurement, and to set a simulation start point. From a practical point of view, this reference muscle state will always be a situation where internal stress and strain heterogeneities are present. It is the authors' opinion that such a reference muscle state should be defined with the lowest possible muscle activity, i.e. when only muscle tone remains. We expect to find this 'absolute' rest state in participants under General Anesthesia (GA).

Using an improved version of the aspiration method, initially presented in Elahi et al. [27,28], the tongue mechanical properties will be measured (1) 'at rest' (AR) and (2) under GA, on 10 individuals without any history of tongue disease. As a first approximation, the tongue tissue will be considered as a homogeneous uniform isotropic material. The used inverse identification method and the impact of different constitutive models will be discussed. The main goals of this study are to estimate the following for the human tongue:

- The measurement reproducibility in each participant and variability between participants.

- The differences in apparent behavior of tissue between AR and under GA.

- The tissue parameters of the tongue for simulation purposes. 


\subsection{METHOD}

\section{Measurement setup}

Elahi et al. [27] proposed a modified version of the aspiration device of Schiavone et al. [22] (Figure 5.1A). Any camera was removed from the system to diminish design constraints; the measurement of the tissue height was replaced by a volume measurement using a medical pump. It provided results with a maximum error of $+8.8 \%$ compared to classical tests such as tensile or bulge tests. The system and method used in this paper are comparable to the one used in Elahi et al. [28] where more details are reported.

In this work, a custom suction cup (Figure 5.1B and C) was printed in 3D with a Form 2 printer (Formlabs, Somerville USA) using dental SG (biocompatible resin). The suction cup has an inner diameter of $10 \mathrm{~mm}$ and a wall thickness of $0.5 \mathrm{~mm}$. (Figure 5.1B).

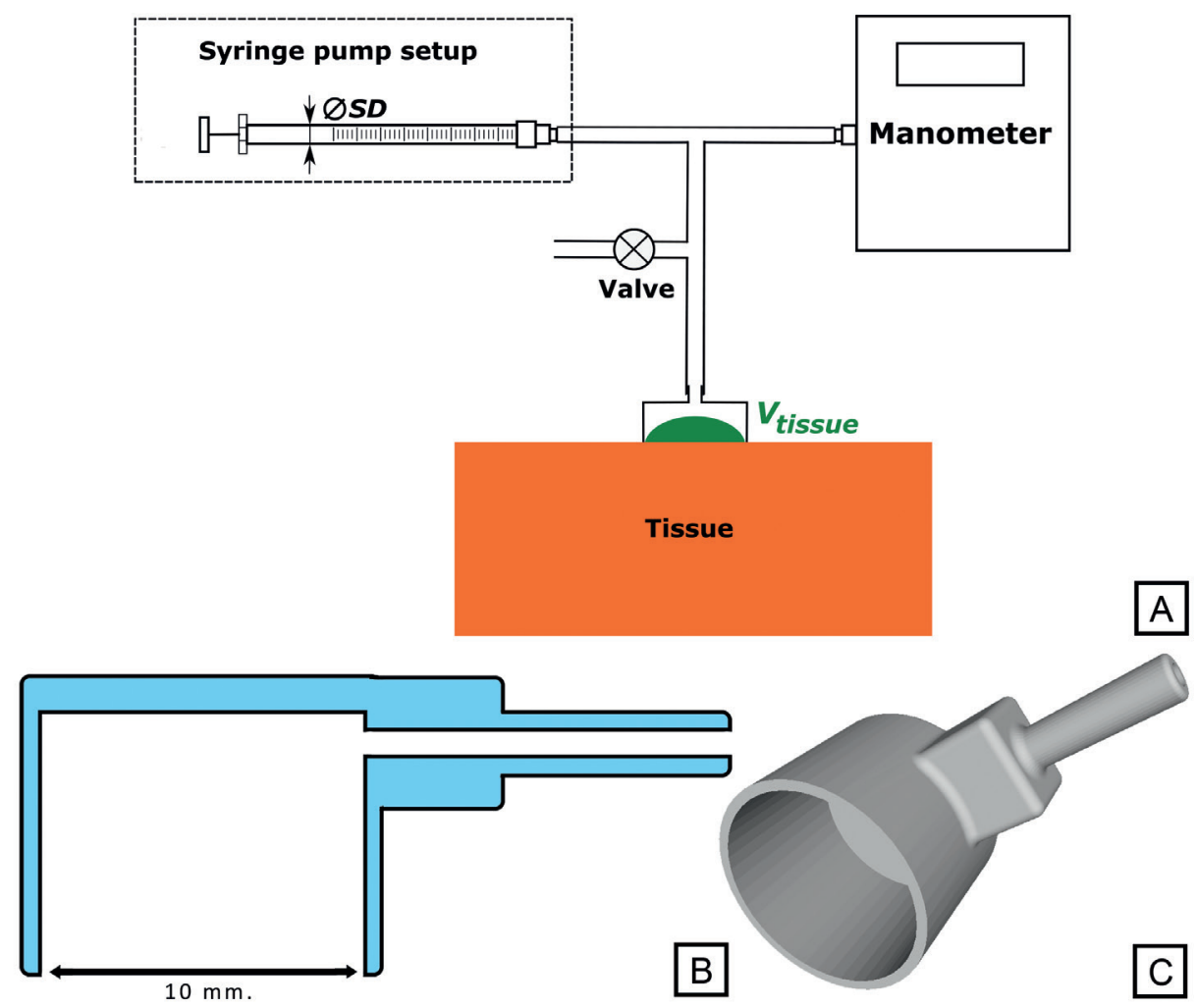

Figure 5.1 - A schematic drawing of the volume-based aspiration setup (A) Adapted from Elahi et al. [28], the suction cup cross-section with an aperture diameter of $10 \mathrm{~mm}$, a wall thickness of $0.5 \mathrm{~mm}$, and side tube (B), and a 3D render of the cup (C). 
A programmable syringe pump (pump 11 elite, Harvard Apparatus, Holliston USA) was used to remove an air volume $V^{\text {total }}$ from the system at a fixed rate of $0.6 \mathrm{~mL} \cdot \mathrm{min}^{-1}$. The syringe pump in combination with a $3 \mathrm{~mL}$ syringe provided a precision of $\pm 0.002 \mathrm{ml}$ [28]. The corresponding pressure $P$ was measured using a manometer (DP205, MECOTEC GmbH, Hattingen, Germany) with a precision of around 0.004 mbar. The measured total volume curve $V^{\text {total }}(P)$ retrieved from the system consisted of both the tissue aspirated inside the suction cup and changes in the intrinsic system volume (air expansion and stiffness of components). The changes in system volume $V_{\text {system }}$ were assessed by testing a non-deformable material (internal calibration) whereby only the response of the system $V_{\text {system }}(P)$ is measured. The aspirated tissue volume at each pressure was then estimated using the following relation:

$$
V_{\text {tissue }}(P)=V^{\text {total }}(P)-V_{\text {system }}(P)
$$

The tissue volume $V_{\text {system }}(P)$ was normalized into an adimensional and scaleindependent Shape parameter $S$ using a reference volume of the tissue bump inside the suction cup, which has the shape half a sphere (Figure 5.1A). A typical Pressure-Shape obtained on human tongue tissue is plotted in Figure 5.2. Such a curve contains information about the mechanical properties of the tongue tissue.
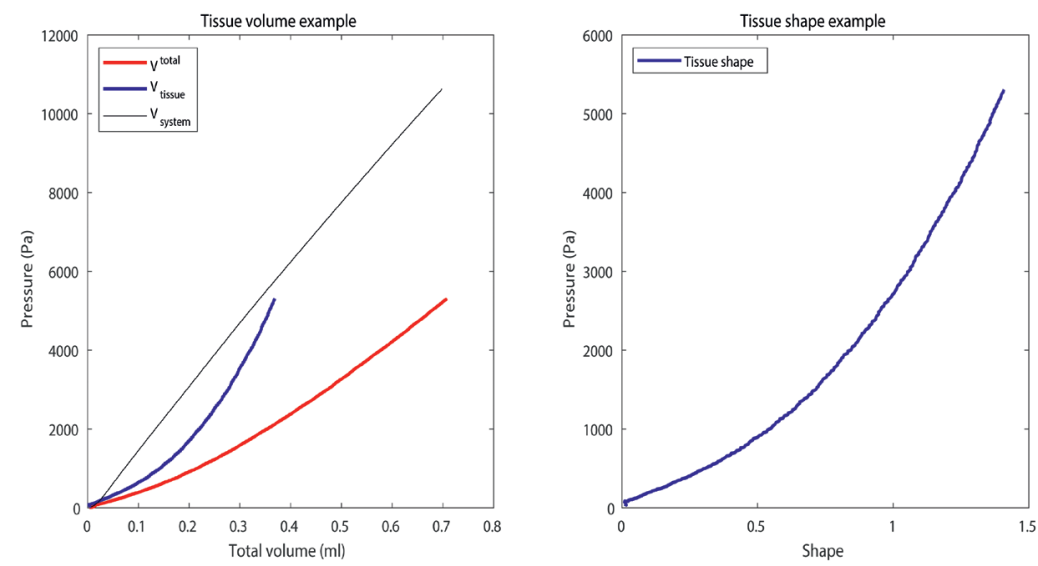

Figure 5.2 - The left graph shows the raw experimental data and associated tissue volume $V_{\text {tissue }}(P)$ and the right graph shows the normalized curve.

\section{Measurement protocol}

The measurements AR and under GA were performed, where possible, using identical protocols. The aim was to include ten participants scheduled for surgery without any history of tongue disease. Therefore, patients at the gynecology and urology department of the Netherlands Cancer Institute were approached to participate. Since this was the first study of its kind, sample size calculation was 
not applicable. The measurements AR were performed on seated participants a week before or a month after surgery. Measurements under GA started one hour after the beginning of surgery. All participants had an epidural catheter and were infused according to the same anesthesiologic protocol with solutions of Propofol, Sufentanil, Remifentanil, Efidrine, and Rucurionium using different dosages.

A sterilized suction cup was placed on the dorsum of the tongue, away from the midline (Figure 5.3).A minimum of five measurements was performed successively, each time removing the cup for 30 seconds between each measurement so that the tissue had time to restore to its initial state. When possible, the measurement location was alternated (Figure 5.3A, yellow areas) to avoid waiting time.
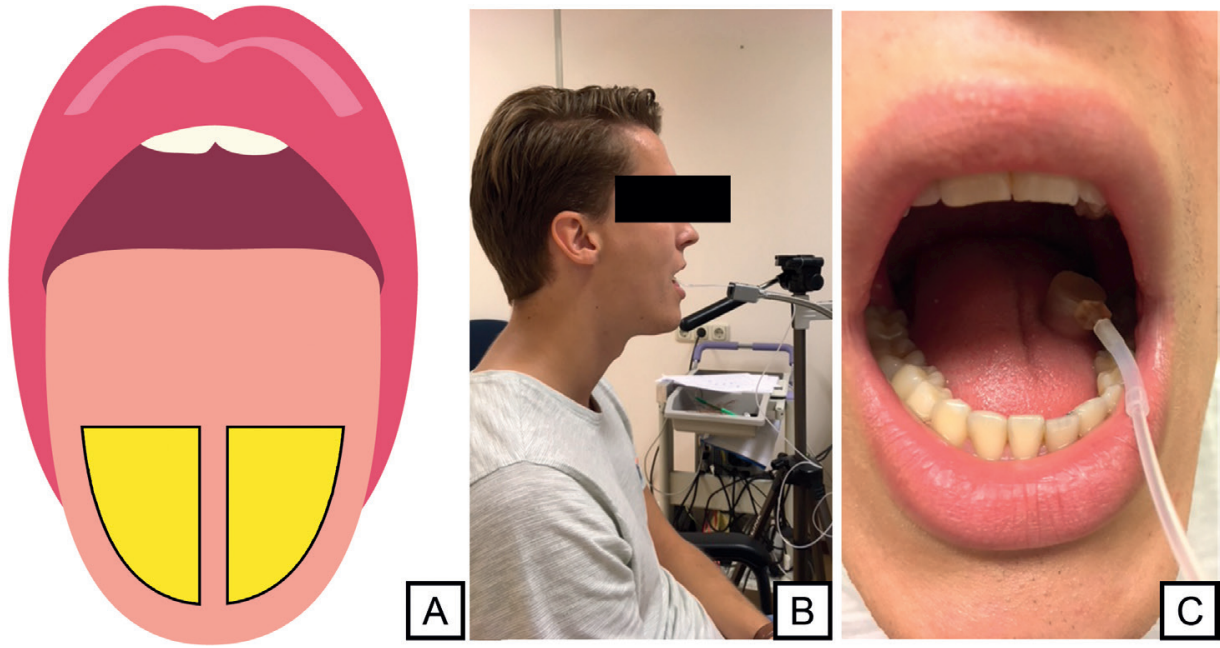

Figure 5.3 - An Illustration of the human tongue (A) and the applied suction cup on the tongue of a participant (B, C). During the measurements, a sterilized cup is placed on the yellow area on the tongue's surface. (Background image designed by Freepik)

For each measurement, the pressure was monitored before placing the suction cup. A small initial pre-load, applied by placing the cup by hand, helped to prevent leakage. The pressure change due to this pre-load was monitored and subsequently removed from the experimental data. Any initial leakage could be detected during this stage; erroneous measurements were immediately discarded.

The syringe pump was started using custom software to synchronize pump start and pressure measurement. During the development of the device, discomfort was reported at a pressure lower than approximately -80 mbar. Therefore, all measurements in this study were thus stopped at a pressure threshold of -55 mbar to prevent any discomfort. 
All procedures performed in studies involving human participants were in accordance with the ethical standards of the medical ethical committee of the Netherlands Cancer Institute (ref: N18EMT) and with the 1964 Helsinki declaration and its later amendments or comparable ethical standards. Informed consent was obtained from all individual participants included in the study.

\section{Tissue property analysis and parameter identification}

The main features of the experimental Pressure-Shape curves $S_{\text {exp }}(P)$ (Figure 5.2) are its initial slope and variation with pressure level. These two features are impacted by the apparent behavior of the material (Stiffness, Incompressibility, strain-stiffening relation) but also by additional phenomena such as the residual load applied to the cup, the cup-tissue friction coefficient, the tongue size compared to aperture diameter, the boundary conditions, the experimental reproducibly difficulties due to system temperature change, the cup positioning. The extracted quantitative values of material parameters are impacted by the hypotheses of these parameter ranges, but also by the very choice of the constitutive model.

To circumvent these difficulties during the first steps in the analysis, the PressureShape experimental curves results were analyzed by evaluating the curve slope $A_{R S}$ at a Reference Shape $R_{S}=0.1$ :

$$
A_{R S}=\left.\frac{d P}{d S_{\text {exp }}}\right|_{S_{\text {exp }}=R_{S}}
$$

The Pressure-Shape stiffening was characterized by the ratio $B_{\text {stiff }}$ dividing the curve slope $A_{R S}$ at $R_{S}=0.5$ by $R_{S}=0.1$ :

$$
B_{\text {Stiff }}=\frac{A_{0.5}}{A_{0.1}}
$$

In this study, stiffness $A_{R S}$ and stiffening ratio $B_{\text {Stiff }}$ were compared for both AR and under GA.

In a second step, the quantitative range of the Young's Modulus was extracted from the experimental data. For this, an inverse analysis was performed by simulating the measurement Pressure-Shape $S_{F E}(P)$ using a Finite Element (FE) model as also used by Elahi et al. [27] (Figure 5.4a). The test was modeled in $2 \mathrm{D}$ assuming an axisymmetric axis in a FE software (ANSYS). The aperture of the cup was clamped and a friction coefficient of $\mu=0.2$ was chosen for the contact between the tissue and the cup. Negative pressure was applied on the surface inside the cup and the outer tissue boundaries remained free. This method was successfully validated on a silicone phantom [28]. 


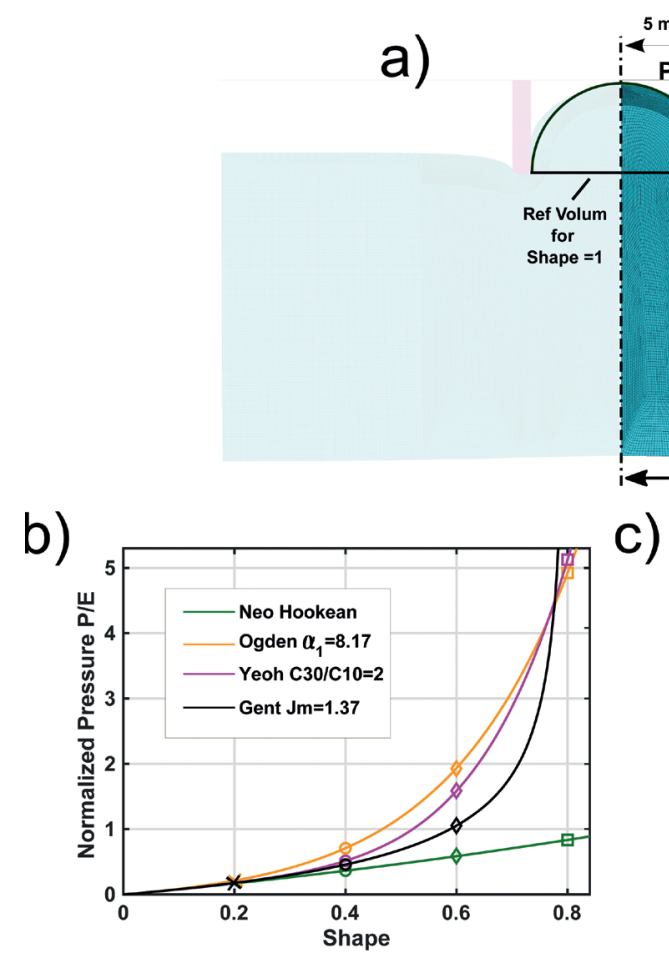

c)

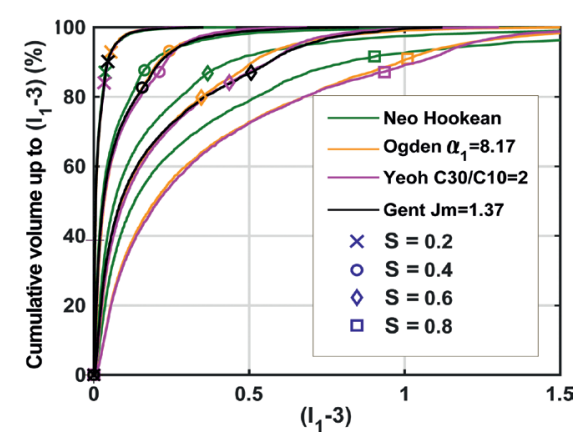

Figure 5.4 - a) Simulated FE, Boundary conditions, meshing (36000 Q8 axisymmetric elements), and an example of the deformed shape $S=1$. b) Normalized Pressure-Shape curve for different material models and chosen parameters representative of the experimental results. c) Illustration of cumulated volumic repartition in the tissue under the aperture (Depth=Radius=0.75 Diameter) for which local invariant is lower than an $\left(\mathrm{I}_{1}-3\right)$ threshold. Specific points presented in figure b) for shapes 0.2, 0.4, 0.6 and 0.8.

In this work, the minimized function was defined in using the least mean square method to optimize both shape and slope fitting error:

$$
\begin{aligned}
\emptyset & =\beta \emptyset_{1}+(1-\beta) \emptyset_{2} \\
& =\beta \underbrace{\sum_{i=1}^{I} \epsilon_{i}^{2}}_{\text {Shape fitting quality }}+(1-\beta) \underbrace{\sum_{k=1}^{K}\left(\frac{d \epsilon}{d S}\right)_{k}^{2}}_{\text {Slope fitting quality }}
\end{aligned}
$$

where

- $\epsilon_{i}$ is theshapefittingerrorforameasurementatpressure $P_{i}\left(\epsilon_{i}=\left(S_{\text {exp }}+V_{o}-S_{F E}\right)_{i}\right)$ and $V_{o}$ is the value for an unknown volume due to the plastic-rubber backlash of the syringe piston occurring at the measurement start.

$-\left(\frac{d \epsilon}{d s}\right)_{k}$ is the derivative of the fitting error $\epsilon$ regarding the shape, and is evaluated at the pressure $P_{k}$. Function part $\emptyset_{2}$ thus represents the slope fitting error at the start of the curve. 
- $I$ is the number of experimental points, $K$ is a chosen number of points so that $=\operatorname{round}(/ 5)$ to reduce the slope fitting error only at the start of the curve.

$-\beta$ is a value chosen in the range [0 1$]$ to focus the minimization process on either the shape fitting error $\epsilon_{i}$ or on its derivative at measurement start.

Different model formulations (Table 5.1, Gent [29], Yeoh [30], and Ogden [31]) have been used in their fully incompressible formulation (Poisson ratio $n=0.5$, ANSYS feature) to describe the tongue tissue behavior under aspiration.

Criteria must be defined to compare the models as they express different physical and stiffening behavior from the starting point of the Neo-Hookean model (Ogden, 1984). The model outputs have been compared studying (1) an equivalent Young's modulus (Table 1), defined using the constitutive model parameters, (2) the Pressure-Shape curve fitting quality to the experimental curves $\left(\varnothing, \varnothing_{1}, \emptyset_{2}\right.$, Eq 5.4), and (3) invariant strain fields $\left(I_{1}-3\right)$ computed using $I_{1}$, the first invariant of the Cauchy-Green strain tensor.

In this work, the strain invariant $\left(I_{1}-3\right)$ fields, for each model and for specific shapes values, have been converted into cumulated volume repartition curves $f\left(I_{1}-3\right)$ (Figure 5.4c):

$$
\mathrm{f}\left(\mathrm{I}_{1}-3\right)=\frac{\mathrm{V}_{X<\left(\mathrm{I}_{1}-3\right)}}{\mathrm{V}_{\text {ref }}}
$$

where $\mathrm{V}_{X<\left(\mathrm{I}_{1}-3\right)}$ is the tissue volume for which the value is lower than the specific threshold $\left(\mathrm{I}_{1}-3\right)$. $\mathrm{V}_{\text {ref }}$ is the considered tissue volume around the aperture. All volumes have been evaluated in the un-deformed initial state. 
Table 5.1 - Overview of different constitutive models on biological tissue and their strain energy density functions found in the literature. The equivalent Young's modulus assumes a Poisson coefficient of 0.5 .

\begin{tabular}{|c|c|c|c|c|}
\hline Model & Strain energy density function & & $\begin{array}{l}\text { Equivalent } \\
\text { Young's } \\
\text { modulus }\end{array}$ & $\begin{array}{l}\text { Application } \\
\text { illustration }\end{array}$ \\
\hline Gent & $W=-\frac{\mu J m}{2} \ln \left(1-\frac{I_{1}-3}{J m}\right)$ & $\begin{array}{l}\text { with } \mu \text { the shear } \\
\text { modulus. } J_{m} \text { is the } \\
\text { stiffening parameter } \\
\text { and its' maximum } \\
\text { value is of }\left(I_{1}-3\right) \text {. } \\
I_{1}=\lambda_{1}^{2}+\lambda_{2}^{2}+\lambda_{3}^{2}, \\
\text { where }\left(\lambda_{i}\right)_{i=1,3} \text { are } \\
\text { the principal stretches }\end{array}$ & $E=3 \mu$ & $\begin{array}{l}\text { (Elahi et al. } \\
{[27,28] \text {; }} \\
\text { Rashid et al. } \\
{[32] \text { ) }}\end{array}$ \\
\hline Ogden & $W=\frac{\mu_{1}}{\alpha_{1}}\left(\lambda_{1}^{\alpha_{1}}+\lambda_{2}^{\alpha_{1}}+\lambda_{3}^{\alpha_{1}}-3\right)$ & $\begin{array}{l}\text { where }\left(\lambda_{i}\right)_{i=1,3} \\
\text { are the principal } \\
\text { stretches, and } \\
\mu_{1} \alpha_{1}>0 . \text { With } \\
\alpha_{1} \text { the stiffening } \\
\text { parameter. }\end{array}$ & $E=\frac{3 \alpha_{1} \mu_{1}}{2}$ & $\begin{array}{l}\text { (Budday et al. } \\
\text { [33]; } \\
\text { Rashid et al. } \\
\text { [32]) }\end{array}$ \\
\hline Yeoh $_{\mathrm{c} 20 \mathrm{c} 30}$ & $\begin{aligned} W=C_{10}\left(I_{1}-3\right)+ & C_{20}\left(I_{1}-3\right)^{2} \\
& +C_{30}\left(I_{1}-3\right)^{3}\end{aligned}$ & \multirow{3}{*}{$\begin{array}{l}\text { with } I_{1} \text { the first } \\
\text { variant of the } \\
\text { Cauchy-Green strain } \\
\text { tensor. } C_{20} \text { and } C_{30} \\
\text { are the stiffening } \\
\text { parameters. }\end{array}$} & \multirow[t]{3}{*}{$E=6 C_{10}$} & \multirow{3}{*}{$\begin{array}{l}\text { (Buchaillard et } \\
\text { al. [16]; } \\
\text { Gerard et al. } \\
\text { [15]; } \\
\text { Mehrabian } \\
\text { and Samani, } \\
\text { [34]; } \\
\text { Sadeghnejad } \\
\text { et al. [35]; } \\
\text { Schiavone et } \\
\text { al. [22,36,37]) }\end{array}$} \\
\hline Yeoh $_{\mathrm{c} 20}$ & $W=C_{10}\left(I_{1}-3\right)+C_{20}\left(I_{1}-3\right)^{2}$ & & & \\
\hline Yeoh $_{\text {c30 }}$ & $W=C_{10}\left(I_{1}-3\right)+C_{30}\left(I_{1}-3\right)^{3}$ & & & \\
\hline
\end{tabular}

Additional adjustments compared to Elahi et al. [28] have been made to make this method practical for in-vivo human tongue tissue characterization. Only two adjustments will be discussed briefly as these are not the main scope of this paper:

Initial partial vacuum: contrary to Elahi et al. [28] no secondary syringe has been used to create the initial partial vacuum to prevent leakage before the start of the measurement. This step would affect the system calibration curve by modifying the active air quantity in the system tubes. In the current setup, leakage was prevented by keeping the tongue moisturized (water and saliva) and applying a 'small' initial pre-load on the suction cup. As moisturization could alter the friction coefficient, the effect hereof will be numerically assessed.

Real-time identification: while minimizing the function $\emptyset$, the measured PressureShape $S_{F E}(P)$ curves were estimated using a pre-calculated database for each 
material formulation. A model reduction based on the Principal Component Analysis method has been implemented [38]. A typical identification result is presented in Figure 5.5 using the data of patient 4 (AR).
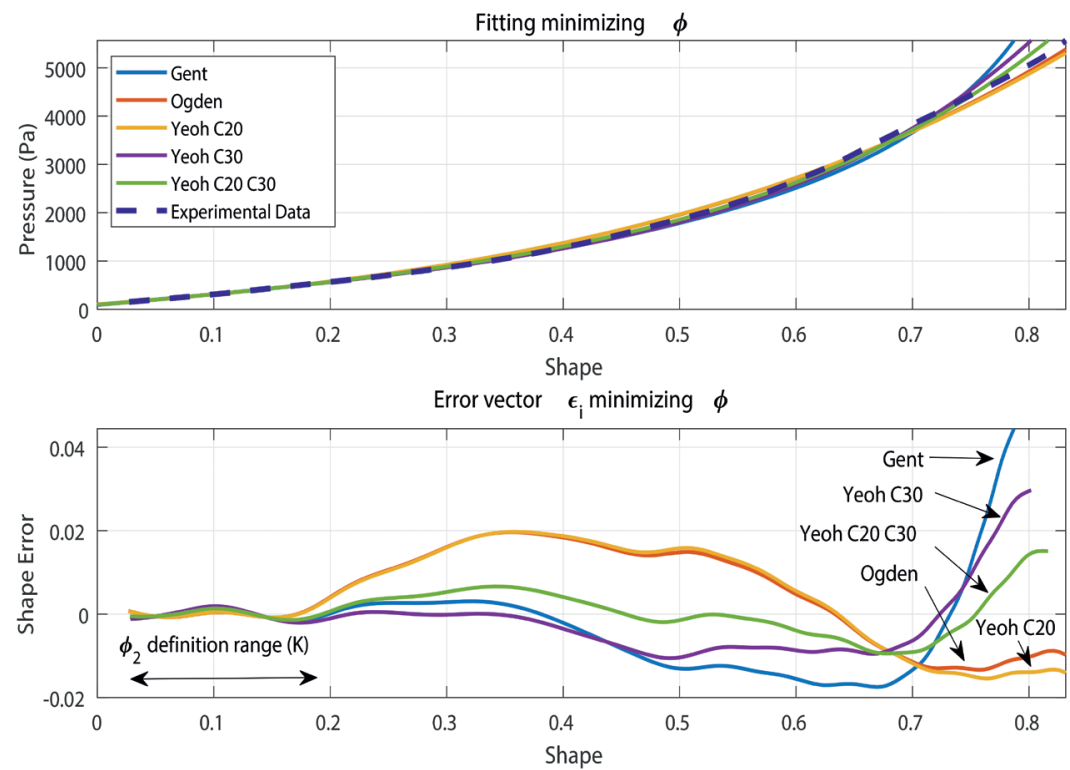

Figure 5.5 - Fitting illustration (upper graph) and associated Shape error $\epsilon_{i}$ (lower graph) of the different models minimizing $\emptyset_{2}$ in a measurement of participant \#5.

\section{Measurement variabilities}

Additional measurements were performed to evaluate ranges of reproducibility or potential biases in the measurements:

Bovine meat measurements: Seven measurements were performed ex-vivo on a piece of fresh bovine muscle at room temperature $\left(\sim 21^{\circ}\right)$ using the same protocol as the measurements on participants. Such measurement will be considered as the gold standard situation for tissue passivity and environment reproducibility.

Temperature variations: Calibration curves and measurements were measured under the same conditions at room temperature. However, while AR, exhaled air can induce temperature changes in the cup and tube extremity during the test, which is not present under GA due to the mechanical ventilator. Temperature changes impact the pressure inside the closed system during the measurement. The average effect of temperature variations was experimentally evaluated by immersing the suction cup in water with different controlled temperatures. 
Friction and Poisson coefficients: The initial Pressure-Shape slope and associated Young's Modulus identification are affected by the unknown friction and Poisson coefficient. Simulations using different friction and Poisson coefficients were performed to numerically assess the influence of these parameters.

\section{Statistical analysis:}

For the following, non-parametrical tests are used as they do not assume a Gaussian distribution. For every 5 to 7 measurements per patient, the median and the Inter Quartile Range (IQR) are calculated.

To test if the measurements AR and under GA for patient $\boldsymbol{n}$ are from the same distribution (identical median and spread) the Mann-Whitney $U$ test is used [39]. When $\mathrm{p}<0.05$, this hypothesis is rejected.

To test if the calculated medians AR and under GA over the whole population are from the same distribution, the Wilcoxon signed-rank test is used [40]. When $p<$ 0.05 , this hypothesis is rejected. The difference between the groups is accepted as significant.

The IQR is normalized by the median value to be converted in percentage to compare the variability between participants and situations:

$$
\text { Normalized IQR }=\frac{I Q R}{\text { median }} * 100 \%
$$

\subsection{RESULTS}

The characteristics of the ten participants that were included from the gynecology and urology departments at the Netherlands Cancer Institute are shown in Table 5.2. Half of the participants were males and the mean age was 62 . 
Table 5.2 - patient characteristics and number of included measurements per patient and per situation.

\begin{tabular}{llllll}
\hline $\mathbf{n}$ & Gender & Age & Type of surgery & Department & $\begin{array}{l}\text { Number of } \\
\text { measurements } \\
\text { AR/under GA }\end{array}$ \\
\hline 1 & M & 67 & Pelvic Lymph Node Dissection & URO & $5 / 6$ \\
\hline 2 & W & 62 & Bladder resection & URO & $5 / 7$ \\
\hline 3 & W & 63 & Secondary Debulking Surgery & GYN & $7 / 6$ \\
\hline 4 & M & 67 & Nephrectomy & URO & $5 / 6$ \\
\hline 5 & W & 59 & Nephrectomy & URO & $5 / 6$ \\
\hline 6 & M & 70 & Pelvic Lymph Node Dissection & URO & $6 / 6$ \\
\hline 7 & W & 49 & Secondary Debulking Surgery & GYN & $5 / 6$ \\
\hline $\mathbf{8}$ & W & 70 & Pelvic Lymph Node Dissection & URO & $6 / 7$ \\
\hline 10 & M & 55 & Ureteral Surgery & URO & $5 / 7$ \\
\hline
\end{tabular}

The median and IQR of $\mathrm{A}_{0.1}$ and $\mathrm{B}_{\text {stiff }}$ were calculated and plotted as boxplots in Figure 5.6 and Figure 5.7. The $p$ values of the Mann-Whitney $U$ test are visible on the horizontal axis. Subsequently, the means of the Medians $(\mu M)$ and the means of the IQR's $(\mu \mathrm{IQR})$ for every patient AR and under GA were calculated and are shown in Table 5.3, Figure 5.6 and Figure 5.7 for the $A_{R S}$ and $B_{\text {Stiff }}$, respectively. The table also includes the results of the ex-vivo bovine meat measurement and the results of the Wilcoxon signed-rank test. 


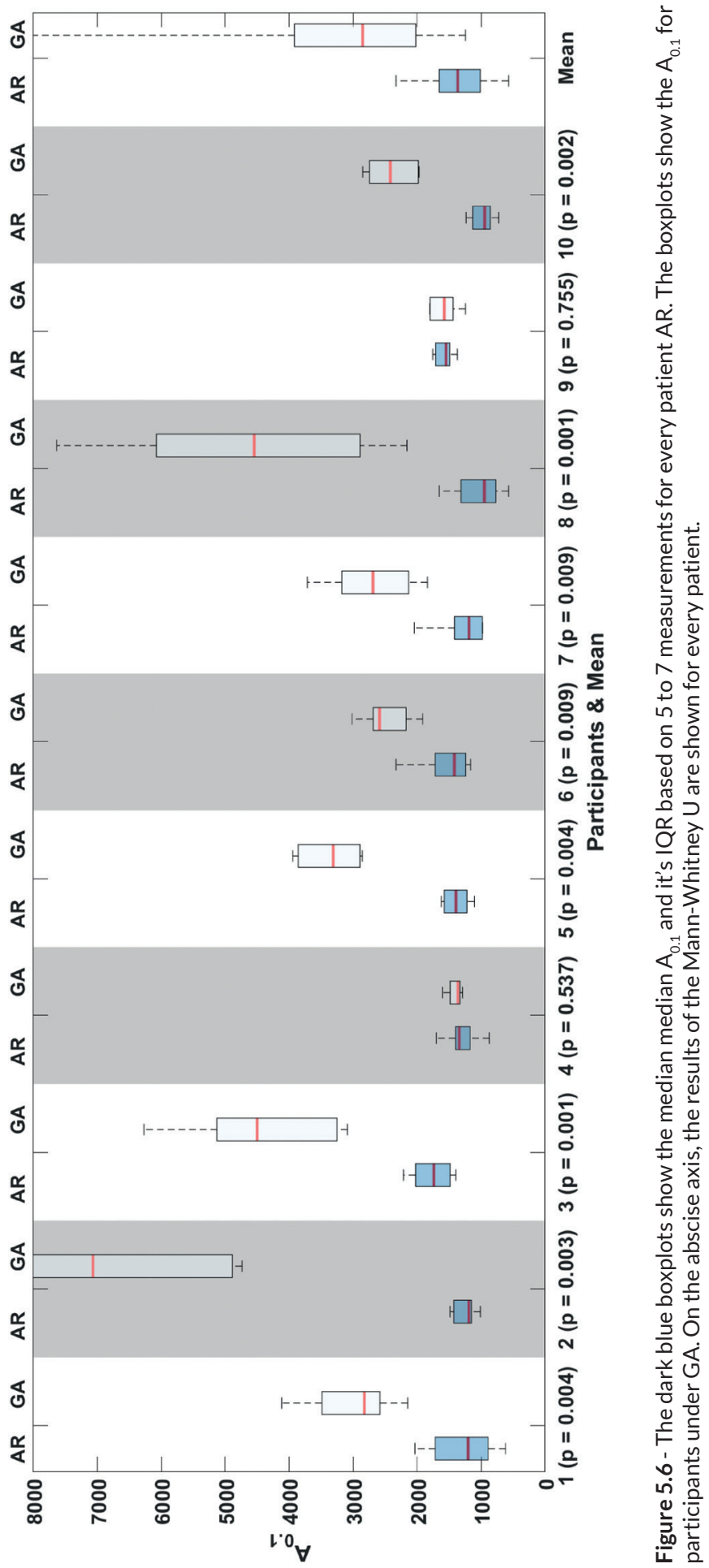




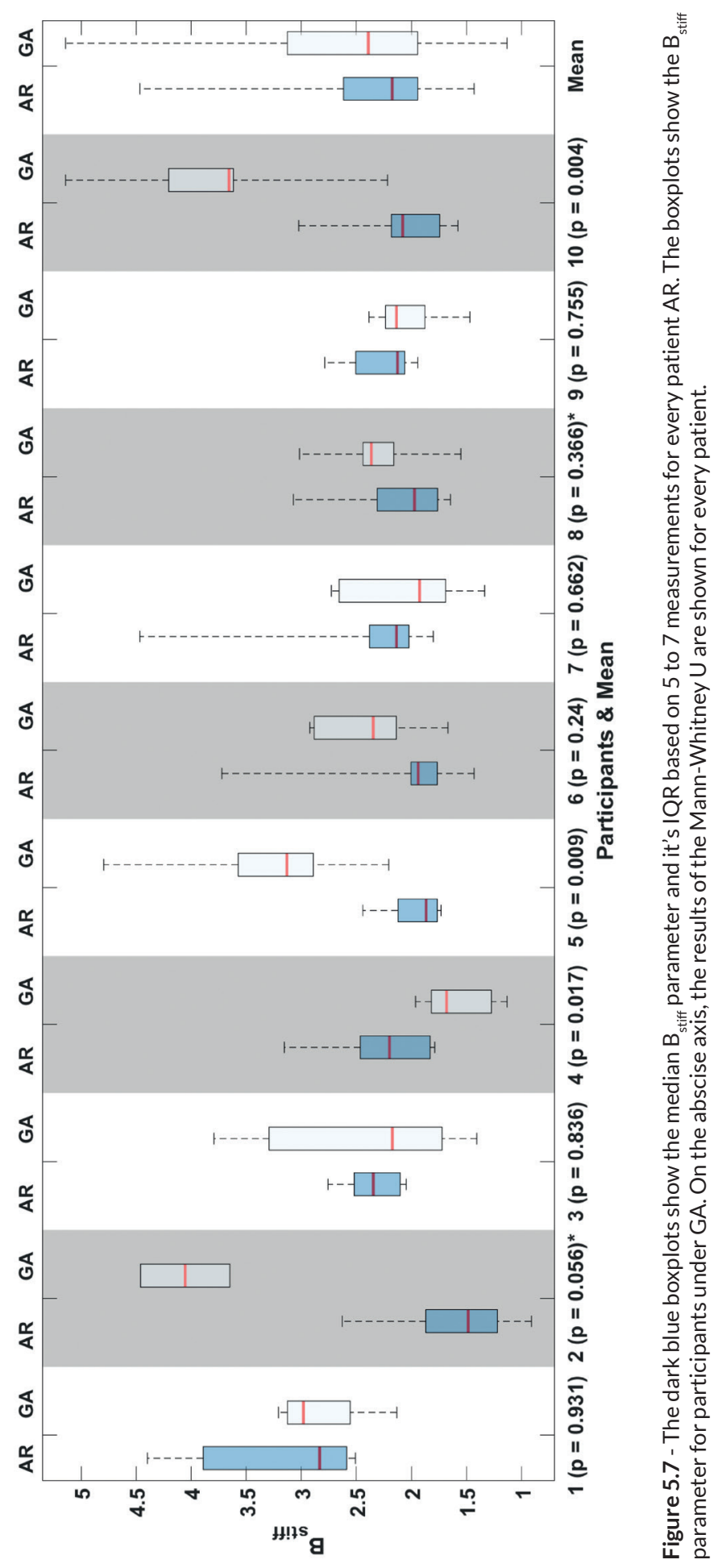


Table 5.3 - Stiffness parameter $A_{0.1}$ and stiffening parameter $\boldsymbol{B}_{\text {stiff }}$ of all patient combined and bovine measurements.

\begin{tabular}{|c|c|c|c|}
\hline \multirow{5}{*}{$\mathbb{8}^{50}$} & Test & $\mu \mathrm{M}$ & Normalized $\mu \mathrm{IQR}$ \\
\hline & Bovine muscle (ex-vivo) & $10185{\mathrm{~Pa} . \mathrm{S}^{-1}}^{-1}$ & $13 \%$ \\
\hline & At Rest & $1292 \mathrm{~Pa} \cdot \mathrm{S}^{-1}$ & $18 \%$ \\
\hline & under General Anesthesia & 3289 Pa.S-1 & $63 \%$ \\
\hline & Wilcoxon Signed-Rank (At Rest - under GA) & $p=0.002$ & \\
\hline \multirow{4}{*}{$\infty^{\frac{E^{5}}{5}}$} & Bovine muscle (ex-vivo) & 1.20 & $15 \%$ \\
\hline & At Rest & 2.10 & $12 \%$ \\
\hline & under General Anesthesia & 2.65 & $37 \%$ \\
\hline & Wilcoxon Signed-Rank (At Rest - under GA) & $P=0.19$ (not & \\
\hline
\end{tabular}

The initial Pressure-Shape slope $A_{0.1}$ is significantly higher (2.5 times) under GA than AR (Table 5.3, Figure 5.6). The normalized $\mu \mathrm{IQR}$ is $18 \%$ while $A R$, which is just above $13 \%$ obtained during the bovine ex-vivo measurement. Some of the measurements under GA show a lot of variation causing the normalized $\mu \mathrm{IQR}$ reaches to reach a value of $63 \%$. Nevertheless, the Mann-Whitney $U$ test confirms that in 8 out of 10 participants the measurements are significantly higher under GA than AR ( $p$-value lower than 0.05,Figure 5.6). Contrary to the $A_{0.1}$, the $\mu \mathrm{M}$ of the stiffening factor $B_{\text {stiff }}$ is not significantly different in both situations (Table 5.3, Figure 5.7). The normalized $\mu$ IQR under GA is lower for $B_{\text {Stiff }}$ then for $A_{0.1}$. Three and one measurement(s) were removed from participants 2 and 8, respectively. These measurements did not reach a shape value 0.5 , thus the ratio value $B_{\text {Stiff }}$ could not be evaluated for these measurements. 


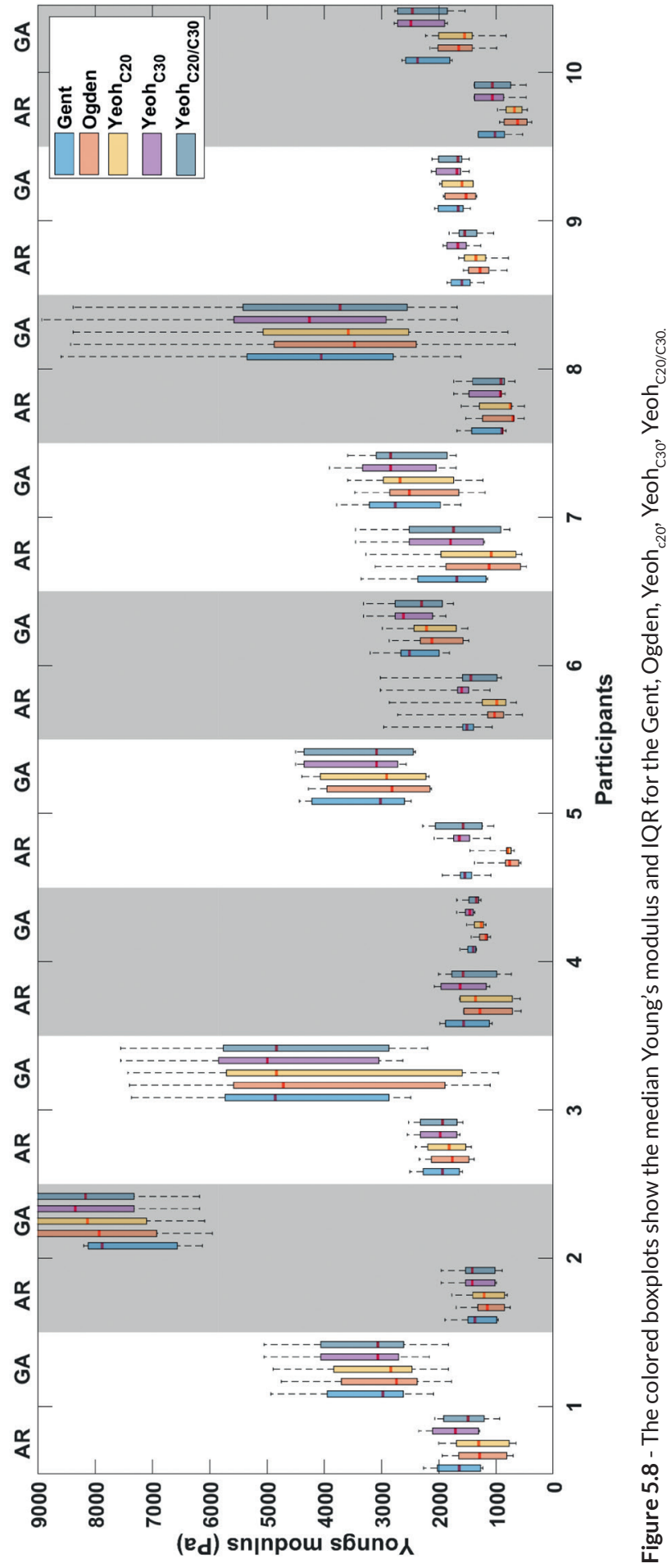




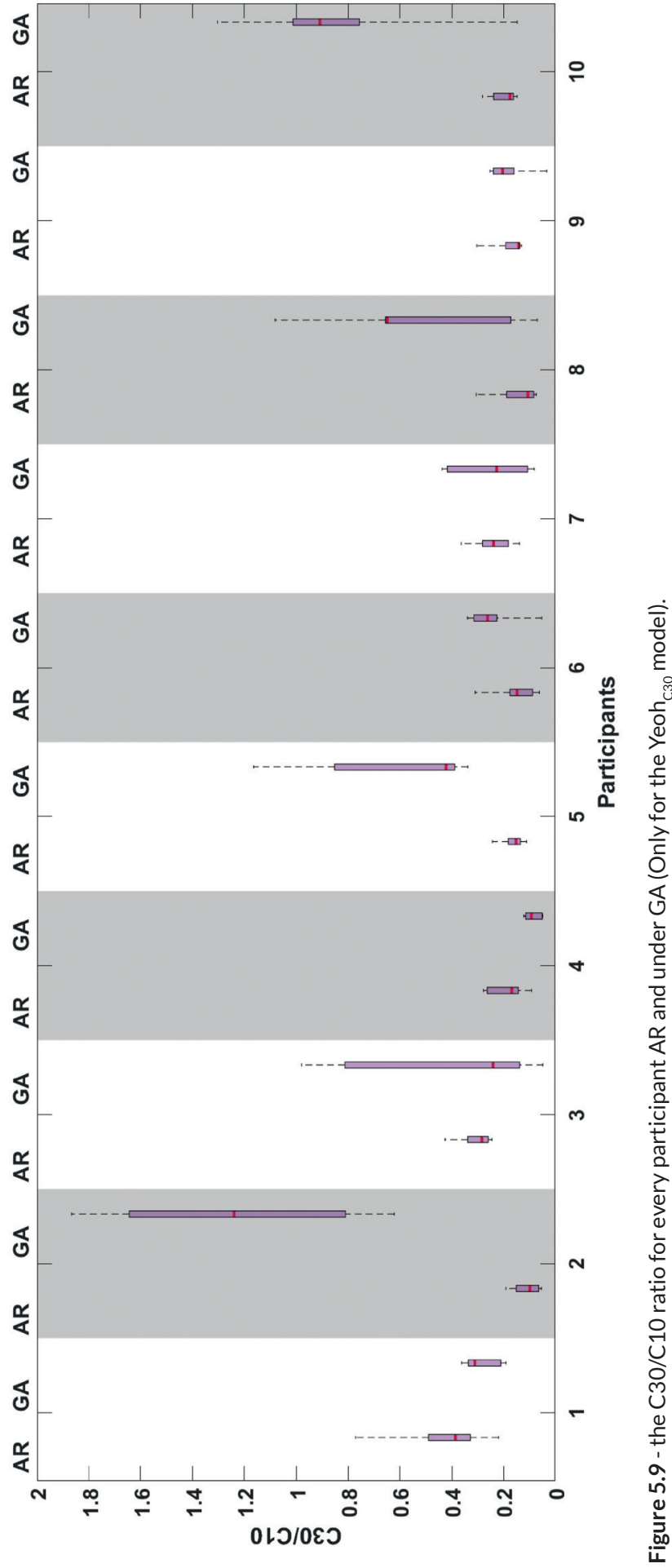


The identified Young's Moduli for every patient using different models from Table 5.1 are shown in Figure 5.8. The resemblance with $A_{0.1}$ from Figure 5.6 is striking: the median Young's Moduli differs from slope $A_{0.1}$ with a factor 0.88 (STD 0.11) to 1.14 (std 0.21) depending on the used constitutive model. The global fitting quality for all the models is similar $\left(\emptyset_{1}\right.$ values lower than 0.052$)$. The second part $\emptyset_{2}$ of the $\emptyset$ function, that resembles the capacity of the model to properly fit the Pressure-Shape slope at the start of the curve, differs for each model. Their average performances can be sorted as the following (best to worst median value): Yeoh $\mathrm{C20/c30}$ (0.0003), Yeoh ${ }_{\mathrm{c} 30}$ (0.0007), Gent (0.0012), Yeoh ${ }_{\mathrm{c} 20}(0.0012)$, Ogden (0.0014).

In biomechanical modeling, two-parameter models are usually used in literature. The mean Youngs modulus by the Yeoh $_{\mathrm{C} 30}$, the best performing two-parameter model, is $1638 \mathrm{~Pa}$ AR and 3060 under GA. The parameter ratio C30/C10 is shown in Figure 5.9 and provides a mean of 0.19 and 0.46 for all patients for AR and under GA, respectively.

The pressure-Shape curves (Figure 5.4b) have been computed for parameters of each model chosen in a range representative of the experimental data. The associated cumulated volume repartition curves $f\left(I_{1}-3\right)$ at specific shapes 0.2 , $0.4,0.6$ and 0.8 , have been computed using the volume $V_{\text {ref }}$ as a cylinder with a height and a radius of 0.75 aperture diameter.

\section{Measurement variabilities}

Table 5.4 - Parameter sensitivity analysis on the initial Pressure-Shape curve $A_{0.1}$.

\begin{tabular}{|c|c|c|c|c|}
\hline $\begin{array}{l}\text { Performed } \\
\text { test }\end{array}$ & $\begin{array}{l}\text { Reference } \\
\text { value } \boldsymbol{X}_{0}\end{array}$ & Parameter tested range & $\begin{array}{l}\text { Impact on Slope } \\
\text { variation } \Delta \boldsymbol{A}_{0.1} \\
\text { and std }\end{array}$ & $\begin{array}{l}\text { Error percentage if } \\
\text { slope } A_{0.1} \text { of } 1000 \\
{\boldsymbol{P a} . S^{-1}}\left(Y_{e o h}\right. \\
\text { C } 10=200 \mathrm{~Pa} \text {, } \\
\text { C } 30=10 \mathrm{~Pa}) .\end{array}$ \\
\hline $\begin{array}{l}\text { Average } \\
\text { Temperature } \\
\text { impact }\end{array}$ & $25^{\circ} \mathrm{C}$ & $\begin{array}{l}\text { Experimental test on } \\
\text { calibration curve: }\left[25^{\circ} \mathrm{C}\right. \\
\left.-37^{\circ} \mathrm{C}\right], 8 \text { measurements } \\
\text { each. Aperture } \\
\text { temperature average } \\
\text { stability in the mouth is } \\
\text { reached in less than } 60 \\
\text { seconds. }\end{array}$ & $30 \pm s t d 14.6$ Pa. $S^{-1}$ & $3 \pm s t d 1.5 \%$ \\
\hline $\begin{array}{l}\text { Friction } \\
\text { coefficient }\end{array}$ & 0.2 & $\begin{array}{l}\text { Numerical tested range: } \\
{[0.11]}\end{array}$ & 15.7 Pa. $S^{-1}$ & $1.6 \%$ \\
\hline $\begin{array}{l}\text { Poisson } \\
\text { ratio }\end{array}$ & 0.5 & $\begin{array}{l}\text { Numerical tested range } \\
{[0.4990 .5]}\end{array}$ & 5.6 Pa. $S^{-1}$ & $0.6 \%$ \\
\hline
\end{tabular}


A parameter sensitivity analysis is presented in Table 5.4. The impact on the initial Pressure-Shape curve slope is evaluated for specific parameter ranges. For

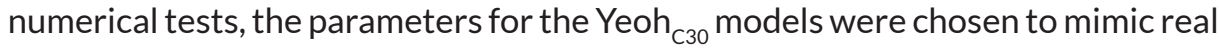
experimental data: $\mathrm{C} 10=200 \mathrm{~Pa}(\mathrm{E}=1200 \mathrm{~Pa}), \mathrm{C} 30=10 \mathrm{~Pa}$.

\subsection{DISCUSSION}

To our knowledge, this is the first study that measured and compared the tongue stiffness AR and under GA. This is also the largest cohort of individuals that participated in tongue stiffness measurements at the moment of publishing. The aspiration technique is non-invasive and quick, as a single measurement and analysis take up less than one minute. It can therefore be used almost in real-time, which was not possible using the previous version of the method [22,28].

\section{Slope, stiffness and stiffening differences between AR and under GA}

In this work, the initial slope $A_{0.1}$ and associated Young's Modulus of the tongue under GA are 2.5 and 1.9 times larger than AR, respectively. This difference is significant as shown in the Wilcoxon signed-rank test. On an individual level, the Mann-Whitney $U$ test showed that the difference between AR and under GA is significant for 8 out of the 10 participants. The assumption that muscle tone is absent or lower under GA is proven to be erroneous: results show an almost universal increase of tongue stiffness during anesthesia. Measurements AR thus provide a better reference state than under GA. These results are the opposite of what the authors expected and motivated extended experimental and numerical tests.

We found three plausible hypotheses in the literature to explain these results:

1) Sufentanil and Remifentanil, drugs administered during GA to all participants of this study, are reported to cause chest wall rigidity among other lipophilic opioids [41-44]. The frequently administered Rocuronium, a neuromuscular blocking agent, could counteract these effects by causing paralysis of skeletal muscle. However, few studies can be found about the interaction of the combined pharmaceuticals during anesthesia for muscle activation, and for the tongue in particular [45]. Analysis of the administrated pharmaceuticals could not fully explain the exceptions of the two participants (4 and 9) who showed an identical stiffness under GA and AR.

2) Upper airway reflexes, that can be activated under GA by intubation, are not always completely suppressed during general anesthesia [46]. Such (remnants of) reflexes are unequally pronounced among humans and depend on the depth of anesthesia, which could explain the observed patient-specific singular 
absence of stiffening in participant 4 and 9 [47]. However, this is hard to confirm since to our knowledge, no EMG measurements have ever been performed to assess muscle tone during anesthesia at the base of the tongue.

3) The lack of movements and administered drugs under GA alter blood flow and perfusion and could in turn affect the identified rigidity $[48,49]$. Manually palpating tongue tissue under GA and AR, unfortunately, did not provide a subjective correlated observation as measurements took place days apart. The tongue under GA also showed a more persisting tissue deformation after aspiration. The tissue deformation was resolved quicker by palpating the tongue.

The stiffening ratio $B_{\text {stiff }}$ shows that the slope was more than doubled from shape 0.1 to 0.5 (Figure 5.7, Eq 5.3). This effect is translated into a C30/C10 ratio for the Yeoh $_{\mathrm{C} 30}$ model (Figure 5.9): the median $\mathrm{C} 30 / \mathrm{C} 10$ ratio value is in the range of [0.1 - 0.5$]$ for $70 \%$ of participants both under GA and AR. This ratio range can be implemented in numerical models when assuming a perfectly incompressible material. The ratio $\mathrm{C} 30 / \mathrm{C} 10$ can reach values as high as 1.8 but the patientspecific high values remained unexplored.

\section{Measurement uncertainties}

Given the unexpected results for the observed experimental difference between $A R$ and under GA, the measurement method has been challenged with different phenomena using the initial slope $A_{0.1}$ as criteria (Table 5.4).

\section{Variability of experimental slope A0.1}

The reproducibility of the method on biological tissues has been experimentally evaluated during the bovine meat ex-vivo test, assuming stable material parameters and a comparable situation with in-vivo testing (tissue compressibility, friction coefficient, load, temperature, etc): the normalized $\mu \mathrm{LQR}$ of ex-vivo bovine meat is $13 \%$ of the median initial slope $A_{0.1}$. The reproducibility limitations of the method itself can thus not account for normalized $\mu \mathrm{IQR}$ of $18 \%$ and $63 \%$ for the patient measurements (Table 5.3). The variability during the measurements AR could be caused by small uncontrolled tongue movements or breathing. During $\mathrm{GA}$, the often cumbersome accessibility of the tongue made replacing the suction cup at the same place difficult and, therefore, less reproducible. Also, phenomena explained in the previous paragraph could induce a change in material stiffness between or during measurements.

An average temperature difference in the mouth between AR and under GA is expected due to breathing or because the patient is intubated. In the conducted immersion test an average temperature difference as high as $12^{\circ} \mathrm{C}$ surrounding 
the cup (immersed in $37^{\circ} \mathrm{C}$ or $25^{\circ} \mathrm{C}$ room temperature water) proved to have a negligible effect on the initial slope $A_{0.1}(3 \pm s t d 1.5 \%$, Table 5.4).

A residual load, exercised by the weight of the suction cup and the tube, was present at both the measurements AR and under GA. The normal load contribution of the cup to the surface induced by the tube has been measured with a precision balance and was found to be lower than one gram, which is considered negligible in respect to other devices used in literature [22,50], or commercial products such as the Cutometer (Courage and Khazaka, Köln, Germany).

\section{Parameter influencing the extracted Young's Modulus}

The aspiration technique and method have been validated on non-stiffening silicon objects in Elahi et al. [27,28]. It provided results with a maximum error of $+8.8 \%$ compared to classical tests such as tensile or bulge tests. It was verified experimentally that the adjustments made in the measurement setup and analysis provided similar results and identification range.

Identifying parameters for an in-vivo stiffening material yet presents more challenges: the Ogden, Gent, and Yeoh model formulations (Table 5.1) are only able to approximate the real material stiffening behavior (model misspecification). This leads to drastically different equivalent Young's moduli if only the shape distance is minimized ( $\varnothing=\emptyset_{1}, \beta=0$, Eq 5.4), even for satisfying global fitting results (results not presented). A value $\beta \in[0.10 .9]$ can, however, provide almost model-independent Young's Moduli (Figure 5.8, $\beta=0.5$ ) for satisfying fitting (example Figure 5.2). The independency of the Identified Young's Modulus to the model formulation was considered a validation of the inverse method and chosen minimized function.

The chosen minimized function $\emptyset$ provides material parameters for which the Pressure-Shape initial slope $A_{0.1}$ is respected thanks to the part $\emptyset_{2}$ of the minimized function (Eq 5.4). Obtaining a Young's Modulus almost proportional to the initial slope $A_{0.1}$ is thus an expected result.

Poisson and friction coefficient effects on the Pressure-Shape curve initial slope $A_{0.1}$ are below $1.6 \%$ and are considered negligible for the tested ranges compared to experimental reproducibility.

\section{Young's modulus identification \& literature comparison}

The Yeoh $_{\mathrm{C} 30}$ was the best performing two-parameter model in this study and is therefore compared to other literature. The median Young's modulus from all participants is $1638 \mathrm{~Pa}$ (min 1035 - max 2019) for AR and $3060 \mathrm{~Pa}$ (min 1477 max 8930) for under GA. 
The identified Young's modulus AR is 2.4 times smaller than the AR measurement of Schiavone et al. [22] with a Yeoh ${ }_{\mathrm{C} 30}$ model and $30 \%$ smaller than the ex-vivo cadaver measurement of Gerard et al. [15] with a Yeoh ${ }_{\text {c20 }}$ model (Table 5.5). Both the Young's modulus AR and under GA are more than 2 times smaller than invivo MRE [21] and 8 to 16 times smaller than the "activated measurement" of Schiavone et al. [22]. Aside from Cheng et al. [21], all results of the cited in-vivo studies were obtained only from one participant.

Young's Moduli measured in-vivo (Table 5.5), injected into biomechanical models, usually prove too stiff to simulate realistic tissue deformations $[10,11,16]$. This is substantiated by Hermant et al. [14] who simulated the tongue movements in the direction of the pharyngeal wall using both a common tissue stiffness for tongue models $(E=6222 \mathrm{~Pa})$ and a lower stiffness $(E=1116 \mathrm{~Pa})$ based on the exvivo experiments from Gerard et al. [15]. They found much more realistic behavior using the lower stiffness. The identified Young's Modulus range obtained of the ten measured participants in this work corroborates these results.

\section{Limitations and Perspectives}

To the authors' knowledge, no artificial material presents a mechanical behavior close to that of the tongue (Young's modulus and stiffening rate). This prevents the definition of a real gold standard to perform a fair comparison of identified Young's Modulus for the whole method using aspiration or classical testing. The present method could only be validated on artificial non-stiffening material $[27,28]$. However, the identification of a similar Young's modulus on different material models makes the model more trustworthy.

Figure 5.4b shows that the numerical Pressure-Shape curves are almost independent (for the simulated parameters) of the model for a Shape range of [0 $0.2]$. This shape range is associated with $\left(I_{1}-3\right)$ values in a range of [0 0.5] over the tissue volume Figure 5.4c), which in a tensile test would provide a principal deformation of $46 \%$. For larger deformations, the material models describe the material stiffening-strain relation differently. Local stiffening induces strain locking; the deformation is spread to less stressed material parts. The predicted volumetric repartition thus differs from the models for shapes greater than 0.4 (Figure 5.4c). The most physically relevant model cannot be selected according to this criterion given that the experimental strain distribution is unknown. 


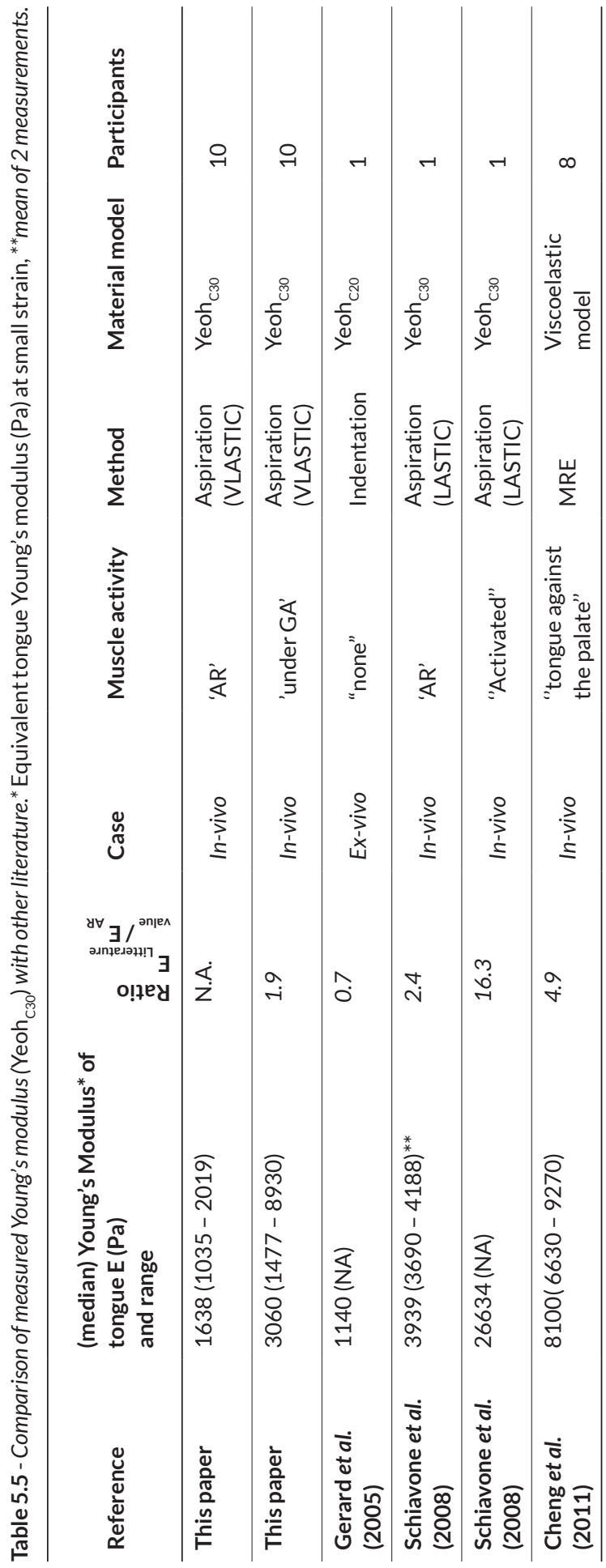


The obtained Pressure-Shape curves do not provide insight into all the tongue tissue properties (nonlinear, time-dependent, inhomogeneous, and anisotropic material). Furthermore, only one location of the tongue was measured using a suction cup with a fixed diameter. The use of different cup sizes in future research could enable us to measure tissue over larger and deeper areas but can be restricted by tongue boundary conditions.

As many factors influence the tongue stiffness under GA, a more controlled protocol is required to explain why tissue is stiffer under GA than AR. The three plausible hypotheses mentioned in the discussion could be tested. For example, EMG measurements of the tongue could give insight into the muscle activity during anesthesia. Also, monitoring the administration of medicine-only one by one, and in particular, a higher dose of Rocuronium, propofol, and fentanyl could provide insight into the effect of medicines on the tongue stiffness. Identifying the factors that lead to these surprising results could give insight on how to define a better reference state for the tongue. However, the clinical significance would be small for such an invasive study to be performed on humans.

Future research could focus on classifying fibrotic changes over time and between patients after surgery and/or radiation. This could be useful in studies focused on therapies to counteract fibrotic stiffening of tissue or to create more realistic biomechanical models that can incorporate fibrotic properties to improve the prediction of function loss [11,51]. Because this new technique enables stiffness measurements in a sterile environment, it can also be translated into other medical fields.

\subsection{CONCLUSION}

The stiffness of the tongue in 10 participants was measured AR and while under GA during surgery. While aiming to measure the stiffness of the tongue without muscle tone, the tongue unexpectedly appeared to be approximately 2 to 2.5 times stiffer under GA. While several hypotheses can potentially explain this behavior, no definitive answer could be provided with the current data. Between patients, the measurements AR were comparable and proved to be as reproducible as exvivo measurements using the same method. However, this did not apply to the measurements under GA. Identification using a two-parameter Yeoh constitutive model showed that the Young's modulus of the tongue in the relaxed condition $\mathrm{AR}$ is approximately $1638 \mathrm{~Pa}$ and the $\mathrm{C} 30 / \mathrm{C} 10$ ratio 0.19 . 


\subsection{REFERENCES}

1. Kreeft AM, Van Der Molen L, Hilgers FJ, Balm AJ. Speech and swallowing after surgical treatment of advanced oral and oropharyngeal carcinoma: A systematic review of the literature. Eur Arch Oto-Rhino-Laryngology. Springer; 2009;266: 1687-1698. doi:10.1007/s00405-009-1089-2

2. Van Der Molen L, Van Rossum MA, Jacobi I, Van Son RJJH, Smeele LE, Rasch CRN, et al. Pre- and posttreatment voice and speech outcomes in patients with advanced head and neck cancer treated with chemoradiotherapy: Expert listeners' and patient's perception. J Voice. Elsevier Ltd; 2012;26: 664.e25-664.e33. doi:10.1016/j.jvoice.2011.08.016

3. Costa Bandeira AK, Azevedo EHM, Vartanian JG, Nishimoto IN, Kowalski LP, CarraraDe Angelis E. Quality of life related to swallowing after tongue cancer treatment. Dysphagia. 2008;23: 183-192. doi:10.1007/s00455-007-9124-1

4. Lee DY, Ryu YJ, Hah JH, Kwon TK, Sung MW, Kim KH. Long-term subjective tongue function after partial glossectomy. J Oral Rehabil. 2014;41: 754-758. doi:10.1111/ joor.12193

5. Husaini H, Krisciunas GP, Langmore S, Mojica JK, Urken ML, Jacobson AS, et al. A survey of variables used by speech-language pathologists to assess function and predict functional recovery in oral cancer patients. Dysphagia. 2014;29:376-386. doi:10.1007/ s00455-014-9520-2

6. Montero PH, Patel SG. Cancer of the oral cavity. Surg Oncol Clin N Am. 2015/04/15. 2015;24: 491-508. doi:10.1016/j.soc.2015.03.006

7. Sessions DG, Spector GJ, Lenox J, Haughey B, Chao C, Marks J. Analysis of treatment results for oral tongue cancer. Laryngoscope. 2002;112: 616-25. doi:10.1097/00005537-200204000-00005

8. Shah JP, Gil Z. Current concepts in management of oral cancer - Surgery. Oral Oncol. 2009;45: 394-401. doi:10.1016/j.oraloncology.2008.05.017

9. Fujita S, Dang J, Suzuki N, Honda K. A Computational Tongue Model and its Clinical Application. Oral Sci Int. 2007;4: 97-109. doi:10.1016/S1348-8643(07)80004-8

10. Buchaillard S, Brix M, Perrier P, Payan Y. Simulations of the consequences of tongue surgery on tongue mobility: Implications for speech production in post-surgery conditions. Int J Med Robot ComputAssist Surg. 2007;3: 252-261. doi:10.1002/rcs.142

11. Kappert KDR, van Alphen MJA, van Dijk S, Smeele LE, Balm AJM, van der Heijden F. An interactive surgical simulation tool to assess the consequences of a partial glossectomy on a biomechanical model of the tongue. Comput Methods Biomech Biomed Engin. 2019;22: 827-839. doi:10.1080/10255842.2019.1599362

12. Vogt F, Lloyd JE, Buchaillard S, Perrier P, Chabanas M, Payan Y, et al. Efficient 3D finite element modeling of a muscle-activated tongue. Lect Notes Comput Sci. 2006; 19-28. doi:10.1007/11790273_3

13. Van Alphen MJA, Kreeft AM, Van Der Heijden F, Smeele LE, Balm AJM. Towards virtual surgery in oral cancer to predict postoperative oral functions preoperatively. Br J Oral Maxillofac Surg. British Association of Oral and Maxillofacial Surgeons; 2013;51: 747751. doi:10.1016/j.bjoms.2013.06.012

14. Hermant N, Perrier P, Payan Y. Human Tongue Biomechanical Modeling. In: Payan Y, Ohayon JBT-B of LO, editors. Biomechanics of Living Organs: Hyperelastic Constitutive Laws for Finite Element Modeling. Oxford: Academic Press; 2017. pp. 395-411. doi:10.1016/B978-0-12-804009-6.00019-5 
15. Gerard JMM, Ohayon J, Luboz V, Perrier P, Payan Y. Non-linear elastic properties of the lingual and facial tissues assessed by indentation technique: Application to the biomechanics of speech production. Med Eng Phys. Elsevier; 2005;27: 884-892. doi:10.1016/j.medengphy.2005.08.001

16. Buchaillard S, Perrier P, Payan Y. A biomechanical model of cardinal vowel production: muscle activations and the impact of gravity on tongue positioning. J Acoust Soc Am. 2009;126: 2033-2051. doi:10.1121/1.3204306

17. Stavness I, Lloyd JE, Fels S. Automatic prediction of tongue muscle activations using a finite element model. J Biomech. Elsevier; 2012;45: 2841-2848. doi:10.1016/j. jbiomech.2012.08.031

18. Wilhelms-Tricarico R. Physiological modeling of speech production: methods for modeling soft-tissue articulators. J Acoust Soc Am. United States, United States; 1995;97: 3085-3098. doi:10.1121/1.411871

19. Wang YK, Nash MP, Pullan AJ, Kieser JA, Rohrle O. Model-based identification of motion sensor placement for tracking retraction and elongation of the tongue. Biomech Model Mechanobiol. Germany, Germany; 2013;12:383-399. doi:10.1007/s10237-012-0407-6

20. Pelteret JPV, Reddy BD. Computational model of soft tissues in the human upper airway. Int J Numer Method Biomed Eng. England; 2012;28: 111-132. doi:10.1002/cnm.1487

21. Cheng S, Gandevia SC, Green M, Sinkus R, Bilston LE. Viscoelastic properties of the tongue and soft palate using MR elastography. J Biomech. Elsevier; 2011;44: 450-454. doi:10.1016/j.jbiomech.2010.09.027

22. Schiavone P, Boudou T, Promayon E, Perrier P, Payan Y. A light sterilizable pipette device for the in vivo estimation of human soft tissues constitutive laws. 2008 30th Annual International Conference of the IEEE Engineering in Medicine and Biology Society. IEEE; 2008. pp. 4298-4301. doi:10.1109/IEMBS.2008.4650160

23. Clayton EH, Garbow JR, Bayly P V. Frequency-dependent viscoelastic parameters of mouse brain tissue estimated by MR elastography. Phys Med Biol. 2011;56: $2391-$ 2406. doi:10.1088/0031-9155/56/8/005

24. Kerdok AE, Ottensmeyer MP, Howe RD. Effects of perfusion on the viscoelastic characteristics of liver. J Biomech. United States, United States; 2006;39: 2221-2231. doi:10.1016/j.jbiomech.2005.07.005

25. Gefen A, Margulies SS. Are in vivo and in situ brain tissues mechanically similar? J Biomech. United States, United States; 2004;37: 1339-1352. doi:10.1016/j. jbiomech.2003.12.032

26. Fung YCB. Biorheology of soft tissues. Biorheology. 1973;10: 139-155. doi:10.3233/ BIR-1973-10208

27. Elahi SA, Connesson N, Payan Y. Disposable systems for in-vivo mechanical characterization of soft tissue based on volume measurement. J Mech Med Biol. World Scientific Pub Co Pte Lt; 2018;18. doi:10.1142/S0219519418500379

28. Elahi SA, Connesson N, Chagnon G, Payan Y. In-vivo soft tissues mechanical characterization: volume-vased vspiration method validated on silicones. Exp Mech. 2019;59: 251-261. doi:10.1007/s11340-018-00440-9

29. Horgan CO. The remarkable Gent constitutive model for hyperelastic materials. Int J Non Linear Mech. 2015;68: 9-16. doi:10.1016/j.jjnonlinmec.2014.05.010

30. Yeoh $\mathrm{OH}$. Some forms of the strain energy function for rubber. Rubber Chem Technol. 1993;66: 754-771. doi:10.5254/1.3538343 
31. Ogden R. Non-linear elastic deformations. Eng Anal Bound Elem. 1984;1: 119. doi:10.1016/0955-7997(84)90049-3

32. Rashid B, Destrade M, Gilchrist MD. Mechanical characterization of brain tissue in compression at dynamic strain rates. J Mech Behav Biomed Mater. 2012;10: 23-38. doi:10.1016/j.jmbbm.2012.01.022

33. Budday S, Sommer G, Birkl C, Langkammer C, Haybaeck J, Kohnert J, et al. Mechanical characterization of human brain tissue. Acta Biomater. 2017;48: 319-340. doi:10.1016/j.actbio.2016.10.036

34. Mehrabian H, Samani A. An iterative hyperelastic parameters reconstruction for breast cancer assessment. Medical Imaging 2008: Physiology, Function, and Structure from Medical Images. 2008. doi:10.1117/12.770971

35. Sadeghnejad S, Elyasi N, Farahmand F, Vossughi GR, Sadr Hosseini SM. Hyperelastic modeling of sino-nasal tissue for haptic neurosurgery simulation. Sci Iran. 2019;27: 1266-1276. doi:10.24200/sci.2019.50348.1652

36. Schiavone P, Chassat F, Boudou T, Promayon E, Valdivia F, Payan Y. In vivo measurement of human brain elasticity using a light aspiration device. Med Image Anal. 2009;13: 673678. doi:10.1016/j.media.2009.04.001

37. Schiavone P, Promayon E, Payan Y. LASTIC: A Light Aspiration Device for in vivo Soft TIssue Characterization. Lecture Notes in Computer Science (including subseries Lecture Notes in Artificial Intelligence and Lecture Notes in Bioinformatics). 2010. pp. 1-10. doi:10.1007/978-3-642-11615-5_1

38. Lang YD, Malacina A, Biegler LT, Munteanu S, Madsen JI, Zitney SE. Reduced order model based on principal component analysis for process simulation and optimization. Energy and Fuels. 2009. pp. 1695-1706. doi:10.1021/ef800984v

39. Mann HB, Whitney DR. On a Test of Whether one of Two Random Variables is Stochastically Larger than the Other. Ann Math Stat. 1947;18: 50-60. doi:10.1214/ aoms/1177730491

40. Wilcoxon F. Probability Tables for Individual Comparisons by Ranking Methods. Biometrics. 1947;3: 119. doi:10.2307/3001946

41. Ackerman WE, Phero JC, Theodore GT. Ineffective ventilation during conscious sedation due to chest wall rigidity after intravenous midazolam and fentanyl. Anesth Prog. 1990;37: 46-8. Available: https://www.ncbi.nlm.nih.gov/pubmed/2077987

42. Buxton JA, Gauthier T, Kinshella M-LW, Godwin J. A 52-year-old man with fentanylinduced muscle rigidity. CMAJ. Joule Inc.; 2018;190: E539-E541. doi:10.1503/ cmaj.171468

43. Dimitriou V, Zogogiannis I, Liotiri D, Wambi F, Tawfeeq N, Koumi A, et al. Impossible mask ventilation after an unusually low dose fentanyl-induced muscle rigidity in a patient with essential tremor: A case report and review of the literature. Middle East J Anesthesiol. Lebanon, Lebanon; 2014;22: 619-622.

44. Müller P, Vogtmann C. Three cases with different presentation of fentanyl-induced muscle rigidity - A rare problem in intensive care of neonates. Am J Perinatol. United States, United States; 2000;17: 23-26. doi:10.1055/s-2000-7289

45. Nakada J, Nishira M, Hosoda R, Funaki K, Takahashi S, Matsura T, et al. Priming with rocuronium or vecuronium prevents remifentanil-mediated muscle rigidity and difficult ventilation. J Anesth. 2009;23: 323-328. doi:10.1007/s00540-009-0769-9 
46. Tagaito Y, Isono S, Nishino T. Upper airway reflexes during a combination of propofol and fentanyl anesthesia. Anesthesiology. 1998;88: 1459-1466. doi:10.1097/00000542199806000-00007

47. Davies AE, Stone SP, Kidd D, MacMahon J. Pharyngeal sensation and gag reflex in healthy subjects. Lancet. 1995;345: 487-488. doi:10.1016/S0140-6736(95)90584-7

48. Akata T. General anesthetics and vascular smooth muscle: Direct actions of general anesthetics on cellular mechanisms regulating vascular tone. Anesthesiology. 2007. pp. 365-391. doi:10.1097/00000542-200702000-00026

49. Schwarte L, Loer S, Schober P. Effects of thiopental and propofol on skeletal muscle oxygenation. Eur J Anaesthesiol. 2008;25: 60. doi:10.1097/00003643-200805001-00186

50. Nava A, Mazza E, Furrer M, Villiger P, Reinhart WH. In vivo mechanical characterization of human liver. Med Image Anal. 2008;12: 203-216. doi:10.1016/j.media.2007.10.001

51. Kappert KDR, van Alphen MJA, Smeele LE, Balm AJM, van der Heijden F. Quantification of tongue mobility impairment using optical tracking in patients after receiving primary surgery or chemoradiation. Kimple RJ, editor. PLoS One. 2019;14: e0221593. doi:10.1371/journal.pone.0221593 


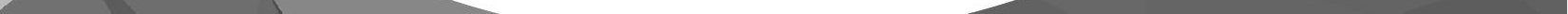




\title{
PERSONALIZED
}

\section{BIOMECHANICAL TONGUE}

MODELS BASED ON

DIFFUSION-WEIGHTED MRI

AND VALIDATED USING

OPTICAL TRACKING OF

RANGE OF MOTION.

\author{
K. D.R. Kappert \\ L. Voskuilen \\ L.E. Smeele \\ A.J.M. Balm \\ B. Jasperse \\ A.J. Nederveen \\ F. van der Heijden
}

This chapter is resubmitted after major revisions to Biomechanics and Modeling in Mechanobiology

This work was presented at the Technical Innovations In Medicine congress, Webinar (2020) 


\section{ABSTRACT}

For advanced tongue cancer, the choice between surgery or organ-sparing treatment is often dependent on the expected loss of tongue functionality after treatment. Biomechanical models might assist in this choice by simulating the post-treatment function loss. However, this function loss varies between patients and should, therefore, be predicted for each patient individually. In the present study, the goal was to better predict the postoperative range-of-motion (ROM) of the tongue by personalizing biomechanical models using diffusion-weighted MRI and constrained spherical deconvolution (CSD) reconstructions of tongue muscle architecture.

Diffusion-weighted MRI scans of ten healthy volunteers were obtained to reconstruct their tongue musculatures, which were subsequently registered to a previously described population average or atlas. Using the displacement fields obtained from the registration, the segmented muscle fiber tracks from the atlas were morphed back to create personalized muscle fiber tracks. Finite element models were created from the fiber tracks of the atlas and those of the individual tongues. Via inverse simulation of a protruding, downward, left and right movement, the ROM of the tongue was predicted. This prediction was compared to the ROM measured with a 3D camera.

For the personalized biomechanical models, $80 \%$ of the movements were predicted correctly whereas, for the generic model, only $50 \%$ of the movements were predicted correctly.

In conclusion, personalized biomechanical models of the tongue better predict tongue ROM and may in the future improve the estimation of function loss after treatment for advanced tongue carcinoma. 


\subsection{INTRODUCTION}

The incidence of tongue cancer is rising worldwide, accounting for almost $20 \%$ of all head and neck cancers [1,2]. Locally advanced tongue cancer is usually treated by surgery and/or chemoradiation, which may have a serious impact on the mobility of the tongue due to surgical defects and/or radiation-induced fibrosis. This often leads to difficulties with speech, mastication, and swallowing $[3,4]$. The choice between surgical or organ-sparing treatment is dependent on expected function loss after treatment, which is difficult to predict [5]. The prediction of the expected function loss would be of great benefit for the decision-making process shared between physician and patient. Biomechanical modeling of the tongue would be a logical next step in the process of the prediction of functional loss.

The biomechanics of the tongue however are complex [5-7]. The tongue consists of four extrinsic and four intrinsic muscles, which interdigitate and seem to follow a strict pattern [8]. Although we know that all muscles, except for the palatoglossus muscle, are innervated by the hypoglossal nerve $[9,10]$, the complex neural strategies that are required for shaping the tongue during speech and mastication, are currently unknown [11,12]. Moreover, the tongue shape varies between individuals, and knowledge about anatomical variations in muscle structure does not yet exist [13]. Although biomechanical finite-element (FE) models have shown to be a promising method to predict functional loss after treatment [14-16], these models have often been created from ex-vivo data, and are generally generic. These FE models are therefore unable to predict functional loss on an individual level and should be personalized.

One way of creating personalized FE models is by morphing of a generic FE model to a subject-specific situation $[17,18]$. Previous work has shown that this morphing can be driven by imaging data such as anatomical slices [19], computed tomography [20,21], and MRI [22,23]. Alternatively, personalized models can also be constructed by embedding mesh and muscle structures in a coarse FE model that is generated according to the shape of the mesh. [24,25]. If muscles are however included in personalized models, the morphing should not only be driven by the outline of anatomical structures or meshes, but also the internal structure of the muscle, such as the muscle fiber directionality.

This muscle fiber directionality can be measured by exploiting the possibilities of diffusion-weighted MRI [26]. Using diffusion-sensitizing gradients, it is possible to encode MR images with diffusion information along a certain direction. As the diffusivity of water is higher along muscle fibers than perpendicular to them, it is possible to reconstruct the fiber orientation using the diffusion tensor. In fiber tracking or tractography, fiber tracks are computed from these fiber orientations, 
[27], easing the visualization of the tongue musculature [28-31]. These tracks have even been used as an input for biomechanical models of the tongue [32]. Despite this potential of DTI, it is unable to resolve the interdigitating muscle fibers on the tongue. Recently, a diffusion-weighted MRI technique called constrained spherical deconvolution (CSD), which can resolve the interdigitating muscle fibers of the tongue in vivo, was applied to the tongue [33]. This technique enables us to reconstruct the tongue muscle architecture of the individual more accurately.

The goal of the current work was to create personalized biomechanical models of the tongue by using CSD MRI. As manual embedding of all the fibers of this muscle architecture in the FE model would be very laborious, it is hardly feasible. This motivated us to use automated methods to embed these fibers. However, due to the high noise levels and artifacts in CSD MRI, the quality of reconstructed musculature of a single subject was relatively low. We, therefore, proposed to use a population average or atlas of the tongue muscle architecture [34], which was more resistant to noise and artifacts. By registering the muscle architecture of an individual to that of the atlas, we hypothesized that the segmented fiber tracks of the atlas can be morphed back to an individual's space and, subsequently, from these segmented fiber tracks a personalized biomechanical model can be created. These models were validated by comparing the predicted range-of-motion (ROM) of the tongue with the ROM measured in vivo using 3D optical tracking [35].

\subsection{METHODS}

The following section covers the characteristics of volunteers and the measurement of their ROM. Next, the creation of the personalized biomechanical models is described, which is summarized in Figure 6.1. Methods are divided into those applied to the atlas (Figure 6.1 A1-9) and those applied to personalized models (Figure $6.1 \mathrm{P} 1-7)$. Finally, the ROM predicted by these biomechanical models and the atlas were compared to the measured ROM.

\section{Volunteers \& ROM measurement}

A total of ten healthy volunteers were included with a mean age of 60 years (range: 50 to 71; seven men) to match the same age group of tongue cancer patients. Volunteers with steel braces or any contra-indication to an MRI scan were excluded.

The ROM of the tongue was obtained by optical tracking of a marker on the tip of the tongue using a 3D camera. The volunteers were asked to perform five different tongue movements: left, right, down, and protrusion as described in the paper by Kappert et al. [35]. Written informed consent was obtained from 
all volunteers before inclusion. This study was approved by the medical ethical committee of the Netherlands Cancer Institute (ref: N17BTM).

\section{CSD MRI acquisition and processing}

The volunteers were scanned in a 3 T Philips MRI scanner (Philips Healthcare, Best, The Netherlands) using a neurovascular coil according to the CSD scan protocol by Voskuilen et al. [33] (Figure 6.1 P1). The raw diffusion-weighted images were acquired using the following parameters: single-shot spin-echo echo-planar imaging; echo-train length 25; repetition time: $3.4 \mathrm{~s}$; echo time: 60 ms; two repetitions with opposing phase-encoding directions; number of signal averages: 1; fat suppression: spectral presaturation with inversion recovery and slice-selection gradient reversal; field-of-view: 192 by 156 by $84 \mathrm{~mm}$; voxel size: $3 \mathrm{~mm}$ isotropic; b-value: $700 \mathrm{~s} / \mathrm{mm}^{2}$ along 64 directions evenly spaced over a hemisphere and optimized for gradient load; total scan time: 10 minutes.

Subsequently, the diffusion-weighted images were denoised [36]. In FSL [37], the diffusion-weighted images were corrected for distortions caused by $\mathrm{B}_{0}$ inhomogeneity, eddy currents from the diffusion-encoding gradients, and rigid motion [38]. For all subjects, masks of the tongue were created by manual delineation in ITK-Snap [39]. In MRtrix3 [40], the corrected diffusion-weighted images were upsampled to a resolution of $1.5 \mathrm{~mm}$ isotropic using a b-spline interpolation. For each volunteer, a response function was estimated that corresponds to a single fiber population [41]. By deconvolving, using CSD, the corrected diffusion-weighted images with this response function [42], fiberorientation distribution (FOD) maps were calculated up to a maximum spherical harmonic degree of 8 (Figure 6.1 P2).

Finally, using symmetric diffeomorphic registration based on the FOD maps [43], displacement fields were calculated from each volunteer to the tongue muscle atlas described in Voskuilen et al. [34] (Figure 6.1 P3). This atlas is a population average of ten volunteers different than those included in this study (mean age of 25.5 years; four female). The difference between the registered FOD maps and the atlas was quantified by the $\mathrm{L}_{2}$-norm and the angular correlation coefficient [44]. The calculated displacement fields were used later in this work to morph the generical biomechanical model of the atlas. 


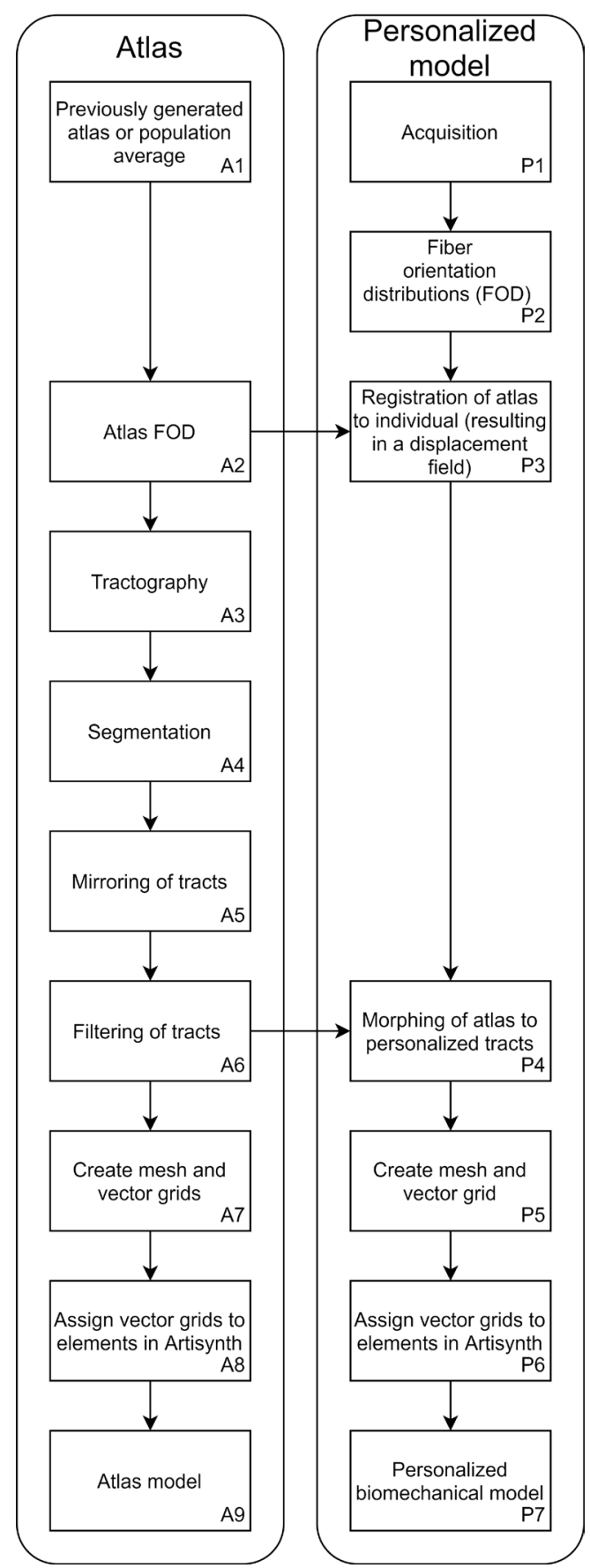

Figure 6.1 - A flow chart of the steps required to create an atlas-based (A1-9) and a personalized model (P1-7) 


\section{Fiber tracking and filtering}

Although it would be possible to compute a 3D vector field (required to build a biomechanical model) from the FOD maps directly, CSD-based fiber tracking was first performed on the atlas [45] (Figure 6.1 A3). Fiber tracking ignored many spurious vectors, and the segmentation of streamlines was less timeconsuming that segmentation of vectors. For this global fiber tracking, the following parameters were used: step size: $1.5 \mathrm{~mm}$; angular threshold: $15^{\circ}$; FOD peak threshold: $10 \%$ of the largest peak; maximal length: $100 \mathrm{~mm}$; minimal length: $10 \mathrm{~mm}$; number of seed points: 10,000 randomly placed within the mask. In TrackVis [46], the fiber tracking was manually segmented into the following muscle tracts: genioglossus, geniohyoid, hyoglossus, inferior longitudinal, superior longitudinal, transverse, and vertical muscles (Figure 6.1 A4 and Figure 6.2). These segmentations were subsequently checked by a head-and-neck surgeon. The styloglossus muscle could not be distinguished from the inferior longitudinal and was therefore not included [33].

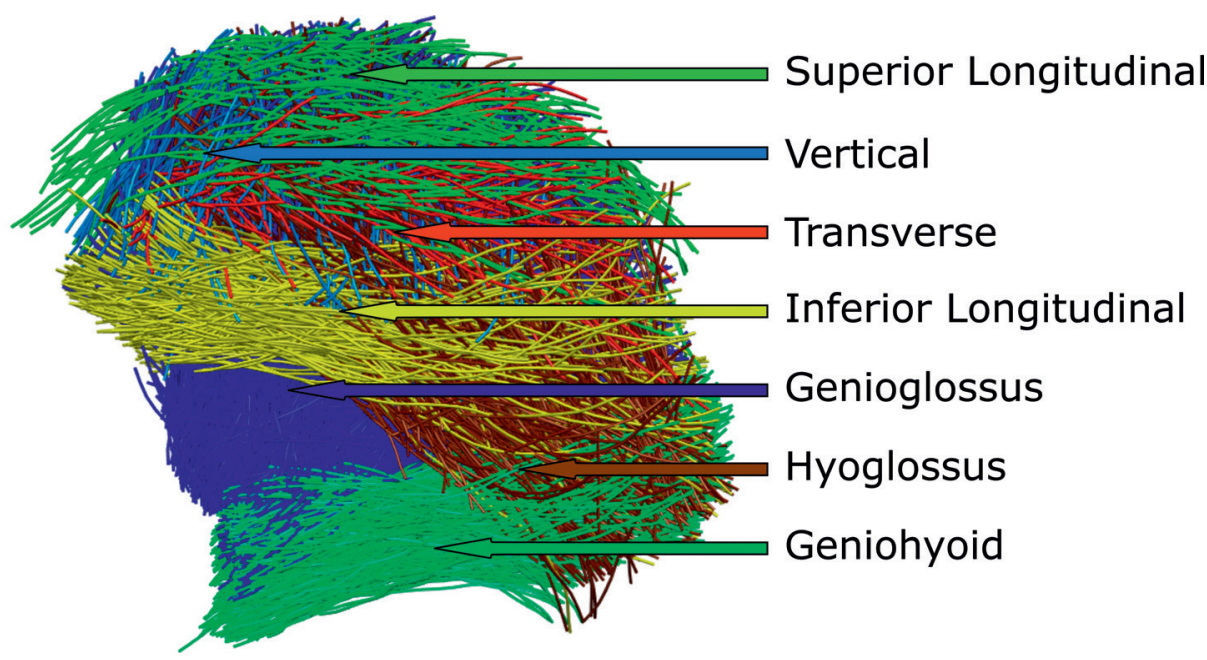

Figure 6.2 - Side view of the segmented fiber tracts of the atlas.

In Matlab R2019a (Mathworks, Natick, MA), these atlas tracks were mirrored in the midsagittal plane to ensure the symmetry of the atlas (Figure 6.1 A5). To remove faulty tracks, while preserving the muscle shape, the muscle tracts were filtered using the criteria shown in appendix I (Figure 6.1 A6). These criteria were chosen empirically based on the reduction in outliers and the expected curvature obtained from earlier anatomical research [9].

For each volunteer of the study group, the deformation fields obtained from the registration were used to morph the filtered tracts from the atlas into personalized tracts (Figure 6.1 P4). The personalized tracts may have been rotated during 
morphing and were therefore reoriented, based on the orientation of the muscles before morphing.

\section{FE model construction}

The tracks of both the atlas model and personalized models were converted into vector fields of muscle fiber direction (Figure 6.1 P5 and Figure 6.1 A7), using the following steps. For each muscle, a convex hull was calculated that enclosed the filtered tract (Figure 6.3 A-B). These convex hulls were filled with a uniformlydistributed grid of vectors, where the direction of these vectors was determined by an inverse distance interpolation of nearby tracks (Figure 6.3 C; Eq 6.1). This was done for both left and right muscles independently if applicable (Figure 6.3 D).

$$
\begin{gathered}
\boldsymbol{n}_{\text {grid }}(\boldsymbol{x})=\sum_{i}\left(1-\sqrt{\frac{d\left(\boldsymbol{x}, \text { track }_{i}\right)}{\max \left(d\left(\boldsymbol{x}, \text { track }_{i}\right)\right)}}\right) \boldsymbol{n}_{\text {track }_{i}} \\
\boldsymbol{n}_{\text {grid }}(\boldsymbol{x})=\frac{\boldsymbol{n}_{\text {grid }}(\boldsymbol{x})}{\left\|\boldsymbol{n}_{\text {grid }}(\boldsymbol{x})\right\|}
\end{gathered}
$$

Eq $6.1-\boldsymbol{n}_{\text {grid }}(\boldsymbol{x})$ the uniformly distributed grid of vectors within the convex hull and $\boldsymbol{n}_{\text {track }_{i}}$, the original tracks. $d\left(\boldsymbol{x}\right.$, track $\left._{i}\right)$ is the distance of vector $\boldsymbol{x}$ to track $_{i}$

The genioglossus muscle was divided into an oblique and horizontal part based on the estimated position of the short tendon [8]. With all tracks combined, a convex hull was generated to create a mesh of the tongue. In Meshlab [47], the HC Laplacian filter [48] was used to smoothen the mesh. Attachment points for the mandible and hyoid bones were determined based on the endpoints of the extrinsic muscle tracts from the atlas model.

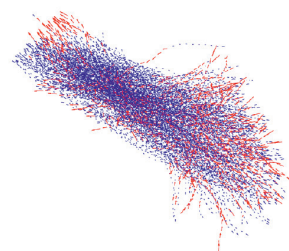

A

Figure 6.3 - The tracks (blue) and filtered tracks (red) from the inferior longitudinal muscle (a); the convex hull enclosing these tracks (b); a uniformly-distributed vector field based on the direction of the tracks within the convex hull (c); and vector fields of both left and right muscles (d)

Using ArtiSynth [49] - a platform for combined multibody and FE modeling - the muscle vector fields and tongue meshes of the atlas and the ten volunteers were integrated into a biomechanical model (Figure 6.1 P6, A8 and Figure 6.4) using the following steps, which are similar to those described by Kappert et al. [14]. The mesh of the tongue is embedded into an FE model consisting of cubic hexahedral 
elements. For every element, the direction of muscle contraction is determined by the previously calculated muscle vector fields. The attachment points to the hyoid and mandible were simulated by making FE nodes non-dynamic. For the mechanical properties of the muscle, a Mooney-Rivlin material was chosen with c10 equal to 1037 and c20 equal to 486. Rayleigh damping coefficients of $\alpha=40$ $\mathrm{s}^{-1}$ and $\beta=0.03$, and a density of $1040 \mathrm{~kg} / \mathrm{m}^{3}$ were used, comparable to those used by Buchaillard et al. [50], Stavness et al. [49] and Kappert et al. [14]. Muscle stressstrain functions were simulated using ArtiSynth's interpretation of the method by Blemker et al. [51] In this method, stress and strain are influenced by muscle activation based on the direction of that particular muscle.
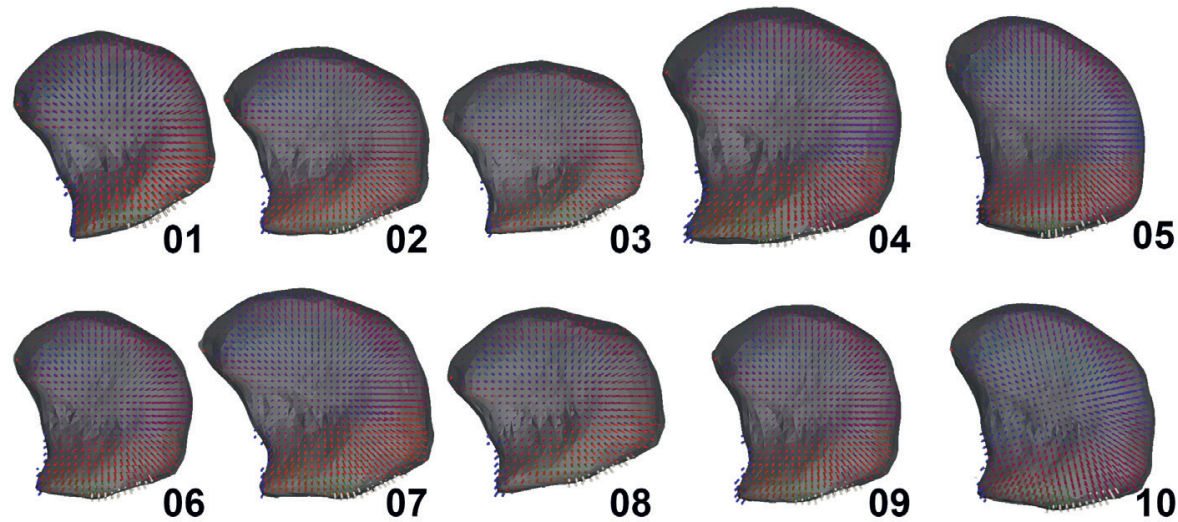

Figure 6.4 - Sagittal section view of personalized FE tongue models of the ten healthy volunteers. The direction of force of the muscle elements has been color-coded: anterior-posterior in red; right-left in green; and feet-head in blue. Bone attachment points are visualized as floating point outside the mesh. The mandible attachment points are visualized in blue, and those of the hyoid bone in white.

\section{Simulation and analysis}

Inverse simulation, provided by ArtiSynth [49,52], was used to instruct the tongue tip of the personalized FE models to consecutively move to a point anterior, inferior, left and right of its initial location. The predicted ROM was defined as the distance from the initial location to maximal deflection in one of the instructed directions.

Thetonguecanreach strainvalues of $200 \%$ for elongation and $160 \%$ for contraction [53]. Using the current hyperelastic model and mechanical properties, it was not possible to simulate these magnitudes of deformation. We, therefore, accepted that the magnitude of the predicted ROM would be less than the measured ROM. To be able to compare the differences between the predicted ROM's and their match to the measures ROM's, four scaling factors were calculated, one for every direction of movement. To make sure outliers would not affect the scaling factors, these factors were determined by an iterative process to achieve the maximum 
number of predicted ROMs $\left(\operatorname{ROM}_{\text {pred }}(i, j)\right)$ that were within the $\mathrm{Cl}\left(R O M_{2 \sigma}\right)$ of the measured ROM ( $\left.R O M_{\text {meas }}(i, j)\right)(\mathrm{Eq} 6.2)$.

$$
\begin{aligned}
& i=1, \cdots, 4 \quad \text { Index for the } 4 \text { different movements } \\
& j=1, \cdots, 10 \text { Index for the } 10 \text { volunteers from the study group } \\
& R O M_{\text {pred }}(i, j) \text { Predicted ROM } \\
& R O M_{\text {meas }}(i, j) \text { Measured ROM } \\
& S_{i} \quad \text { The scaling factor applied to predicted } R O M_{\text {pred }}(i, j) \\
& R O M_{2 \sigma} \text { Twice the standard deviation of the measured ROM (i.e. } 6 \mathrm{~mm} \text { ) } \\
& S_{i}=\underset{S_{i}}{\arg \max } \sum_{j=1}^{10} \llbracket\left|S_{i} \cdot R O M_{\text {pred }}(i, j)-R O M_{\text {meas }}(i, j)\right|<R O M_{2 \sigma} \rrbracket \text { where } \llbracket x \rrbracket \\
& =\left\{\begin{array}{l}
0 \text { if } x=\text { false } \\
1 \text { if } x=\text { true }
\end{array}\right.
\end{aligned}
$$

Eq 6.2 - Through an iterative process, the scaling factor should result in the highest number of predictions $R O M_{\text {pred }}(i, j)$ within the bounds $R O M_{2 \sigma}$ of the measured $\operatorname{ROM}\left(\operatorname{ROM}_{\text {meas }}(i, j)\right)$

The predicted ROM was compared to the in vivo measured ROM of the individual on whom the personalized model was based. To show the benefit of personalization, also the Atlas model (essentially a generic model) will be compared with the measured ROM. Only when the personalized models perform better than the atlas, we can conclude that personalization improves the ROM prediction.

Previously, the precision of the ROM measurements (quantified by the standard deviation) was determined to range from $2.3 \mathrm{~mm}$ to $3.2 \mathrm{~mm}$ [35]. We, therefore, assumed a precision of $3 \mathrm{~mm}$ ( $3.2 \mathrm{~mm}$ rounded off) for all ROM measurements. If a predicted ROM fell within $95 \%$ confidence interval $(\mathrm{Cl})$, i.e. within two times the standard deviation, we judged the measurement to be correct.

\subsection{RESULTS}

Visually, the FOD maps were well aligned to the atlas. The mean $\mathrm{L}_{2}$-norm between the FOD maps and the atlas was 0.302 (SD 0.030). The average angular correlation coefficient was 0.634 (SD 0.057). 

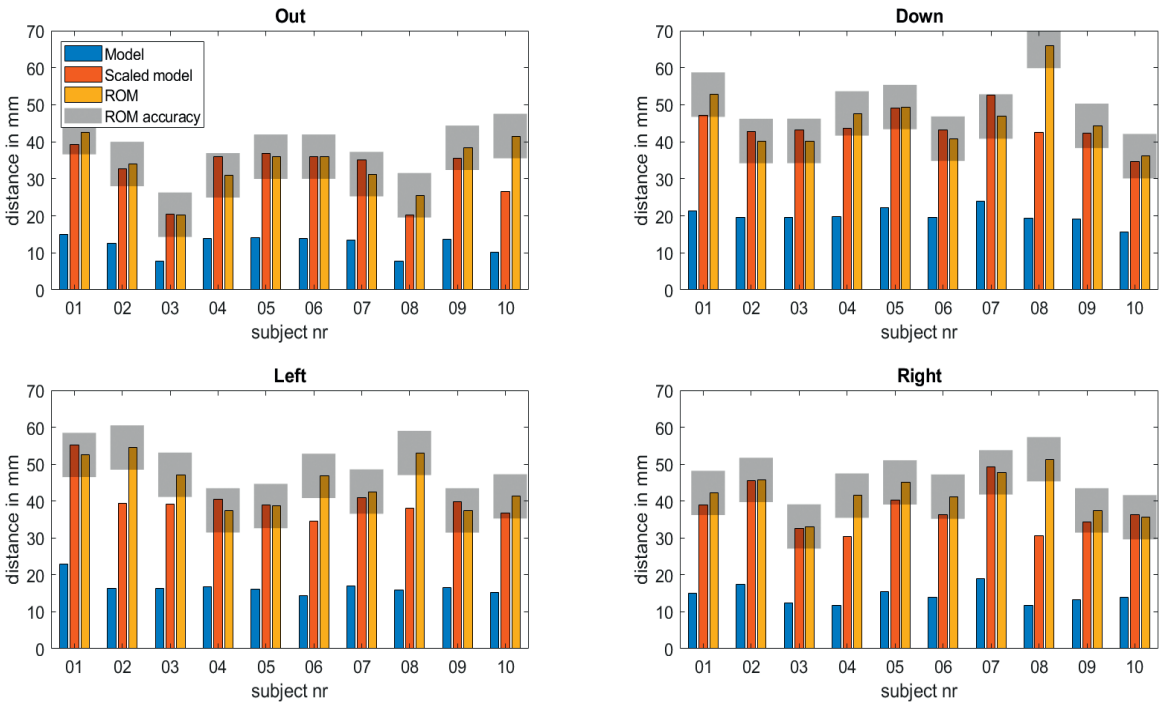

Figure 6.5 - Range-of-motion (ROM) in $\mathrm{mm}$ for the ten healthy volunteers (01-10) and the Atlas (Generic model), for protrusion, and the down, left, and right movements. The predicted ROM of the personalized and atlas (generic model) is given in blue, the scaled predicted ROM in orange, and the measured ROM in yellow. The grey box depicts the interval of two times the standard deviation of the measured ROM within which the predicted ROM values of both atlas and personalized models are assumed to be accurate

In Figure 6.5, the distances for specific tongue movements of both the measured ROM and predicted ROM are shown for all ten subjects. For the predicted ROM, both the scaled and non-scaled movements are shown. The scaling factors are 2.6 for protrusion, 2.2 for down, 2.4 for left, and 2.6 for right. Protrusion and down movements show the best agreement between predictions and measurements, as nine out of ten (90\%) predicted ROM's are within the $\mathrm{Cl}$. Eight movements (80\%) to the right were predicted to be within the $\mathrm{Cl}$, but for the movement to the left, only six (60\%) were predicted to be within the interval. In total, 32 out of the 40 predictions ( 4 movements, 10 volunteers) from the personalized models were within the $\mathrm{Cl}$. The largest disagreements between the prediction and in vivo measurement were found in subject 08. 


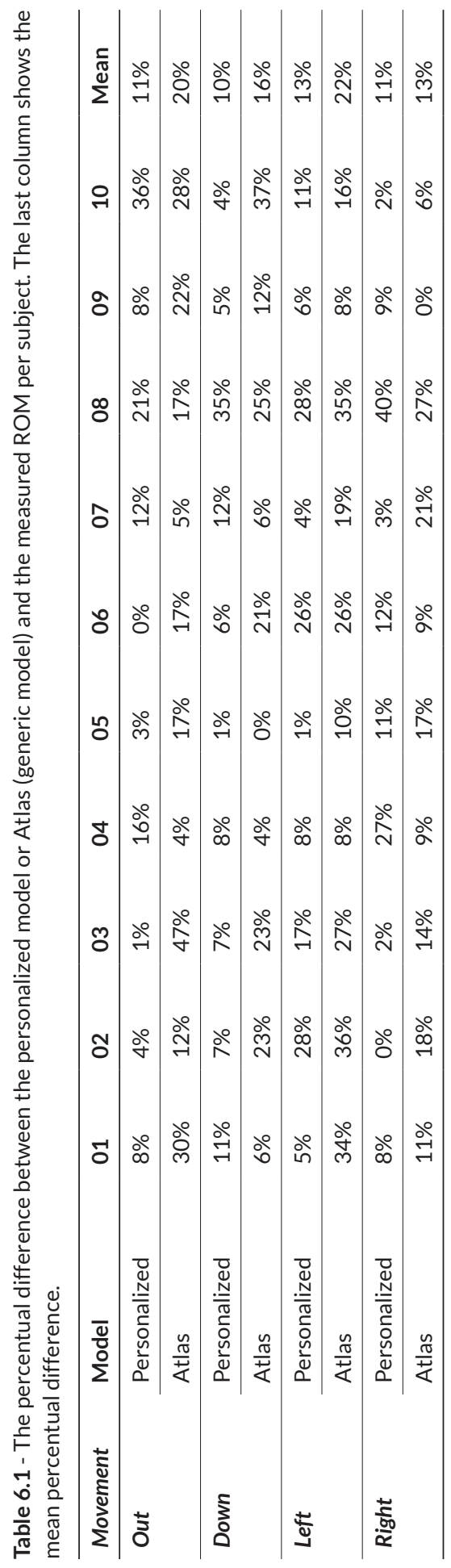


In Table 6.1 the percentual differences of the models with the measured ROM are shown for every subject. The model that predicts the ROM better differs between subject and movement, but a majority of the measurements are predicted better using the personalized model. The mean percentual difference per movement shows that for all movements the difference with the measured ROM is lower for the personalized models.

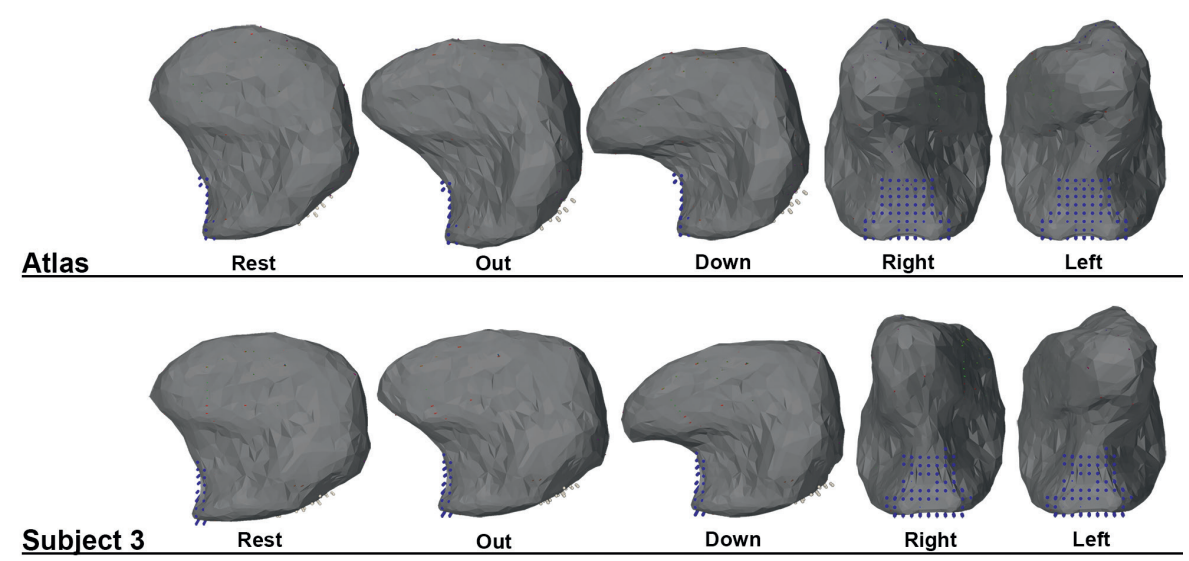

Figure 6.6 - An example of the maximum range in the ROM prediction for protrusion, down, left, and right using the personalized model of subject 1,10 and the atlas.

In Figure 6.6 the atlas model and the personalized model of subject 3 are shown within the Artisynth environment. For the four simulated movements, the maximal extension is shown. Subject 3 demonstrated a ROM that that in 3 out of 4 movements could not be predicted using the atlas, but could be using the personalized model. The movement of the atlas, relative to its rest-state, looks larger in most directions than the personalized model as can be confirmed by the bar charts in Figure 6.5. Also, the tongue moves more upwards during the right movement. The magnitude of the movements of both models is smaller than what would be expected from a real tongue.

\subsection{DISCUSSION}

This study was a first approach to combine CSD MRI and FE modeling to create personalized biomechanical models of ten healthy volunteers. For the inverse simulations of protrusion and down movements, $90 \%$ of the predicted movements were accurate; for the right movements, $80 \%$ were predicted correctly; and for the left movements, only $60 \%$ were predicted correctly. Overall, the personalized models predicted $80 \%$ of the movements correctly, compared to only $50 \%$ for the atlas. Therefore, the personalized models predicted the ROM better than an atlas or a single generic model could. 
Although the downward movement of subject 8 with a relatively small tongue was exceptionally high, this measurement was confirmed to be correct upon reviewing the images from the 3D camera. The model was not able to reproduce this large ROM, which may indicate that a large ROM is not only a result of tongue muscle morphology. The ROM may also have been affected by other quantities that were not accounted for, such as the number of motor units or the stiffness, anisotropy, and density of the tissue.

In the posterior part or base of the tongue, breathing motion impaired the tracking of the superior longitudinal and transverse muscle [33]. Although these muscle tracts were filtered less vigorously, this could not resolve the absence of muscle tracts. Fortunately, the effect of the absence of these tracks is expected to be minimal, as the simulation of the ROM is less dependent on the musculature of the posterior tongue. Other artifacts, such as those caused by ferromagnetic crowns, resulted in signal voids in the diffusion-weighted images, and therefore gaps in the tractography of the tongue. As the biomechanical models were based on the atlas, where such gaps are not present, we assume these artifacts that occurred in individual data sets would cause minimal errors in the personalized models.

The genioglossus and geniohyoid muscles form one large continuous fan of tracks. As described in the literature some FE models divide the genioglossus into an anterior, middle, and posterior part [15,54-56], while others separate the genioglossus into a horizontal and an oblique subdivision $[8,10,57]$. We chose the latter because the location of the short tendon could be inferred from our atlas and could, therefore, be used as an anatomical marker to split the genioglossus in two. As the styloglossus could not be distinguished from the inferior longitudinal muscle in the fiber tracking, the styloglossus was omitted from the model. The effect on our simulations was expected to be limited since the styloglossus is mainly involved in retracting the tongue and swallowing.

Similar to CSD in the brain, the apparent fiber density could be derived from CSD in muscles, which should in principle relate to muscle strength [58,59]. Therefore, incorporating this apparent fiber density into our biomechanical models might improve the ROM predictions. However, since CSD MRI in the tongue is subject to higher noise levels and more motion artifacts than for example in the brain, in our opinion, the apparent fiber density can currently not be quantified accurately. We, therefore, assumed that the vectors describing the muscle direction where equally distributed within the area of specific muscles.

While large parts of the methods were automated, some key elements were still done manually. For the atlas, the segmentation of the fiber tracts and 
the subsequent filtering were done manually. Techniques to automate these segmentation steps are not matured yet and therefore manual input is still needed. For the personalized models, only the initial masks were manually delineated. After this step, the models could be processed without manual interference.

In this study, we mirrored the atlas to make it symmetric. However, by applying the displacement fields to create personalized biomechanical models, asymmetry was reintroduced. In this study, the orientation of the personalized biomechanical models was based on the former position of the tracks within the atlas. This leads to small lateral asymmetry in the distribution of muscles. An alternative method would be to label based on its new midline. However, determining the exact midline remains challenging, and without a gold standard, there is no way to determine which method is best.

Similar to previous work [14], we used hexahedral cubic elements with embedded muscles and mesh for the FE model, which do not optimally represent the shape of a surface. As stated in the previous work the effect of this method on the mobility of the model is minimal. The choice for this embedded design was made so that in the future the virtual surgery method introduced in the aforementioned study can be used in combination with the personalization proposed in the current study.

In this study, optical tracking of the tongue tip was used to determine the ROM. Based on the results of Kappertet al. [35] that proofed the up movement unreliable, it was decided not to use this movement in the comparison. Not only the tongue but also the mandible and hyoid bone assist the tongue tip in reaching the desired position. How much influence these structures have on the tongue ROM depends on the anatomy, innervation, and brain-muscle control. This influence had not been measured and, instead, a marker on the mandible was used to compensate for the movement of the mandible [35]. This marker may however not always reliably compensate for all complex movements, and an error should be expected in the measured ROM. Predicted ROMs were therefore judged on whether they fell within the $\mathrm{Cl}$ of this error. This $\mathrm{Cl}$ was relatively wide, and might, therefore, have hampered the correct judgment of small variations between the predicted ROM and measured ROM.

In the biomechanical models, the magnitude of the predicted ROM was much smaller than that of the measured ROM which, therefore, had to be scaled. Incorporating movement of adjacent connected structured such as the hyoid bone might improve the range. Also, the mechanical properties were based on the model of Buchaillard et al. [50], which uses stiffer material properties than those originally measured in a cadaver study, to simulate an active state of the tongue [60]. Moreover, hyperelastic material models used in most FE tongue 
models cannot cover all the complex properties of the tongue [15]. In this study, the FE model became unstable in extreme positions using lower stiffness values. Because the same stiffness is used for all personalized models, we assumed that the relative difference between models would still be comparable, independent of the stiffness value used. However, stiffness was not the only limiting factor. Also, the specific muscle morphology obtained from CSD MRI contributed to the small magnitude of motion that is smaller than other non-personalized models in the literature that use the same mechanical properties [14,15,50]. Manual editing of the muscle morphology might improve the magnitude of motion, but it was not considered as it would compromise the goal of this research, which was to automate FE modeling based on CSD data. Finally, the scaling of the predicted ROMs was, although very close, not the same in every movement direction. This corresponded to an asymmetry in the measured ROM, specifically, a deviation to the left. As explained previously [35], this may have been caused by the order of instructions given by the investigator.

In conclusion, we demonstrated that biomechanical models based on CSD MRI predict tongue ROM better than an atlas or generic model. To our knowledge, we are the first to report this personalization step for improving the prediction of tongue mobility. In the future, personalization may improve other biomechanical models such as those of speech and swallowing, potentially leading to better simulations of actual tongue functionality. In rehabilitation after tongue cancer surgery, models can potentially be used to simulate the tongue function that could be regained by practice. In the preoperative setting, we would expect an even larger potential for the prediction of tongue function, as alterations in tongue shape and musculature due to tumor growth would also be accounted for. Therefore, these results harbor a promising perspective for the development of biomechanical models that would better predict function loss of oral cancer patients and thus improve the choice of treatment in these patients. 


\subsection{REFERENCES}

1. UK Cancer Research. Head and neck cancers incidence statistics [Internet]. 2019 [cited 26 Apr 2019]. Available: https://www.cancerresearchuk.org/healthprofessional/cancer-statistics/statistics-by-cancer-type/head-and-neck-cancers/ incidence\#heading-Four

2. Tota JE, Anderson WF, Coffey C, Califano J, Cozen W, Ferris RL, et al. Rising incidence of oral tongue cancer among white men and women in the United States, 1973-2012. Oral Oncol. 2017;67: 146-152. doi:10.1016/j.oraloncology.2017.02.019

3. Konstantinović VS, Dimić ND. Articulatory function and tongue mobility after surgery followed by radiotherapy for tongue and floor of the mouth cancer patients. Br J Plast Surg. 1998;51: 589-593. doi:10.1054/bjps.1998.0202

4. Kreeft AM, Van Der Molen L, Hilgers FJ, Balm AJ. Speech and swallowing after surgical treatment of advanced oral and oropharyngeal carcinoma: A systematic review of the literature. Eur Arch Oto-Rhino-Laryngology. Springer; 2009;266: 1687-1698. doi:10.1007/s00405-009-1089-2

5. Kreeft AM, Tan IB, van den Brekel MWM, Hilgers FJ, Balm AJM. The surgical dilemma of 'functional inoperability' in oral and oropharyngeal cancer: current consensus on operability with regard to functional results. Clin Otolaryngol. 2009;34: 140-146. doi:10.1111/j.1749-4486.2009.01884.x

6. Bressmann T, Sader R, Whitehill TL, Samman N. Consonant intelligibility and tongue motility in patients with partial glossectomy. J Oral Maxillofac Surg. 2004;62: 298-303. doi:10.1016/j.joms.2003.04.017

7. Matsui Y, Ohno K, Yamashita Y, Takahashi K. Factors influencing postoperative speech function of tongue cancer patients following reconstruction with fasciocutaneous/ myocutaneous flaps-a multicenter study. Int J Oral Maxillofac Surg. 2007;36: 601609. doi:10.1016/j.ijom.2007.01.014

8. Sanders I, Mu L. A three-dimensional atlas of human tongue muscles. Anat Rec. 2013;296: 1102-1114. doi:10.1002/ar.22711

9. Takemoto H. Morphological Analyses of the Human Tongue Musculature for ThreeDimensional Modeling. J Speech, Lang Hear Res. 2001;44: 95-107. doi:10.1044/10924388(2001/009)

10. Mu L, Sanders I. Human tongue neuroanatomy: Nerve supply and motor endplates. Clin Anat. 2010;23: 777-791. doi:10.1002/ca.21011

11. Slaughter $\mathrm{K}, \mathrm{Li} \mathrm{H}$, Sokoloff AJ. Neuromuscular Organization of the Superior Longitudinalis Muscle in the Human Tongue. Cells Tissues Organs. 2005;181: 51-64. doi:DOI/10.1159/000089968

12. Van Alphen MJAJA, Eskes M, Smeele LEE, Balm AJMJM, van der Heijden F. In vivo intraoperative hypoglossal nerve stimulation for quantitative tongue motion analysis. Comput Methods Biomech Biomed Eng Imaging Vis. Taylor \& Francis; 2017;5: 409415. doi:10.1080/21681163.2015.1072056

13. Stone M, Woo J, Lee J, Poole T, Seagraves A, Chung M, et al. Structure and variability in human tongue muscle anatomy. Comput Methods Biomech Biomed Eng Imaging Vis. 2018;6: 499-507. doi:10.1080/21681163.2016.1162752 
14. Kappert KDR, van Alphen MJA, van Dijk S, Smeele LE, Balm AJM, van der Heijden F. An interactive surgical simulation tool to assess the consequences of a partial glossectomy on a biomechanical model of the tongue. Comput Methods Biomech Biomed Engin. 2019;22: 827-839. doi:10.1080/10255842.2019.1599362

15. Hermant N, Perrier P, Payan Y. Human Tongue Biomechanical Modeling. In: Payan Y, Ohayon JBT-B of LO, editors. Biomechanics of Living Organs: Hyperelastic Constitutive Laws for Finite Element Modeling. Oxford: Academic Press; 2017. pp. 395-411. doi:10.1016/B978-0-12-804009-6.00019-5

16. Buchaillard S, Brix M, Perrier P, Payan Y. Simulations of the consequences of tongue surgery on tongue mobility: Implications for speech production in post-surgery conditions. Int J Med Robot Comput Assist Surg. 2007;3: 252-261. doi:10.1002/ rcs.142

17. Couteau B, Payan Y, Lavallée S. The mesh-matching algorithm: an automatic 3D mesh generator for finite element structures. J Biomech. 2000;33: 1005-1009. doi:10.1016/ S0021-9290(00)00055-5

18. Sigal IA, Hardisty MR, Whyne CM. Mesh-morphing algorithms for specimenspecific finite element modeling. J Biomech. 2008;41: 1381-1389. doi:10.1016/j. jbiomech.2008.02.019

19. Fernandez JW, Mithraratne P, Thrupp SF, Tawhai MH, Hunter PJ. Anatomically based geometric modelling of the musculo-skeletal system and other organs. Biomech Model Mechanobiol. 2004;2: 139-155. doi:10.1007/s10237-003-0036-1

20. Bucki M, Lobos C, Payan Y. A fast and robust patient specific Finite Element mesh registration technique: Application to 60 clinical cases. Med Image Anal. Elsevier B.V.; 2010;14: 303-317. doi:10.1016/j.media.2010.02.003

21. Grassi L, Hraiech N, Schileo E, Ansaloni M, Rochette M, Viceconti M. Evaluation of the generality and accuracy of a new mesh morphing procedure for the human femur. Med Eng Phys. 2011;33: 112-120. doi:10.1016/j.medengphy.2010.09.014

22. Bijar A, Rohan P-Y, Perrier P, Payan Y. Atlas-Based Automatic Generation of SubjectSpecific Finite Element Tongue Meshes. Ann Biomed Eng. 2016;44: 16-34. doi:10.1007/ s10439-015-1497-y

23. Barber DC, Oubel E, Frangi AF, Hose DR. Efficient computational fluid dynamics mesh generation by image registration. Med Image Anal. 2007;11: 648-662. doi:10.1016/j. media.2007.06.011

24. Nesme M, Kry PG, Jeřábková L, Faure F. Preserving topology and elasticity for embedded deformable models. ACM Trans Graph. 2009;28: 1-9. doi:10.1145/1531326.1531358

25. Sánchez CA, Li Z, Hannam AG, Abolmaesumi P, Agur A, Fels S. Constructing Detailed Subject-Specific Models of the Human Masseter. Lecture Notes in Computer Science. Springer; 2017. pp. 52-60. doi:10.1007/978-3-319-67552-7_7

26. Van Donkelaar CC, Kretzers LJG, Bovendeerd PHM, Lataster LMA, Nicolay K, Janssen JD, et al. Diffusion tensor imaging in biomechanical studies of skeletal muscle function. J Anat. 1999;194: 79-88. doi:10.1017/S0021878298004397

27. Basser PJ, Pajevic S, Pierpaoli C, Duda J, Aldroubi A. In vivo fiber tractography using DT-MRI data. Magn Reson Med. 2000;44: 625-632. doi:10.1002/15222594(200010)44:4<625::AID-MRM17>3.0.CO;2-O

28. Shinagawa H, Murano EZ, Zhuo J, Gullapalli RP, Landman B, Prince JL, et al. Tongue muscle fiber tracking during tongue protrusion and rest. J Acoust Soc Am. 2006;120: 3354-3354. doi:10.1121/1.4781440 
29. Ye C, Murano E, Stone M, Prince JL. A Bayesian approach to distinguishing interdigitated tongue muscles from limited diffusion magnetic resonance imaging. Comput Med Imaging Graph. 2015;45: 63-74. doi:10.1016/j.compmedimag.2015.07.005

30. Napadow VJ, Chen Q, Mai V, So PTC, Gilbert RJ. Quantitative analysis of threedimensional-resolved fiber architecture in heterogeneous skeletal muscle tissue using NMR and optical imaging methods. Biophys J. 2001;80: 2968-2975. doi:10.1016/ S0006-3495(01)76262-5

31. Heemskerk AM, Sinha TK, Wilson KJ, Ding Z, Damon BM. Repeatability of DTIbased skeletal muscle fiber tracking. NMR Biomed. 2010;23: 294-303. doi:10.1002/ nbm.1463

32. Mijailovich SM, Stojanovic B, Kojic M, Liang A, Wedeen VJ, Gilbert RJ. Derivation of a finite-element model of lingual deformation during swallowing from the mechanics of mesoscale myofiber tracts obtained by MRI. J Appl Physiol. 2010/08/05. American Physiological Society; 2010;109: 1500-1514. doi:10.1152/japplphysiol.00493.2010

33. Voskuilen L, Mazzoli V, Oudeman J, Balm AJM, van der Heijden F, Froeling M, et al. Crossing muscle fibers of the human tongue resolved in vivo using constrained spherical deconvolution. J Magn Reson Imaging. John Wiley \& Sons, Ltd; 2019;50: 96-105. doi:10.1002/jmri.26609

34. Voskuilen L, Smeele LE, Balm AJM, Heijden F Van Der, Strijkers GJ, Nederveen AJ. Generation of a muscle fibre orientation atlas of the in vivo tongue. Proc IntI Soc Mag Reson Med. Paris; 2018. p. 26:5217.

35. Kappert KDR, van Alphen MJA, Smeele LE, Balm AJM, van der Heijden F. Quantification of tongue mobility impairment using optical tracking in patients after receiving primary surgery or chemoradiation. Kimple RJ, editor. PLoS One. 2019;14: e0221593. doi:10.1371/journal.pone.0221593

36. Veraart J, Novikov DS, Christiaens D, Ades-aron B, Sijbers J, Fieremans E. Denoising of diffusion MRI using random matrix theory. Neuroimage. Elsevier Inc.; 2016;142: 1-28. doi:10.1016/j.neuroimage.2016.08.016

37. Smith SM, Jenkinson M, Woolrich MW, Beckmann CF, Behrens TEJ, Johansen-Berg H, et al. Advances in functional and structural MR image analysis and implementation as FSL. Neuroimage. 2004;23: 208-219. doi:10.1016/j.neuroimage.2004.07.051

38. Andersson JLR, Sotiropoulos SN. An integrated approach to correction for offresonance effects and subject movement in diffusion MR imaging. Neuroimage. The Authors; 2016;125: 1063-1078. doi:10.1016/j.neuroimage.2015.10.019

39. Yushkevich PA, Piven J, Hazlett HC, Smith RG, Ho S, Gee JC, et al. User-guided 3D active contour segmentation of anatomical structures: significantly improved efficiency and reliability. Neuroimage. 2006;31: 1116-28. doi:10.1016/j.neuroimage.2006.01.015

40. Tournier JD, Calamante F, Connelly A. MRtrix: Diffusion tractography in crossing fiber regions. Int J Imaging Syst Technol. 2012;22: 53-66. doi:10.1002/ima.22005

41. Tournier JD, Calamante F, Connelly A. Determination of the appropriate $b$ value and number of gradient directions for high-angular-resolution diffusion-weighted imaging. NMR Biomed. 2013;26: 1775-1786. doi:10.1002/nbm.3017

42. Tournier J-D, Calamante F, Connelly A. Robust determination of the fibre orientation distribution in diffusion MRI: non-negativity constrained super-resolved spherical deconvolution. Neuroimage. 2007;35: 1459-1472. doi:10.1016/j. neuroimage.2007.02.016 
43. Raffelt D, Tournier JD, Fripp J, Crozier S, Connelly A, Salvado O. Symmetric diffeomorphic registration of fibre orientation distributions. Neuroimage. Elsevier B.V.; 2011;56: 1171-1180. doi:10.1016/j.neuroimage.2011.02.014

44. Raffelt D, Tournier JD, Crozier S, Connelly A, Salvado O. Reorientation of fiber orientation distributions using apodized point spread functions. Magn Reson Med. 2012;67: 844-855. doi:10.1002/mrm.23058

45. Tournier J-D, Calamante F, Connelly A. Improved probabilistic streamlines tractography by 2 nd order integration over fibre orientation distributions. Intl Soc Mag Reson Med. 2010. p. 18:1670.

46. Wang R, Benner T. Diffusion toolkit: a software package for diffusion imaging data processing and tractography. Proc Intl Soc Mag Reson Med. 2007.

47. Cignoni P, Cignoni P, Callieri M, Callieri M, Corsini M, Corsini M, et al. MeshLab: an Open-Source Mesh Processing Tool. Sixth Eurographics Ital Chapter Conf. 2008; 129136. doi:10.2312/LocalChapterEvents/ItalChap/ItalianChapConf2008/129-136

48. Vollmer J, Mencl R, Muller H. Improved Laplacian Smoothing of Noisy Surface Meshes. Comput Graph Forum. 1999;18: 131-138. doi:10.1111/1467-8659.00334

49. Lloyd JE, Stavness I, Fels S. ArtiSynth: A Fast Interactive Biomechanical Modeling Toolkit Combining Multibody and Finite Element Simulation. Studies in Mechanobiology, Tissue Engineering and Biomaterials. 2012.pp. 355-394. doi:10.1007/8415_2012_126

50. Buchaillard S, Perrier P, Payan Y. A biomechanical model of cardinal vowel production: muscle activations and the impact of gravity on tongue positioning. J Acoust Soc Am. 2009;126: 2033-2051. doi:10.1121/1.3204306

51. Blemker SS, Pinsky PM, Delp SL. A 3D model of muscle reveals the causes of nonuniform strains in the biceps brachii. J Biomech. 2005;38: 657-665. doi:10.1016/j. jbiomech.2004.04.009

52. Stavness I, Lloyd JE, Fels S. Automatic prediction of tongue muscle activations using a finite element model. J Biomech. Elsevier; 2012;45: 2841-2848. doi:10.1016/j. jbiomech.2012.08.031

53. Napadow VJ, Chen Q, Wedeen VJ, Gilbert RJ. Intramural mechanics of the human tongue in association with physiological deformations. J Biomech. 1999;32: 1-12. doi:10.1016/S0021-9290(98)00109-2

54. Harandi NM, Woo J, Stone M, Abugharbieh R, Fels S. Subject-specific biomechanical modelling of the tongue: Analysis of muscle activations during speech. Proceedings of the 10th International Seminar on Speech Production, ISSP 2014. 2014. pp. 174-177.

55. Wu X, Dang J, Stavness I. Iterative method to estimate muscle activation with a physiological articulatory model. Acoust Sci Technol. 2014;35: 201-212. doi:10.1250/ ast.35.201

56. Dabbaghchian S, Arnela M, Engwall O, Guasch O, Stavness I, Badin P, et al. Using a Biomechanical Model and Articulatory Data for the Numerical Production of Vowels. INTERSPEECH. 2016;m: 3569-3573. doi:10.21437/Interspeech.2016-1500

57. Honda K, Murano EZ, Takano S, Masaki S, Dang J. Anatomical considerations on the extrinsic tongue muscles for articulatory modeling. Proceedings of Meetings on Acoustics. 2013. pp. 60-270. doi:10.1121/1.4800262

58. Raffelt D, Tournier JD, Rose S, Ridgway GR, Henderson R, Crozier S, et al. Apparent Fibre Density: A novel measure for the analysis of diffusion-weighted magnetic resonance images. Neuroimage. Elsevier Inc.; 2012;59: 3976-3994. doi:10.1016/j. neuroimage.2011.10.045 
59. Miller JL, Watkin KL, Chen MF. Muscle, Adipose, and Connective Tissue Variations in Intrinsic Musculature of the Adult Human Tongue. J Speech, Lang Hear Res. 2002;45: 51-65. doi:10.1044/1092-4388(2002/004)

60. Gerard JMM, Ohayon J, Luboz V, Perrier P, Payan Y. Non-linear elastic properties of the lingual and facial tissues assessed by indentation technique: Application to the biomechanics of speech production. Med Eng Phys. Elsevier; 2005;27: 884-892. doi:10.1016/j.medengphy.2005.08.001 


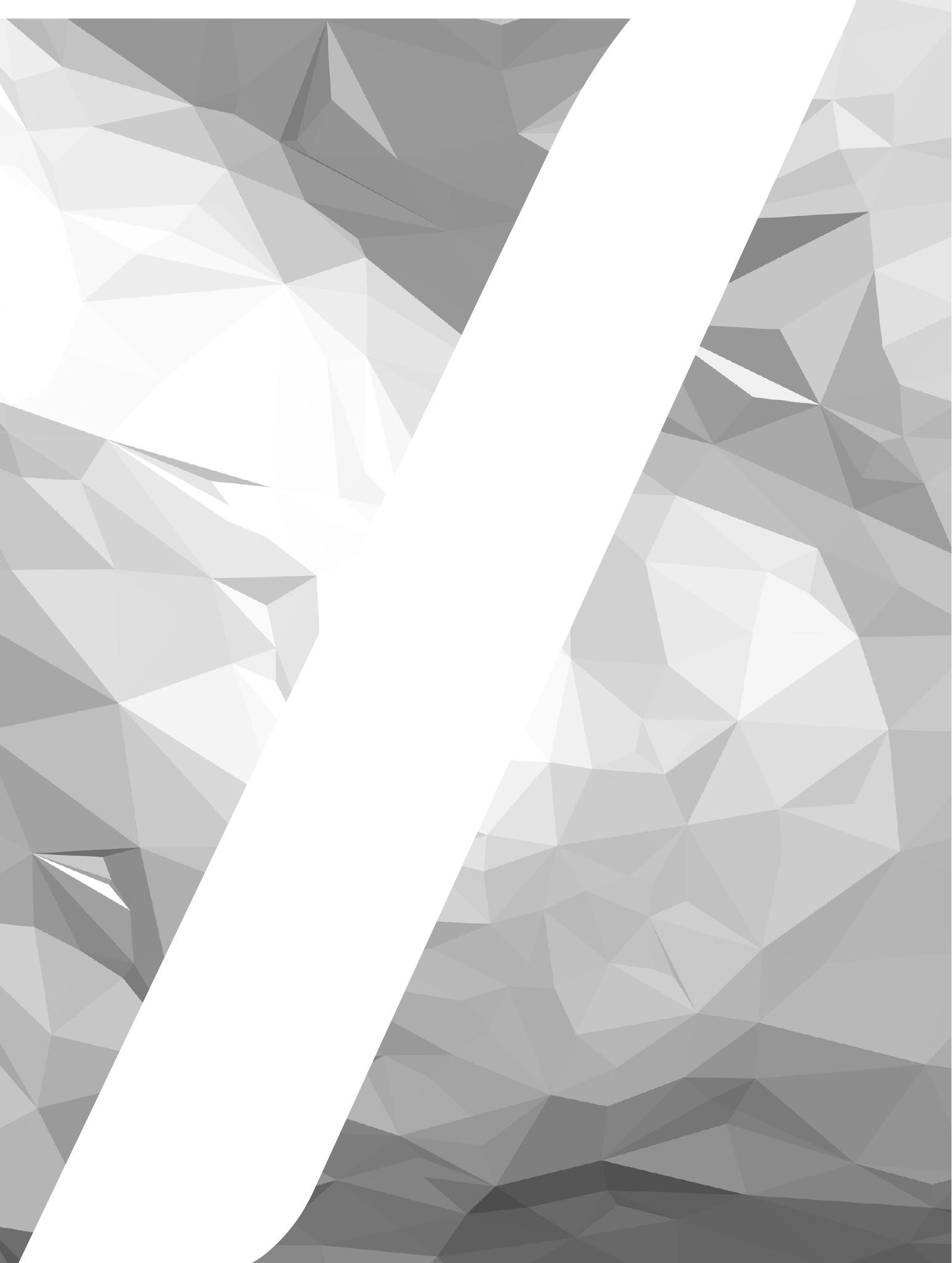




\title{
PREDICTION OF TONGUE
}

FUNCTION AFTER A PARTIAL

GLOSSECTOMY USING

PERSONALIZED CSD MRI

DRIVEN BIOMECHANICAL

MODELS: A PILOT STUDY

\author{
K.D.R. Kappert \\ L. Voskuilen \\ A.F. de Geer \\ M.J.A. van Alphen \\ B. Jasperse \\ A.J. Nederveen \\ L.E. Smeele \\ A.J.M. Balm \\ F. van der Heijden
}

This chapter is not yet submitted

This work was presented at the Technical Innovations In Medicine congress, Webinar (2020) 


\section{ABSTRACT}

Treatments of head and neck cancers, particularly of tongue cancer, are notorious for their destructive effect on function and therefore quality of life. Post-treatment functional consequences are difficult to predict statistically, but biomechanical models could be a viable alternative in simulating the complex muscle structure, innervation, and tissue mechanics of the tongue. Previously, we developed a tool to simulate a surgical intervention using a biomechanical model of the tongue. It showed realistic deformations after partial glossectomy and primary closure. Another recent development is the use of diffusion-weighted MRI to reconstruct the crossing muscle fiber architecture using Constrained Spherical Deconvolution (CSD), which allows for multiple muscle directions to be divined in one spot. This study aims to combine the aforementioned techniques to create personalized post-operative biomechanical models and to compare these with a generic, non-personalized, models.

Eight tongue cancer patients were included in this study. All patients underwent a pre-operative diffusion-weighted scan. Following excision, a micro-CT of the specimen was made to provide $3 \mathrm{D}$ dimensions for the simulation of the surgical wounds. Two observers performed a surgical simulation using both a personalized model (based on a specific patient) and a generic model. To assess intra- and inter-observer variability, the procedure was repeated independently by each observer multiple times. The predictive capacity of the models was tested using pre- and post-operative standardized Range Of Motion (ROM) measurements of the tongue in-vivo. Pre-operative in-vivo ROMs were similar to those of healthy participants from a previous study, and post-operative in-vivo ROMs also matched the post-operative measurements of a previous study.

Compared with the generic model, the personalized model showed larger postoperative impairments, which were closer to the in-vivo ROM than the effects in the generic model. In terms of the number of correct predictions, however, the full potential of this model could not be demonstrated due to unexpected MRI imaging failures in 8 out of ten patients. BO inhomogeneities and involuntary movements were likely the causes behind these artifacts. Nevertheless, this research demonstrated a unique approach in the prediction of post-operative tongue function. Future studies, using improved methods to acquire CSD images, can utilize this method to create truly personalized models. 


\section{$7.1 \quad$ INTRODUCTION}

With approximately 250,000 cases annually, head and neck cancer accounts for $4 \%$ of all malignancies in Europe [1]. The tongue and the base of the tongue are both essential for speech, swallowing, and mastication, and these regions account for around $20 \%$ of all head and neck carcinomas [2,3]. While not the most common cancer, head and neck cancers are notorious for their destructive effect on function and tend to have a significant negative impact on Quality of Life (QoL) [4-9].

Lower-staged tongue carcinomas (stage T1 to T3) are usually treated surgically [9-11]. In advanced disease or in cases of anatomical or functional inoperability, organ-sparing treatment is preferred, such as radiation or chemoradiation therapy $[12,13]$. However, if severe functional consequences are expected in lower-staged tumors, organ sparing treatment can be an option as well [4,14].

Post-treatment functional consequences are difficult to predict. Due to the complex anatomy and innervation of the upper aerodigestive tract generally, and the tongue more specifically, the problem is simply too complicated to allow for prediction without an objective tool $[15,16]$. In this context, biomechanical models, which can simulate the complex muscle structure, innervation, and tissue mechanics, are expected to be a suitable candidate [17].

In the past 25 years, progress has been made in the biomechanical modeling of the tongue, with many models being developed to study speech production and speech motor control [18-23]. In 2006, Gerard et al. [24] elaborated on the work of Wilhelms-Tricarico [25] by creating a Finite Element (FE) model of the tongue, based on data of the Visible Human Project [26] and MRI data of a single participant [20]. Using this model, Buchaillard et al. [23] simulated the postsurgical mobility by stiffening parts of the tongue. Furthermore, Fujita et al. [27] simulated a free-flap surgical approach by altering both the shape and stiffness of a model. However, these models were not able to simulate primary closure of the wound. In 2019, Kappert et al. [17] used the model from Buchaillard et al. [21] as a basis to create a user-friendly tool to simulate surgery. With this tool, sutures could be used as primary closure, thus simulating realistic deformation of tissue.

The above-mentioned proof-of-concepts showed promising results for the use of biomechanical models in the prediction of post-operative tongue mobility and possible changes in speech and swallowing function. The aforementioned models were mainly based on one participant, and could therefore not be translated to other patients. This is why, in another study, we tried to personalize the tongue models by reconstructing muscle bundles with the use of a diffusion-weighted MRI technique called 'Constrained Spherical Deconvolution' (CSD) [28]. Of ten 
healthy participants, CSD data was used to create an atlas of the tongue muscles. It showed that the personalized models correctly predicted $80 \%$ of standardized movements whereas, without CSD, only $50 \%$ were predicted correctly.

In this study, we aimed to implement CSD data to create pre- and post-operative personalized FE models [28] with the use of our virtual surgery tool [17]. The individual's pre- and post-operative mobility was assessed by measuring their tongue Range of Motion (in-vivo ROM) using optical tracking [15]. Next, the invivo ROM was used to determine if the predicted ROM was improved by the use of a personalized model.

To assess the effect of the tumor on the pre-operative in-vivo ROM, we compared it to the in-vivo ROM of a previous study. Variations in the extent of virtual surgery, mimicking the real partial glossectomy, were addressed by two observers.

\subsection{METHODS}

\section{Participants}

A total of 11 tongue carcinoma patients were included between August 2018 and March 2019. The following inclusion criteria were used: patients older than 18 years, T-stage $1-3$. All patients were treated with a partial glossectomy. The main exclusion criterion was a previous history of oral or oropharyngeal cancer. Patients who were not eligible for MRI, e.g. due to claustrophobia, shortness of breath while lying down, or pregnancy, were also excluded.

Patients were asked to participate in this study after their first outpatient visit. Before surgery, a diffusion-weighted MRI was made [29]. The ROM was determined using optical tracking [15]. Immediately after surgical resection, the tongue specimen was scanned using a micro-CT scanner to calculate the volume removed from the tongue. ROM measurements were repeated six months after surgery (Figure 7.1).

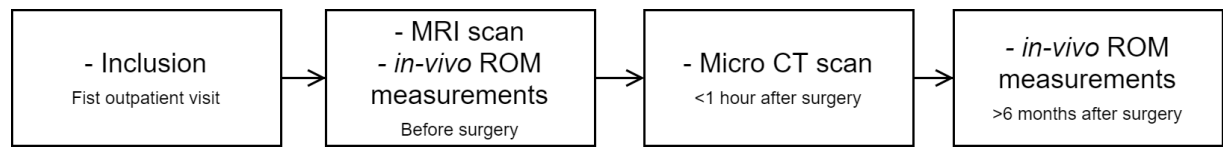

Figure 7.1 - Timeline of patient measurement procedures.

All procedures involving patients were in accordance with the ethical standards of the Medical Ethical Committee of the Netherlands Cancer Institute as well as the Declaration of Helsinki (1964) and later amendments. Written informed consent was obtained from every participant. 
Available clinical data were used to construct both pre-operative generic and personalized models. Subsequently, virtual surgery based on perioperative drawings in the patient's file was performed by two observers with a medical background using both models as described by Kappert et al. [17]. One head-and neck-surgeon (AJMB) confirmed that the incisions created and subsequent postoperative models were in line with the perioperative drawings.

\section{Generic model}

The generic model was constructed following Kappert et al. [17] and Artisynth [30]. The muscle anatomy and shape (3D mesh) of this model was adopted from the model by Buchaillard et al. [21] (Figure 7.2A). Based on this shape a coarse FE structure of cubic hexahedral elements was generated. The 3D mesh of the tongue was embedded in the FE model. This technique ensured quick and easy modification of the FE model, which is a prerequisite for virtual surgery.

The mechanical properties were the same as in previous iterations of the model. The viscoelastic nature of the tongue was modelled by a (hyperelastic) 5-parameter moony Rivlin material model with $C_{10}: 1037$ and $C_{20} 486$ based on ex-vivo research by Gerard et al. [19] and fine-tuned by Buchaillard et al. [21]. The rest of the parameters were set to zero. In addition, a density of $1040 \mathrm{~kg} / \mathrm{m}^{3}$ and Rayleigh damping coefficients $\alpha=40 \mathrm{~s}^{-1}$ and $\beta=0$ were used.

To simulate muscle contraction, externally applied stresses were used to deform the elements and to generate the effect of muscle contraction, as provided by the build-in function by Artisynth [30]. This function also enabled muscle stiffening based on the activation level [31]. Incompressibility was modelled by using a high bulk modulus of $100 C_{10}$. 

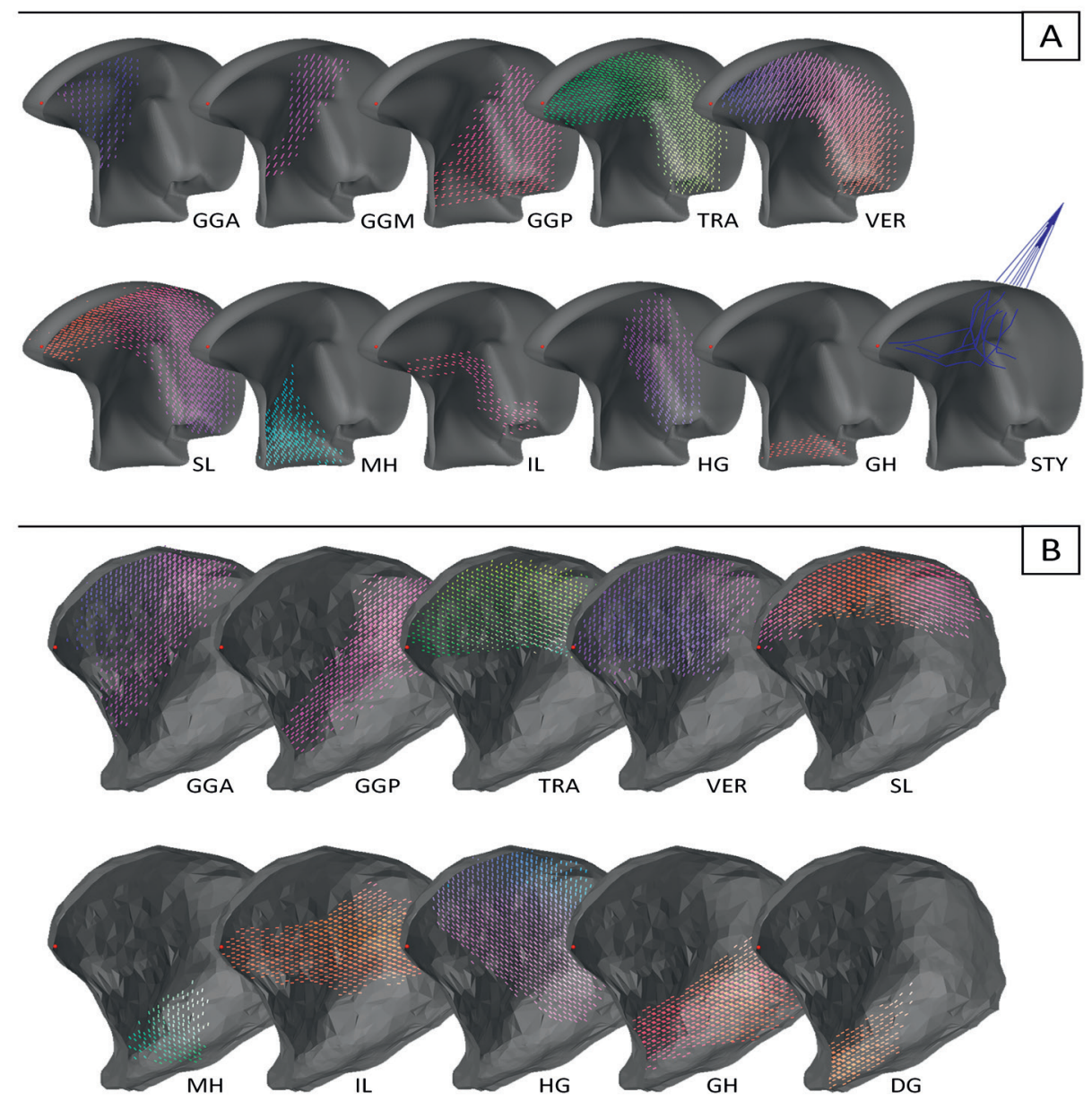

Figure 7.2 - A: The vector fields resembling muscle location and direction of the generic model. Red is anterior-posterior, green is lateral, blue is cranial-caudal. B: The vector fields resembling muscle location and direction of the atlas used in the personalized models. Muscle abbreviations are: genioglossus, -posterior (GGP), -middle (GGP), -anterior (GGA), geniohyoid muscle (GH), hyoglossus (HG), inferior longitudinal muscle (IL), mylohyoid muscle (MH), superior longitudinal muscle (SL), transverse muscle (TRA), vertical muscle (VER), styloglossus (STY) and digastricus (DG).

\section{Personalized model}

The personalized model utilized the same embedded-mesh approach as the generic model. The muscle fiber tracts in this model were based on tractography data from a diffusion-weighted MRI technique called Constrained Spherical Deconvolution (CSD). CSD enables crossing fibers to be visualized and is therefore especially suitable for visualization of tongue musculature. However, due to the susceptibility to high noise levels and artifacts, we created an atlas based on ten healthy volunteers, which was morphed back to that of a single patient (using the acquired diffusion-weighted images) to render high-quality, personalized fiber 
tracks. The technique for acquiring CSD images has been described at length in the literature $[28,32]$.

The tracks acquired using CSD were converted into vector fields resembling the shape and the direction of the muscle at every point in space (Figure 7.2B). In Artisynth, these vectors were converted to muscle elements, similar to the generic model.

Although the genioglossus (GG) in the generic model was divided into three parts [21], the GG of the personalized model was split into two. This concept is based on the location of the short tendon as described by Mu and Sanders [33]: distinct anatomical markers to split the GG into three are lacking. Visualization of the styloglossus was not possible in the atlas [32] and it was not used in the personalized model. This likely does not affect the ROM since the styloglossus is largely used for backward motion. In contrast to the generic model, the anterior bellies of the digastricus muscles were present in the personalized model. The effect of the different muscle bundles for both models was analyzed in Appendix C.

\section{Virtual surgery}

The embedded-mesh approach of the models enabled not only automatic generation of FE models but also real-time editing. The virtual surgery process was the same as in a previous publication [17] and can be summarized as follows:

With the virtual surgery method, the mesh of the generic or personalized model can be edited using a few tools. The excision wound can be delineated in a clockwise manner by selecting multiple points on the mesh (Figure 7.3A). With sliders, the depth of the incision, and the shape of the points halfway to the bottom of the incision can be adjusted to mimic the shape of the excision. The volume and dimensions of the incision are displayed. When the shaping of the surgical incision is finished, an FE model is generated according to the shape of the mesh using the same method as previously described. The muscle vector fields from either the generic or personalized model are embedded in the new FE model, except now at the location of the incision.

Using point-forces distributed over the edge of the incision and halfway, the incision is closed analogously to primary closure using stitches (Figure 7.3B, C). The point-forces are moved to the virtual plane that divide the wound over its longest cross-section. The force of these point forces can be manually adjusted. This results in realistic deformation of the model around the incision. The last step is to affix the virtual stitches into place and to allow the model to reach a steadystate without the point forces 'pulling'. The deformed mesh and muscle vector fields are preserved to simulate the ROM in the post-operative state. A vector 
field describing the extent of the defect is saved to define the area of fibrosis in the post-operative model (Figure 7.3D).

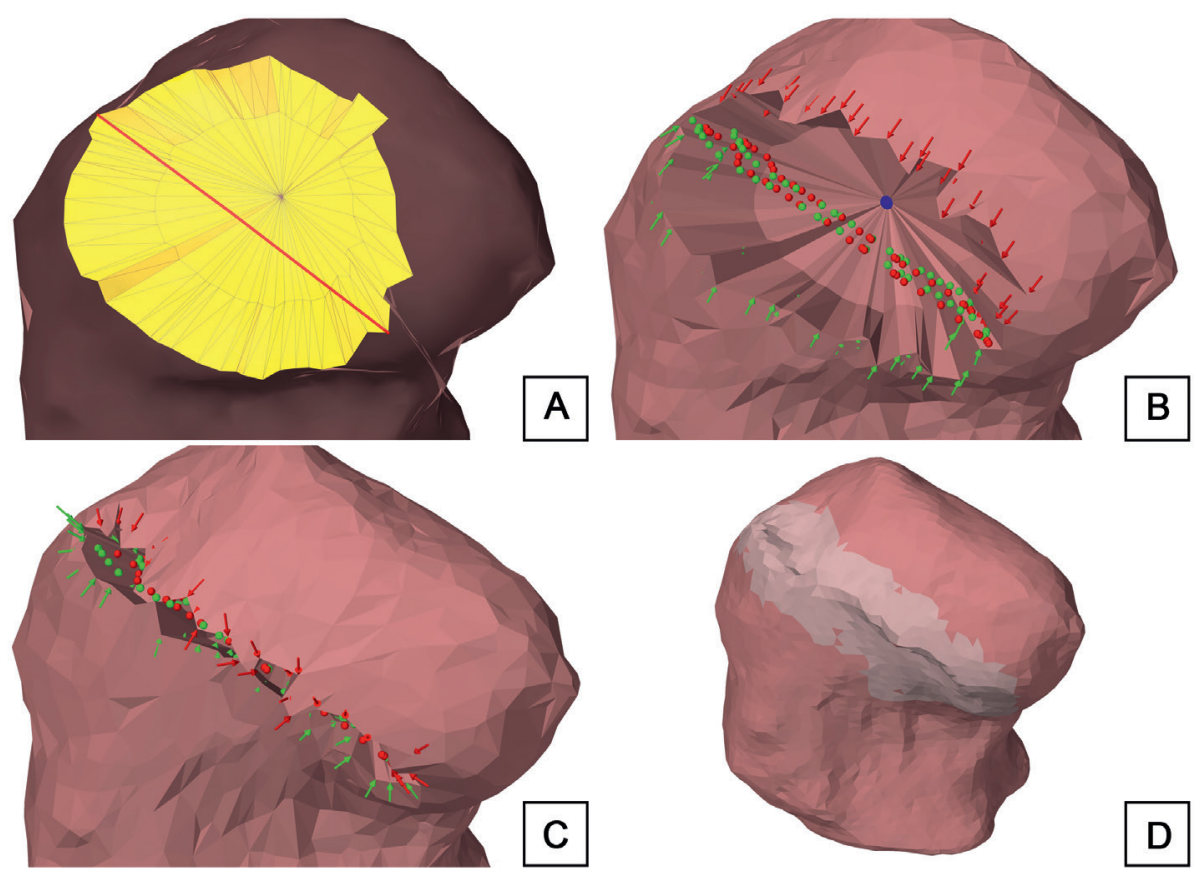

Figure 7.3-A:The excision wound (inyellow) is delineated by selecting multiple points on the mesh. Also, the depth and height can be adjusted. B, C: Using point-forces distributed over the edge of the incision and halfway (green and red arrows), the incision is closed analogously to primary closure using stitches. D: The area of post-operative fibrosis is calculated and represented using a lighter shade of pink.

\section{Validation using optical tracking of range of motion}

To validate the simulated movements of the models, measurements from an optical tracking system were then used. The optical tracker is based on a triplecamera system introduced in the work by van Dijk et al. [16] and improved upon by Kappert et al. [15].

To validate the capability of our models in mimicking the effects of surgery on tongue mobility, in-vivo pre- and post-operative tongue ROM measurements were performed on each participant. Using the triple-camera system and facial landmarks, the tongue tip of the patient was tracked in space and time while performing four tongue maneuvers: protrusion, and moving leftwards, rightwards, and downwards.

From these tongue trajectories, the protrusion-range, left-range, right-range, and down-range were extracted and compared with the mobility ranges of the tongue models. Two times the standard deviation of $3.5 \mathrm{~mm}$ was used to reflect 
the uncertainty as measured in the work by Kappert et al. [15] of pre-operative, in-vivo ROM measurement.

To compare between ROMs after (virtual) surgery, the residual ROM was calculated, which is the post-operative ROM shown as a percentage of the preoperative ROM (reference-state). For the post-operative, in-vivo ROM, the variance was multiplied by two to account for the uncertainty of both the pre- and post-operative measurements, resulting in a coefficient of variation $\left(C_{v}\right)$ of $9.9 \%$. Both the standard deviation and $\mathrm{C}_{\mathrm{v}}$ are shown as grey overlays in the graphics depicting results. The up movement was not used since it cannot be measured reliably.

\section{Observer variability of virtual surgery}

Surgical excision, particularly partial glossectomy, is usually performed without image-based navigation. The size, shape, and method of stitching an actual surgical wound are never the same [34]. In the virtual surgery method, too, every observer and every session also produces slightly different results. In an attempt to minimize variability, two observers ran through the virtual surgery procedure three times for every participant using drawings from the surgical report. One head-and-neck surgeon (AJMB) was consulted to confirm whether the procedures were performed accurately. Before interpreting the final results of this paper, the variation caused by the manual nature of the operation was assessed. The variability was quantified by the coefficient of variation $\left(C_{v}, E q 7.1\right)$ of the ROM movements, simulated by the specific post-operative models.

$$
c_{v}=\frac{\mu}{\sigma}
$$

Where $\mu$ is the mean distance (of the ROM) and $\sigma$ the standard deviation.

The $C_{v}$ was calculated for every combination of movement, patient, and observer. To minimize variability, the result is presented by taking the median of the virtual surgery simulations of both observers, which is why the $C_{v}$ for the observers was also combined was calculated.

\section{Volume of removed (tumor) tissue}

Using a micro-CT, scans were made of the resected specimen and subsequently segmented to obtain the volume. We succeeded in obtaining these scans in eight patients. For the three remaining patients, volumes were estimated based on the (three) dimensions obtained from the pathology report. The volumes (V) were estimated as an ellipsoid based on the measurements obtained: 


$$
V_{\text {resection }}=\frac{4}{3} \pi\left(\frac{a b c}{8}\right)
$$

With $a, b, c$ representing the three dimensions obtained from the pathology report. By estimating the volumes for all patients and comparing these to the available micro-CT data, we found that they did not exactly match up. Based on this difference, a correction factor of 0.69 was calculated to compensate for the deviation.

Since the personalized model was based on patients' imaging data, its size was similar to the tongue size of the individual patient. The generic model, conversely, represents an average tongue size. The ratio between the generic model and the resection is therefore off and needs to be corrected for by scaling the volume (V) of the resected tissue to the size of the generic model:

$$
V_{\text {corrected }}=\frac{V_{\text {tongue model }}}{V_{\text {tongue patient }}} * V_{\text {removed tissue }}
$$

\subsection{RESULTS}

\section{Participant characteristics}

Three patients were excluded from the initial study population of 11 participants: two patients underwent a combined mandibulectomy and neck dissection instead of the scheduled partial glossectomy and one patient refused the diffusionweighted MRI scan. Eventually, we were able to include eight partial glossectomy patients. The baseline and tumor characteristics are summarized in Table 7.1.

For the eight participants included in the study, ROM was assessed both preand post-operatively. However, due to motion artifacts, the obtained diffusionweighted MRI images were of insufficient quality in six patients. Only the data from the two remaining patient datasets were used to create generic and personalized biomechanical models.

\section{Volumes of the tongue and specimen}

Table 7.1 shows that the volumes of the tongues derived from the MRI scans varied considerably ( $>50 \%$ in volume). It also shows the resected tissue based on ellipsoid calculation (Eq 7.2), the measured volume using micro $\mathrm{CT}$, the derived volumes calculated based on the differences between micro-CT (Eq 7.3), and the ellipsoid calculation. The standard deviation of the differences between micro-CT and the ellipsoid calculation is $1.98 \mathrm{~cm}^{3}$. Only datasets of the highlighted patients 6 and 8 , from now on referred to as P1 and P2, were complete and could be used for the construction of personalized models. However, these models both lack 
micro-CT data for the calculation of the volume of removed tissue. Therefore, the ellipsoid measurement with the correction factor is used to estimate the resected volume. For the generic model, the volumes of the removed tissue are corrected for the volume difference between the generic model and tongue and are shown in the last column.

Table 7.1 - Volume of the tongue and removed tissue in $\mathrm{cm}^{3}$. By comparing the results of the ellipsoid (calculated using Eq 7.2) with the micro-CT data a correction factor was calculated to be used in patients from whom the micro-CT data is missing. The relative volume is calculated using Eq 7.3.

\begin{tabular}{|c|c|c|c|c|c|}
\hline \multirow[t]{2}{*}{ Patient \# } & \multirow{2}{*}{$\begin{array}{l}\text { Tongue } \\
\text { volume } \\
\text { (MRI) }\end{array}$} & \multicolumn{4}{|c|}{ Removed tissue volume based on: } \\
\hline & & $\begin{array}{l}\text { Ellipsoid } \\
\text { (Eq 7.2) }\end{array}$ & Micro-CT & $\begin{array}{l}\text { Ellipsoid } \\
\text { multiplied by } \\
\text { correction factor: } \\
0.69\end{array}$ & $V_{\text {corrected }}(\mathrm{Eq} 7.3)$ \\
\hline 1 & 151 & 39.6 & 25.35 & 27.4 & 18.2 \\
\hline 2 & 97 & 23.1 & 18.0 & 16.0 & 20.1 \\
\hline 3 & 173 & 14.7 & - & 10.2 & 6.4 \\
\hline 4 & 124 & 8.8 & 11.8 & 6.1 & 10.3 \\
\hline 5 & 115 & 22.0 & 14.6 & 15.2 & 13.8 \\
\hline 6 (P1) & 99 & 21.1 & - & 14.6 & 16.0 \\
\hline 7 & 109 & 9.1 & 6.3 & 6.3 & 6.3 \\
\hline $8(P 2)$ & 136 & 38.3 & - & 26.5 & 21.2 \\
\hline
\end{tabular}

\section{Range of motion}

To investigate any inconsistencies between the present and previous study [15] in both healthy individuals and post-surgery patients, we compared pre- and postoperative in-vivo ROM with our earlier findings (Table 7.2).

Table 7.2 - Comparison between current and previous study of the total ROM in millimeters [15].

\begin{tabular}{lllll}
\hline & \multicolumn{2}{c}{ Current study } & \multicolumn{2}{l}{ Kappert 2019 [15] } \\
\cline { 2 - 5 } & $\begin{array}{l}\text { Pre-surgery, } \\
\mathrm{n}=8\end{array}$ & $\begin{array}{l}\text { Post-surgery } \\
\text { approximately } \\
6 \text { months, } \mathrm{n}=8\end{array}$ & Healthy $\mathrm{n}=19$ & $\begin{array}{l}\text { Post-surgery } \\
(>6 \text { months, } \\
\mathrm{n}=19)\end{array}$ \\
\hline $\begin{array}{l}\text { Total range of motion: } \\
\text { Left + right + out + down }\end{array}$ & $\begin{array}{l}165 \mathrm{~mm} \\
(126-191)\end{array}$ & $\begin{array}{l}122 \mathrm{~mm} \\
(76-150)\end{array}$ & $\begin{array}{l}162 \mathrm{~mm} \\
(124-196)\end{array}$ & $\begin{array}{l}124 \mathrm{~mm} \\
(87-170)\end{array}$ \\
\hline
\end{tabular}

The mean post-surgerical, total in-vivo ROM of the current study was only marginally different from the 19 post-surgery patients who were measured by in the work by Kappert et al. [15]. The pre-operative, in-vivo ROM was also within range of the in-vivo ROM in healthy participants. 


\section{Clinical characteristics of P1 and P2}

The tumor details of $\mathrm{P} 1$ and $\mathrm{P} 2$ are summarized in Table 7.3.

Table 7.3 - Tumor characteristics of patients P1 and P2 (formerly pnt 6 and 8). The relative volume of the removedtissuewasusedinthegenericmodels, andtherealtumorsizewasusedinthepersonalizedmodels.

\begin{tabular}{lll}
\hline Patients & P1 & P2 \\
\hline Tongue volume & $99 \mathrm{~cm}^{3}$ & $136 \mathrm{~cm}^{3}$ \\
\hline Estimated removed tissue (personalized) & $14.6 \mathrm{~cm}^{3}$ & $26.5 \mathrm{~cm}^{3}$ \\
\hline Relative volume of the removed tissue (generic) & $16.0 \mathrm{~cm}^{3}$ & $21.2 \mathrm{~cm}^{3}$ \\
\hline Tumor stage & $\mathrm{T} 3$ & $\mathrm{~T} 2$ \\
\hline Tumor location & Left & Right \\
\hline
\end{tabular}

\section{Variability}

Table 7.4 shows the coefficient of variation $C_{v}$ for both observers. The combined $\mathrm{C}_{\mathrm{v}}$ is generally below $4 \%$. The peaks in $\mathrm{C}_{\mathrm{v}}$ do not appear to be systematic. The low performance in the personalized model of $\mathrm{P} 1$ is especially noticeable. Bothobservers showed difficulties with reproducing the resection of the T3tumor, which is reflected by the combined $C_{v}$ (13.6\%) of both observers in the personalized model of $\mathrm{P} 1$.

Table7.4-Thecoefficientofvariation $C_{v}$ ofobservers 1,2, and bothobserverscombinedforthemovements of P1andP2. ThefirsteightrowsshowC foreachobserverindividuallyforeachspecificparticipantinboth personalized and generic models. The $\mathrm{C}_{v}$ s of both observers combined are displayed in the last for rows.

\begin{tabular}{|c|c|c|c|c|c|c|c|}
\hline & Patient & $C_{v}$ & Out & Down & Left & Right & Combined \\
\hline \multirow{4}{*}{ Observer 1} & \multirow{2}{*}{ P1 } & $\mathrm{G}$ & $7.4 \%$ & $12.7 \%$ & $1.3 \%$ & $0.9 \%$ & $4.9 \%$ \\
\hline & & $P$ & $8.8 \%$ & $16.5 \%$ & $7.0 \%$ & $19.6 \%$ & $13.1 \%$ \\
\hline & \multirow{2}{*}{ P2 } & $G$ & $4.2 \%$ & $3.9 \%$ & $2.6 \%$ & $0.6 \%$ & $2.7 \%$ \\
\hline & & $P$ & $2.8 \%$ & $1.8 \%$ & $3.1 \%$ & $2.8 \%$ & $2.7 \%$ \\
\hline \multirow{4}{*}{ Observer 2} & \multirow{2}{*}{ P1 } & G & $4.9 \%$ & $3.2 \%$ & $1.1 \%$ & $4.1 \%$ & $3.1 \%$ \\
\hline & & $P$ & $10.8 \%$ & $6.5 \%$ & $1.4 \%$ & $4.1 \%$ & $8.7 \%$ \\
\hline & \multirow{2}{*}{ P2 } & $G$ & $26.1 \%$ & $3.7 \%$ & $0.9 \%$ & $1.3 \%$ & $5.2 \%$ \\
\hline & & $P$ & $4.6 \%$ & $2.4 \%$ & $1.2 \%$ & $4.3 \%$ & $2.7 \%$ \\
\hline \multirow{4}{*}{ Combined } & \multirow{2}{*}{$\mathrm{P} 1$} & $G$ & $6.4 \%$ & $9.5 \%$ & $1.1 \%$ & $3.4 \%$ & $4.7 \%$ \\
\hline & & $P$ & $9.9 \%$ & $19.3 \%$ & $10.5 \%$ & $13.2 \%$ & $13.6 \%$ \\
\hline & \multirow{2}{*}{ P2 } & $G$ & $17.4 \%$ & $5.2 \%$ & $1.8 \%$ & $0.9 \%$ & $4.7 \%$ \\
\hline & & $P$ & $3.6 \%$ & $2.4 \%$ & $2.2 \%$ & $3.8 \%$ & $2.9 \%$ \\
\hline
\end{tabular}

Pre-operative measurements \& simulations:

The pre-operative, simulated, and in-vivo ROMs (Figure 7.4) serve as a reference state for the post-operative measurements (Figure 7.5). The in-vivo ROM is about two times larger than the simulated ROM, but the rest of the comments will focus 
on the relative differences between movements. The generic model was the same for both P1 and P2 and the simulated ROMs were therefore the same. The pre-operative, simulated ROMs for the personalized models were different and showed a ROM of P2 that was slightly larger than P1 in most directions, but less pronounced than in the in-vivo ROM. However, when taking the standard deviation for the in-vivo ROM into account, the pre-operative movements of P1 and P2 were significantly different with the exception of the downward movement. This difference was not visible in the simulated ROM of the personalized model. There was also no significant asymmetry in the lateral pre-operative movements of the in-vivo ROM.
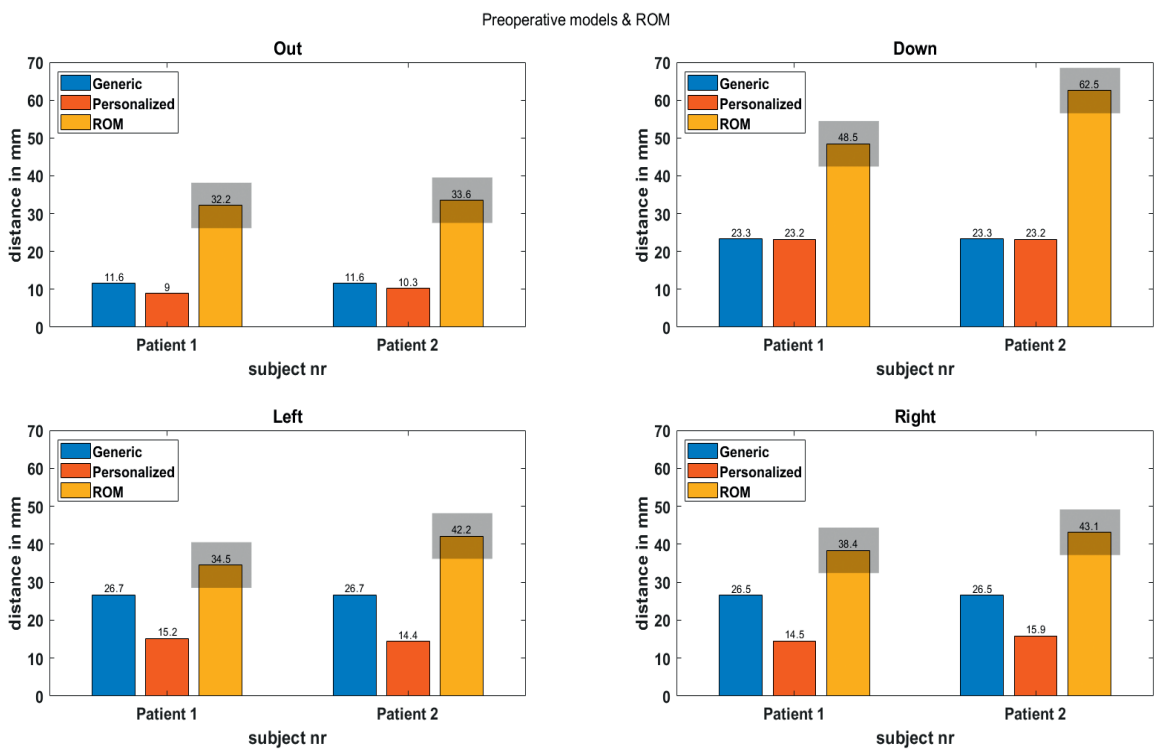

Figure 7.4 - In-vivo ROMs and simulated ROMs for the outward, downward, leftward, and rightward movements. The in-vivo ROM is depicted in yellow and the standard deviation of $3 \mathrm{~mm}$ in gray. The ROMs of the generic and personalized models for $\mathrm{P} 1$ and $\mathrm{P} 2$ are marked in blue and red, respectively.

\section{Post-operative measurements \& simulations}

In Figure 7.5, the residual post-operative in-vivo ROMs and the residual of the simulated ROMs after virtual surgery are shown in percentages. The mean residual, based on the four movements of P2's in-vivo ROM P2 (54\%), was smaller than that of P1's (67.7\%).

In the outward movement, $\mathrm{C}_{\mathrm{v}}$ overlapped between P1 and P2 for both models and the in-vivo ROM, meaning that both models correctly showed there was no significant difference between P1 and P2. For the downward movement, the invivo $\mathrm{ROM}$ of $\mathrm{P} 1$ was larger than $\mathrm{P} 2$. This was also be observed in the personalized model, although the large $C_{v}$ of $P 1$ caused the $C_{v}$ to overlap slightly. The generic 
model simulated a larger residual ROM for P2, which was not seen in-vivo. For the leftward movement, P2 was larger than P1. P2 (right-sided tumor) demonstrated restricted tongue movement to the right, in contrast to $\mathrm{P} 1$ (left-sided tumor), which demonstrated restrictions to the left. Both the generic and personalized models showed less overall impairment compared to the in-vivo ROM, despite having shown an improvement of ROM in the leftward movement of the personalized model of P2. Overall, the differences in the simulated ROM between P1 and P2 were more pronounced in the personalized models and shared more similarities with the in-vivo ROM than the generic models did.
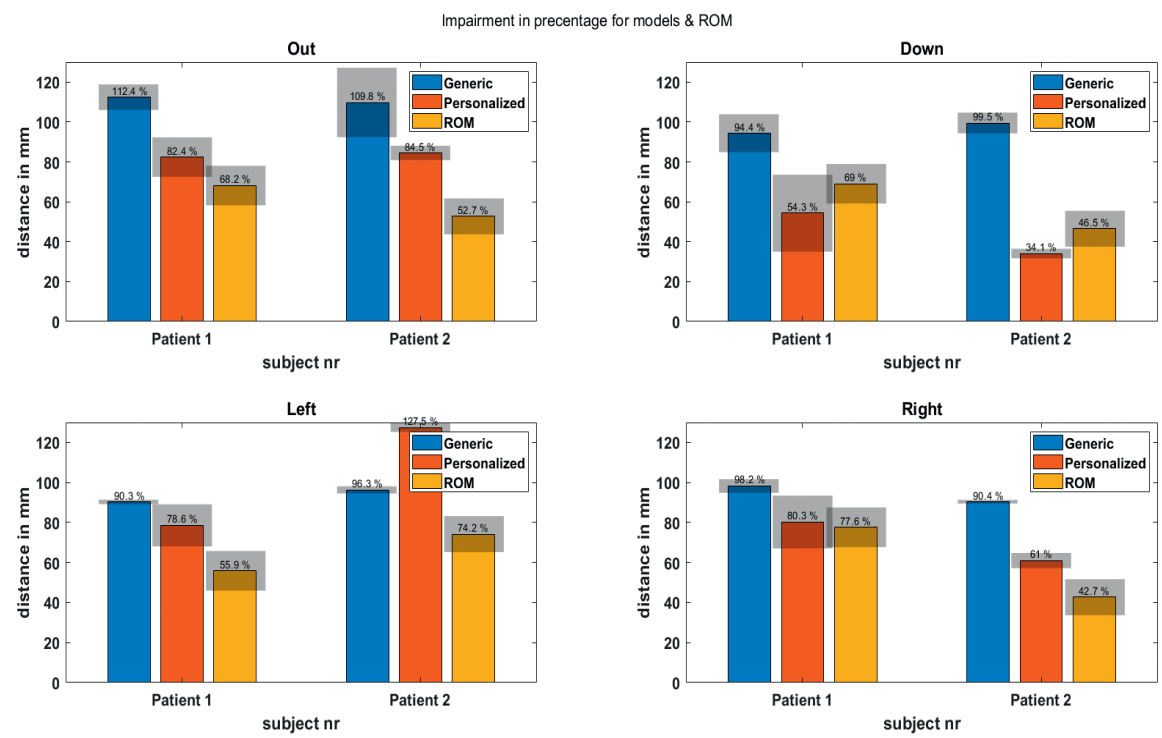

Figure 7.5 - The residual ROM for the outward, downward, leftward, and rightward movements, expressed in the percentage of the ROM before surgery (reference state). The simulated ROM of the generic model is blue, the simulated ROM of the personalized model is red, and the in-vivo ROM is yellow. The calculated $\mathrm{C}_{\mathrm{v}}$ for each specific patient and simulated movement is depicted as a grey bar. The standard deviation of the in-vivo ROM is also shown in grey.

\subsection{DISCUSSION}

This study was the first to show the simulation of pre- and post-operative tongue mobility using personalized biomechanical models. The models were assessed using pre- and post-operative ROM measurements in patients. The inter- and inter-observer variability was assessed to ensure fair comparison between the generic and personalized models. Unfortunately, due to MRI artifacts, only two patient datasets could ultimately be used for analysis. Although this prevented definitive conclusions, the experiments still gave us a clear insight for the strategy of using biomechanical models that require relatively low effort in clinical practice. 


\section{Range of Motion}

The analysis confirms the repeatability of the in-vivo ROM procedure that was introduced by van Dijk et al. [16]. A difference of $38 \mathrm{~mm}$ was seen between healthy participants and post-operative patients. A significant finding was that the preoperative ROMs of patients and healthy participants were indistinguishable (only three $\mathrm{mm}$ difference). Given the substantial size of some of the tumors in this study, it is a clear sign that the maximum ROM does not necessarily affect the size of the tumor. However, this observation may not hold up for more complex movements as only simple reproducible movements were tested. Because of the limited effect on the tongue ROM, it seems not a prerequisite to include the tumor in the simulation process.

\section{Variability}

The variability of the measurements highlighted a general concern when simulating surgery of the tongue. Virtual surgery involving soft tissue without navigation is subject to inaccurate documentation. Next to visual tumor extensions, manual palpation of the tumor margins plays an important role at the deeper level of the incision in order to achieve tumor-free margins. This last part was not displayed in the surgical reports. On top of this, the two observers had difficulties doing 3D drawings based on the 2D surgical sketches from the patients' files. The tongue, deformable and without fixed landmarks as it is, might be one of the most challenging organs to simulate surgically. The effectiveness of biomechanical modelling relies, therefore, to a great extent on future innovations in surgical navigation to ensure accurate tracking of the incisions around the tumor.

\section{Pre-operative simulations}

We recently presented simulations using personalized biomechanical models of healthy participants [28], successfully showing distinctive differences between individuals. Since only two personalized models could be used in the current study, it is hard to confirm our results. Only two out of four movements demonstrated distinctive differences between P1 and P2 in the personalized models. The downward movement of the personalized model was not different from the one in the generic model and the leftward movement was worse than the in-vivo ROM. It is therefore not currently possible to definitively prove that personalized pre-operative models of the tongue improve the level of prediction. Given the lack of differences in in-vivo ROMs between healthy participants and patients pre-operatively, it is plausible to assume that increasing of the number of study participants will lead to similar results in future studies.

\section{Post-operative simulations}

Both patients show the largest lateral impairment in ROM towards the side of the resection, which was the left side and right side for $\mathrm{P} 1$ and $\mathrm{P} 2$, respectively. 
This effect was successfully predicted by the simulations of both the generic and personalized models. The downward and outward residual ROMs were not predicted correctly by both the generic and personalized models. The residual ROM of the personalized models is generally closer to the in-vivo residual ROM, showing more pronounced impairment than in the generic models. Despite the observed trend, the small study size once again prevents us from recommending the personalized model over the generic model at this point.

Some simulations demonstrated an exceptionally improved post-operative ROM, up to $124 \%$ in the case of the personalized left movement of P1. This is counterintuitive, but can be explained by the fact that tissue (model elements) removal results in less resistance during the movement. Especially if none of the muscles needed for a specific motion are removed, it is likely that the tongue movements will be more extended. Simulation of fibrosis by stiffening elements could also have side effects. In the human tongue, fascial planes affect the mobility of the tongue by providing additional support to muscles [35]. Since these structures are absent in the model, the stiffening of elements could create a crowbar-effect, resulting in an increased ROM.

\section{Limitations}

Trying to simulate the motion of the most complex muscle in the human body comes with understandable limitations. First, it is not yet known exactly how the tongue works in every respect. While Mu and Sanders' research discussed key aspects of the innervation, the exact distribution of motor units in the tongue, neuro-muscular interactions, and muscle divisions are not fully understood [36]. In addition to this, tissue parameters cannot be fully explained using the conventional constitutive models, and are yet to be determined [22,37].

In the current study, we created models based on limited data due to unexpected artifacts in the diffusion-weighted MRIs. The CSD protocol that resulted in useful imaging of healthy participants [28] did not reach a similar level in patients. The B0-inhomogeneities were presumably caused by the lower volumes of the tongue after surgery. Because post-operative scanning took place at the end of the day, we expect that fatigue made it harder for the patients to avoid making (in)voluntary tongue movements. As diffusion-weighted MRI is more sensitive for artifacts than regular MRI, we could not use $75 \%$ of the data gathered. For future research, the protocol needs to be improved by shortening the scan time, by giving better instructions, and/or by using tools to limit or restrict tongue movement. The effect of B0-inhomogeneity on the lower volume needs to be assessed as well. 
In three out of the eight patients, we did not succeed in obtaining a micro-CT image of the specimen. Unfortunately, this include the two patients who were used to create our post-operative models. While the calculated conversion from the pathology report was accurate for the other six patients, with a standard deviation of only $1.98 \%$, it still creates extra uncertainty when judging the data. The volume of the resections for the generic model was corrected for the size of the tongue because these could vary by more than $50 \%$. This means that even for the generic model, segmentation of the tongue would be required to create a model with a relatively comparable resection size. This favors the use of the personalized model since the manual work required by both methods is about the same. However, tissue shrinkage was not been taken into account in this study, and this could have lead to an underestimation of the removed tissue volume. Studies report that tissue volume can end up 30\% smaller just before final pathology reporting, of which the first $20 \%$ of the shrinkage occurs during the resection phase [38]. For larger tumors (T2-T3), this is significantly less.

Surgical wounds were drawn in 3D using a free-hand tool and perioperative drawings. While this is visually comparable to real surgery, it lacks feedback regarding the exact anatomical location of the tumor. Accuracy in drawing the surgical wound could be improved by visually overlaying the tumor in augmented reality. In the future, presurgical planning and surgical navigation could significantly improve the precision of the virtual incision and 3D wound dimensions, thus minimizing the mismatch between real surgery and virtual surgery.

In addition to the inaccuracy of the model and method, we also have to consider the difficulty in simulating the real surgical procedure. Perioperative drawings of the incision are not accurate in terms of exact dimensions and the template in the patient's file at our institute only allows for a drawing of a certain orientation in 2D. Several unpublished projects on the use of intraoral scanning at our institute tried to capture the 3D shape of a surgical wound, but the deformability of tissues and the reflective quality of blood have thus far prevented useable images from being captured.

\section{Future perspectives}

This study gave us a glimpse into the future of what plannable and predictable surgery could look like. It also indicated the significant hurdles that first need to be overcome before biomechanical modeling of post-operative function can live up to its potential.

The movement and deformation of the FE models are dictated by constitutive models that describe tissue behavior. The model used in our study does not 
account for the complex viscoelastic behavior of the tongue but instead simplifies the tissue to a rubber-like (hyperelastic) material. With this simplified approach, the constants that describe the model are not even well-defined [22,37]. This makes the evidence-based simulation of tongue tissue a difficult task, with many unknown variables. New developments in the description of tongue tissue and treatment-induced fibrosis are essential for the future of biomechanical modeling.

Based on what we learned from this study, it is expected that the imaging of muscle fibers will be important in the development of future models. While CSD proved to be very useful in Kappert et al. [28] when imaging healthy participants, postoperative imaging was only suitable for further analysis in $25 \%$ of the included patients. Improvements to this technique are required to make it robust enough to be usable in everyday clinical practice. More ideally, further improvements of diffusion-weighted MRI techniques and CSD could eliminate the need for an atlas to extract a detailed image of fiber tracks.

It is not possible to predict the outcome of surgical intervention if the geometry of the real surgical resection is not accurately mimicked in the virtual model. The implementation of proper surgical navigation is a prerequisite for attaining an optimal surgical simulation. Only with the use of this tool (and proper imaging of the tumor margins) in an augmented reality setting, can improved simulation of surgical intervention be achieved, resulting in a better prediction of the expected functional outcome. Because of the deformability of the tongue, surgical navigation in an of itself is an arduous task. Even without a model, surgical navication alone could theoretically contribute to greater surgical efficiency in achieving tumorfree resection margins with less unnecessary removal of adjacent healthy muscle structures. Only after this breakthrough occurs can biomechanical modeling of the tongue find its way into clinical practice.

\section{Conclusion}

We used CSD and virtual surgery to create models simulating the post-operative state of the tongue after a partial glossectomy. Due to artifacts in the diffusionweighted MRI, post-operative models could only be created for two out of the eight patients, resulting in an underpowered study that demonstrates possible trends but prevents us from drawing definitive conclusions. We are inclined to state that the two personalized models are indicative of simulating more pronounced impairment of certain movements, which are closer to the in-vivo ROM in patients than was observed in the generic models. The many assumptions needed to create models speak to the need for additional research on tissue parameters, imaging modalities, and surgical navigation. Hopefully, biomechanical modeling of the tongue will contribute to better patient counseling and pre-operative outcome prediction in the future. 


\subsection{REFERENCES}

1. Gatta G, Botta L, Sánchez MJ, Anderson LA, Pierannunzio D, Licitra L, et al. Prognoses and improvement for head and neck cancers diagnosed in Europe in early 2000s: The EUROCARE-5 population-based study. Eur J Cancer. 2015;51: 2130-2143. doi:10.1016/j.ejca.2015.07.043

2. UK Cancer Research. Head and neck cancers incidence statistics [Internet]. 2019 [cited 26 Apr 2019]. Available: https://www.cancerresearchuk.org/healthprofessional/cancer-statistics/statistics-by-cancer-type/head-and-neck-cancers/ incidence\#heading-Four

3. Warnakulasuriya S, Greenspan JS. Epidemiology of Oral and Oropharyngeal Cancers BT. In: Warnakulasuriya S, Greenspan JS, editors. Textbook of Oral Cancer: Prevention, Diagnosis and Management. Cham: Springer International Publishing; 2020. pp. 5-21. doi:10.1007/978-3-030-32316-5_2

4. Kreeft AM, Van Der Molen L, Hilgers FJ, Balm AJ. Speech and swallowing after surgical treatment of advanced oral and oropharyngeal carcinoma: A systematic review of the literature. Eur Arch Oto-Rhino-Laryngology. Springer; 2009;266: 1687-1698. doi:10.1007/s00405-009-1089-2

5. Van Der Molen L, Van Rossum MA, Jacobi I, Van Son RJJH, Smeele LE, Rasch CRN, et al. Pre- and posttreatment voice and speech outcomes in patients with advanced head and neck cancer treated with chemoradiotherapy: Expert listeners' and patient's perception. J Voice. Elsevier Ltd; 2012;26: 664.e25-664.e33. doi:10.1016/j.jvoice.2011.08.016

6. Costa Bandeira AK, Azevedo EHM, Vartanian JG, Nishimoto IN, Kowalski LP, CarraraDe Angelis E. Quality of life related to swallowing after tongue cancer treatment. Dysphagia. 2008;23: 183-192. doi:10.1007/s00455-007-9124-1

7. Lee DY, Ryu YJ, Hah JH, Kwon TK, Sung MW, Kim KH. Long-term subjective tongue function after partial glossectomy. J Oral Rehabil. 2014;41: 754-758. doi:10.1111/ joor.12193

8. Husaini H, Krisciunas GP, Langmore S, Mojica JK, Urken ML, Jacobson AS, et al. A survey of variables used by speech-language pathologists to assess function and predict functional recovery in oral cancer patients. Dysphagia. 2014;29: 376-386. doi:10.1007/ s00455-014-9520-2

9. Montero PH, Patel SG. Cancer of the oral cavity. Surg Oncol Clin N Am. 2015/04/15. 2015;24: 491-508. doi:10.1016/j.soc.2015.03.006

10. Kerawala C, Roques T, Jeannon JP, Bisase B. Oral cavity and lip cancer: United Kingdom National Multidisciplinary Guidelines. J Laryngol Otol. Cambridge University Press; 2016;130: S83-S89. doi:10.1017/S0022215116000499

11. Sessions DG, Spector GJ, Lenox J, Haughey B, Chao C, Marks J. Analysis of treatment results for oral tongue cancer. Laryngoscope. 2002;112: 616-25. doi:10.1097/00005537-200204000-00005

12. Wong TSC, Wiesenfeld D. Oral Cancer. Aust Dent J. 2018;63: S91-S99. doi:10.1111/ adj.12594

13. Kreeft AM, Tan IB, Leemans CR, Balm AJM. The surgical dilemma in advanced oral and oropharyngeal cancer: how we do it. Clin Otolaryngol. 2011;36: 260-266. doi:10.1111/ j.1749-4486.2011.02299.x

14. Pfister DG, Spencer S, Brizel DM, Burtness B, Busse PM, Caudell JJ, et al. Clinical practice guidelines in oncology. Head Neck Cancers Version. 2019;1. 
15. Kappert KDR, van Alphen MJA, Smeele LE, Balm AJM, van der Heijden F. Quantification of tongue mobility impairment using optical tracking in patients after receiving primary surgery or chemoradiation. Kimple RJ, editor. PLoS One. 2019;14: e0221593. doi:10.1371/journal.pone.0221593

16. van Dijk S, van Alphen MJA, Jacobi I, Smeele LE, van der Heijden F, Balm AJM. A New Accurate 3D Measurement Tool to Assess the Range of Motion of the Tongue in Oral Cancer Patients: A Standardized Model. Dysphagia. Springer US; 2016;31: 97-103. doi:10.1007/s00455-015-9665-7

17. Kappert KDR, van Alphen MJA, van Dijk S, Smeele LE, Balm AJM, van der Heijden F. An interactive surgical simulation tool to assess the consequences of a partial glossectomy on a biomechanical model of the tongue. Comput Methods Biomech Biomed Engin. 2019;22: 827-839. doi:10.1080/10255842.2019.1599362

18. Wilhelms-Tricarico RF, Perkell JS. Biomechanical and Physiologically Based Speech Modeling BT - Progress in Speech Synthesis. In: van Santen JPH, Olive JP, Sproat RW, Hirschberg J, editors. New York, NY: Springer New York; 1997. pp. 221-234. doi:10.1007/978-1-4612-1894-4_17

19. Gerard JMM, Ohayon J, Luboz V, Perrier P, Payan Y. Non-linear elastic properties of the lingual and facial tissues assessed by indentation technique: Application to the biomechanics of speech production. Med Eng Phys. Elsevier; 2005;27: 884-892. doi:10.1016/j.medengphy.2005.08.001

20. Gerard J-M, Wilhelms-Tricarico R, Perrier P, Payan Y, Gérard J-M, Wilhelms-Tricarico R, et al. A 3D dynamical biomechanical tongue model to study speech motor control. Res Dev Biomech. 2003;1: 49-64.

21. Buchaillard S, Perrier P, Payan Y. A biomechanical model of cardinal vowel production: muscle activations and the impact of gravity on tongue positioning. J Acoust Soc Am. 2009;126: 2033-2051. doi:10.1121/1.3204306

22. Hermant N, Perrier P, Payan Y. Human Tongue Biomechanical Modeling. In: Payan Y, Ohayon JBT-B of LO, editors. Biomechanics of Living Organs: Hyperelastic Constitutive Laws for Finite Element Modeling. Oxford: Academic Press; 2017. pp. 395-411. doi:10.1016/B978-0-12-804009-6.00019-5

23. Buchaillard S, Brix M, Perrier P, Payan Y. Simulations of the consequences of tongue surgery on tongue mobility: Implications for speech production in post-surgery conditions. Int J Med Robot Comput Assist Surg. 2007;3: 252-261. doi:10.1002/ rcs.142

24. Gérard J-M, Perrier P, Payan Y. 3D biomechanical tongue modeling to study speech production. Speech Prod Model Phonetic Process Tech. 2006; 85-102. Available: http://hal.archives-ouvertes.fr/hal-00108521/

25. Wilhelms-Tricarico R. Development of a tongue and mouth floor model for normalization and biomechanical modelling. Proceedings of the Fifth Speech Production Seminar and CREST Workshop on Models of Speech Production. Kloster Seeon, Bavaria; 2000. pp. 141-148.

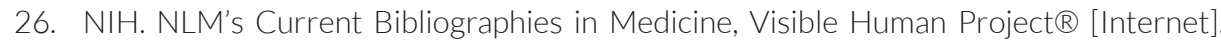
2007 [cited 3 Jul 2018]. Available: http://www.nlm.nih.gov/archive/20120907/pubs/ cbm/visible_human_2007.html

27. Fujita S, Dang J, Suzuki N, Honda K. A Computational Tongue Model and its Clinical Application. Oral Sci Int. 2007;4: 97-109. doi:10.1016/S1348-8643(07)80004-8 
28. Kappert KDR, Voskuilen L, Smeele LE, Balm AJM, Jasperse B, Nederveen AJ, et al. Personalizedbiomechanicaltonguemodelsbasedondiffusion-weightedMRIandvalidated using optical tracking of range of motion. Manuscript submitted for publication. 2020;

29. Voskuilen L, Mazzoli V, Oudeman J, al. E. Crossing muscle fibres in the tongue resolved using constrained spherical deconvolution. International Society of Magnetic Resonance in Medicine. 2017.

30. Lloyd JE, Stavness I, Fels S. ArtiSynth: A Fast Interactive Biomechanical Modeling Toolkit Combining Multibody and Finite Element Simulation. Studies in Mechanobiology, Tissue Engineering and Biomaterials. 2012. pp. 355-394. doi:10.1007/8415_2012_126

31. Blemker SS, Pinsky PM, Delp SL. A 3D model of muscle reveals the causes of nonuniform strains in the biceps brachii. J Biomech. 2005;38: 657-665. doi:10.1016/j. jbiomech.2004.04.009

32. Voskuilen L, Mazzoli V, Oudeman J, Balm AJM, van der Heijden F, Froeling M, et al. Crossing muscle fibers of the human tongue resolved in vivo using constrained spherical deconvolution. J Magn Reson Imaging. John Wiley \& Sons, Ltd; 2019;50: 96-105. doi:10.1002/jmri.26609

33. Sanders I, Mu L. A three-dimensional atlas of human tongue muscles. Anat Rec. 2013;296: 1102-1114. doi:10.1002/ar.22711

34. Ansarin M, Bruschini R, Navach V, Giugliano G, Calabrese L, Chiesa F, et al. Classification of GLOSSECTOMIES: Proposal for tongue cancer resections. Head Neck. 2019; doi:10.1002/hed.25466

35. Abd-El-Malek S. Observations on the morphology of the human tongue. J Anat. 1939;

36. Mu L, Sanders I. Human tongue neuroanatomy: Nerve supply and motor endplates. Clin Anat. 2010;23: 777-791. doi:10.1002/ca.21011

37. Kappert KDR, Connesson N, Elahi SA, Boonstra S, Balm AJM, van der Heijden F, et al. In-Vivo Tongue Elasticity Measured By Aspiration: Resting vs General Anesthesia. Manuscript submitted for publication. 2020;

38. Mistry RC, Qureshi SS, Kumaran C. Post-resection mucosal margin shrinkage in oral cancer: Quantification and significance. J Surg Oncol. 2005; doi:10.1002/jso.20285 

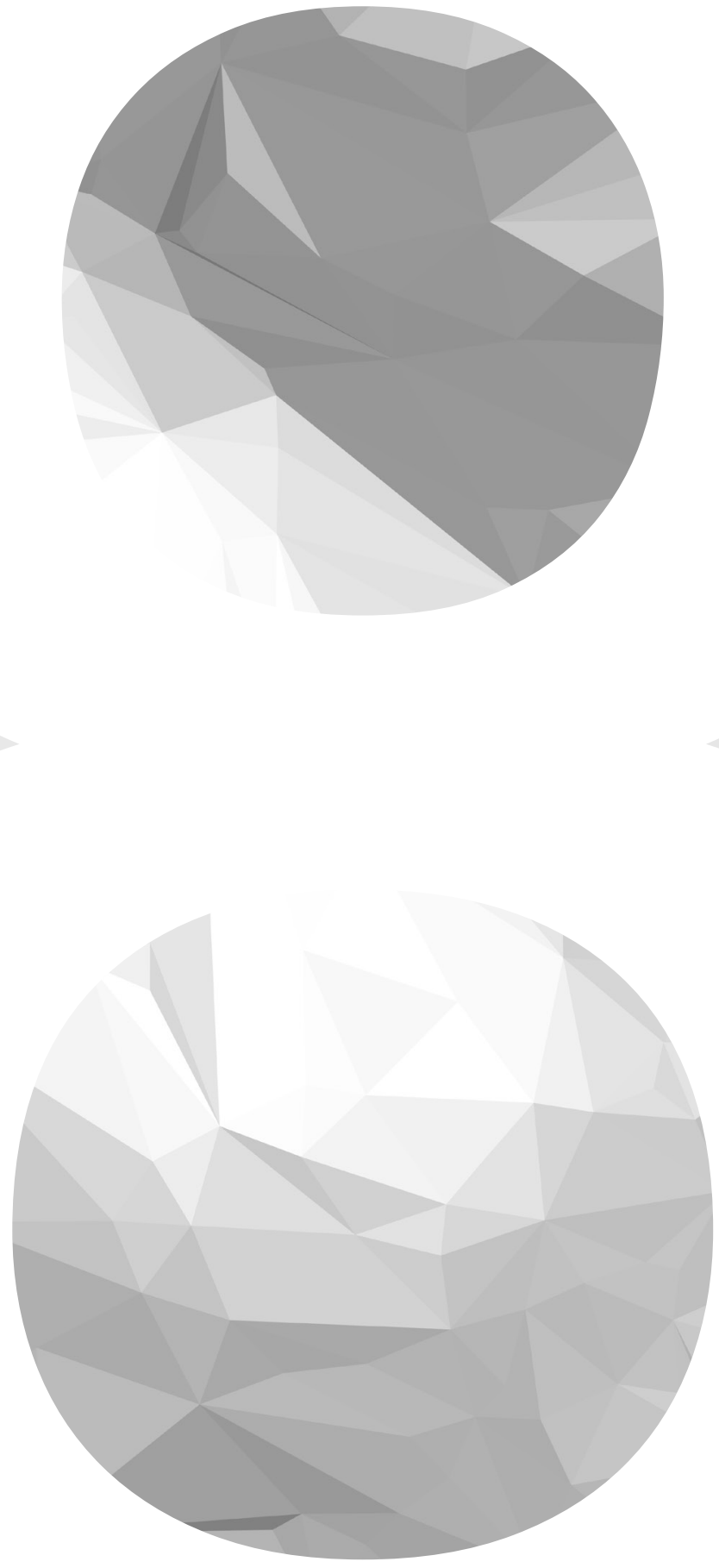
SUMMARY AND

CONCLUSION 


\subsection{ENGLISH SUMMARY}

\section{Chapter 1}

Tongue cancer is not a common disease but treatment can have a devastating effect on speech, swallowing, and mastication. Surgery is currently the treatment of choice. Organ sparing alternatives such as radiotherapy or chemoradiation are resorted to in situations of anatomical or functional inoperability. The first type of inoperability includes cases in which surgery would affect vital organs, leading inevitably to serious or even life-threatening defects. The second type of inoperability refers to functional problems that are considered unacceptable for the patient. Assessing the extent of functional problems after surgery is complex, in part due to the anatomical and functional complexity of the organ itself: the interdigitating muscles, complex innervation and control, tissue characteristics, and the varying effects of fibrosis after treatment make predicting individual tongue function impossible based on experience and reasoning alone.

The aim of the Virtual Therapy project is to develop a Digital Twin model based on the actual physics and anatomy of the tongue. In a clinical workflow, this model could theoretically assist in predicting functional loss, thereby assisting the physician and patient to better understand the effects of treatment on function.

This thesis provided a glimpse in what we can expect from a Digital Twin and encompassed surgical simulation, optical tracking, tongue gymnastics, tissue properties, muscle fiber track imaging, and simulation of tongue mobility after partial glossectomy.

\section{Chapter 2}

In order to simulate post-operative function using a biomechanical model, a tool to perform "virtual surgery" is needed. While there are several software packages available to create biomechanical models, no software currently allows for a simulation of the resection procedure in primary surgery. Therefore we created a tool within the Artisynth modeling platform (University of British Columbia, Vancouver, Canada) based on a previous model by the TIMC-IMAG Laboratory at the University of Grenoble, France. Movement and deformation in these models are calculated using the finite element (FE) method, which divides a large structure, such as the tongue, into smaller parts (elements) that are easier to calculate. In this new approach, muscle fiber directions are embedded in the elements to effectuate contraction into the direction of the muscle fiber upon activation. A unique feature of the newly created model is that cubic-shaped elements can be changed dynamically to alter shape. Resections can be mimicked with relative ease by clicking several points on the tongue model surface in a clockwise manner, followed by finetuning of the depth and shape of the resection. In the next step, 
the edges of the created incision are moved towards each other to simulate a suturing procedure. To allow for deformation of tissue during this procedure, a pre-operative FE model was created without elements, nodes, or muscle bundles inside the created surgical incision. During the last step, the tissue surrounding the incision is stiffened to simulate fibrosis. The strength of this procedure lies in the realistic deformation of muscle bundles inside the tongue (elements), as in real surgery. It was shown that the new approach enabled virtual surgery on an existing biomechanical model without seriously impacting the motion of the model during simulation of movements.

A simulated partial glossectomy based on a single clinical case showed that the model demonstrated the same type of impairment as observed in a post-operative video recording of the patient. The extent of impairment was partially determined by the degree to which fibrotic tissue is simulated. While the results are promising, this research also shows the urgent need for more objective methods to measure tongue motion, tissue, fibrosis properties, and tongue anatomy.

\section{Chapter 3}

To validate the performance of biomechanical models, the simulated movements need to be related to the post-operative movement of the patient in-vivo. A previously developed method to measure 2D Range of Motion (ROM) proved to be promising. Because the full 3D capabilities of the system were not used, it was not able to detect or correct for the angle of the tongue and jaw.

We developed a new method that placed markers on the tongue, jaw, and face of the patient. These markers were tracked in 3D while the patient performed outward, downward, upward, leftward, and rightward movements. The trajectory of the tongue was corrected for the position of the head and jaw and subsequently projected onto a $3 \mathrm{D}$ visualization of the head.

In Chapter 3, this new method was used to measure the ROM of 19 healthy participants, 18 post-chemoradiation, and 17 post-surgery patients. For every participant and patient, the ROM distance and the deflection angle of the tongue into a specific direction was measured. The technique showed an excellent intraand interobserver variability (ICC $>0.9$ for all measures) and good reproducibility with a maximum standard deviation of $3.5 \mathrm{~mm}$. The ROM was the smallest and the deflection the largest in post-surgery patients. Chemoradiation patients showed a ROM in between healthy participants and post-surgery patients. No distinctive asymmetry was noticed based on the tumor side or location. 
Research focusing on the validation of the biomechanical tongue using pre- and post-operative ROM measurements, as described in Chapters 6 and 7, benefited from this new technique.

\section{Chapter 4}

Chapter 4 takes a step back to focus on a relatively simple clinical feature with possibly large implications. Although never confirmed, it stands to reason that above-average preoperative mobility will result in better postoperative function. This is based on the fact that innervation of tongue muscles is known to differ among individuals, which suggests that the compensatory mechanisms after surgery might be related. This study, therefore, assessed the ability to perform five tongue movements as rolling, twisting (two sides), folding, and the 'cloverleaf' in a healthy population.

Pre-operatively, however, a tumor might restrict a patient from performing such movements. Whether a subject can recall if they can perform specific tongue movements is therefore important. To assess memory of movements and actual movements, two observers interrogated a group of 387 healthy participants, representative of the Dutch population. Participants were asked if they could perform certain tongue movement(s), after which they were asked to actually perform those movement(s). The percentage of people who could perform a certain movement was: rolling: $83.7 \%$, cloverleaf: $14.7 \%$, folding: $27.5 \%$, twisting left: $36.1 \%$, and twisting right: $35.6 \%$. Comparison with the literature showed a distinctive difference in the number of people who can fold the tongue (3\% versus $27.5 \%$ in this study). Various conditional correlations were made and showed, for example, that the ability to roll the tongue is not a prerequisite for folding of the tongue. Of the participants, $9.9 \%$ and $13.1 \%$ incorrectly assumed that they could demonstrate tongue rolling and cloverleaf. Tongue folding and twisting (left or right) were incorrectly assumed in $36.9 \%, 24.1 \%$, and $25.4 \%$ of the cases.

A strong recommendation for assessing tongue mobility before surgery was hard to make, as the two movements that people were most aware of (rolling and cloverleaf) were also the movements that occurred very often or very infrequently. This means that there are a large number of people who can perform rolling but cannot perform cloverleaf. This limits the number of patients that could benefit from the model and poses a potential problem for future study designs.

\section{Chapter 5}

One of the more pivotal aspects of FE simulation is the description of its material behavior. Most important in this respect are the characteristics of different tongue tissues and fibrotic changes after treatment. Tongue tissue in-vivo exhibits in general a nonlinear, time-dependent, and inhomogeneous behavior that is 
significantly different from tissue ex-vivo. Tensile tests are the golden standard to determine the material parameters but cannot be performed in vivo. Using a suction-based technique, it is possible to measure the deformation of tissue in vivo using a vacuum that is created inside a cup placed on the tongue mucosa. Measuring the pressure and removed volume reveals the aspirated tissue. Inverse modeling of this situation in a FE simulation can approach the parameters that are needed for the constitutive equation that describes the tissue characteristics. However, in living persons, muscle tone is always present at rest (AR). It was hypothesized that this muscle tone would be reduced in a state of general anesthesia (GA).

To measure the tissue characteristics AR and under GA, 10 patients with healthy tongues scheduled for gynecologic or urologic surgery were included. The measured pressure relative to the tissue shape change revealed that tissue under GA was stiffer than while awake in eight of the 10 participants. This significant difference is also visible in the Young's modulus determined using the inverse FE simulation: a mean of $1638 \mathrm{~Pa}$ AR versus 3060 under GA.

Various hypotheses about the effects of anesthetics affecting tissue stiffness administered during GA, i.e. blood flow, perfusion, and upper airway reflexes, have been proposed but could not be confirmed. The method and analysis itself were critically reassessed and it was confirmed that no systematic error could have caused the difference between AR and GA.

While this research was initially aimed at finding a state of the tongue without muscle tone, it resulted in the discovery of a state with a much higher tissue stiffness than had been expected. The method itself proved to be reliable during in-vivo conditions AR, but the values of an absolute rest state of the tongue remain unelucidated.

\section{Chapter 6}

The size of the tongue can differ among humans: sometimes it can be more than twice the size of another individual. For accurate prediction of function loss using a Digital Twin model, personalization of the model is key. In Chapter 6, the aim was to personalize biomechanical models for 10 healthy subjects utilizing muscle fiber track data obtained with an MRI technique called Constrained Spherical Deconvolution(CSD).

A single MRI does not contain enough information to create detailed fiber tracks for a single subject. Therefore, the data of a single subject is registered to an average or atlas, created with 10 (other) previously scanned subjects. The displacement field obtained from this registration was subsequently used to morph the atlas into a personalized muscle description of the tongue. 
Personalized models for all 10 subjects were created by taking the technique used in Chapter 2 to create a biomechanical model and by changing the generic muscle bundles for these personalized muscle bundles. Via inverse modeling, the tongue model was instructed to move the tongue-tip outwards, downwards, leftwards, and rightwards as far as possible, thereby calculating the muscle activations needed to achieve these positions.

As explained in Chapter 3, a comparison with the ROM of all ten subjects showed that the personalized models predicted the ROM correctly in $80 \%$ of the cases after a correction factor was applied. Without personalization, only $50 \%$ of the cases were predicted correctly. It was striking to observe that the biomechanical models were not able to reach the same magnitude of ROM as was observed invivo. To compensate for this phenomenon, a correction factor was calculated. A challenge that remains when creating FE models of the tongue is to show large deformations without becoming numerically unstable.

\section{Chapter 7}

In Chapter 7, we used our knowledge from the previous chapters to test the level of prediction of mobility after partial glossectomy by the use of a personalized biomechanical tongue model. The generic and personalized models of Chapter 6 and the surgical simulation tool of Chapter 2 were validated by optical tracking described in Chapter 3.

For this study, eight patients were included immediately after having been diagnosed with tongue cancer. Preoperatively they underwent MRI (CSD) imaging and a ROM measurement. Within an hour after the surgical resection, the specimen was scanned in a micro-CT scan to obtain the 3D dimensions of the surgical wound. At a minimum of six months after surgery, the patient performed an additional post-operative ROM measurement. Unfortunately, only two out of eight MRI scans were of sufficient quality to be used for creating a personalized biomechanical model, due to unexpected artifacts caused by $\mathrm{BO}$ inhomogeneity and involuntary movements. Therefore, the subsequent analysis could only be performed for two subjects.

Using the patient files, two observers were instructed to recreate the surgical wound three times on both the generic and personalized models of the participants using the surgical tool as described in Chapter 2. As is true for real surgery of the tongue, creating the same incisions is difficult given the absence of clear landmarks of the tongue.

Inverse simulations of all the generic and personalized models created by the two observers were performed. The tongue tip was made to move outwards, 
downwards, leftwards, rightwards and upwards. For the subsequent analysis, the mean simulated ROM of the multiple models of both observers was taken.

The results show that the personalized model can simulate more distinctive movement impairments than the generic model. However, because only two patient models could be created for comparison, no definitive conclusion could be drawn.

In addition to the fact that the simulated ROMs cannot reach the same magnitude of ROM in-vivo (Chapter 6), the imaging technique appears not yet ready for applications to predict tongue mobility post-surgically.

\section{Conclusion and future perspectives}

One decade after the initiation of the Virtual Therapy project, this is the third thesis bringing us closer to the creation of a Digital Twin to predict function loss and help guide physicians and patients in the clinical decision-making process. The tongue is a vital organ essential for many important functions of daily life, such as speech, swallowing, and mastication. Damage to this organ has serious consequences for quality of life and physiological well-being. The complex structures, innervation, and tissue properties make the tongue one of the most difficult organs to investigate - but this complexity also forecloses opportunities for research.

This thesis is characterized by exploring unknown territory at high speed. By pursuing the goal of creating personalized postoperative prediction models, various interesting topics have been touched upon that are now available for further scientific research. From the research conducted as part of this thesis, we have learned that a functioning biomechanical model and tools of interaction are relatively straightforward to implement, but that personalization poses the real challenge.

Imaging modalities are essential for the representation of muscle anatomy and innervation. Diffusion-weighted MRI and techniques such as CSD (Chapter 6) showed potential to accurately represent tongue anatomy, but are currently very sensitive for motion artifacts or result in very long scan times. Improving these techniques is key for the further development of biomechanical tongue models. Used in combination with (waterproof and high density) surface electromyography, action potentials could be followed and subsequently used to map innervation patterns in the tongue.

For accurate deformation of the tongue and fibrous tissue, a solid mathematical description of the tissue parameters is essential. Measurements in a steady- 
state situation are needed to obtain the parameters of tongue tissue in rest. We know that this state is not present in the ex-vivo situation, but in this thesis, we also learned that it is not present in the in-vivo situation under general anesthesia caused by effects that are currently unknown. This still leaves the scientific community with the question of how to approach the exact tissue properties of the tongue. In addition, an effort was made to use ultrasound elastography to determine differences between fibrotic and non-fibrotic tissue. This was not pursued further after it became apparent that the inhomogeneity and many muscle directions made it difficult to perform reliable measurements. However, with the new technique introduced in Chapter 5 , it might be possible in the future to measure the relative difference between fibrous and normal tissue.

Despite these many fundamental challenges, we cannot forget the initial goal of creating a reliable method for predicting function loss to help guide the physician and patient in their decision-making. In order to make progress in the short term, multiple phases with clinically applicable models or decision aids are needed, starting with a low grade of personalization, and gradually working towards a fully personalized model. This requires a focus on the translation of mobility measures of the model to actual function. In this first step, different defined cases can be showcased and validated against real function. For example, such a case might show a range of functional effects of a tumor at the left base of the tongue or a tumor at the tip of the tongue. Various techniques - not only limited to biomechanical modeling - can then be used to optimize the prediction. A hybrid of a biomechanical model with a statistical algorithm could, in addition to the physical simulation of post-operative motion, also account for other details that cannot be simulated, such as patient characteristics, comorbidity, or the ability to perform specific tongue movements, such as rolling and cloverleaf (Chapter 4).

Advanced 3D tracking techniques, such as electromagnetic articulography or 3D dynamic MRI could also be used to obtain more detailed tongue motion during talking, swallowing, or mastication. In a more advanced biomechanical model, simulation of a solid or fluid bolus in a post-operative model could show the implications for mastication and swallowing. Speech syntheses could deform preoperative speech to match the speech of the modeled post-treatment outcome. These same techniques can be used to predict the effects of rehabilitation and training. While pursuing the ideas of this ambitious project, it is important to show progress and set short-term goals as guideposts on the road towards the creation of a true Digital Twin. In this thesis, we got a glimpse of what this journey might look like, and the next step is to continue down this road. 


\subsection{NEDERLANDSE SAMENVATTING}

\section{Hoofdstuk 1}

Tongkanker is geen veelvoorkomende ziekte, maar de effecten van de behandeling kunnen bij gevorderde ziekte desastreuze gevolgen hebben voor spraak, slikken en eten. Chirurgie is op dit moment de meest voorkomende behandeling. Maar er kan ook worden gekozen voor orgaansparende behandelingen, zoals radiotherapie of chemoradiatie bij anatomische of functionele inoperabiliteit. Het eerste type inoperabiliteit betreft de situatie waarin een operatie vitale organen aantast, met hoogstwaarschijnlijk levensbedreigende schade tot gevolg. Het tweede type betreft functionele problemen die voor de patiënt onaanvaardbaar zijn. Het beoordelen van deze functionele problemen is met name lastig vanwege de complexiteit van het orgaan. De kruisende spiervezels, de complexe innervatie en interactie met het brein, de weefseleigenschappen en de slecht voorspelbare effecten van fibrose na een behandeling maken het onmogelijk om de postoperatieve tongfunctie te voorspellen op basis van alleen kennis en ervaring.

Het Virtual Therapy project heeft de ambitie om een 'digitale dubbelganger' te ontwikkelen op basis van de echte fysica en anatomie van de hoofd-halsregio. In een klinische setting kan dit model helpen bij het voorspellen van functieverlies, waarmee aan de arts en patiënt de effecten van de behandeling op functies getoond kunnen worden.

Dit proefschrift geeft een eerste indruk van wat we kunnen verwachten van een digitale tweeling. Hierbij verkennen we chirurgische simulaties, optische tracking, tonggymnastiek, weefseleigenschappen, beeldvorming van spiervezels en simulatie van tongmobiliteit na een partiële glossectomie.

\section{Hoofdstuk 2}

Om de postoperatieve functie te kunnen simuleren met een biomechanisch model, is een 'tool' nodig om virtuele chirurgie uit te voeren. Hoewel er verschillende softwarepakketten zijn om biomechanische modellen te creëren, biedt geen enkele software de mogelijkheid om de chirurgische resectie te simuleren. Daarom hebben we binnen het Artisynth-modelleringsplatform (University of British Columbia, Vancouver) een tool gecreëerd op basis van een eerder model van het TIMC-IMAG-laboratory van de Universiteit van Grenoble. Beweging en vervorming van deze modellen worden berekend met behulp van de eindige elementenmethode (Finite Element; FE), waarmee een grote structuur, zoals de tong, wordt verdeeld in kleinere delen (elementen). Bij deze nieuwe benadering zijn spiervezelrichtingen ingebed in de elementen om bij activering de contractie in de richting van de spiervezel te bewerkstelligen. Een uniek kenmerk van 
dit nieuwe model is dat kubusvormige elementen dynamisch kunnen worden toegevoegd of verwijderd om de vorm van het eindige elementenmodel aan te passen. Op een gebruiksvriendelijke manier kunnen chirurgische resecties worden nagebootst door simpelweg de resectie op het tongmodeloppervlak te tekenen. Hierna kan ook de diepte en vorm van de resectie worden aangepast. Een nieuw FE-model wordt gecreëerd op basis van de tongvorm met resectie waardoor elementen en spierbundels op de plek van de resectie verdwijnen. In een volgende stap bewegen de randen van de gemaakte incisie naar elkaar toe om zo een hechtingsprocedure te simuleren. Een voordeel van deze procedure is dat de spierbundels in de tong (elementen) vervormen, vergelijkbaar met echte chirurgie. Tijdens de laatste stap wordt het weefsel rond de incisie verstijfd om littekenweefsel na te bootsen. In deze studie hebben we laten zien dat het mogelijk is om virtuele chirurgie toe te passen op een bestaand model, zonder dat de gesimuleerde postoperatieve bewegingen hierdoor beïnvloed worden.

Een gesimuleerde partiële glossectomie op basis van één casus toonde aan dat het model hetzelfde type beperking vertoonde als de patiënt op een postoperatieve video-opname. De mate van beperking wordt beïnvloed door de hoeveelheid en de stijfheid van het gesimuleerde littekenweefsel. Hoewel de resultaten veelbelovend waren, bleek ook dat er veel behoefte was aan meer objectieve manieren om tongbewegingen, weefseleigenschappen, fibrose-eigenschappen en de tonganatomie te verkrijgen.

\section{Hoofdstuk 3}

Om een indruk te krijgen van de prestaties van biomechanische modellen, moesten gesimuleerde bewegingen worden gevalideerd met postoperatieve tongbewegingen van patiënten in-vivo. Een eerder ontwikkelde methode om $2 \mathrm{D}$ Range of Motion (ROM) te meten, was veelbelovend. Echter, omdat de volledige 3D-mogelijkheden van het systeem niet werden gebruikt, kon de hoek van de tong niet worden gedetecteerd en kon er niet voor de stand van de kaak worden gecorrigeerd.

Daarom hebben we een nieuwe methode ontwikkeld met markers op de tong, kaak en gezicht van de patiënt. Deze markers werden in 3D gevolgd terwijl de patiënt afzonderlijke tongbewegingen naar buiten, naar beneden, naar boven, naar links en naar rechts uitvoerde. Het traject van de tong werd gecorrigeerd voor de positie van het hoofd en de kaak en vervolgens geprojecteerd op een 3D-visualisatie van het hoofd.

In hoofdstuk 3 werd de nieuwe methode gebruikt om de ROM te meten van negentien gezonde proefpersonen, achttien patiënten post-chemoradiatie en zeventien patiënten postoperatief. Voor elke deelnemer en patiënt werd de 
ROM-afstand en de hoekafwijking van de tong in een bepaalde richting gemeten. De techniek liet een uitstekende intra- en interobserver variabiliteit (ICC > 0.9 voor alle richtingen) en een goede reproduceerbaarheid met een maximale standaarddeviatie van 3,5 mm zien. De ROM was het kleinst en de hoekafwijking het grootst bij de postoperatieve patiënten. De patiënten post-chemoradiatie vielen tussen de gezonde deelnemers en postoperatieve patiënten in. Er werd geen significante asymmetrie opgemerkt op basis van de tumorzijde of locatie.

Onderzoek gericht op de validatie van de biomechanische tongmodellen door middel van pre- en postoperatieve ROM-metingen, zoals beschreven in de hoofdstukken 6 en 7, profiteerden van deze nieuwe techniek.

\section{Hoofdstuk 4}

Hoofdstuk 4 focust zich op een wat luchtiger onderwerp door te kijken naar simpele klinische eigenschappen van de tong, maar met mogelijk grote implicaties. Hoewel het nooit is bewezen, voelt het als vanzelfsprekend om aan te nemen dat een bovengemiddelde preoperatieve mobiliteit van de tong zal resulteren in een beter postoperatief functioneren. Dit is gebaseerd op het feit dat de innervatie van tongspieren verschilt van persoon tot persoon, wat erop zou kunnen wijzen dat de compensatiemechanismen na een operatie een rol zouden kunnen spelen in het functieherstel. In deze studie werd daarom het vermogen om vijf tongbewegingen uit te voeren beoordeeld. De bewegingen waren: rollen, draaien (twee kanten), vouwen en het maken van een 'klaverblad'.

Preoperatief kan een tumor de patiënt echter belemmeren om een dergelijke beweging uit te voeren. Daarom is het belangrijk dat men zich ervan bewust is dat $\mathrm{hij} / \mathrm{zij}$ bepaalde tongbewegingen ooit heeft kunnen uitvoeren. Om te kijken hoe bewust mensen zijn van hun eigen tongbewegingseigenschappen, ondervroegen twee waarnemers een groep van 387 gezonde deelnemers, representatief voor de samenstelling van de Nederlandse bevolking. Deelnemers werd gevraagd of ze bepaalde tongbeweging(en) konden uitvoeren. Daarna werd verzocht deze daadwerkelijk uit te voeren. Het percentage mensen dat een bepaalde beweging kon uitvoeren was voor rollen: $83,7 \%$, klaverblad: $14,7 \%$, vouwen: $27,5 \%$, naar links draaien: $36,1 \%$ en naar rechts draaien: $35,6 \%$. Als we dit vergelijken met de spaarzame gegevens in de internationale literatuur zien we duidelijke verschillen met het aantal mensen dat de tong kan vouwen (3\% in de literatuur en $27,5 \%$ in deze studie). Door te kijken naar voorwaardelijke kansen toonden we bijvoorbeeld aan dat het kunnen rollen van de tong geen voorwaarde is voor het vouwen van de tong. Van de deelnemers ging $9,9 \%$ en $13,1 \%$ er ten onrechte vanuit dat ze de tong kon rollen of een klaverblad kon vormen. Voor het vouwen van de tong en het draaien naar links of rechts gingen in 36,9\%, 24,1\% en $25,4 \%$ van de gevallen proefpersonen er ten onterechte vanuit dat ze dit konden. 
Er kon moeilijk een sterke aanbeveling worden gedaan om een van de bewegingen te gebruiken voor het beoordelen van de tongmobiliteit vóór een operatie, aangezien de twee bewegingen waarvan mensen zich het meest bewust waren (rollen en klaverblad) ook de bewegingen waren die heel vaak of heel weinig voorkwamen. De groep mensen die wel kan rollen, maar geen klaverblad kan uitvoeren is erg groot ten opzichte van de andere groepen. Dit beperkt het aantal patiënten dat baat zou kunnen hebben bij het model en vormt een potentieel probleem voor toekomstige onderzoeken.

\section{Hoofdstuk 5}

Een van de meest cruciale aspecten van FE-simulatie is de beschrijving van het materiaalgedrag. Het belangrijkste in dit opzicht zijn de kenmerken van verschillende typen tongweefsel en fibrotische veranderingen (verlittekening) na behandeling. Tongweefsel in-vivo vertoont over het algemeen niet-lineair, tijdsafhankelijk en inhomogeen gedrag, dat significant verschilt van de ex-vivo situatie. Testen met een trekbank zijn de gouden standaard als het gaat om de bepaling van materiaalparameters, maar kunnen niet in-vivo worden uitgevoerd. Met behulp van een op zuigkracht werkende techniek is het mogelijk om de vervorming van weefsel in-vivo te meten met behulp van een vacuüm dat wordt gecreëerd in een klein dopje dat op het tongslijmvlies wordt geplaatst. Door het meten van de druk en het verwijderde volume kan de vorm en daarmee de stijfheid van het opgezogen weefsel worden bepaald. Inverse modellering van deze situatie in een FE-simulatie kan de parameters benaderen die nodig zijn voor de constitutieve vergelijking die de weefselkenmerken beschrijft. Bij de levende tong is spierspanning echter altijd aanwezig in rust. De hypothese was dat deze spierspanning minder of afwezig zou zijn onder narcose.

Omdeweefselkenmerken in rusten onder narcose te meten, werden tien patiënten met een gezonde tong, welke in afwachting waren voor een gynaecologische of urologische operatie, geïncludeerd. De gemeten druk ten opzichte van de verandering van de weefselvorm liet zien dat weefsel onder narcose veel stijver was dan in rust bij acht van de tien deelnemers. Dit significante verschil was ook zichtbaar in de Young's (elasticiteits)modulus die was bepaald met behulp van de inverse FE-simulatie: een gemiddelde van $1638 \mathrm{~Pa}$ in rust versus $3060 \mathrm{~Pa}$ onder narcose.

Er is gezocht naar een verklaring door te kijken naar de effecten die anesthetica (die tijdens een narcose worden toegediend) zouden kunnen hebben op weefselstijfheid. Maar verschillende hypotheses over het effect hiervan op bloedstroom, perfusie en reflexen van de bovenste luchtwegen, konden met de verzamelde data in deze studie niet worden getoetst. De methode en analyse 
werden kritisch bekeken om te bevestigen dat geen enkele systematische fout het verschil tussen de waardes in rust en onder narcose heeft kunnen veroorzaken.

Hoewel dit onderzoek aanvankelijk gericht was op het vinden van een toestand van de tong zonder spierspanning, resulteerde het in de ontdekking van een toestand met een veel hogere weefselstijfheid dan verwacht. De methode zelf bleek betrouwbaar te zijn bij in-vivo condities in rust; maar de waarden die de absolute rusttoestand van de tong beschrijven, zijn tot op heden nog niet gevonden.

\section{Hoofdstuk 6}

De grootte van de tong kan bij mensen verschillen. Soms kan de tong van de ene persoon tot meer dan twee keer zo groot zijn dan die van de ander. Voor een nauwkeurige voorspelling van functieverlies met behulp van een 'digitale dubbelganger', is personalisatie van het model essentieel. In hoofdstuk 6 was het doel om biomechanische modellen voor tien gezonde proefpersonen te personaliseren door gebruik te maken van spiervezelrichtingen verkregen met behulp van een MRI-techniek, genaamd Constrained Spherical Deconvolution (CSD).

Een enkele MRI-scan bevat echter niet genoeg informatie om de gedetailleerde spierstructuur van een persoon te verkrijgen. Daarom werden de gegevens van één proefpersoon geregistreerd richting een populatiegemiddelde (of atlas), welke was gemaakt met tien (andere) eerder gescande proefpersonen. Het uit deze registratie verkregen vectorveld werd vervolgens gebruikt om de atlas te vervormen richting een gepersonaliseerd vectorveld van de tongspieren.

Door de techniek uit hoofdstuk 2 te gebruiken om een biomechanisch model te maken en de generieke spierbundels door deze gepersonaliseerde spierbundels te vervangen, werden gepersonaliseerde modellen voor alle tien proefpersonen gemaakt. Via inverse modellering werd het tongmodel geïnstrueerd om de tongpunt zo ver mogelijk naar buiten, naar beneden, naar links en naar rechts te bewegen, waarbij de spieractivaties werden berekend die nodig waren om deze posities te bereiken.

Een vergelijking met de ROM, zoals uitgelegd in hoofdstuk 3, van alle tien proefpersonen toonde aan dat de gepersonaliseerde modellen de ROM correct voorspelden in $80 \%$ van de gevallen nadat een correctiefactor was toegepast. Zonder personalisatie werd slechts in $50 \%$ van de gevallen de ROM correct voorspeld. Opvallend was dat de biomechanische modellen niet zo ver konden reiken als de gemeten tong in-vivo. Om dit fenomeen te compenseren, werd een correctiefactor berekend. Het is nog steeds een uitdaging om FE-modellen van de 
tong te maken die grote vervormingen aankunnen zonder numeriek onstabiel te worden.

\section{Hoofdstuk 7}

In hoofdstuk 7 hebben we de kennis uit de voorgaande hoofdstukken gebruikt om de kwaliteit van de voorspelling van mobiliteit na een partiële glossectomie met behulp van een gepersonaliseerd biomechanisch tongmodel te testen. De generieke en gepersonaliseerde modellen van hoofdstuk 6 en de chirurgische simulatietool van hoofdstuk 2 werden gevalideerd met behulp van de optische tracking methode beschreven in hoofdstuk 3.

Voor deze studie werden acht patiënten direct na het eerste bezoek aan de polikliniek geïncludeerd. Preoperatief ondergingen de patiënten een MRI (CSD-) scan en een ROM-meting. Binnen een uur na de chirurgische resectie werd het gereseceerde weefsel gescand in een micro-CT-scan om de 3D-afmetingen van de chirurgische wond te verkrijgen. Minimaal zes maanden na de operatie onderging de patiënt een aanvullende postoperatieve ROM-meting. Helaas waren slechts twee van de acht MRI-scans van voldoende kwaliteit om te worden gebruikt voor het maken van gepersonaliseerde biomechanische modellen. Dit komt door onverwachte artefacten, veroorzaakt door onvrijwillige bewegingen. De volgende stap kon daardoor slechts voor twee patiënten worden uitgevoerd.

Twee beoordelaars kregen de opdracht om, aan de hand van informatie uit het patiëntendossier, een chirurgische wond te creëren met behulp van de tool voor virtuele chirurgie, zoals beschreven in hoofdstuk 2. Dit deden de beoordelaars drie keer met zowel de generieke als de gepersonaliseerde modellen van de proefpersonen. Net als bij een echte partiële glossectomie, is het moeilijk om de exacte dimensies van de incisie te bepalen door het ontbreken van duidelijke oriëntatiepunten op de tong.

Vervolgens werden er inverse simulaties uitgevoerd met alle generieke en gepersonaliseerde modellen die door de twee beoordelaars geconstrueerd waren. In deze simulaties worden de spierbundelcombinaties berekend die de tongpunt naar buiten, naar beneden, naar links, naar rechts en naar boven laten bewegen. Voor het analyseren van de resultaten werd voor de gesimuleerde ROM's, van de door beide beoordelaars gecreëerde modellen, het gemiddelde bepaald.

De resultaten laten zien dat het gepersonaliseerde model met meer detail een bewegingsbeperking kan simuleren dan het generieke model. Omdat er maar met modellen van twee patiënten simulaties konden worden verricht, kon niet geconcludeerd worden dat het gepersonaliseerde model statistisch significant beter was. 
Naast het feit dat de gesimuleerde ROM's niet dezelfde afstanden van ROM invivo kunnen bereiken (hoofdstuk 6), bleek de techniek om spiervezels te scannen met MRI nog niet voldoende geoptimaliseerd om deze op een betrouwbare manier te gebruiken bij patiënten.

\section{Conclusie \& toekomstvisie}

Een decennium na de start van het Virtual Therapy project, is dit het derde proefschrift dat het project dichter bij de realisatie van een digitale tweeling brengt. Een biomechanisch model met als doel om functieverlies te kunnen voorspellen ter ondersteuning van klinische besluitvorming. De tong is een vitaal orgaan dat essentieel is voor veel belangrijke dagelijkse functies, zoals spreken, slikken en kauwen. Schade aan dit orgaan kan ernstige gevolgen hebben voor de kwaliteit van leven. De complexe structuren, innervatie en weefseleigenschappen maken de tong tot een van de lastigste organen om te onderzoeken. Maar dit biedt ook kansen.

Dit proefschrift kenmerkt zich door het met hoge snelheid verkennen van onbekend terrein. Met het nastreven van het creëren van gepersonaliseerde postoperatieve voorspellingsmodellen, zijn verschillende interessante onderwerpen belicht die in de toekomst verder kunnen worden onderzocht. Van de onderzoeken hebben we geleerd dat een functionerend biomechanisch model en een virtuele chirurgie tool relatief eenvoudig te implementeren zijn, maar dat vooral de stap naar personalisatie uitdagend is.

Beeldvormingstechnieken zijn essentieel voor de weergave van spieranatomie en -innervatie. Diffusie-gewogen MRI en technieken zoals CSD (hoofdstuk 6) toonden potentie om de anatomie nauwkeurig weer te geven, maar zijn in de huidige staat van ontwikkeling erg gevoelig voor bewegingsartefacten of resulteren in zeer lange scantijden. Verbetering van deze technieken is essentieel voor de verdere ontwikkeling van biomechanische tongmodellen. In combinatie met (waterresistente en hoge dichtheid) oppervlakte-elektromyografie kunnen actiepotentialen worden gebruikt om de innervatiepatronen in de tong in kaart te brengen.

Voor een nauwkeurige deformatie van de tong en het bindweefsel tijdens simulatie is een gedegen wiskundige beschrijving van de weefseleigenschappen essentieel. Metingen van de tong zonder aanwezigheid van spierspanning zijn nodig om de parameters van tongweefsel in rust te verkrijgen. Bekend was dat deze parameters niet detecteerbaar waren in de ex-vivo situatie. Dit proefschrift toont aan dat ze ook niet aanwezig zijn in de in-vivo situatie onder algehele narcose als gevolg van verstijving van de tong door een nog onbekende oorzaak. Dit maakt dat de wetenschap nog steeds kampt met de vraag hoe de exacte 
weefseleigenschappen van de tong moeten worden benaderd. Daarnaast is geprobeerd om echo-elastografie te gebruiken om verschillen tussen fibrotisch en niet-fibrotisch weefsel vast te stellen. Dit onderzoek werd echter niet vervolgd nadat bleek dat de inhomogeniteit van het weefsel en de vele spierrichtingen het moeilijk maakten om betrouwbare metingen te doen. Met de nieuwe techniek die in hoofdstuk 5 is geïntroduceerd, is het misschien mogelijk om het relatieve verschil tussen fibreus en normaal weefsel te meten.

Met zoveel fundamentele uitdagingen mogen we het oorspronkelijke doel van het project niet vergeten: het creëren van een methode om patiënt en arts te informeren over het voorspelde functieverlies na een behandeling. We zijn van mening dat op korte termijn vooruitgang geboekt kan worden in meerdere stappen met klinisch toepasbare modellen of keuzehulpen. We stellen ons voor dat we van klinisch toepasbare modellen met een lage mate van personalisatie geleidelijk toewerken naar volledig gepersonaliseerde modellen. Dit vereist extra aandacht voor de vertaling van de mobiliteit van het model naar de daadwerkelijke functie. Verschillende technieken, naast biomechanische modellen, kunnen vervolgens worden gebruikt om de voorspellingen te optimaliseren. Een combinatie van een biomechanisch model met een statistisch algoritme zou, naast de fysieke simulatie van postoperatieve beweging, ook andere details kunnen meenemen die niet kunnen worden gesimuleerd. Bijvoorbeeld patiëntkarakteristieken, comorbiditeit of het vermogen om specifieke tongbewegingen uit te voeren, zoals rollen en het maken van een klaverblad (hoofdstuk 4).

Geavanceerde 3D-trackingtechnieken, zoals elektromagnetische articulografie of 3D-dynamische MRI, kunnen worden gebruikt om ook gedetailleerde tongbewegingen te verkrijgen tijdens praten, slikken of kauwen. Door het simuleren van een vaste of vloeibare bolus in een geavanceerd postoperatief model zou het effect van een behandeling op kauwen en slikken kunnen worden nagebootst. Spraaksynthese zou preoperatieve spraak kunnen vervormen naar postoperatieve spraak met behulp van de gemodelleerde situatie na behandeling. Dezelfde technieken kunnen worden gebruikt om de effecten van revalidatie en training te voorspellen. Bij het nastreven van de ideeën van dit ambitieuze project is het belangrijk om vooruitgang te blijven boeken en kortetermijndoelen te stellen, zodat zichtbaar wordt hoever we af staan van de creatie van een echte digitale dubbelganger. In dit proefschrift hebben we een eerste indruk gekregen van hoe de weg daarnaartoe eruit kan zien, maar de volgende stap is om deze weg ook echt te gaan bewandelen. 


\section{APPENDIX A}

\section{Simulating deformation using FE models}

This subchapter will give a more in-depth view of the modeling aspects that come with creating biomechanical tongue models. Building a Finite element (FE) model from the ground up is complex and time-consuming [1]. Various commercial software solutions provide a framework to create FE models, such as ANSYS, Abaqus, and COMSOL. Also, open-source solutions, such as FEMBio and Artisynth, that are focused particularly on biomechanics, can be used. The biomechanical models used in Chapters 1, 5, and 6 are created using Artisynth. This was a natural choice given the existing cooperation with the creators of Artisynt at UBC Vancouver. This paragraph will explain the basic principles of FE simulations based on the implementation by ArtiSynth. The full documentation of ArtiSynth can be found at www.artisynth.org

\section{FEM}

The building blocks of a FE model are called "elements" (figure A.1). These Elements are built using characteristic points in space called "Nodes". These nodes have certain quantities (stiffness, mass, stress, etc.). Within an element, the so-called "shape-function" describes how these quantities are interpolated within an element. Depending on the type of element, it is either shaped as a tetrahedron, hexahedron, pentahedron, or pyramid in 3D FE modeling. Adjacent elements share their nodes and larger models can use thousands of elements. In the end, an element is a visualization of a part of the volume that is only described by the nodes, and therefore, mathematical speaking, only the nodes exist. For a linear problem, a system of linear algebraic equations (containing the nodes' displacements) should be assembled and solved [2,3].

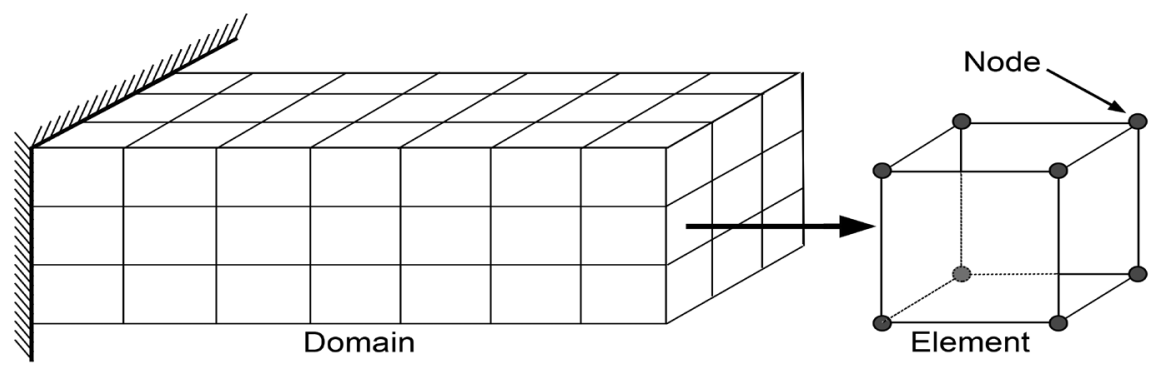

Figure A.1 The domain (or model or object) is divided into finite elements. The domain consists of hexahedral elements using eight nodes. The elements on the left side are constrained. 
The main steps in creating the FE model are:

- Discretize the domain: divide a domain (the object) into finite elements and nodes.

- Develop element equations using the physics of the particular problem.

- Assembling of element equations: Local element equations need to be combined properly to find a global equation system. Element connections are used in this process and also boundary conditions should be imposed.

- Solve the global equation system: Direct and Iterative methods can be used the find nodal values.

Examples of the above method can be found in the lecture notes of [2] or the book of Bonet \& Wood[3].

ArtiSynth combines the above-mentioned method to simulate combined FE and rigid bodies. For future work on the digital twin, this is important since the head and neck functions are often characterized by the combination of rigid (bone) structures and soft (muscle and tissue) structures. The components of an Artisynth simulation are:

- Dynamic components: Components that change due effects of force effectors or constraints. There are 2 types of components in ArtiSynth: a six degrees of freedom (DOF) rigid body and 3 DOF particles, from which the nodes of the FEM models are a subclass off

- Force effectors: Point to point muscles and finite elements

- Constraints: Bilateral constraints (FEM incompressibility and joints), and unilateral constraints (Contact and joint limits)

\section{Physical Simulation: Forward modeling}

A second-order differential equation (ODE) that results from the physics of the mechanical system, as an approximation of the partial differential equation, needs to be solved. After application of the finite element modeling, Newton's second law gives:

$$
\mathbf{M} \dot{\mathbf{u}}=\mathbf{f}(\mathbf{q}, \mathbf{u}, \mathrm{t})
$$

Eq A. 1

$\mathbf{f}(\mathbf{q}, \mathbf{u}, t)$ is the force of all force effector components with $\mathbf{q}$ the positions of the dynamic components, the velocities, and the time. $\mathbf{M}$ is the composite mass matrix wherein FEM uses a lumped mass model to enable contact with other structures such as rigid bodies and mass-springs (e.g. muscle fibers). The bilateral constraints are $\boldsymbol{G}(\boldsymbol{q}) \boldsymbol{u}=0$ and unilateral constraints as $\boldsymbol{N}(\boldsymbol{q}) \boldsymbol{u} \geq 0$. The ODE can be solved by integration forward in time. To simulate this system, a time-discrete 
approximation must be applied to implement the integration. This forward modeling requires that all inputs are available during numerical integration.

\section{Solving the ODE}

Numerical integrating formula 1 with the given constraints $(\boldsymbol{G}(\boldsymbol{q}) \boldsymbol{u}=0$ and $\boldsymbol{N}(\boldsymbol{q}) \boldsymbol{u} \geq 0)$ can be done with both implicit and explicit integrators. FEM models are often solved with an implicit integrator to allow larger time steps and thus to boost the performance. The integrators that can be used are ForwardEuler, SymplecticEuler, RungeKutta4, Trapezoidal, and Constrained Backward Euler[4]. The latter is used for models in Chapters 1, 5, and 6.

\section{Friction and damping}

Artisynth enables the use of different damping techniques. In FE models these are related to rayleigh damping parameters [5]. The damping force $\left(\boldsymbol{f}_{d}\right)$ is related to two proportional damping terms:

$$
\boldsymbol{f}_{d}=\left(d_{M} \boldsymbol{M}+d_{K} \boldsymbol{K}\right) \boldsymbol{v}
$$

Where $d_{M}$ is the proportional damping associated with the motion of the FEM nodes and with the system's stiffness term. $\mathbf{M}$ is the lumped mass matrix and $\mathbf{K}$ the FE stifness matrix and $\mathbf{v}$ node velocity [4].

\section{Inverse modeling}

In forward modeling, the effect of muscle activation on the motion of the tongue is simulated. In Inverse modeling, the muscle activations needed for a given movement of a selection of nodes are calculated. The classical inverse approach used in the chapters of this thesis is described in Stavness et al.[6] and is part of the Artisynth framework. Also, hybrid strategies exist and can be combined with EMG to predict volunteer-specific muscle activation patterns[7]. As input, the desired position (target) of a point on the FE model is used. Using quadratic programming the muscle combinations needed to move the FE model to the desired position are calculated. Because multiple combinations of muscles can cause the same effect, a cost-function is used to choose the combination with the lowest combined muscle excitations (the lowest cost). The generic term for the cost function based on the Dantzig's LCP pivoting algorithm[8] is:

$$
\underset{x \in \mathbb{R}^{n}}{\operatorname{argmin}}\left\{\frac{1}{2} x^{T} Q x-x^{T} L\right\}, A x \geq b, A_{e q} x=b_{e q}
$$

With Q a symmetric matrix of size $n * n, \mathrm{~L}$ the linear $\mathrm{n}$-dimensional Colum vector, $A, A_{e q}, b_{e q}, b$ the (in) equality constraints and $\mathrm{x}$ the muscle activations. 
ArtiSynth utilizes multiple cost functions for multiple purposes. Relevant is the L2 regularization which penalizes the L2-norm (squared Euclidean distance) of different aspects, i.e. the deviation of simulated positions and target positions of the selected nodes, a term that punishes for the deviation of intended velocities of these nodes, a term that punishes for large or forbidden input signals and a term that damps the input signals. 


\section{REFERENCES}

1. Van Alphen MJA, Kreeft AM, Van Der Heijden F, Smeele LE, Balm AJM. Towards virtual surgery in oral cancer to predict postoperative oral functions preoperatively. Br J Oral Maxillofac Surg. British Association of Oral and Maxillofacial Surgeons; 2013;51: 747751. doi:10.1016/j.bjoms.2013.06.012

2. Nikishkov GP. Introduction to the finite element method [Internet]. Aizu-Wakamatsu; 2004. Available: https://homepages.cae.wisc.edu/ suresh/ME964Website/M964Notes/ Notes/introfem.pdf

3. Bonet J, Wood RD. Nonlinear continuum mechanics for finite element analysis, 2nd edition. Nonlinear Continuum Mechanics for Finite Element Analysis, 2nd Edition. 2008. doi:10.1017/CBO9780511755446

4. Lloyd JE, Stavness I, Fels S. ArtiSynth: A Fast Interactive Biomechanical Modeling Toolkit Combining Multibody and Finite Element Simulation. Studies in Mechanobiology, Tissue Engineering and Biomaterials. 2012. pp. 355-394. doi:10.1007/8415_2012_126

5. Lloyd JE. ArtiSynth Reference Manual [Internet]. 2014 [cited 5 Jun 2018]. Available: https://www.artisynth.org/Documentation/ArtisynthRefManual

6. Stavness IK. Byte Your Tongue: A Computational Model of Human Mandibular-Lingual Biomechanics for Biomedical Applications. Univercity of Britisch Columbia. 2010.

7. Eskes M. Surface electromyography in personalised modelling of the head and neck. University of Twente. 2017. doi:10.3990/1.9789036544474

8. Cottle RW, Dantzig GB. Complementary pivot theory of mathematical programming. Linear Algebra Appl. 1968; doi:10.1016/0024-3795(68)90052-9 


\section{APPENDIX B}

To avoid manual manipulation of the tracks in the atlas model, four filtering steps were used. A filter was only applied if it contributed to removal of faulty tracks. The first filter checked for deviations in the tracts from a global angle. This only worked well for muscle bundles with one global direction. The next filter calculated the angle within a single track. This did not work well with curved muscles like the superior longitudinal muscle. The third filter checked for the number of neighbors and at a certain distance, thus removing tracks that are too far from the rest. The alpha shape controlled the curvature of the convex hull that enclosed a muscle bundle and is explained in the Matlab documentation (https://nl.mathworks.com/ help/matlab/ref/alphashape.html).

Table B.1 - overview of different filters used to manipulate tracks in the model.

\begin{tabular}{|c|c|c|c|c|}
\hline & $\begin{array}{l}\text { The angle of the } \\
\text { vector cannot } \\
\text { deviate more } \\
\text { than ... }{ }^{0} \text { from the } \\
\text { total mean vector } \\
\text { direction of all } \\
\text { tracks. }\end{array}$ & $\begin{array}{l}\text { The angle of the } \\
\text { vector cannot } \\
\text { deviate more } \\
\text { than ... }{ }^{0} \text { from the } \\
\text { total mean vector } \\
\text { direction of one } \\
\text { muscle track. }\end{array}$ & $\begin{array}{l}\text { Tracks with less } \\
\text { than ... neighbors } \\
\text { at a distance of } \\
\text {... mm will be } \\
\text { removed }\end{array}$ & Alpha shape \\
\hline Vertical & 45 (z-axis) & 45 & $3 / 0.03$ & 0.011 \\
\hline Transverse & & & $5 / 0.20$ & 0.03 \\
\hline Superior longitudinal & & & $6 / 0.20$ & 0.014 \\
\hline Mylohyoid & & & $5 / 0.05$ & 0.015 \\
\hline Inferior longitudinal & 45 (y-axis) & 45 & $5 / 0.04$ & 0.011 \\
\hline hyoglossus & & 45 & $3 / 0.04$ & 0.011 \\
\hline Geniohyoid & & & $3 / 0.02$ & \\
\hline enioglossus & & 45 & $10 / 0.10$ & 0.011 \\
\hline Digastricus & & 45 & $3 / 0.03$ & 0.011 \\
\hline
\end{tabular}




\section{APPENDIXC}

Figure C. 1 shows a comparison between the generic model and the atlas model upon billateral muscle activation. The Generic model is based on the muscle anatomy from the Visible Human Project [1,2], and the atlas model (without the personalization step) is based on an average of CSD images from 10 healthy participants [3]. In the generic model, the genioglossus (GG) is divided into three individually controllable muscle parts, while GG in the atlas model is only divided into two $[3,4]$. The atlas model lacks the styloglossus (STY) and the generic model lacks the digastricus (DG) muscle due to different conceptualization steps [3]. Both use the Artisynth implementation of the threorie of Blemker et al. [5] for muscle activation and stiffening. The appearance of the models is different, as the atlas model has a blunt tongue tip and a more round shape overall. The different origins of the muscle bundles cause the models to behave differently upon activation. While the overall effect of the activation of each muscle bundle are comparable, it is especially the extent of movements that differ. 


\section{Generic Model}

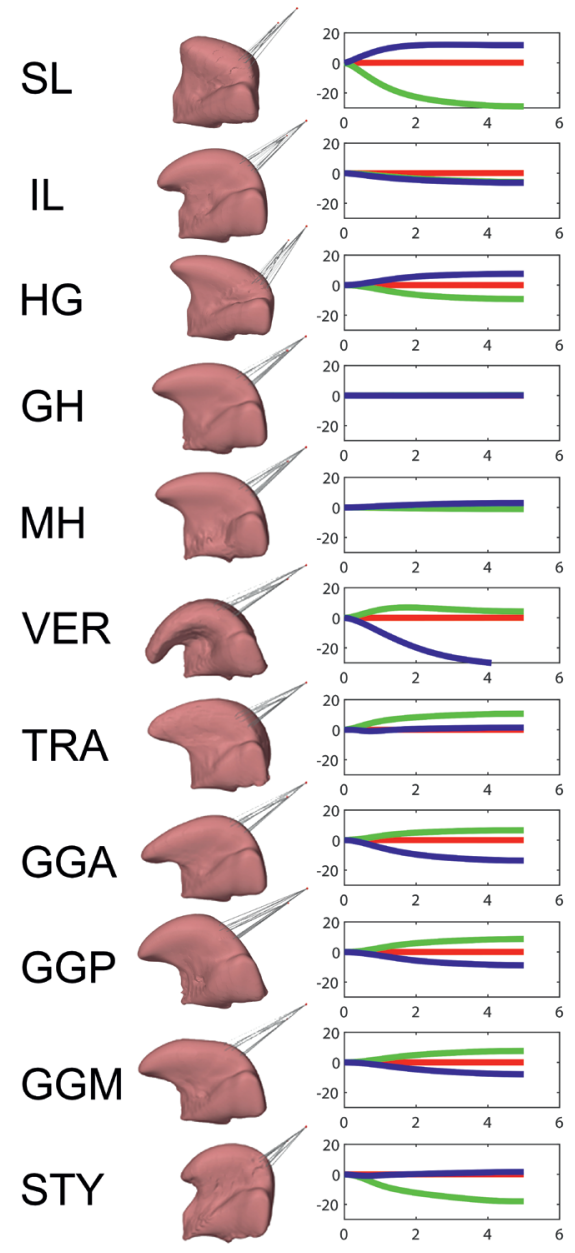

Atlas Model
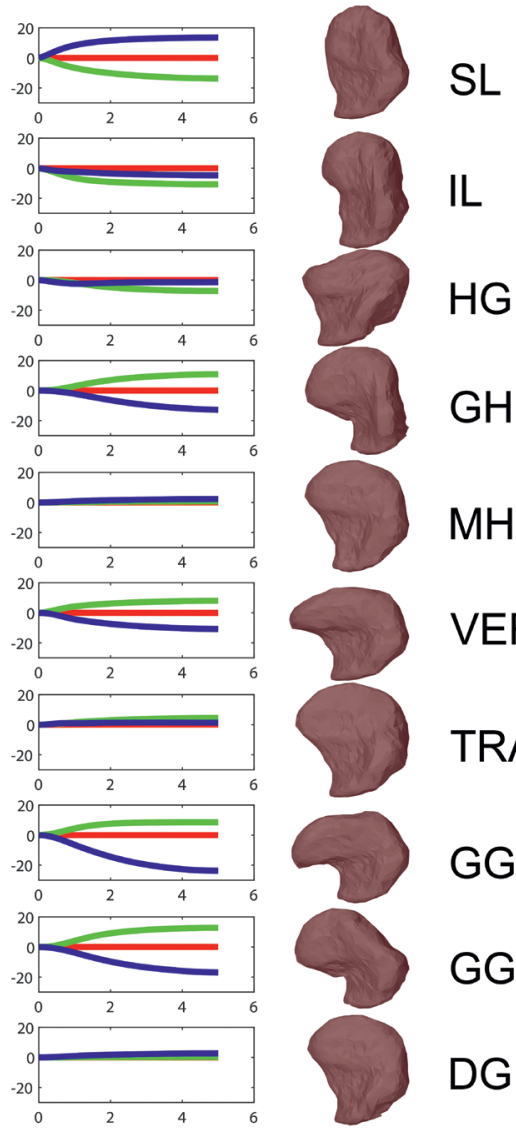

$\mathrm{GH}$

$\mathrm{MH}$

VER

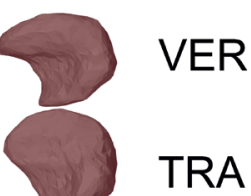

TRA

GGA

GGP

DG

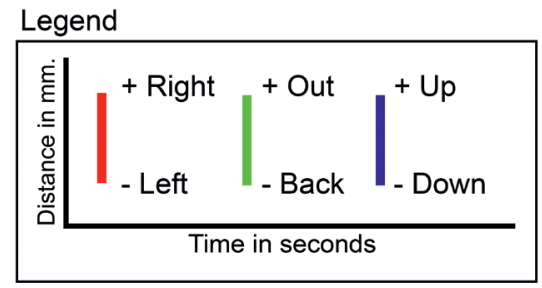

Figure C.1: A comparison between the tongue-tip position of the generic (left) model and the atlas model (right) upon billatateral muscle activation (30\% of maximum force). The red line is the + direction is the movement to the right and the - direction is the movement to the left. The green line in the + direction represents the outwards movement, - the direction of the backward movement. The Blue line + is the upward movement and - is the downward movement. The muscle abbreviations: genioglossus, -posterior (GGP), -middle (GGP), -anterior (GGA), geniohyoid muscle (GH), hyoglossus (HG), inferior longitudinal muscle (IL), mylohyoid muscle (MH), superior longitudinal muscle (SL), transverse muscle (TRA), vertical muscle (VER), styloglossus (STY) and digastricus (DG) 


\section{REFERENCES}

1. Buchaillard S, Perrier P, Payan Y. A biomechanical model of cardinal vowel production: muscle activations and the impact of gravity on tongue positioning. J Acoust Soc Am. 2009;126: 2033-2051. doi:10.1121/1.3204306

2. NIH. NLM's Current Bibliographies in Medicine, Visible Human Project ${ }^{\circledR}$ [Internet]. 2007 [cited 3 Jul 2018]. Available: http://www.nlm.nih.gov/archive/20120907/pubs/ cbm/visible_human_2007.html

3. Kappert KDR, Voskuilen L, Smeele LE, Balm AJM, Jasperse B, Nederveen AJ, et al. Personalizedbiomechanicaltonguemodelsbasedondiffusion-weightedMRIandvalidated using optical tracking of range of motion. Manuscript submitted for publication. 2020;

4. Kappert KDR, van Alphen MJA, van Dijk S, Smeele LE, Balm AJM, van der Heijden F. An interactive surgical simulation tool to assess the consequences of a partial glossectomy on a biomechanical model of the tongue. Comput Methods Biomech Biomed Engin. 2019;22: 827-839. doi:10.1080/10255842.2019.1599362

5. Blemker SS, Pinsky PM, Delp SL. A 3D model of muscle reveals the causes of nonuniform strains in the biceps brachii. J Biomech. 2005;38: 657-665. doi:10.1016/j. jbiomech.2004.04.009 


\section{ACKNOWLEDGEMENTS / DANKWOORD}

Dit proefschrift draag ik op aan mijn vader. Die zonder dat hij er de afgelopen 5 jaar bij kon zijn, mij altijd heeft gesteund, en zonder het te weten misschien nog wel het meest heeft bijgedragen van iedereen.

Om te beginnen wil ik mijn promotor prof. dr. A.J.M. Balm, de geestelijk vader van het Virtual Therapy project, bedanken. Zijn doorzettingsvermogen en onuitputtelijk optimisme wisten mij op de momenten waarop alles wat minder helder was altijd weer van nieuwe energie te voorzien, zelfs vanuit zijn vakantieadres. Naast chirurg, professor en promotor is hij namelijk een geweldige mentor en een heel fijn mens.

Dr. ir. F. van der Heijden, promotor en de tweede vader van het Virtual Therapy project om het zo maar te zeggen. Wij gaan nog verder terug, want al in 2014 vroeg jij of ik het interessant vond om me voor een stage te verdiepen in het programma genaamd "ArtiSynth". We kunnen allemaal deze thesis lezen en zien wat daarvan gekomen is. Jij wist altijd net op momenten dat Fons en ik even vast zaten de zaken van een hele andere kant te belichten, zodat we weer vorderingen konden maken. Een onmisbare factor in de afgelopen 5 jaar!

Prof. dr. ir. C.H. Slump, promotor, was met name betrokken bij het opzetten van het promotietraject en stelde als buitenstaander welkome kritische vragen. Ook kon hij met een uiterst serieus gezicht voorafgaand aan elke jaarlijkse meeting zeggen dat dit moment wel eens het einde van mijn promotietraject zou kunnen betekenen. Samen met prof. dr. T.J.M. Ruers, prof. dr. U.A van der Heide, dr. J. Nijkamp, prof. dr. L.E Smeele en de andere promotoren zat hij ook in de supervisory committee vanuit de Onderzoekschool Oncologie Amsterdam (OOA) om de voortgang van het promotietraject te bewaken. Bedankt voor alle nuttige feedback.

Prof. dr. L.E Smeele en verder ook dr. R.J.J.H. van Son wil ik bedanken voor de goede feedback op stukken, de initiatieven voor het Virtual Therapy project en de fijne sparmomenten op de maandagen. Daarnaast wil ik ook de chirurgen, secretaresses en staf van de afdeling hoofd-halsoncologie en -chirurgie bedanken voor de ontzettend leuke en inspirerende tijd bij het AvL. Evenals alle promovendi en collega's op de tweede en derde etage van het O-gebouw.

Prof. dr. J.N. Kok, prof. dr. ir. H.F.J.M. Koopman, prof. dr. ir. S. Stramigioli, prof. dr. M.W.M. van den Brekel en prof. dr. Y. Payan wil ik bedanken voor het vrijmaken van hun waardevolle tijd en voor het plaatsnemen in de promotiecommissie en het beoordelen van dit proefschrift. 
Prof. S. Fels and dr. J.E. Lloyd, thank you for the opportunity to learn about biomechanical modelling and Artisynth at the University of British Columbia, and for the online support with all my questions during my $\mathrm{PhD}$. Carly Ottenbreit, thank you for the language support and editing of several chapters from this thesis. Your valuable contributions have taken this thesis to the next level.

Nog steeds starten veel technische geneeskunde studenten onder de vleugels van dr. M.J.A. van Alphen, tevens special expert binnen de promotiecommissie. Bij Maarten kon ik altijd terecht met mijn verhaal en voor advies. Je kan hem alles vragen, heeft overal een antwoord op en is altijd gezellig. Het hoogtepunt was vaak de woensdagmiddag waarop de jolige Maarten zin had in een bakkie en de vragen des levens kwam bespreken. Bedankt Maarten voor alle mooie momenten!

Anderhalf uur lang met 32 sensoren op je hoofd de meest rare gezichtsuitdrukkingen maken, is het eerste wat ik mij kan herinneren van mijn stages op de afdeling bij dr. M. Eskes. Hoewel we ons op andere aspecten van het onderzoek focusten konden we elkaar altijd vinden bij onze struggles met ArtiSynth. Ook kon ik altijd terecht bij jou voor advies. Daarnaast kon ik natuurlijk genieten van al onze koffie- en dumpertmomenten.

Simone van Dijk, het brein achter de eerste Range of Motion-metingen (samen met Maarten). Bedankt voor de (helaas niet al te lange) tijd in het AvL, maar ook voor jouw tijd daarna waarin we samen aan maar liefst drie papers hebben kunnen werken.

Luuk Voskuilen, mede-auteur en parttime kamergenoot (hij zat liever in het AMC), wist mij altijd scherp te houden met zijn kritische blik en zijn brein als een encyclopedie. Naast veel intelligente conversaties hebben we altijd lol kunnen maken met de vele wisselende kamergenoten door de jaren heen: Rebecca Louhanepessy bedankt voor alle diepgaande en filosofische gesprekken en al het plezier dat we hebben beleefd in de afgelopen jaren! Maartje, bedankt voor alle lol en soms absurde grappen/gesprekken, maar bovenal de lijst met podcasts waar ik nooit meer vanaf kom (No Such Thing as a Fish is echt een aanrader)! Of het nou de nerd-grappen waren, of het in elkaar zetten van (incomplete) IKEAspullen, Bence Halpern en ik vermaakten ons altijd. Maar daarnaast heeft Bence mij ook goed kunnen helpen met zijn kennis van statistiek en deep learning. Bence, Egészségére!

Daarnaast wil ik 3 studenten bedanken met een dubbelfunctie als kamergenoot. Kicky van Leeuwen, Sander Boonstra en Eline van Staveren: bedankt voor de leuke tijd op onze kamer, maar ook voor jullie hulp bij het klinisch onderzoek. Daarnaast wil ik ook alle andere studenten bedanken die mee hebben geholpen 
bij het klinische onderzoek of inhoudelijk werk: Mirthe van Erp, Freeke Porte, Jouke Ubbink, Aline Kronenberg, Tim Boers, Fleur de Geer, Timon ter Braak, Sophie Ligtenstein en Sarah Verboom.

Un autre nom que je ne devrais vraiment pas oublier est Nathanael Connesson. Lui et moi avons discuté des problèmes liés à la mesure de l'élasticité de la langue. Sur une période de dix-huit mois, nous étions occupés aux moments les plus divers à rechercher des solutions aux analyses les plus impossibles. Il est remarquable de voir combien de temps Nathanael a consacré à ce projet pour rendre possible la publication de ce manuscrit. Merci beaucoup!

Bedankt dispuut MSCS voor alle lol in goede tijden en steun in lastige tijden. Ik vind het geweldig dat we zelfs na onze studententijd nog zoveel aan elkaar hebben en ik hoop dat we ondanks de coronaperikelen er op 18 december nog een groot feest van kunnen maken.

Bedankt (oud-)bestuursleden van de NVvTG voor een mooie en inspirerende tijd waar we samen werkten aan de ontwikkeling van de technisch geneeskundige. Maar ook voor de fijne afwisseling van dit werk naast mijn promotie. De komende tijd gaan we nog veel belangrijke stappen met elkaar zetten!

Martijn en Colin, two brothers from another mother die je altijd met de neus op de feiten drukken en zorgen dat zelfs een bijna-gepromoveerde wetenschapper zich nog gewoon als een kind kan gedragen. Maar ook zij moeten op 18 december in hun rol als paranimf laten zien dat ze zich als echte wetenschappers kunnen gedragen. Dat wat ze inhoudelijk niet hebben meegedragen maken ze goed met de hoeveelheid plezier die ik al vanaf het begin van de middelbare school met ze heb beleefd!

Naast al deze vrienden en collega's kan mijn familie natuurlijk niet ontbreken in dit dankwoord. Om te beginnen met mijn moeder: Liefste mam, waar pap een plekje hoog aan de pagina heeft, verdien jij een plek aan het einde (de laatste auteur in een artikel is vaak erg belangrijk). Deze thesis is een direct gevolg van jouw onvoorwaardelijke steun en, heel praktisch gezien, met name jouw inzet in de avonduren in mijn vroege levensjaren om mijn schrijf- en leesniveau te verhogen tot iets acceptabels. Ik ben zo ontzettend dankbaar voor de steun van jou en pap die ik in de afgelopen jaren heb gehad. Betere ouders dan jullie kan ik mij niet wensen! 
De band tussen broer en zus is vaak iets vanzelfsprekends. Daarom vind ik het des te meer bijzonder dat jij, Cleo, niet alleen mijn zus maar ook een echte vriendin van me bent. Hoewel we erg verschillend zijn, hebben we aan één woord genoeg om elkaar te kunnen begrijpen en sta je altijd voor me klaar.

Daarnaast zijn trotse opa's en oma's goud waard. Het geeft zoveel energie om te weten dat wat ik doe jullie trots maakt. Dank voor alle steun, liefde en een luisterend oor als ik met jullie praat over mijn 'PDDD'. Tanja en Johan, dank voor jullie steun interesse en natuurlijk voor de slaapplek en het heerlijke avondeten tijdens mijn stages.

Lieve Eveline, lief dropje, je bent voor mij verhuisd naar de Randstad om samen dit avontuur aan te gaan. Promoveren gaat gepaard met ups en downs en jij was altijd de eerste om met mij de ups te vieren en de downs te relativeren. Je bent altijd betrokken bij wat ik doe, denkt overal bij mee en je bent degene die mij echt door en door begrijpt. Dit was echter ons begin, en ik weet zeker dat wij samen nog lang gaan genieten van wat nog komen gaat. 


\section{ABOUT THE AUTHOR}

Kilian Kappert was born on July $12^{\text {th }} 1991$ in Hengelo, the Netherlands. In 2009 he finished secondary school at "Het Bataafs Lyceum" in Hengelo. The same year he moved to Enschede to study Technical Medicine. After a year of being on the board of the study association Paradoks he went on with the technical medicine master track "Medical Imaging \& Intervention". After internships at the Amsterdam University Medical Center, Ziekenhuisgroep Twente and Medisch Spectrum Twente he wrote is graduation thesis under supervision of prof dr. A.J.M. Balm and dr. ir. F. van der Heijden at the Netherlands Cancer Institute Antoni van Leeuwenhoek hospital. A part of this graduation internship took place at the University of British Columbia under supervision of prof dr. Fels to learn more about biomechanical modeling. This internship led to the start of Kilian's $\mathrm{PhD}$ at the Netherlands Cancer Institute and University of Twente, resulting in the thesis that is now in front of you. During his promotion he cofounded Wrable B.V., a fitness wearable company, and was member of the board at the Dutch Association for Technical Medicine (NVvTG). After his PhD he started as coordinator for complex diabetes research at Ziekenhuisgroep Twente. 
